\title{
Novel MOS-Based Biomolecule Detection Techniques
}

by

\section{Ryan Hunter Griffin, B.Eng., M.A.Sc.}

A thesis submitted to the Faculty of Graduate and Postdoctoral Affairs in partial fulfilment of the requirements for the degree of

\section{Doctor of Philosophy}

in

Electrical and Computer Engineering

Ottawa Carleton Institute for

Electrical and Computer Engineering

Department of Electronics

Carleton University

Ottawa, Ontario, Canada

December 2014

Copyright (c) 2014

Ryan Hunter Griffin 


\section{Abstract}

This thesis advances the state of the art in biomolecule detection by allowing for quantitative detection using radioactive labels whose decay is detected by a pixelated complementary metal-oxide-semiconductor (CMOS), or CMOS-compatible, sensor. Additionally, the usefulness of this technology as a testbed for radioimmunotherapy (RIT) pharmaceuticals is considered.

For the first time a CMOS image sensor has been used to detect the presence of radiolabelled target biomolecules captured on a functionalized surface. Using aptamer functionalization the system successfully detected phosphorus-32 labelled adenosine triphosphate (ATP) at a surface concentration of $2.3 \times 10^{7}$ molecules $/ \mathrm{cm}^{2}$, well below those typically associated with fluorescence-based sensor architectures. The system has also demonstrated its amenability to multiplexed biomolecule detection. Geant4, a Monte Carlo toolkit for simulating the passage of radiation through matter, was used to model the detection system. This system has applications in quantitative biomolecule detection and in the development of RIT pharmaceuticals employing beta particle emitting isotopes.

Also for the first time, a MOS sensor has been designed, fabricated, and tested for use in the characterization of targeted alpha therapy (TAT) pharmaceuticals. The sensor consists of a $16 \times 16$ array of $100 \mu \mathrm{m}$ square alpha particle sensitive cells 
fabricated in-house using a simple nMOS process. A subset of the cells are functionalized for the attachment of chelators under investigation for new pharmaceuticals. To demonstrate the utility of this sensor as a characterization platform, cells functionalized with 1,4,7,10-tetraazacyclododecane-1,4,7,10-tetraacetic acid (DOTA)DNA conjugates were used to chelate americium-241 from solution, and the alpha particle emissions over the surface of the IC measured. The IC was able to quantitatively determine the amount of alpha emitter present over each cell, allowing the chelator and chelating chemistry to be assessed. Without any optimization of the chelation chemistry, a $21 \%$ increase of emissions was detected on cells functionalized with DOTA relative to unfunctionalized cells. 


\section{Acknowledgments}

I am grateful to many people who have encouraged and supported me as I took this opportunity to explore my curiosity. My supervisor, mentor, and friend, Dr. Garry Tarr has been supporting me, patiently, from the very beginnings of my academic career. I cannot thank him enough for all that he has done.

It was by taking the opportunity to continue my education that I met my wife, Svetlana Demtchenko. She has had a tremendous impact on my life, and has been a constant source of encouragement. She's a wonderfully bright, honest and caring woman who has truly made this experience life changing. Thank you as well to Dmitri and Galina Demtchenko for welcoming me into their family with open arms.

Of course I would like to acknowledge the collaborators who made this work possible: Dr. Maria DeRosa, Dr. Gregory Lopinski, Olga Mozenson, Michael Beking, and Erin McConnell. This work would not have been possible without their functionalization chemistries, aptamer synthesis, and in some cases, steady hands while conducting experiments.

Dr. Steven McGarry, although not officially my supervisor, your open door, guidance, endless fountain of knowledge, overall spirit and sense of humour has been very much appreciated.

A sincere thank you to all the Carleton University Microfabrication Lab staff, Carol Adams, Rob Vandusen, Rick Adams, and Angela Mccormick. You were all invaluable in processing my devices, and providing me the necessary substrates for 
my experiments.

Chipworks - Dick James in particular - are thanked for providing information regarding the Canon 705S image sensor.

The Department of Electronics staff, Blazenka Power, Anna Lee, and Sylvie Beekmans have done, and continue to do, great work making the department run as best it can. Thank you to Scott Bruce for providing the computing resources I needed to complete my simulation work, and thank you Nagui Mikhail for providing hardware and lab space as needed.

For the open, random, and occasionally insightful discussions had over lunch, I'd like to thank Dr. Pietro Chyurlia, Dr. Daniel Burke, Che Knisely, Nathan Jess, and Thomas Peppler.

To my parents, Joanne and Donald Griffin, thank you for your endless patience and support. It has been a long, sometimes difficult road, but none of this would have been possible without you.

Lastly, thank you to my friends who provided distraction and listened to me vent as necessary. In particular, thank you Matthew Witkowicz, Dylan Hennessey, Christopher Mack, Alasdair Rankin, Dr. Antony Robert, Clement Tse and Dr. Lindsay Beaton. 


\section{Table of Contents}

Abstract $\quad$ ii

Acknowledgments $\quad$ iv

Table of Contents vi vi v

List of Tables $\quad$ X

List of Figures $\quad$ xi

List of Acronyms $\quad$ Xv

List of Symbols $\quad$ xx

1 Introduction 1

1.1 Motivation . . . . . . . . . . . . . . . . . . 1

1.2 Objectives . . . . . . . . . . . . . . . . . . . . 3

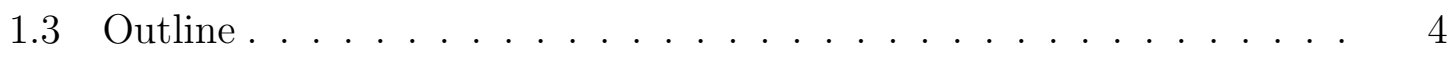

2 Review 5

2.1 Radiation .......................... 5

2.1.1 Radiation Types .................. 6

2.1.2 Interaction of radiation with matter . . . . . . . . . . 7

2.1.3 Biological effects of ionizing radiation . . . . . . . . . . . 17 
2.1.4 Simulation tools . . . . . . . . . . . . . . . . 18

2.2 Radiation Detectors . . . . . . . . . . . . . . . 20

2.2.1 Semiconductor Basics and the PN Junction Diode . . . . . . . 20

2.2.2 Silicon Radiation Detectors . . . . . . . . . . . . . . . 23

2.2.3 Noise Sources in Silicon Detectors . . . . . . . . . . . . . . . . 29

2.2.4 Limitations on Spatial Resolution in Silicon Detectors . . . . . 32

2.2.5 Example Silicon Sensors . . . . . . . . . . . . . . . . 32

2.3 Radioimmunotherapy . . . . . . . . . . . . . . . . . 35

2.3.1 Targeting Agents and Methods . . . . . . . . . . . 36

2.3.2 Radioisotope Payloads . . . . . . . . . . . . . . . 36

2.3.3 Current State of Pharmaceutical Development . . . . . . . . . 39

2.4 Chemistry . . . . . . . . . . . . . . . . . . 40

2.4.1 Gold-Thiol Chemistry . . . . . . . . . . . . . . 40

2.4.2 Silane Chemistry . . . . . . . . . . . . . . . 41

2.5 Similar Work . . . . . . . . . . . . . . . . . 41

3 Simulation $\quad 43$

3.1 Geant4 Introduction . . . . . . . . . . . . . . . . . . 43

3.2 Geant4 Simulations . . . . . . . . . . . . . . . . 46

3.2.1 Software Validation . . . . . . . . . . . . . . . 46

3.2.2 Charge \& Energy Deposition . . . . . . . . . . . . . . . 51

3.2.3 Packaging and Backscattering Effects . . . . . . . . . . . . 55

3.3 Sentaurus Introduction . . . . . . . . . . . . . . . . . . . 62

3.4 Sentaurus Simulations . . . . . . . . . . . . . 63

3.5 Conclusion . . . . . . . . . . . . . . . . . . 70

4 Quantitative detection of a $\beta^{-}$emitting isotope on a functionalized $\begin{array}{ll}\text { substrate using a CMOS sensor } & 71\end{array}$ 
$4.1 \quad$ Experimental . . . . . . . . . . . . . . . . . . . . 72

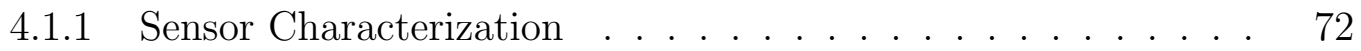

$4.1 .2 \quad$ Experimental Procedure . . . . . . . . . . . . . . . . . . 74

4.2 Experimental Results . . . . . . . . . . . . . . . . . . 78

4.2 .1 Quantum Efficiency . . . . . . . . . . . . 78

4.2.2 Radiolabelled Measurements . . . . . . . . . . . . . . . 78

4.2 .3 Background Calculation . . . . . . . . . . . . . . 79

4.2.4 Surface concentration of bound ATP . . . . . . . . . . 81

4.3 Discussion . . . . . . . . . . . . . . . . . . . . . . . . . . . . 82

4.4 QE Simulation . . . . . . . . . . . . . . . 84

4.5 Conclusion . . . . . . . . . . . . . . . . . . . . 85

5 MOS testbed for the characterization of targeted $\alpha$ therapy phar$\begin{array}{ll}\text { maceuticals } & 87\end{array}$

$5.1 \quad$ Experimental . . . . . . . . . . . . . . . . . . . . . . . . . . 89

5.1.1 Sensor Design and Preparation _. . . . . . . . . . . 89

5.1 .2 Chemistry . . . . . . . . . . . . . . . . 91

$5.1 .3 \quad$ Experimental Protocol . . . . . . . . . . . . . . . . . 94

5.2 Results and Discussion . . . . . . . . . . . . . . . . 96

5.2 .1 Background Count Rate . . . . . . . . . . . . . . 96

5.2 .2 Americium Chelation Experiment . . . . . . . . . . . . 96

5.2 .3 Geant4 Modelling . . . . . . . . . . . . . . . . . . . . 100

5.2 .4 Continued Development . . . . . . . . . . . . . . 102

5.3 Conclusion . . . . . . . . . . . . . . . . . . . . . . . 103

6 Conclusions 104

6.1 Future Work . . . . . . . . . . . . . . . . . . . 105

6.2 Ultimate Detection Limit . . . . . . . . . . . . . . . . 106 
6.3 Disseminations . . . . . . . . . . . . . . . . . . . . . . . . . . 109

$\begin{array}{ll}\text { List of References } & 110\end{array}$

$\begin{array}{ll}\text { Appendix A Sentaurus Input Files } & 123\end{array}$

A.1 2D Substrate Diode Structure . . . . . . . . . . . . . . . . 123

A.2 2D Substrate Diode Device Simulation _ . . . . . . . . 126

A.3 2D 705 S Structure $\ldots \ldots \ldots \ldots \ldots \ldots \ldots \ldots \ldots$

A.4 2D 705S Device Simulation . . . . . . . . . . . . . 133

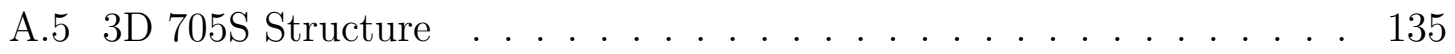

A.6 3D 705S Device Simulation . . . . . . . . . . . . . . 142

$\begin{array}{ll}\text { Appendix B Raw Chelation Data } & 145\end{array}$

B.1 Data from Figure $5.4(\mathrm{c}) \ldots \ldots \ldots \ldots \ldots$ 


\section{List of Tables}

2.1 Characteristic distance of charged and uncharged radiation. . . . . . . 8

2.2 Non-exhaustive list of isotopes of interest for RIT pharmaceuticals.

$\left({ }^{*}\right)$ indicates a weighted average. . . . . . . . . . . . . . . . 37

2.3 Summary of the properties of $\alpha$ and $\beta^{-}$emitting radioisotopes for RIT. 38

3.1 Summary of Geant4 ${ }^{32} \mathrm{P}$ simulation results. . . . . . . . . . . 53

3.2 Summary of GEANT4 ${ }^{90} \mathrm{Sr}$ simulation results. . . . . . . . . . . . 55

3.3 System level GEANT4 simulation results of the Canon 705S sensor and packaging for both ${ }^{32} \mathrm{P}$ and ${ }^{90} \mathrm{Sr}$ simulated with $50 \times 10^{6}$ events. Note that the glass coverslip was not present in the simulation with ${ }^{90} \mathrm{Sr}$. $\quad 61$

3.4 Canon 705S process details. (*) denotes information from Chipworks. 63

3.5 Canon 705S sensor specifications . . . . . . . . . . . . . . 64 


\section{List of Figures}

2.1 Bragg curve for a $5.49 \mathrm{MeV} \alpha$ particle in air. . . . . . . . . . . . . . 9

2.2 Simulation of one hundred $6 \mathrm{MeV} \alpha$ particles in silicon using SRIM2008. 10

2.3 Geant4 simulation of three normally incident $1 \mathrm{MeV} \beta$ particles passing through a silicon slab starting at the origin. Each point represents the final position of an ionized electron. The YZ plane forms the slab's surface, while the X axis is depth. . . . . . . . . . . . . . . . 12

2.4 General form of the Landau distribution. . . . . . . . . . . . . . . 13

2.5 Compton scattering. . . . . . . . . . . . . . . 16

2.6 Silicon $\mathrm{PN}$ junction. . . . . . . . . . . . . . . . . . . 22

2.7 CCD cross section. . . . . . . . . . . . . 26

2.8 CCD operation. . . . . . . . . . . . . . . 26

2.9 Passive and active pixel sensor pixel schematics. . . . . . . . . . . 27

2.10 CMOS MAPS readout architecture. . . . . . . . . . . . 28

2.11 Back-thinned detector. . . . . . . . . . . . . . . . . 33

2.12 Actinium-225 decay chain. . . . . . . . . . . . . . . 39

3.1 Geant4 simulation flow. . . . . . . . . . . . . . . . 45

3.2 Geant4 software validation structure showing three $5 \mathrm{MeV} \beta^{-}$particles. 47

3.3 Histogram of charged secondaries generated by twenty thousand $5 \mathrm{MeV}$ $\beta^{-}$particles passing through a $6.4 \mu \mathrm{m}$ square and $3 \mu \mathrm{m}$ deep block of silicon using the GeAnt4 MicroElec models. . . . . . . . . . . . . . . 48 
3.4 Histogram showing the track length of charged secondaries generated by twenty thousand $5 \mathrm{MeV} \beta^{-}$particles in a block of silicon using the Geant4 MicroElec models. . . . . . . . . . . . . . . . . . . 48

3.5 Data from the NIST ESTAR database showing the stopping power of $\beta^{-}$particles in silicon as a function of energy. . . . . . . . . . . . . . 49

3.6 Histogram showing the energy deposited per unit length by twenty thousand $5 \mathrm{MeV} \beta^{-}$particles in a block of silicon using the GEANT4 MicroElec models. . . . . . . . . . . . . . . . . . . . . 50

3.7 Geant4 simulation of fifty thousand $5 \mathrm{MeV} \beta^{-}$particles normally incident on a $2 \mathrm{~cm}$ cubed block of silicon using the Livermore models.

3.8 Geant4 simulation of two hundred thousand $5.4 \mathrm{MeV} \alpha$ particles normally incident on a block of silicon using the standard models. . . . . 52

$3.9{ }^{32} \mathrm{P}$ and ${ }^{90} \mathrm{Sr} \beta^{-}$emission spectrum. . . . . . . . . . . . . .

3.10 GeAnT4 charge and energy deposition simulation results for ${ }^{32} \mathrm{P}$ using the MicroElec model with a $16.7 \mathrm{eV}$ cutoff, as well as the Livermore model with $250 \mathrm{eV}$ and $10 \mathrm{eV}$ cut off for 50,000 normally incident $\beta^{-}$ particles. . . . . . . . . . . . . . . . .

3.11 GEANT4 charge and energy deposition simulation results for ${ }^{90} \mathrm{Sr}$ using the MicroElec model with a $16.7 \mathrm{eV}$ cutoff, as well as the Livermore model with $250 \mathrm{eV}$ and $10 \mathrm{eV}$ cut off for 50,000 normally incident $\beta^{-}$ particles. . . . . . . . . . . . . . . . 56

3.12 Canon 705S CMOS MAPS packaging. . . . . . . . . . . 58

3.13 Canon 705S CMOS MAPS simulation structure showing ten $900 \mathrm{keV}$ $\beta^{-}$particles emitted isotropically from a point source. A $5 \times 5 \mathrm{~mm}^{2}$ surface area is shown. . . . . . . . . . . . . . . . . 58 
3.14 2D histogram, and projections, of ${ }^{32} \mathrm{P} \beta^{-}$particles entering and exiting the surface of a Canon 705S image sensor with $50 \times 10^{6}$ events simulated using GeAnt4 with Livermore models. . . . . . . . . . . . 60

3.15 Stopping power in the Canon 705S CMOS MAPS packaging and sensor from $50 \times 10^{6}$ events from an isotropic ${ }^{32} \mathrm{P}$ point source. . . . . . . . 61

3.16 Baseline Sentaurus simulation structure and doping levels. . . . . . . 65

3.17 Sentaurus simulation of a $1 \mu \mathrm{m}$ junction diode for increasing range of the incident particle. . . . . . . . . . . . . . . . 66

3.18 Sentaurus structures and doping profile used to model the Canon 705S

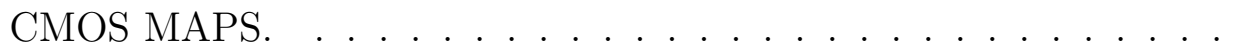

3.19 2D simulation results of the 705S MAPS showing the effects of strike offset and LET on charge collection for normally, and transversally, incident ionizing radiation. . . . . . . . . . . . . . . . .

4.1 Round $5 \mathrm{~mm}$ radius coverslip, 80-130 $\mu \mathrm{m}$ thick, spotted with one or two aptamer regions via a silane based chemistry. . . . . . . . . . . 75

4.2 Fluorescence measurements of aptamer functionalized surfaces at $1 \mathrm{mM}, 0.5 \mathrm{mM}$ and $0.1 \mathrm{mM}$ ATP concentrations. . . . . . . . . . 75

$4.3 \beta^{-}$labelled experiment procedure. . . . . . . . . . . . . 76

4.4 Experimental results showing the number of counts detected when exposing the 705S MAPS to an ${ }^{90} \mathrm{Sr}$ source for varying exposure times. .

4.5 Experimental results of ${ }^{32} \mathrm{P}$ labelled ATP selectively attached to an aptamer functionalized glass substrate and measured with a CMOS image sensor at room temperature. . . . . . . . . . . . . 80

4.6 QE measurement results with a single ${ }^{32} \mathrm{P}$ spot on a glass coverslip. . $\quad 85$

4.7 Raw QE measurement data with a single spot of dried ${ }^{32} \mathrm{P}$ labelled ATP on a glass coverslip. . . . . . . . . . . . . . . 
5.1 Images of a fully processed and packaged $\alpha$ RAM. Cells are indexed from top left $(1,1)$ to bottom right $(16,16)$. Wirebonds on rows 7 and 16 were broken accidentally during handling. Aside from the missing rows, all cells are functional. . . . . . . . . . . . . . . . . . . . . . 90

5.2 Self-spotting verification with thiol modified single-stranded DNA and its Cy3 labelled complement on $100 \mu \mathrm{m}$ square gold patterned cells.

5.3 Pictorial representation of experimental procedure for chelating americium from solution. . . . . . . . . . . . . . . . . . . 95

5.4 Experimental results after chelating ${ }^{241} \mathrm{Am}$ from solution. . . . . . . . 97

5.5 GEAnt4 simulation of $\alpha$ particle emissions on the $\alpha$ RAM surface. . . 101 


\section{List of Acronyms}

\begin{tabular}{ll} 
Acronym & Definition \\
\hline \hline ADC & Analog to Digital Converter \\
ADU & Analog to Digital Unit \\
AMS & Austria Microsystems \\
APS & Active Pixel Sensor \\
AR & Autoradiography \\
ASIC & Application Specific Integrated Circuit \\
ATP & Adenosine Triphosphate \\
BPSG & Borophosphosilicate Glass \\
CASINO & monte CArlo SImulation of electroN trajectory in sOlids \\
CCD & Charge Coupled Device \\
CDS & Correlated Double Sampling \\
CMOS & Complementary Metal Oxide Semiconductor \\
CSDA & Continuously Slowing Down Approximation \\
\hline
\end{tabular}




\begin{tabular}{|c|c|}
\hline $\mathrm{DC}$ & DNA Chelator \\
\hline DNA & Deoxyribonucleic Acid \\
\hline DSB & Double Strand Break \\
\hline dsDNA & Double Stranded Deoxyribonucleic Acid \\
\hline DSSSD & Double Sided Silicon Strip Detector \\
\hline DOTA & 1,4,7,10-Tetraazacyclododecane-1,4,7,10-Tetraacetic Acid \\
\hline DSLR & Digital Single Lens Reflex \\
\hline DRAM & Dynamic Random Access Memory \\
\hline DT & DNA Tether \\
\hline DTPA & Diethylene Triamine Pentaacetic Acid \\
\hline DTT & Dithiothreitol \\
\hline EBRT & External Beam Radiation Therapy \\
\hline EGS & Electron Gamma Shower \\
\hline FPN & Fixed Pattern Noise \\
\hline FRET & Fluorescence Resonance Energy Transfer \\
\hline FWHM & Full Width Half Maximum \\
\hline GCD & Gate Controlled Diode \\
\hline GEANT & GEometry ANd Tracking \\
\hline GUI & Graphical User Interface \\
\hline
\end{tabular}




$\begin{array}{ll}\text { GTP } & \text { Guanosine Triphosphate } \\ \text { GPS } & \text { General Particle Source } \\ \text { HEP } & \text { High Energy Physics } \\ \text { HPLC } & \text { High-Performance Liquid Chromatography } \\ \text { IC } & \text { Integrated Circuit } \\ \text { IMS } & \text { Institute for Microstructural Sciences } \\ \text { IR } & \text { Infrared } \\ \text { LET } & \text { Linear Energy Transfer } \\ \text { LOTOX } & \text { Low Temperature Oxide } \\ \text { mAbs } & \text { Monoclonal Antibodies } \\ \text { MAPS } & \text { Monolithic Active Pixel Sensor } \\ \text { MCNP } & \text { Monte Carlo Neutron Photon } \\ \text { NHP } & \text { Nano-Particle } \\ \text { MOP } & \text { Micro Channel Plates } \\ \text { MPP } & \text { Metal Oxide Semiconductor } \\ \text { Megapixel }\end{array}$




$\begin{array}{ll}\text { NRC } & \text { National Research Council } \\ \text { PBS } & \text { Phosphate Buffered Saline } \\ \text { PDMS } & \text { Polydimethylsiloxane } \\ \text { PENELOPE } & \text { PENetration LOss of Positrons and Electrons } \\ \text { PGM } & \text { Portable Gray Map } \\ \text { QE } & \text { Quantum Efficiency } \\ \text { RGB } & \text { Red, Green, Blue } \\ \text { RIC } & \text { Radioimmunoconjugate } \\ \text { RIT } & \text { Radioimmunotherapy } \\ \text { RMS } & \text { Root Mean Square } \\ \text { ROI } & \text { Region of interest } \\ \text { rSAv } & \text { Recombinant Streptavidin } \\ \text { SIMS } & \text { Steacie Institute for Molecular Sciences } \\ \text { SiPM } & \text { Silicon Photomultiplier } \\ \text { SOI } & \text { Silicon on Insulator } \\ \text { SRIM } & \text { Stopping Range of Ions in Matter } \\ \text { Single Strand Break }\end{array}$


TFA

TRT
Thin Film on ASIC

Targeted Radionuclide Therapy 


\section{List of Symbols}

\section{Symbols Definition}

\begin{tabular}{ll}
\hline \hline$\alpha$ & Alpha particle \\
$\lambda_{Z}$ & Lifetime of isotope Z \\
$A$ & Mass number \\
$\beta^{+}, e^{+}$ & Beta particle (positron) \\
$\beta^{-}, e^{-}$ & Beta particle (electron) \\
$\beta$ & Ratio of $v / c$ \\
$\gamma$ & Photon (gamma ray) \\
$T$ & Temperature \\
$n$ & Mass attenuation coefficient \\
$N_{Z}$ & Number of nuclei Z \\
$\eta$ & Quantum efficiency \\
$\nu$ & Neutrino \\
& Time or mass thickness
\end{tabular}




$\begin{array}{ll}t_{\frac{1}{2}} & \text { Half-life } \\ { }_{Z}^{A} X_{N} & \text { Parent nucleus } \\ { }_{Z}^{A} Y_{N} & \text { Daughter nucleus } \\ Z & \text { Atomic number }\end{array}$




\section{Chapter 1}

\section{Introduction}

\subsection{Motivation}

Biomolecule detection is used extensively in medical and biological research and is a key technique advancing these fields. Developing and characterizing new test beds for biomolecule detection can facilitate advancement of next generation cancer-treating pharmaceuticals. Detection techniques can be coarsely broken down into two types: labelled and label-free. Labelled techniques require an identifying molecule, often of a fluorescent nature, be attached to the biomolecule of interest. This identifying label can then be detected with suitable post-processing. Label-free techniques attempt to identify the biomolecule without the need to attach an identifying molecule [1]. There has also been some research in self-labelling techniques where a selective affinity reaction occurs between a known pre-labelled biomolecule and an unknown sample such that the reaction between the two allows the biomolecules in the unknown sample to be identified [2].

Although other means of identifying molecules exist, fluorescent labels are most often used. Fluorescent labels have proven to be very effective at determining the

presence of a particular biomolecule but are plagued by quantification problems. Nano-Particle (NP) labels have recently come of interest due to a variety of detection 
methodologies as well as their potential for multi-platform detection. NP labels can be prepared to provide fluorescent, magnetic, serial/barcode and radioactive identifiers or a combination thereof [3-8]. Of these identifiers, radioactive labels provide a detection mechanism which best lends itself to quantitative analysis of biological samples [9]. Radiolabelled methods do not require an external excitation source nor an optical system for readout, are not subject to fluorescent resonance energy transfer (FRET), and are inherently quantitative.

Radioactive labels have found use in a variety of medical and biological research technologies. Some examples of application include, but are not limited to, the following $[10,11]$ :

- Tissue imaging (autoradiography)

- Pharmaceutical studies and drug development

- Genetic studies

- In vitro cell growth

- DNA sequencing and hybridization studies

- Biological assays

- Cancer treatment

These applications all benefit from quantifiable detection of the radioactive labels used.

Of particular interest is the recent development of cancer-fighting pharmaceuticals employing $\alpha$ emitting isotopes. Radioimmunotherapy (RIT) pharmaceuticals are a class of cancer treating medications which use the energy of radioactive decay to kill cancerous tissue. A number of RIT pharmaceuticals exist utilizing $\beta$ emitting isotopes, and one $\alpha$ emitting pharmaceutical has recently been approved. A more thorough discussion on RIT pharmaceuticals can be found in Section 2.3. 
By leveraging known techniques in radioactive labelling and detection, a platform can be developed that allows for cost effective quantitative measurements of biomolecules improving over fluorescence based techniques while at the same time developing a testbed for next generation RIT pharmaceuticals.

\subsection{Objectives}

Advancing the state of the art in biomolecule detection by allowing for quantitative biomolecule detection using radioactive labels whose decay is detected by a pixelated complementary metal-oxide-semiconductor (CMOS), or CMOS-compatible, sensor and demonstrating the usefulness of this technology as a testbed for RIT pharmaceuticals are the primary goals of this work.

By collaboration with Carleton University's Chemistry Department, as well as the National Research Council's Steacie Institute for Molecular Sciences, quantitative detection of $\beta$ particle labelled biomolecules was performed using a commercial CMOS image sensor. In these experiments adenosine triphosphate (ATP) labelled with phosphorus-32 was captured selectively on an aptamer-functionalized glass substrate and the emission detected with a commercial CMOS image sensor $[12,13]$. GEANT4, a complex Monte Carlo toolkit for the simulation of particle passage through matter, in conjunction with Sentaurus, a semiconductor device simulation tool, was used to simulate and analyze the device $[14,15]$.

Targeted Alpha Therapy (TAT) is a type of radioimmunotherapy where $\alpha$ particle emitting isotopes selectively bind to, and kill, cancerous tissue. Radioactive isotopes are frequently tethered to a cancer-targeting agent by means of a chelator. Assessing the ability of the chelator to retain the particular isotope used is an important task and one to which an arrayed $\alpha$ particle sensitive integrated circuit (IC) compatible with TAT testing protocols would be well suited. A MOS IC designed by the author, 
and fabricated at Carleton University, was used for this demonstration [16-18]. Work was also done on guiding the development of the next generation version of the $\alpha$ sensitive IC used in these measurements suitable for fabrication at a commercial foundry.

\subsection{Outline}

This dissertation will begin with a review of radiation, radiation detection, radiopharmaceuticals, and biomolecule attachment chemistries in Chapter 2. Chapter 3 will present details of the simulations used to characterize silicon based sensors for charged particle radiation for the experiments discussed here. Chapter 4 will present experimental results utilizing a CMOS image sensor to detect biomolecules labelled with a $\beta$ emitting isotope on a functionalized substrate. Chapter 5 will present experimental results of $\alpha$ particle emitting isotopes directly, and selectively, attached to the surface of a custom $\alpha$ particle sensitive IC. Chapter 6 concludes this thesis with comments on the results and suggestions for future work. 


\section{Chapter 2}

\section{Review}

An understanding of the nature of ionizing radiation, as well as its detection, is essential in developing a system for quantified detection of radiolabelled biomolecules. From understanding the nature of radiation, it becomes clear how it can be well served as both a label for detection as well as a tool for treating cancer. What follows is a review of the nature of radiation, radiation detection, radioimmunotherapy, as well as the background required for radiolabelled biomolecule experiments performed in this dissertation.

\subsection{Radiation}

Radioactive isotopes used in medical and biological research are emitters of $\alpha$ particles, high-energy electrons and positrons $\left(\beta^{-}\right.$and $\beta^{+}$particles respectively) and $\gamma$-rays. Each type of radiation has unique properties and various sensors exist for their detection. The detectors themselves have properties which limit the applications for which they can be used. The following subsections review the various radiation types, their interaction with inorganic and organic matter, as well as useful simulation tools which can predict their passage through matter. 


\subsubsection{Radiation Types}

Radiation can be broken down into four types. [19]

Charged particle radiation $\left\{\begin{array}{l}\text { Fast electrons and positrons }\left(\beta^{+} \text {and } \beta^{-}\right) \\ \text {Heavy charged particles including } \alpha \text { particles }\end{array}\right.$

Uncharged radiation $\left\{\begin{array}{l}\text { Electromagnetic radiation including X-ray and } \gamma \text {-radiation } \\ \text { Neutrons }\end{array}\right.$

Though disallowed in classical physics, quantum mechanics allows for $\alpha$ particle tunneling through the potential barrier established by an atomic nucleus. This tunneling, sometimes referred to as barrier penetration, is the mechanism of $\alpha$ decay. Equation 2.1 shows the nuclear equation for $\alpha$ decay where $X$ is the parent, and $Y$ is the daughter (or progeny) nuclei [19]. Emissions are usually mono-energetic with energies in the range of $4-6 \mathrm{MeV}$. At $\alpha$ energies below $4 \mathrm{MeV}$ the probability of barrier penetration is low. Correspondingly, isotopes with shorter half-lives tend to have higher energy emissions. As $\alpha$ particles are reasonably massive, a significant amount of energy is transferred to the progeny and it is termed a recoil nucleus. An $\alpha$ particle is equivalent to a doubly-ionized helium nucleus carrying a charge of +2 .

$$
{ }_{Z}^{A} X \rightarrow{ }_{Z-2}^{A-4} Y+{ }_{2}^{4} \alpha
$$

Beta $(\beta)$ decay includes both electrons $\left(\beta^{-}\right)$and positrons $\left(\beta^{+}\right)$. Equations 2.2 and 2.3 show forms of $\beta$ decay where $\nu$ and $\bar{\nu}$ are a neutrino and anti-neutrino respectively. For all practical purposes the neutrinos and anti-neutrinos are undetectable. Due to 
the low mass of the $\beta$ particle itself, the progeny has little recoil energy. $\beta^{-}$and $\beta^{+}$ carry charges of -1 and +1 , respectively.

$$
\begin{aligned}
& { }_{Z}^{A} X \rightarrow{ }_{Z+1}^{A} Y+\beta^{-}+\bar{\nu} \\
& { }_{Z}^{A} X \rightarrow{ }_{Z-1}^{A} Y+\beta^{+}+\nu
\end{aligned}
$$

Gamma $(\gamma)$ radiation is produced by excited nuclei during their transition to lower-lying nuclear energy levels. As such, there is no change in atomic number. Equation 2.4 presents the decay equation for $\gamma$ particles. Some possible sources of $\gamma$ radiation include de-excitation of a nuclear state of the parent, as well as annihilation reactions between $\beta^{+}$and $\beta^{-}$particles.

$$
{ }_{Z}^{A} X \rightarrow{ }_{Z}^{A} Y+{ }_{0}^{0} \gamma
$$

To complete the list of radiation types, we note neutrons $(n)$ can be produced from spontaneous fission (common in transuranic elements), transmutation reactions $(\alpha$ and $\mathrm{Be})$, photoneutron sources ( $\gamma$ particle striking a suitable target), and reactions of accelerated charged particles (deuterium-deuterium and deuterium-tritium collisions).

\subsubsection{Interaction of radiation with matter}

Knowledge of how radiation interacts with matter is crucial when developing both applications and detectors. This topic has been investigated extensively, and what follows is a brief introduction into the nature of radiation interactions [19,20].

Table 2.1 lists the types of radiation along with the characteristic distance they penetrate solid matter. Charged particles interact primarily via Coulomb forces between their own charge and the orbital electrons in the absorbing material. Nuclear 


\begin{tabular}{ccc} 
Charged Particle Radiation & Uncharged Radiation \\
\hline \hline Heavy charged particles & $\leftarrow$ & Neutrons \\
characteristic distance $10^{-5} \mathrm{~m}$ & & characteristic distance $10^{-1} \mathrm{~m}$ \\
\hline Fast electrons & $\leftarrow$ & $\begin{array}{c}\text { X-rays and } \gamma \\
\text { characteristic distance } 10^{-3} \mathrm{~m}\end{array}$ \\
\hline
\end{tabular}

Table 2.1: Characteristic distance of charged and uncharged radiation. [19]

interactions are possible but less probable. Alternatively, uncharged radiation tends to experience catastrophic interactions more frequently involving the nucleus of absorber atoms where the interaction drastically alters the nature of the incident radiation in a single event. The typical result of these catastrophic interactions is shown in the table as an arrow. In the case of neutron radiation, the neutrons react with target atoms to form unstable $\alpha$ emitting radioisotopes, whereas in X-ray and $\gamma$ radiation there is typically a conversion to fast electrons.

What follows is a brief introduction to the interaction of each type of radiation with matter with a focus on $\alpha$ and $\beta$ radiation.

\section{Interaction of $\alpha$ particle radiation with matter}

Upon entering an absorber, an $\alpha$ particle will interact simultaneously with many orbital electrons and each interaction transfers energy from the $\alpha$ to an orbital electron. Depending on the amount of energy transfered the electron is either left in an excited state, or is freed from the atom (ionized). The net effect on the $\alpha$ particle is to continuously reduce its velocity until it is completely stopped. As the $\alpha$ particle slows, it spends more time in the vicinity of the orbital electrons and the rate at which it loses its energy increases. The rate at which a particle loses its energy as a function

of path length is referred to as the linear stopping power, unrestricted linear energy transfer (LET), or specific energy loss. The classical expression for specific energy 


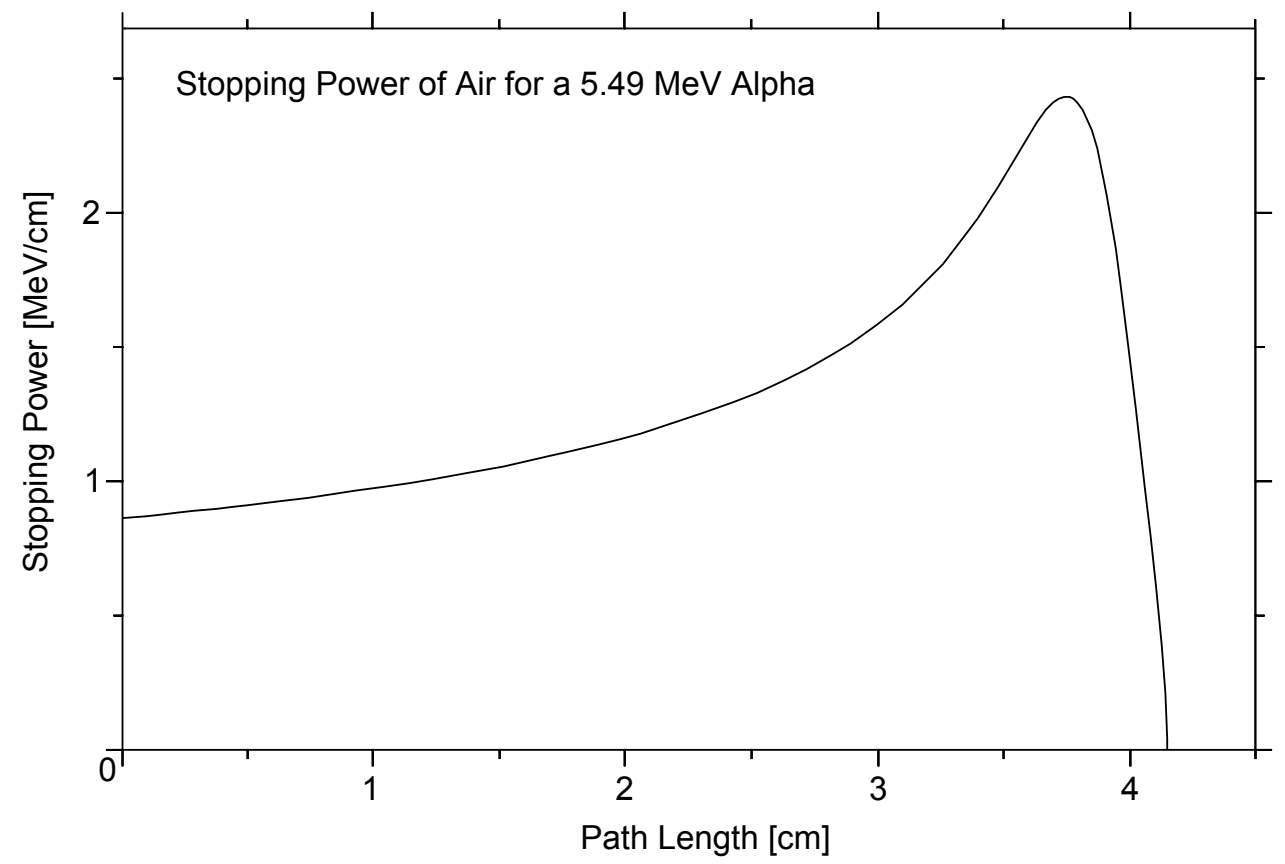

Figure 2.1: Bragg curve for a $5.49 \mathrm{MeV} \alpha$ particle in air.

loss was developed by Bethe [19] and is shown in equation 2.5 below.

$$
S=-\frac{d E}{d x}=\frac{4 \pi e^{4} z^{2}}{m_{0} v^{2}} N Z\left[\ln \frac{2 m_{0} v^{2}}{I}-\ln (1-\beta)-\beta^{2}\right]
$$

Here $e$ is the electron charge, $z$ and $v$ are the charge and velocity of the primary particle, $m_{0}$ is the electron rest mass, $N$ and $Z$ are the number density and atomic number of the absorber atoms, $\beta$ is defined as $v / c$, and $I$ is the experimentally determined ionization potential of the absorber. The important trends in this expression are for non-relativistic particles that $\mathrm{S}$ varies as $1 / v^{2}$, and is larger for increased atomic charge as well as for material density and atomic number. Equation 2.5 begins to fail at low velocities where the $\alpha$, or other heavy charged particle, begin to pick up charge and eventually become neutral.

Figure 2.1 shows how the stopping power for a $5.49 \mathrm{MeV} \alpha$ particle in air steadily increases as the particle energy is reduced, reaches a maximum and drops rapidly once the particle has been neutralized. This type of figure is known as a Bragg curve. 


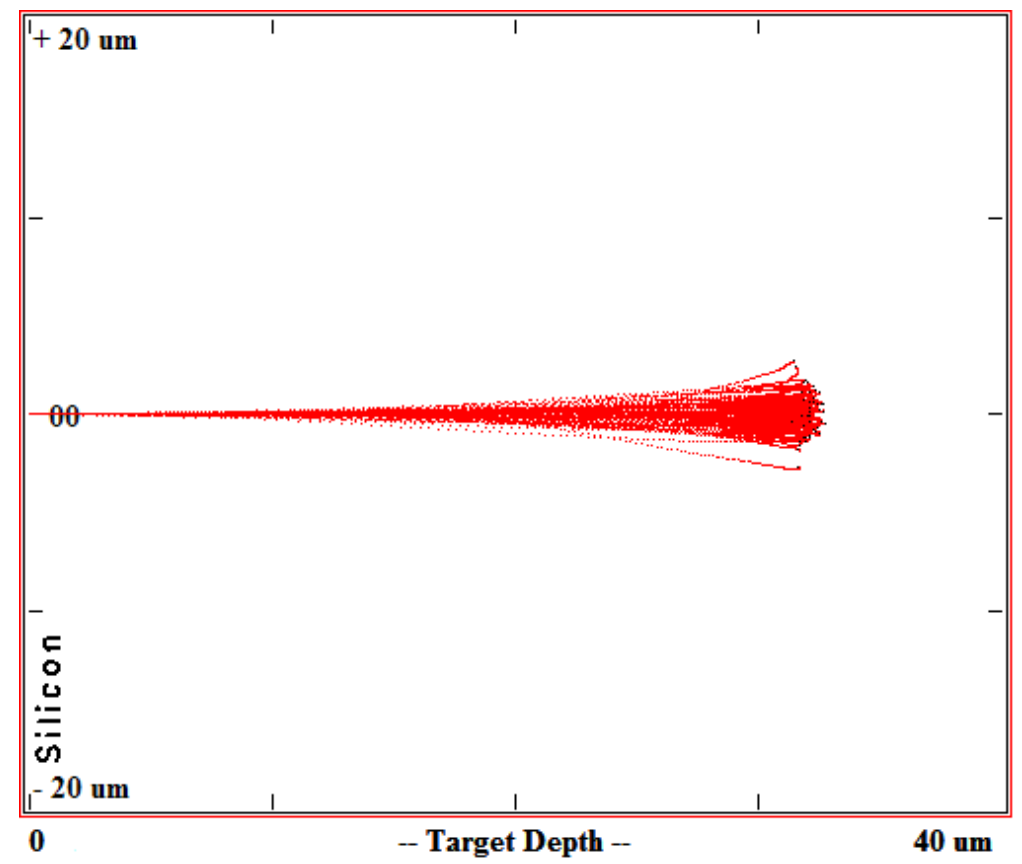

Figure 2.2: Simulation of one hundred $6 \mathrm{MeV} \alpha$ particles in silicon using SRIM2008.

As an $\alpha$ particle is orders of magnitude more massive than an electron, and as the interactions occur simultaneous in many directions, the path taken by an $\alpha$ particle in matter is quite straight. Figure 2.2 shows a simulation of one hundred $6 \mathrm{MeV} \alpha$ particles in silicon using SRIM2008 [21]. Due to these very linear tracks, $\alpha$ particles have a clearly defined range in matter. For silicon, a $6 \mathrm{MeV} \alpha$ has a range of approximately $32 \mu \mathrm{m}$. The variation in encounters causes some variability in the range, and this is termed range straggling.

The primary energy loss mechanism for $\alpha$ particles in matter is ionization. The ion pairs created in the absorber form the fundamental detection mechanism used in many different detectors. It is also important to note that in particularly close encounters between an $\alpha$ and orbital electron, the ionized electron can carry sufficient energy to ionize other atoms. These secondary electrons are also referred to as $\delta$ rays. 


\section{Interaction of $\beta$ particle radiation with matter}

As is the case for other charged particle radiation, $\beta$ particles interact primarily via Coulomb force interaction with the orbital electrons in the absorber. However, unlike the case for $\alpha$ particles, and depending on the energy of the $\beta$ particle, the mass of the orbital electron and $\beta$ are comparable. In general a much larger fraction of the $\beta$ particle's energy can be transfered in a single interaction, and electron-nuclear interactions can result in dramatic changes in the $\beta$ particle's direction. The net result

is a far more randomized path as the $\beta$ particle passes through the absorber. Figure 2.3 presents simulation result of three normally incident $1 \mathrm{MeV} \beta$ particles passing through a slab of silicon. Note the non-linear path and points of large deflection. Backscattering needs to be considered when detecting and analyzing $\beta$ radiation. Typically the secondary ionizing electrons, or $\delta$ rays, do not have particularly long path lengths, but occasionally the energy transfer is sufficient to generate a lengthy path of secondary ion pairs as shown in the simulation. Figure 2.3 was generated using GEAnt4.10, a Monte Carlo toolkit for the simulation of particles passing through matter. Geant4 will be discussed at length in Section 2.1.4.

Stopping power for $\beta$ particles is less than that of $\alpha$ particles. Alongside collisional losses due to Coulomb interactions, there can also be radiative losses as the charge radiates energy when deflected (accelerates). Hence the stopping power, derived by Bethe, is written as

$$
\frac{d E}{d x}=\left(\frac{d E}{d x}\right)_{c}+\left(\frac{d E}{d x}\right)_{r}
$$

where the subscripts $c$ and $r$ indicate collisional and radiative losses respectively. Analytic expressions for the collisional and radiative stopping powers are given in equations 2.7 and 2.8. For $\beta$ energies below several $\mathrm{MeV}$ radiative losses constitute only a small fraction of the energy loss, whereas in high-energy physics (HEP), the 


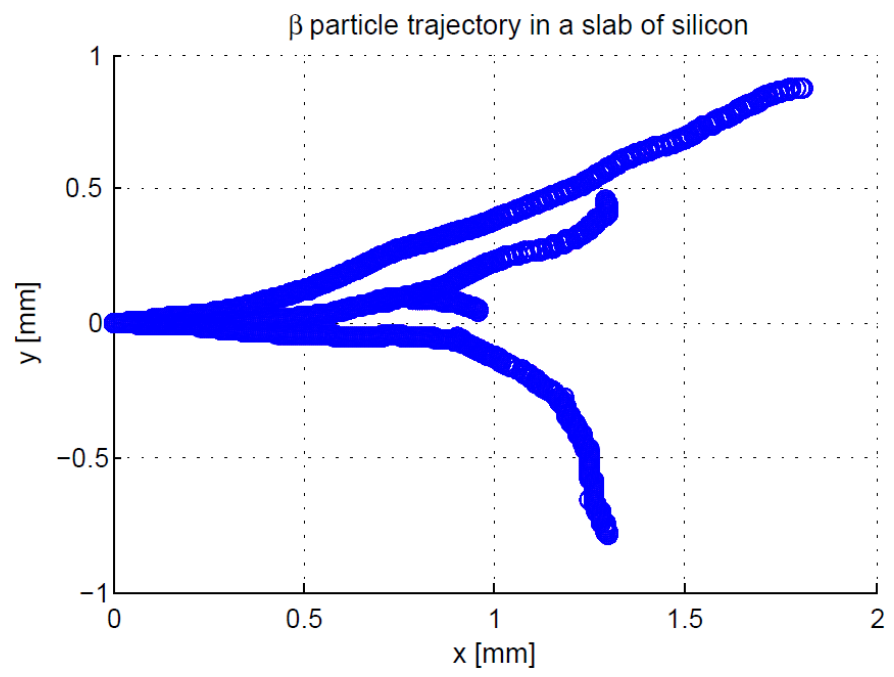

(a) XY Projection

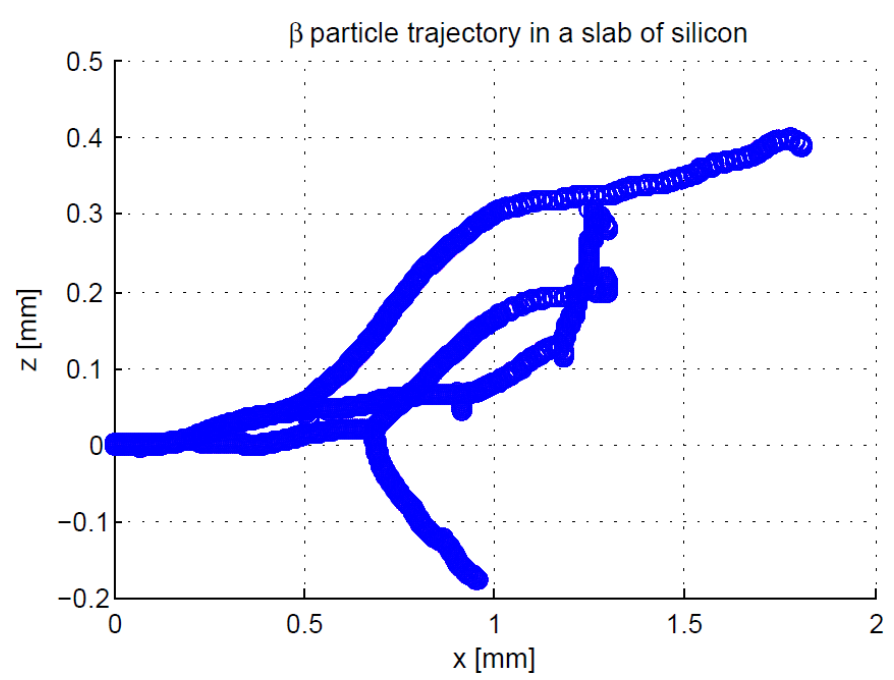

(b) XZ Projection

Figure 2.3: GEANT4 simulation of three normally incident $1 \mathrm{MeV} \beta$ particles passing through a silicon slab starting at the origin. Each point represents the final position of an ionized electron. The YZ plane forms the slab's surface, while the $\mathrm{X}$ axis is depth.

radiative losses, also know as Bremsstrahlung radiation, constitute the dominant energy loss mechanism.

$$
\begin{aligned}
&-\left(\frac{d E}{d x}\right)_{c}= \frac{2 \pi e^{4} z^{2}}{m_{0} v^{2}} N Z\left[\ln \frac{m_{0} v^{2} E}{2 I^{2}\left(1-\beta^{2}\right)}-(\ln 2)\left(2 \sqrt{1-\beta^{2}}-1+\beta^{2}\right)\right. \\
&\left.+\left(1-\beta^{2}\right)+\frac{1}{8}\left(1-\sqrt{1-\beta^{2}}\right)^{2}\right] \\
&-\left(\frac{d E}{d x}\right)_{r}=\frac{N E Z(Z+1) e^{4}}{137 m_{0}^{2} c^{4}} N Z\left[4 \ln \frac{2 E}{m_{0} c^{2}}-\frac{4}{3}\right]
\end{aligned}
$$

An interesting case to be analyzed is that of a thin absorber, for example a thin solid state detector. In this situation the $\beta$ particle's energy loss follows that of a Landau distribution $[22,23]$. This asymmetric distribution is characterized by a single peak followed by long tail and is depicted in Figure 2.4. By observing the form of the distribution it is clear that the mean energy loss will be larger than the most 


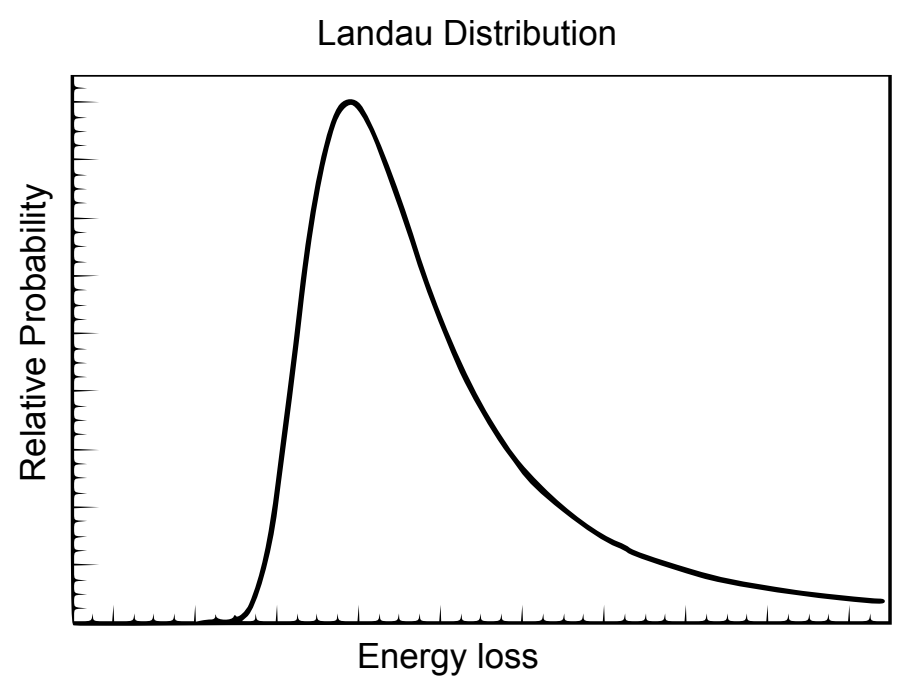

Figure 2.4: General form of the Landau distribution.

probable due to the long tail extending to higher energy loss. The long tail exists due to the probability of large energy transfers in a single interaction. For a thicker absorber there are a larger number of interactions and by the Central Limit Theorem, the energy deposition approaches a Gaussian function. Landau solved the energy loss distribution for a subset of conditions by assuming, among other things, that the maximum energy transfer in any collision was infinite and the electron binding energy is negligible (all electrons treated as free). Equation 2.9 shows the form of the distribution subject to Landau's simplifications where $x$ is the thickness of the absorber and $\Delta$ is the energy loss.

$$
f_{L}(x, \Delta)=\frac{\varphi(\lambda)}{\xi}
$$

where $\varphi(\lambda)$ is a universal function given by

$$
\varphi(\lambda)=\frac{1}{\pi} \int_{0}^{\infty} e^{(-\pi y / 2)} \cos (y \ln y+\lambda y) d y
$$

and 


$$
\lambda=\frac{\Delta-\langle\Delta\rangle}{\xi}-\beta^{2}-\ln (k)-1+C_{E}
$$

where $C_{E}=0.5772, k=\langle\Delta\rangle / W_{m}$, where $W_{m}$ is the maximum energy transfer, and $\beta=v / c$ as before.

The quantity $\xi$ is given by [24]

$$
\xi=\frac{2 \pi z^{2} e^{4}}{m_{0} c^{2} \beta^{2}} \frac{N Z x \rho}{A}=153.4\left(\frac{z^{2}}{\beta^{2}}\right)\left(\frac{Z}{A}\right) x \rho
$$

where $N$ is Avogadro's number, $m_{0}$ and $e$ are the electron mass and charge respectively, $z$ is the charge on the incident particle, $Z, A, \rho$, and $x$ are the atomic number, atomic weight, density, and the absorber thickness.

A number of additions to this function have been made by Vavilov, Blunck and Leisegang, Shulek, and Bichsel [25-28]. For example Vavilov included limited energy transfer upon collision. Others added the condition of electron binding energy.

In practice, for solid state material, a broadening to the Landau distribution is seen and experiments have also shown the electronic noise cannot be solely responsible. This is particularly the case for very thin absorbers [29]. By using a modified cross section which takes into account the electron binding energy, the energy loss function can be written as in Equation 2.13 [24].

$$
f(x, \Delta)=\frac{1}{\sigma \sqrt{2 \pi}} \int_{-\infty}^{\infty} f_{L}(x, \Delta-\tau) e^{-\frac{\tau^{2}}{2 \delta_{2}}} d \tau
$$

Shulek proposed Equation 2.14 as the form for $\delta_{2}$ where $I_{i}$ if the effective ionization potential of the $i$-th shell and where $f_{i}$ is the fractions of electrons in that shell.

$$
\delta_{2}=\frac{8}{3} \frac{\xi}{x} \sum_{i} I f_{i} \ln \frac{2 m_{0} c^{2} \beta^{2}}{I_{i}}
$$

The end result is a convolution of the Landau distribution with a Gaussian function 
leading to a broadening in the shape of the energy loss. As the material thickness decreases the $\delta_{2}$ term has a more prominent effect on the distribution.

\section{Interaction of $\gamma$ radiation with matter}

Many mechanisms for $\gamma$ ray interaction exist, but in practice photoelectric absorption, Compton scattering, and pair production are the most significant [19]. All of these interactions result in either a partial, or complete transfer of $\gamma$ energy to electron energy. Recall Table 2.1 showed an arrow pointing towards the generation of fast electrons. This is a significant change from both $\alpha$ and $\beta$ radiation which slow down more gradually through Coulomb interactions with matter.

In photoelectric absorption, the $\gamma$ ray is completely absorbed by the atom and the result is a free electron whose energy is the difference between the $\gamma$ particle energy and the binding energy of the original electron shell. The most probable origin of the photoelectron is the K-shell and a marked increase in interaction rate is seen for $\gamma$ energies above this level. In addition to the generated photoelectron, an X-ray, or even Auger electron, can be generated as another free electron fills the vacancy created by the $\gamma$ ray. Generally these X-rays are self-absorbed and need not be considered. Photoelectric absorption interaction rates vary strongly with both atomic number $(Z)$ and $\gamma$ energy $\left(E_{\gamma}\right)$. Equation 2.15 below provides a rough approximation for the probability $(\tau)$ of a photoelectric absorption where $n$ varies between 4 and 5 [19].

$$
\tau \approx \text { constant } \times \frac{Z^{n}}{E_{\gamma}^{3.5}}
$$

Compton scattering results in partial transfer of energy from the incident $\gamma$ to an electron as depicted in Figure 2.5. Here the electron which was initially assumed to be at rest is sent off at an angle $\phi$ and is termed a recoil electron. As all angles of scattering are possible the energy transfer can range from minimal to a large portion 


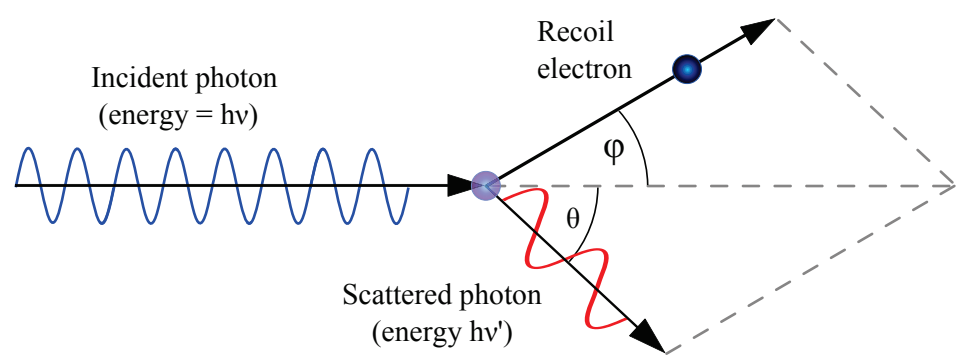

Figure 2.5: Compton scattering.

of the $\gamma$ energy. Equation 2.16 can be used to calculate the energy of the scattered photon provided the scattering angle is known.

$$
h \nu^{\prime}=\frac{h \nu}{1+\frac{h \nu}{m_{0} c^{2}}(1-\cos \theta)}
$$

The probability of Compton scattering depends on the number of electrons and varies linearly with atomic number. The angular distribution is predicted by the Klein-Nishina formula [19].

Lastly, pair production becomes possible when the $\gamma$ energy is twice that of the electron rest mass, or $1.022 \mathrm{MeV}$. Yet, the probability of pair production remains low until incident photon energies of at least several $\mathrm{MeV}$ are reached. In pair production an electron and positron pair are created which share the energy of the incident photon. Upon slowing down, the positron will annihilate with an electron and produce a secondary $\gamma$ ray.

\section{Interaction of neutron radiation with matter}

Neutrons do not experience Coulomb forces as charged particles do. They react with the nuclei of their absorber in a variety of ways, often producing heavy charged particles. For low-energy (slow) neutrons, elastic scattering and neutron-induced reactions dominate. Elastic scattering is probable but imparts little energy to the 
nucleus and serves simply to slow the neutron down to thermal equilibrium at which point neutron-induced reactions take place. These reactions can include $(\mathrm{n}, \gamma),(\mathrm{n}, \alpha)$, $(\mathrm{n}, \mathrm{p})$, and (n,fission). Detecting neutrons is often accomplished by observing the charged secondaries created.

For high-energy (fast) neutrons neutron-induced reactions are less probable and scattering effects dominate. Recoil nuclei can be created and detected if the neutron imparts sufficient energy. At high energy, inelastic scattering is also possible where the absorbing nucleus becomes excited, and then relaxes emitting a $\gamma$ ray. The additional energy necessary to excite the nucleus comes at the expense of neutron energy and as a result, inelastic scattering results in more energy loss than a purely elastic collision.

\subsubsection{Biological effects of ionizing radiation}

Exposure of biological matter to ionizing radiation sets off a chain of events which can be grouped as radiochemical, biochemical, and biological effects. Radiochemical effects occur within microseconds of exposure with the generation of (bio)radicals. Biochemical effects follow within minutes with the alteration of biological structures and cell membranes. Lastly, biological effects such as apoptosis (programmed cell death), cell death by other avenues, and genetic mutations become evident within hours or longer [30].

As ionizing radiation passes through living biological matter the energy deposited can result in single and double DNA strand breaks in the cell nucleus. Single-strand breaks (SSB) can be easily repaired. However, double-strand breaks (DSB), if not properly repaired by the cell, can lead to chromosomal aberrations that may eventually be lethal to the cell. Additional damage in the cell membrane can also lead to cell death $[30,31]$. If cell death does not occur, the double-strand breaks can lead to genetic mutations which can have long term repercussions. 
Modification of DNA bases comprise the majority of radiation-induced cell damage. Should the density of modification be sufficiently high, the cell can no longer effectively repair the damage. With two single-strand breaks spaced less than 14 base pairs apart, a DNA double-strand break is created which cannot readily be repaired [32]. Ionizing radiation with high LET (such as $\alpha$ particles) produces a higher density of radiation-induced damage and is then particularly cytotoxic to the cell [33]. In fact the passage of a single $\alpha$ particle can lead to cell death, while it would take three to four orders more $\beta$ particles to produce similar effects $[30,34]$.

\subsubsection{Simulation tools}

Due to the complexity and stochastic nature of radiation interactions, analytical solutions are often impractical. Monte Carlo simulations lend themselves well to this situation where a large number of events are simulated and trends in the results observed. A number of simulation tools exist for the passage of radiation through matter and a short listing of tools with basic descriptions follows.

- CASINO (monte CArlo SImulation of electroN trajectory in sOlids) was written with an emphasis on low-energy electron beam simulations in scanning electron microscopy $[35,36]$.

- MCNP (Monte Carlo Neutron Photon) was developed at the Los Alamos National Laboratory and was written for simulating both neutron and photon transport [37].

- SRIM (Stopping Range of Ions in Matter) provides the ability to perform simulation of heavy charged particle passing through matter [21].

- PENELOPE (PENetration LOss of Positrons and Electrons) performs Monte Carlo simulation of coupled electron-photon transport in arbitrary materials and 
covers an energy range from a few hundred eV to approximately $1 \mathrm{GeV}$ [38].

- EGSnrc (Electron Gamma Shower) was developed at the National Research Council in Canada and uses Monte Carlo methods to simulate electron and photon transport in matter with an emphasis on medical physics simulations $[39]$.

- GEANT (GEometry ANd Tracking) was developed by CERN and is a full featured Monte Carlo simulation toolkit written in object oriented $\mathrm{C}++$. The toolkit is provided as a series of base classes which users must compile into their own code. Areas of application include High Energy Physics (HEP), accelerator physics, as well as medical and space applications. GEANT4 is the current version of the toolkit $[14,15]$.

For the bulk of the work presented herein GEANT4 was chosen for simulation. This particular toolkit is robust, well-featured, and through specialized microelectronics models, provides accurate modelling of interactions to near the ionization energy of silicon.

It should be noted that none of the tools listed above allow for electron and hole transport in a semiconductor target. Some semiconductor simulation tools such as Medici [40] and Sentaurus [41] do provide very limited simulation ability for radiation induced effects. Both Medici and Sentaurus allow columnar tracks of electron-hole pair generation to be introduced for the simulation of $\alpha$ particle strikes, but neither provides robust modelling of $\beta$ particles. Sentaurus also includes some ability to simulate $\gamma$ radiation. 


\subsection{Radiation Detectors}

From photographic film, micro channel plates (MCP), to the Nobel prize winning work by Charpak in 1968 on multi-wire proportional counters (MWPC) [42], radiation detector development has a rich history with many fascinating detection schemes. A good starting point in this discussion would be the book by Knoll [19]. Semiconductor, particularly silicon based, detectors have recently proliferated the field of charged particle detection and this is the detection scheme used in this work. For this reason this review will be limited to silicon with an emphasis on MOS based sensors. Before elaborating on the operation of these detectors a brief review of semiconductor fundamentals follows. A PN junction diode will be used as an example during the review. The books by Sze, Tsividis, and Grove offer a far more complete description of semiconductor principles [43-46].

\subsubsection{Semiconductor Basics and the PN Junction Diode}

Crystalline silicon (c-Si) is an indirect semiconductor residing in column IV of the periodic table. At room temperature its bandgap is $1.11 \mathrm{eV}$ and pure silicon, also known as intrinsic silicon, is a poor conductor. At absolute zero $(0 \mathrm{~K})$, all the silicon atoms in the crystal would have full valence shells and there are no free electrons available for conduction. As thermal energy increases more and more electrons acquire enough energy to jump from the valence band to the conduction band and are free to participate in conduction. Note that as these electrons transition to the conduction band, they leave behind their ionized parent nucleus and what is referred to as a "hole", or an absence of an electron. These holes are free to move about the crystal lattice as well.

By doping the silicon with a pentavalent impurity, like phosphorus, it is possible to increase the number of free electrons available for conduction. Phosphorus, having 
five valence electrons, introduces an energy level just below silicon's conduction band and little energy is required to ionize the phosphorus and promote a free electron available for conduction. Here phosphorus is termed a donor as it donates a free electron. Having donated an electron, a positively charged phosphorus ion is left behind. Silicon doped with a pentavalent impurity, an electron donor, is called $\mathrm{N}$ type. Alternatively, a trivalent impurity, like boron, can be used which introduces an energy level just above the valence band which readily accepts an electron. Here the boron introduces a hole and a negatively charged boron ion. Material doped with a trivalent impurity, an electron acceptor, is called P-type.

As shown in Figure 2.6, when P-type and N-type silicon are brought into contact a region known as a depletion region, or space charge region, is formed. Due to concentration gradients on each side of the junctions, electrons from the N-type region diffuse into the P-type region, while holes in the P-type region diffuse into the N-type. These diffused carriers will quickly recombine, and the net effect is the production of the depletion region. This region will continue to widen until a built-in electric field large enough to inhibit further diffusion is established. It is important to note that the carriers which have diffused will have left behind their ionized dopant atoms which are immobile in the silicon crystal. The term depletion region refers to the depletion of mobile charge carriers from this region.

When an electron and hole meet they recombine and no longer participate in conduction. In the N-type region, electrons are the majority charge carriers, while holes are the minority charge carriers. Alternatively in the P-type region, holes are the majority charge carriers, while electrons are the minority charge carriers. In either region, the amount of time, and the distance a minority carrier will travel, are called the minority carrier recombination lifetime and the minority carrier diffusion length, respectively. In the absence of an electric field, minority carriers will diffuse in a random fashion until they recombine. The amount of diffusion, and the recombination 


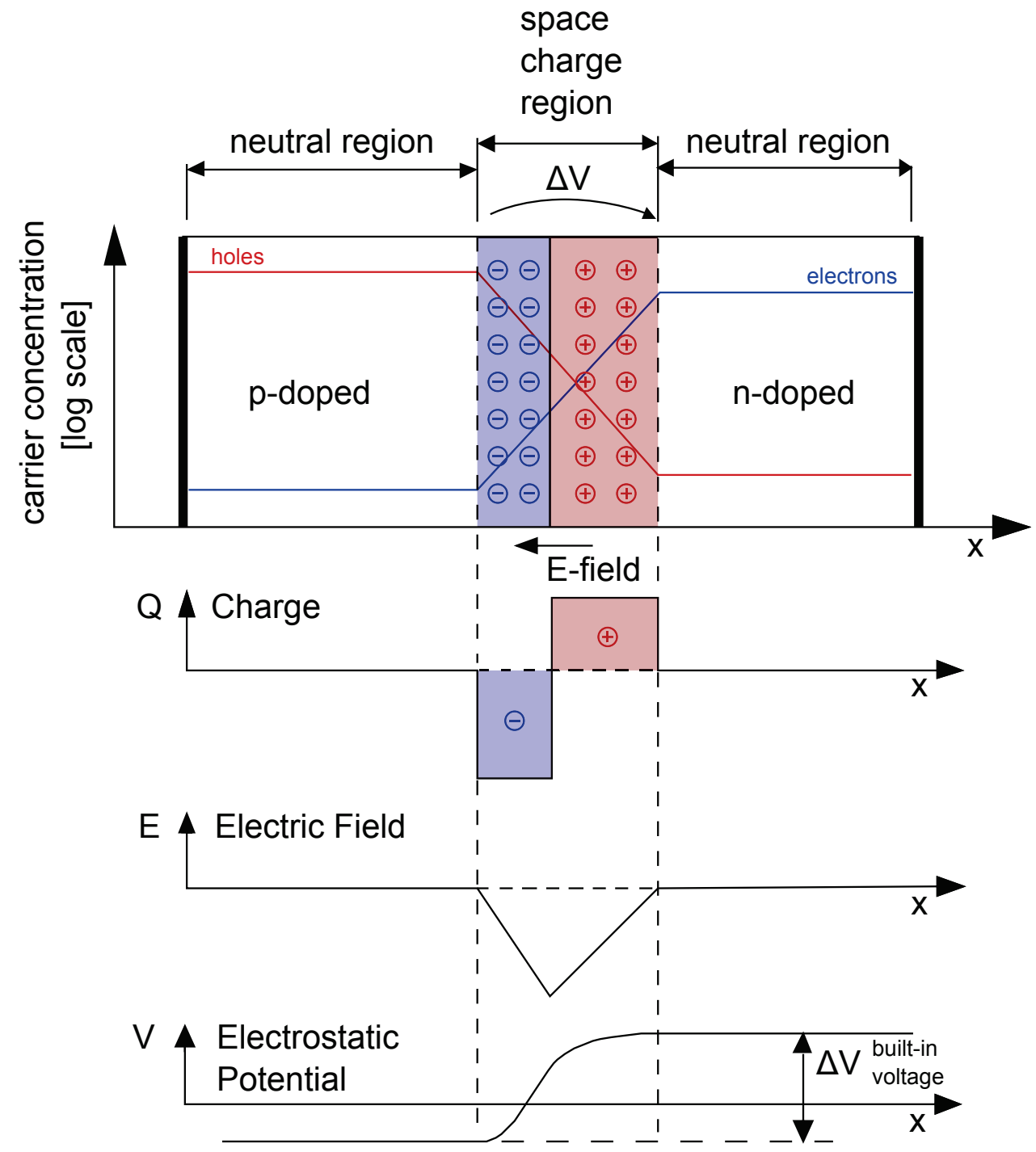

Figure 2.6: Silicon PN junction. 
lifetimes, will be different for each type of carrier as well as a function of the mobility, impurity concentration, temperature, and defects in the crystal. Should a free carrier diffuse to, or be generated in, an electric field, the field will cause the charge carrier to drift in the direction consistent with the field.

There is a relationship between charge stored in, and the width of, the depletion region junction. This leads to a junction capacitance which, for the case shown in Figure 2.6, can be modelled as parallel plate capacitor where the separation between the plates is given by the depletion region width. The depletion region width will increase under increasing reverse bias resulting in a lower junction capacitance.

Much more could be said, but having introduced some basic semiconductor concepts, as well as the PN junction diode, a discussion of the various types of silicon detectors can be had.

\subsubsection{Silicon Radiation Detectors}

Over half a century of refinement and enormous investments have made silicon a mature process technology. This has led to the development of many different types of silicon based radiation sensors. Popular examples include PIN diodes [19], silicon strip detectors [47], silicon photomultipliers (SiPM) [48], hybrid detectors [10,11,49], charge coupled device (CCD) detectors [47], and complementary metal oxide semiconductor (CMOS) pixel sensors [50-56]. These sensors can be used to detect ionizing radiation directly provided the radiation is able to reach the sensitive volumes of the detectors. In some cases radiation can be detected indirectly by adding a scintillating material on or near the detector itself. A short description of each type of sensor follows.

- PIN diodes are a type of PN junction diode where the junction between the Ptype and N-type material is nearly intrinsic. This allows the for a wide depletion region, and a very low junction capacitance. This type of detector can provide 
very good energy resolution, but does not provide any location information on the point of interaction of the incident radiation.

- Silicon strip detectors are long strips of PN junction diodes in a single substrate.

This type of detector can be run with a large reverse bias to aid in charge collection and provide one dimensional (1D) information on the location of the interaction. Double sided silicon strip detectors (DSSSD) are also possible where the long strips of PN junction diodes are implanted orthogonally on either side of the silicon substrate allowing for two dimensional (2D) strike information to be measured.

- Silicon photomultipliers are 2D arrays of PN junction diodes engineered such that the electric field is large enough to produce an avalanche of additional charge carriers much in the same way as a Geiger-Muller tube.

- Hybrid detectors are a class of detector where a slab of semiconducting material, not necessarily silicon, is bump bonded, or deposited, atop a pixelated readout integrated circuit. Having the detector and electronics separate allows both to be optimized individually, but increases the cost of the detectors. They provide very good sensitivity and are popular in high energy physics. The semiconductor material often has a large field applied to improve the collection efficiency.

- Charge couple devices (CCD) and Complementary metal oxide semiconductor (CMOS) detectors will be covered in more detail below.

All the silicon-based sensors listed above rely on the collection of free charge carriers created by incident ionizing radiation. As described in Section 2.1 ionizing radiation reaching, and interacting with, the sensitive portions of these detectors can promote valence band electrons to the conduction band provided the energy of the incident radiation is sufficiently high. For silicon, on average, $3.6 \mathrm{eV}$ of energy is 
required to generate an electron-hole pair [57]. An electric field separates and collects electron-hole pairs allowing the interaction to be observed.

CCD and CMOS detectors are commonly used in both scientific and consumer electronics. Both are frequently used as optical imaging devices and with the application of the red, blue, and green (RGB) colour filter, also know as a Bayer layer, can be used to produce colour images. Details about the operation of both CCD and CMOS detectors can be found below.

\section{CCD detectors}

CCDs were originally developed in 1969 at AT\&T as a potential memory storage device, delay line, or image sensor [58]. In 2009 the inventors were awarded the Nobel Prize for Physics for their work on the topic. A long development history has produced image sensors of exceptional quality and they are sometimes referred to as the gold standard in image sensor technology [47]. The devices consist of a pixelated array of overlapping polysilicon electrodes which produce a surface potential to trap photogenerated carriers, as well as shift those carriers across the device for eventual readout by an external amplifier. A number of variations of CCDs are possible, but their fundamentals remain the same. Figure 2.7 presents the cross section of a 3 phase CCD image sensor, while Figure 2.8 demonstrates how readout is performed. Charge is transferred in parallel across the pixel array into a readout register where it is then read out serially using an external amplifier and analog to digital converter (ADC).

By utilizing external readout electronics CCD image sensors can have a minimum of overlying passivation, as well as a substrate tailor-made for optical or ionizing radiation detection. This can include thick, high resistivity, epitaxial layers for maximizing the detector's collection volume. These are advantageous features in a ionizing radiation detector. However, pixel-by-pixel readout results in longer readout operations, and the lateral movement of charge can skew strike location information if the 


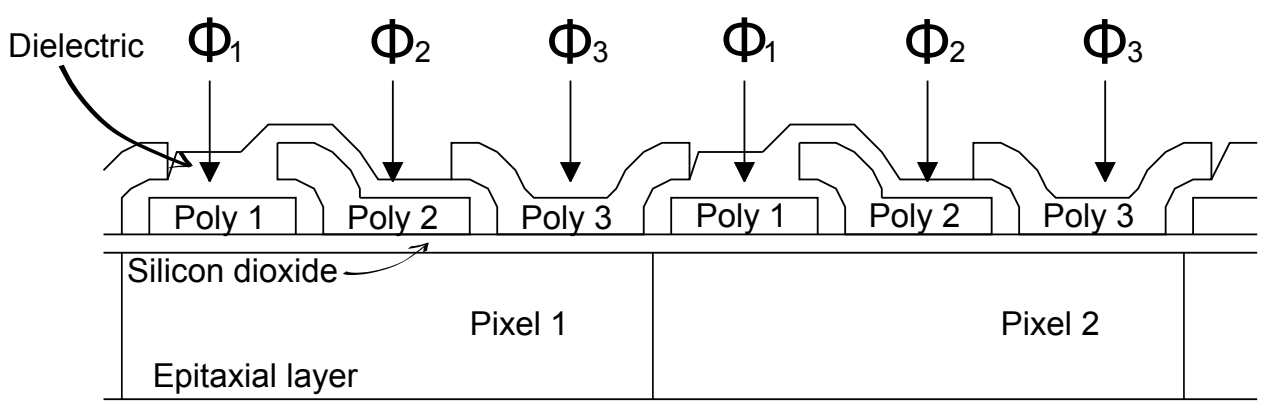

Figure 2.7: CCD cross section.
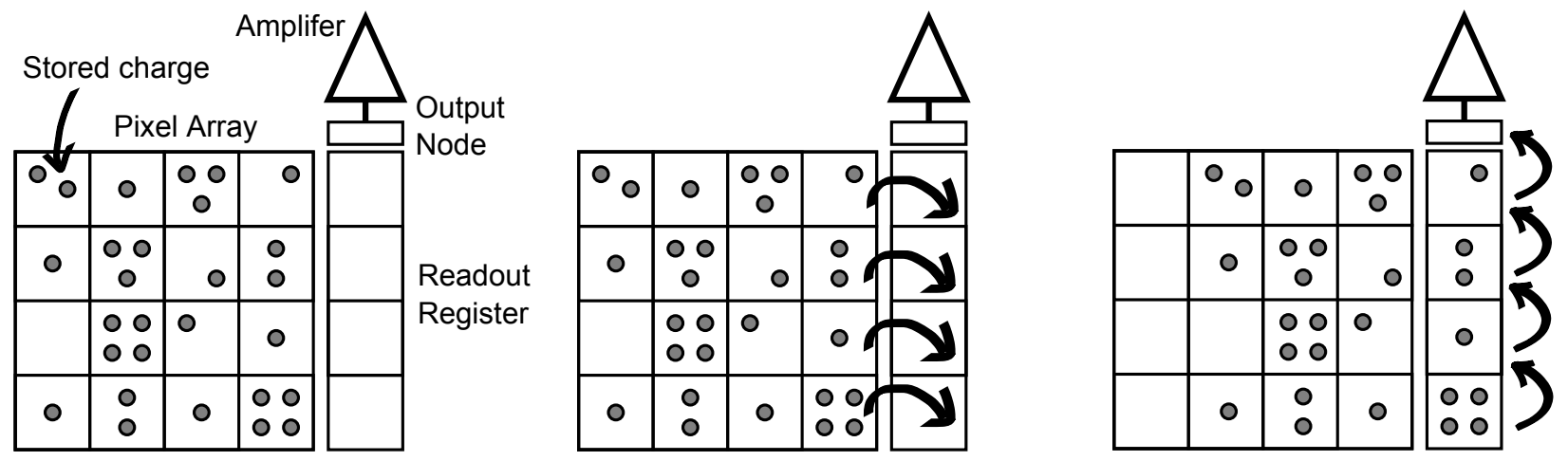

\begin{tabular}{|c|c|c|c|}
\hline${ }^{\circ} \circ$ & 0 & $\begin{array}{c}00 \\
0\end{array}$ & $\begin{array}{l}0 \\
0\end{array}$ \\
\hline 0 & $\begin{array}{c}00 \\
0\end{array}$ & 0 & 0 \\
\hline & $\begin{array}{ll}0 & 0 \\
0 & 0\end{array}$ & 0 & $\begin{array}{lll}0 & 0 \\
0 & 0\end{array}$ \\
\hline 0 & $\begin{array}{l}0 \\
0\end{array}$ & 0 & \\
\hline
\end{tabular}
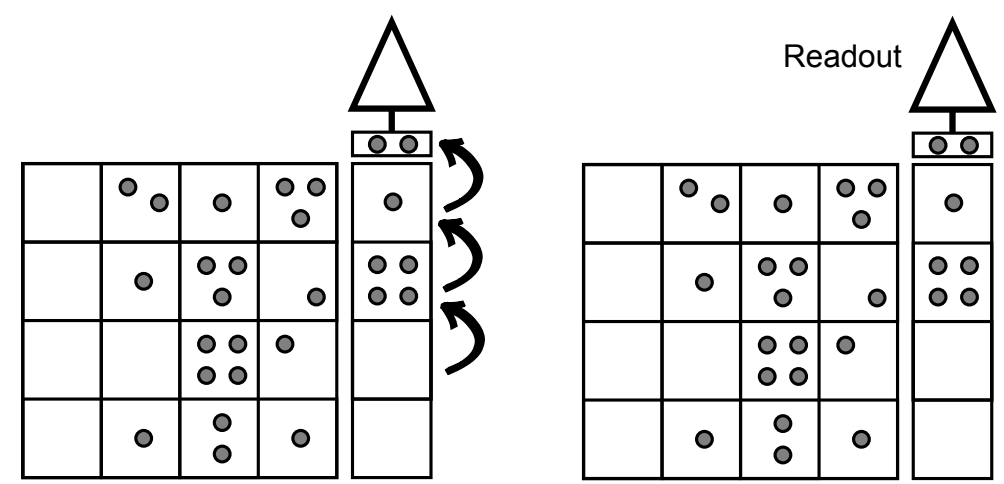

Figure 2.8: CCD operation. 

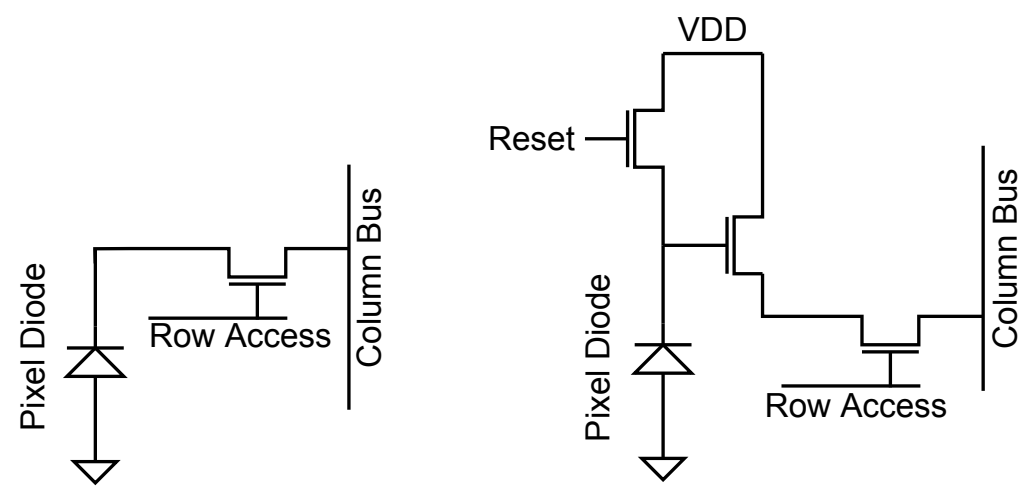

Figure 2.9: Passive and active pixel sensor pixel schematics.

sensor is struck during readout. The minimum amount of time over which the signal can be integrated is given by the sensor's readout time.

\section{CMOS detectors}

Initially developed in the 1990s, CMOS detectors are a less mature technology than CCDs. However, due to recent innovations, they have become comparable in performance to the gold standard of CCD technology [59-63]. CMOS detectors generally consist of a 2D pixelated array of reverse biased PN junction diodes. Unlike CCD detectors however, all the necessary control, amplification, and digitization stages are integrated onto the same silicon substrate. In "passive" detectors each diode is connected to a readout line directly through an access transistor, while in "active" detectors each pixel has a built-in amplification stage. Figure 2.9 presents a pixel schematic for both a passive and active pixel sensor. Variations on the schematics are possible, but the active three transistor (3T) cell is very common. Active pixel sensors are the most common and are often referred to as monolithic active pixel sensors (MAPS) due to the integration of all the control and processing electronics. Pixels are reversed biased and allowed to float electrically. Ionizing radiation interacting with a pixel will cause a drop in charge stored in the junction capacitance.

Figure 2.10 presents the typical readout architecture of a CMOS pixel sensor. 


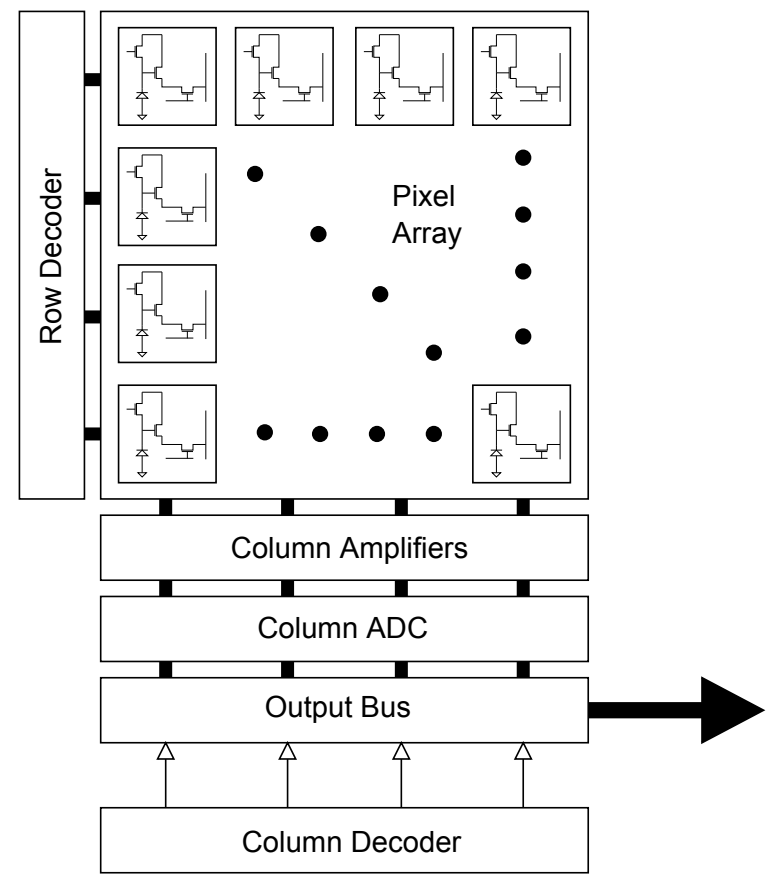

Figure 2.10: CMOS MAPS readout architecture.

Readout of CMOS sensors is often faster than CCD due to parallelization of the readout process with an ADC on each column. Variations exist where ADCs are shared between multiple columns, or with an ADC at each pixel. As each pixel in the detector can be addressed individually, this enables region of interest (ROI) analysis where only a subset of pixels are read further increasing readout speed and reducing the amount of data generated.

Unlike CCDs where the substrate can be optimized purely for detection, CMOS MAPS require a substrate design which allows the on-board electronics to operate properly. In general this results in sensors with lower resistivity substrates and thinner epitaxial layers reducing the charge collection capability. Lower resistivity substrates also tend reduce the depletion region width for a particular bias, and for CMOS MAPS it is sometimes assumed to be zero [47]. An advantage of CMOS MAPS, however, includes lower power dissipation. Less power being consumed also produces less heating reducing thermal noise. Additionally, as CMOS sensors are fabricated in 
relatively conventional foundry processes, they can be mass produced in a very cost effective fashion.

\subsubsection{Noise Sources in Silicon Detectors}

A variety of noises sources will influence the data produced by silicon detectors, and these sources should be considered during analysis. A short list and description of the noise sources observed in CCD and CMOS detectors follows.

\section{Charge transfer efficiency}

CCD operation requires shifting pockets of charge stored on the detector for readout and some charge is lost in this process. Charge transfer efficiencies for CCDs are commonly $99.99 \%$ and better [64].

\section{Shot noise}

Ionizing radiation striking the sensor will release a discrete number of charge carriers in or near the sensitive volume of the detector. When the number of carriers that reach the detector is small a noise component is added. This "shot" noise $\left(\sigma_{\text {shot }}\right)$ obeys Poisson statistics and thus the noise is given by Equation 2.17 where $N$ is the number of charge carriers that reach the detector.

$$
\sigma_{\text {shot }}=\sqrt{N}
$$

\section{Thermal noise and junction leakage}

Thermal noise, also known as Johnson-Nyquist noise, is generated by the random thermal agitation of charge carriers in a conductor which is present even in the absence of an applied bias. 
A biased PN junction will exhibit a small current due to carriers being thermally generated in the depletion region. In the case of a floating reversed biased junction, this current will lower the potential across the junction until equilibrium is reached. This imposes a limit on the maximum possible integration time in CMOS MAPS. This thermally generated current is also known as "dark current". Generation and recombination centers caused by impurities or crystal defects inside the junction have a significant impact on the dark current.

\section{Reset noise}

After the charge stored in a pixel has been digitized, the pixel, or readout capacitor, is reset to a known state. Variations in the reset state potential determine the reset noise of the detector. Channel charge of the reset transistor can be partially stored on the pixel. One way to reduce this reset noise is by controlling the manner in which the reset transistor is driven. A "hard" reset involves driving the reset transistor with a potential larger than the detectors typical supply voltage $\left(V_{\text {resert }}>V_{D D}\right)$ which will introduce a noise of $\sqrt{k T C}$ where $k, T$, and $C$ are Boltzmann's constant, temperature, and capacitance, respectively. Alternatively, a "soft" reset drives the transistor with a potential less than the supply voltage $\left(V_{\text {reset }}<V_{D D}\right)$ and introduces half as much noise, but may not completely reset the pixel. A "flushed" reset involves a hard reset followed by a soft reset which allows the pixel to be completely reset while only introducing $\sqrt{k T C} / 2$ of additional noise.

Another method to reduce the effect of reset noise is correlated double sampling (CDS). CDS measures the charged stored in a pixel immediately after reset, and once the pixel is read. The two measurements are subtracted from one another and yield only the signal integrated in the measurement interval. 


\section{Read noise}

Before the charge stored in a pixel is digitized it can be subject to a number of amplification steps. The noise from all the active and passive electronics along the readout chain including buffers, amplifiers, and ADCs, contributes to the pixel read noise.

\section{Fixed pattern noise}

Non-uniform processing conditions during manufacture of the detectors leads to fixed pattern noise. Some of the non-uniformities include variation in pixel size, doping level, and defects in the silicon crystal. The active electronics also contribute to fixed pattern noise as buffer, amplifier, and ADC performance vary across the wafer. Threshold voltage variation is one phenomenon leading to the variability in electrical response.

\section{Radiation induced noise}

Prolonged exposure to ionization radiation can induce additional noise in detectors by causing damage to the silicon and passivation. Charge generated and trapped in the field and gate oxide can lead to field inversion or changes in transistor performance (threshold voltage shifts). Damage to the silicon crystal will introduce additional generation-recombination centers which can result in increased dark current. Pixelated detectors are in general more radiation hard than large single junction devices. By reducing the junction area, the relative effect of increased dark current is reduced $[57,65,66]$. 


\subsubsection{Limitations on Spatial Resolution in Silicon Detectors}

Scattering of the ionizing radiation, and diffusion of ionized electrons, limit the spa-

tial resolution of silicon-based detectors. Scattering in the overlying passivation can be minimized by reducing the amount of passivation, or by utilizing back-thinned detectors $[51,67]$. In a back-thinned detector the detector's substrate is thinned with only a thin passivation layer present (See Figure 2.11). Back-thinned CMOS detectors are becoming more popular, but their noise performance has yet to reach that of a conventional CMOS detector [47]. Scattering in the silicon itself can lead to loss of spatial resolution, or in some cases, ionizing radiation backscattering out of the silicon may appear as another event.

Diffusion from the point of interaction leads to charge spreading in the substrate. This spreading allows multiple pixels to collect charge generated from a single event, or a single pixel a distance from the original point of interaction. Provided the charge spreading involves at least two pixels, it can be possible to calculate a center of mass of the interaction site and determine the strike location to a sub pixel size level [68].

Charge being collected by an electric field will also continue to diffuse however, the amount of diffusion will be reduced. By increasing the size and strength of the collection electric field, the spatial resolution can be improved. In the extreme, the width of the depletion region can span the entire substrate and result in "fully depleted" detectors. Conversely, some CMOS detectors have a depletion region so small, that the movement of an electron-hole pair generated by the ionizing radiation is assumed to be only from diffusion.

\subsubsection{Example Silicon Sensors}

Some popular examples of 2D silicon detectors include MEDIPIX [10,11,49], MIMOSA [50-52] and the MI3 Vanilla [53]. Other attempts at integrated sensors have 


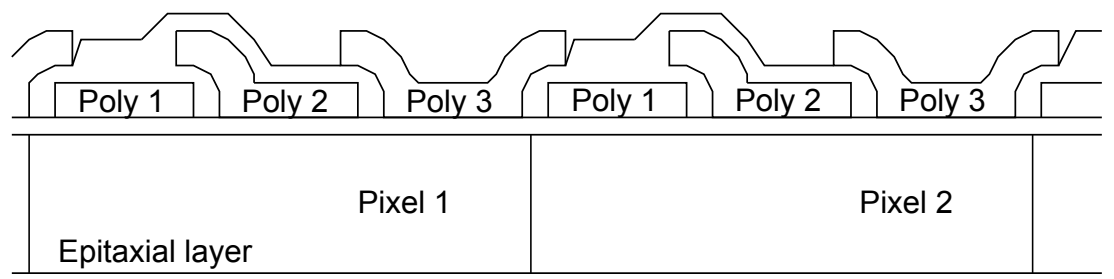

Substrate

(a) Standard CCD detector

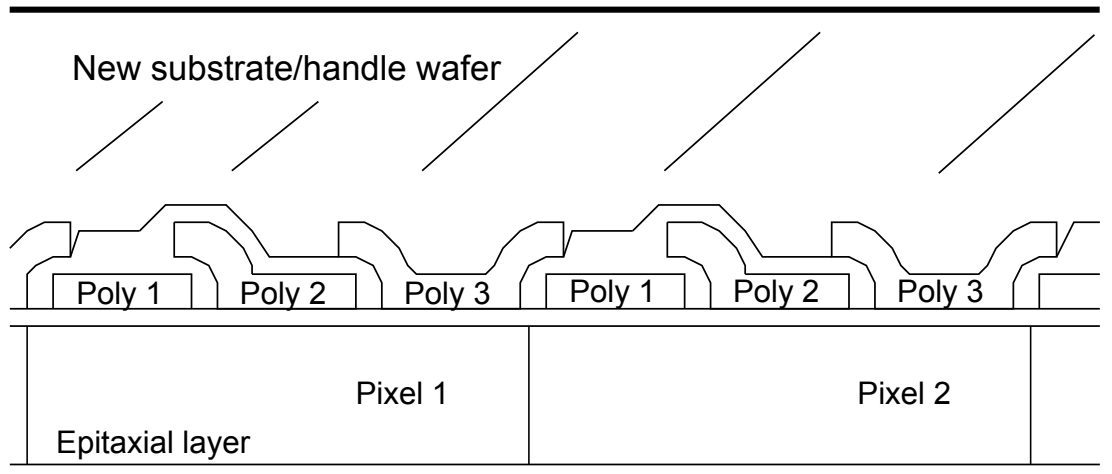

Substrate

(b) Mechanal support attached to surface of a standard CCD detector

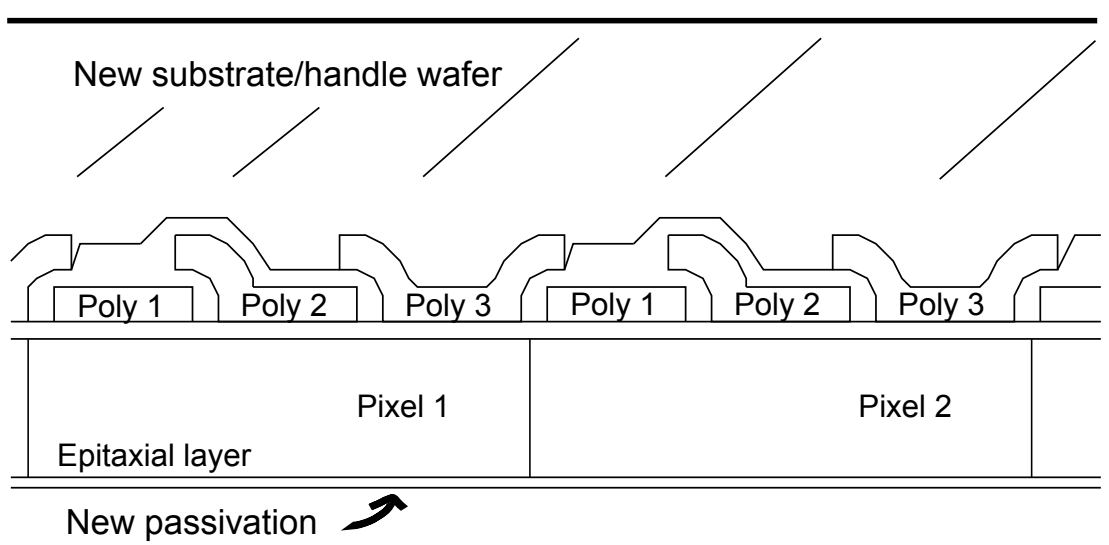

(c) Substrate of the original CCD detector is etched down the epitaxial layer and a passivation layer is grown

Figure 2.11: Back-thinned detector. 
also been made [54-56].

MEDIPIX is a hybrid pixel sensor. It uses a silicon integrated circuit bump bonded to an absorber to detect radioactive emissions. Both silicon and GaAs absorbers have been used. The advantages of this system include stability, high duty cycle and simple offline analysis. Disadvantages include $55 \mu \mathrm{m}$ granularity, lack of analog information and a $3 \mathrm{keV}$ noise floor. [49]

MIMOSA (Minimum Ionizing Particle MOS Active pixel) is a monolithic active pixel sensor in both front and back-illuminated varieties. It was implemented in a $0.6 \mu \mathrm{m}$ CMOS process and an industrial partner handled back thinning the device. The IC has experienced multiple re-designs and is currently in its fifth iteration (MIMOSA V). Advantages include $17 \mu \mathrm{m}$ granularity, full analog output and detectability at $1 \mathrm{keV}$. Disadvantages include large data volume, low duty cycle and non-trivial offline analysis. [49]

Vanilla is a general purpose detector designed as part of the Multidimensional Integrated Intelligent Imaging Project (MI3) collaboration. It offers a $25 \mu \mathrm{m}$ pixel size in a $520 \times 520$ array and is available in a standard or back-thinned variant. The IC has both digital and analog outputs and can be used for region of interest (ROI) analysis. [53]

A variety of other attempts of radiation sensitive ICs have been made. One uses a high voltage CMOS process [54] with deep N-well reverse biased to over $50 \mathrm{~V}$. Another uses a smart-cut SOI approach [55] where the pixel is created in the handle wafer. Another group has developed a thin film on ASIC (TFA) approach where amorphous silicon (a-Si:H) is deposited atop a custom ASIC. This last approach can produce near $100 \%$ fill factor and good radiation hardness, but requires additional processing. [56]

For the work herein using $\alpha$ particle emitting isotopes, a passive pixel sensor fabricated in-house at Carleton was used $[16,17]$. This allowed for full control of the device process flow and wafer level deposition of films for encapsulation and 
functionalization for biomolecule attachment.

For the work herein using $\beta$ particle emitting isotopes, a commercial CMOS APS from Canon was used. Early experiments showed that the sensor was sufficiently sensitive to $\beta$ particles, and the both the sensor and support electronics could be acquired at low cost. By comparison a MEDIPIX system would be more than an order of magnitude more expensive.

\subsection{Radioimmunotherapy}

Despite great effort, no universal cure for cancer exists, and many patients with metastasized diseases cannot be cured. Cancer treatment options vary based on the type of cancer and some treatments include surgery, external beam radiation therapy (EBRT), chemotherapy and radionuclide therapy. For localized diseases, surgery or EBRT remain the popular treatments. Chemotherapy and radionuclide therapy provide a treatment option in instances of disseminated and metastasizing tumors. Chemotherapy has been successful in treating a number of cancers, but its non-selective nature causes significant toxicity to healthy cells yielding unpleasant side effects and limiting the maximum dose that can be administered [69]. In order to limit these side effects, as well as increase the toxic dose administered to cancerous cells, a targeted approach is desirable.

Radionuclide therapy utilizes the DNA damaging properties of ionizing radiation, as described in Section 2.1.3, to kill cancerous cells. In targeted radionuclide therapy (TRT), or radioimmunotherapy (RIT), a radionuclide is attached to a cell targeting agent in order to promote delivery of the radioactive payload to the cancer site [70]. Both $\alpha$ and $\beta^{-}$particle emitting radioisotopes are of interest to create these radioimmunoconjugates (RIC). When using $\alpha$ particle emitting radioisotopes, the treatment is sometimes called targeted alpha therapy (TAT). To date, two $\beta^{-}$particle emitting 
RIT pharmaceuticals, have been approved for widespread use. One $\alpha$ emitting RIT pharmaceutical has been approved, while others are still undergoing clinical trials [30].

The following subsections will introduce various cell targeting agents, radioisotope payloads, as well as expand on the current state of pharmaceutical development.

\subsubsection{Targeting Agents and Methods}

Monoclonal antibodies (mAbs) appear to be the most popular targeting agent, but aptamers are also of interest. Proposed benefits of aptamers include the ease at which they can be synthesized as well as increased tumor penetrating ability due to their small size [71]. Both aptamers and mAbs are intended to target the cancer cell directly. An alternate method is pre-targeting where the tumor tissue is labelled with an identifying molecule, and it is this identifying molecule which is targeted. In [72] mAbs conjugated with a biotin derivative were injected to target the cancerous cell and later, recombinant streptavidin (rSAv) functionalized with a radionuclide was injected. This alternative method is similar to a military technique of "painting" targets with a laser for identification and to assist in bomb guidance.

\subsubsection{Radioisotope Payloads}

A number of radioisotopes are under investigation in RIT. The list below, paraphrased from [30], provides the characteristics of suitable isotopes while Table 2.2 provides a subset of isotopes of interest in current and future RIT pharmaceuticals [73].

1. Unless desired for imaging or dosimetry purposes, the isotope should not be a $\gamma$ particle emitter. In general $\alpha, \beta^{-}$, and Auger electron radiation is preferred.

2. The half life of the radionuclide should be 1.5 - 3 times longer than required for the RIC to achieve peak uptake. Half lives on the order of 1 - 14 days are 
considered optimal. A shorter half live can be beneficial if the tumor is readily accessible, but must be long enough to allow for RIC formation.

3. The chemical properties of the radionuclide should be such that a stable RIC is expected and little is accumulated in healthy tissue and organs.

4. The toxicity of radionuclide progeny should be considered.

5. A reliable, low cost, and pure source of the radionuclide should be available.

\begin{tabular}{cccc} 
Radionuclide & Emission type & Average Energy [keV] & Half Life \\
\hline \hline${ }^{211} \mathrm{At}$ & $\alpha$ & 5868 & $7.2 \mathrm{~h}$ \\
${ }^{212} \mathrm{Bi}$ & $\alpha$ & 6051 & $61 \mathrm{~min}$ \\
${ }^{213} \mathrm{Bi}$ & $\alpha$ & 5870 & $46 \mathrm{~min}$ \\
${ }^{225} \mathrm{Ac}$ & $\alpha$ & $5915^{*}$ & $10.0 \mathrm{~d}$ \\
\hline${ }^{32} \mathrm{P}$ & $\beta^{-}$ & 695 & $14.3 \mathrm{~d}$ \\
${ }^{67} \mathrm{Cu}$ & $\beta^{-}$ & 141 & $2.6 \mathrm{~d}$ \\
${ }^{77} \mathrm{As}$ & $\beta^{-}$ & 226 & $1.6 \mathrm{~d}$ \\
${ }^{90} \mathrm{Y}$ & $\beta^{-}$ & 935 & $2.7 \mathrm{~d}$ \\
${ }^{131} \mathrm{I}$ & $\beta^{-}$ & 181 & $8.0 \mathrm{~d}$ \\
${ }^{177} \mathrm{Lu}$ & $\beta^{-}$ & 140 & $6.7 \mathrm{~d}$ \\
${ }^{186} \mathrm{Re}$ & $\beta^{-}$ & 329 & 3.7 \\
${ }^{188} \mathrm{Re}$ & $\beta^{-}$ & 795 & $0.7 \mathrm{~d}$ \\
\hline
\end{tabular}

Table 2.2: Non-exhaustive list of isotopes of interest for RIT pharmaceuticals. $\left(^{*}\right)$ indicates a weighted average.

The characteristics of a particular radionuclide may be suited to different types of cancers. High-energy $\beta^{-}$emitters may travel several millimeters in organic tissue and are best suited to large bulky tumors. The non-linear path that $\beta^{-}$particles follow leads to an effect known as crossfire were a grouping of diseased cells are struck from a single emission. Because of their increased range and multi-cell targeting, damage to 


\begin{tabular}{ccc} 
Property & $\alpha$ & $\beta^{-}$ \\
\hline \hline Energy $[\mathrm{MeV}]$ & $4-9$ & $0.1-2.5$ \\
Range in tissue $[\mu \mathrm{m}]$ & $30-110$ & $150-13500$ \\
Range $[$ cell diameters] & $1-10$ & $10-1000$ \\
LET $[\mathrm{keV} / \mu \mathrm{m}]$ & $80-130$ & $0.2-0.7$ \\
Effect & DSB & SSB, crossfire
\end{tabular}

Table 2.3: Summary of the properties of $\alpha$ and $\beta^{-}$emitting radioisotopes for RIT. healthy tissue needs to be considered when using $\beta^{-}$therapy. Low-energy $\beta^{-}$emitters can be used, but since a large number of $\beta^{-}$interactions may be required to kill the cell, preference is given to using $\alpha$ emitting radioisotopes. Small and disseminated, single tumor cells are best treated with $\alpha$ emitting radioisotopes where the bulk of the energy is deposited locally. It is possible for a single $\alpha$ particle to cause a lethal number of DSB to a cell. Table 2.3 summarizes the properties of $\alpha$ and $\beta^{-}$emitting radioisotopes for RIT.

In order to produce a RIC these radioisotopes will have to conjugated with their targeting agent. For some $\beta^{-}$emitters, ${ }^{32} \mathrm{P}$ for example, this can be done by substitution of an atom in the targeting agent itself. In the case of the much larger $\alpha$ emitters, the conjugation is often achieved with a chelator. In [74] several chelators were tried with ${ }^{213} \mathrm{Bi}$ and DOTA was found to have the best in-vivo stability. It is important to note however, that although the chelator can retain the radionuclide prior to decay, the progeny will be liberated from the chelator from the recoil energy upon $\alpha$ emission. It is thus necessary to consider how the progeny will be cleared from the body, or internalized, after treatment.

Of the $\alpha$ emitters listed in Table 2.2 , actinium-225 $\left({ }^{225} \mathrm{Ac}\right)$ is particularly interesting. Actinium-225 will emit four $\alpha$ particles in relatively short succession as seen in Figure 2.12. This property makes it very appealing for RIT pharmaceuticals, and 


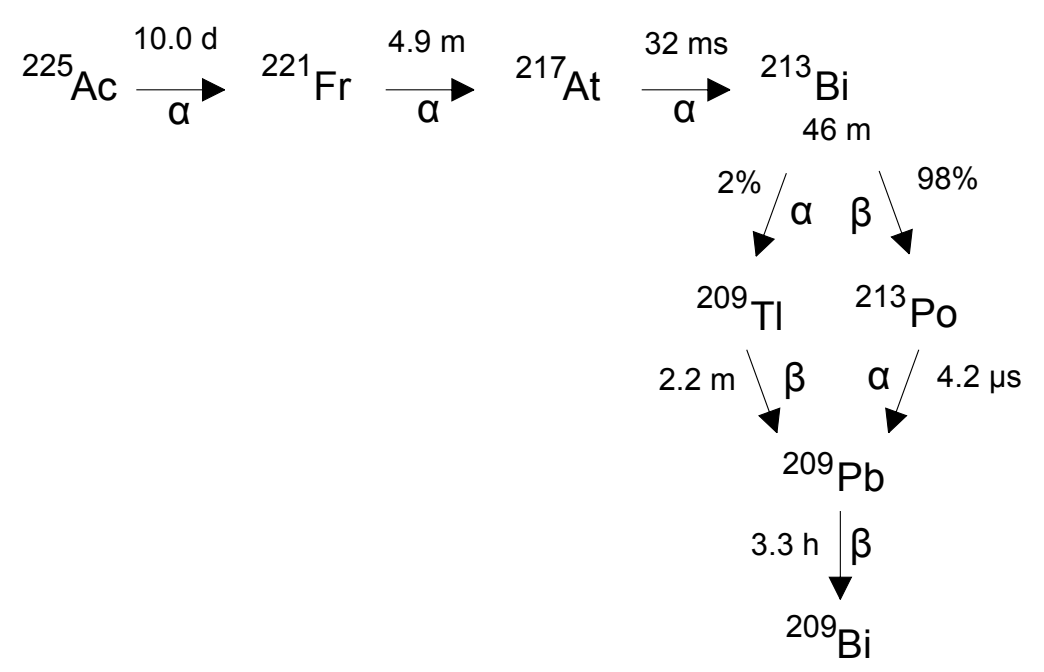

Figure 2.12: Actinium-225 decay chain.

a number of groups are looking to harness its potential [30,75-77].

\subsubsection{Current State of Pharmaceutical Development}

At the time of writing, only two RIT pharmaceuticals have been approved for widespread use: Zevalin ${ }^{\circledR}$ and Bexxar ${ }^{\circledR}$. Zevalin ${ }^{\circledR}$ was first approved in 2002 for the treatment of non-Hodgkins lymphoma and is a ${ }^{90} \mathrm{Y}$ labelled anti-CD20 monoclonal antibody. Bexxar ${ }^{\circledR}$ followed Zevalin ${ }^{\circledR}$ in 2003 and is labelled with ${ }^{131} \mathrm{I}$.

In 1996, the first human trials of a ${ }^{213} \mathrm{Bi}$ RIC began [78]. To date, the most advanced TAT pharmaceutical is Alpharadin ${ }^{\circledR}$, recently re-branded as Xofigo ${ }^{\circledR}$, which has been approved for the treatment of prostate cancer and bone metastases. It is noteworthy that Xofigo ${ }^{\circledR}$ makes use of ${ }^{223}$ Ra which, like ${ }^{225} \mathrm{Ac}$, emits a cascade of four $\alpha$ particles in relatively short succession. 


\subsection{Chemistry}

This project relies on the successful localization of radiolabelled biomolecules on a substrate for detection. In one case the biomolecules will be labelled prior to localization, while in another the labelling will happen in-situ on the surface of the detector. Localization chemistries are dependent on the substrate surface. Silane and gold-thiol chemistries are common for functionalizing glass and gold surfaces, respectively, for the attachment of biomolecules. [79-81]

Labelling chemistries for various biomolecules such as DNA and proteins are also well established and companies such as Invitrogen provide pre-made labelling kits. By functionalizing a surface with a molecule which exhibits a specific affinity reaction with a radiolabelled biomolecule, the radiolabelled molecule can be localized and detected. Examples of specific affinity reactions include the streptavidin-biotin reaction [72], antibody-antigen binding [82], the hybridization of complementary singlestranded DNA sequences [1] and aptamer chemistries [71,83].

An aptamer is a customized oligomer which is engineered to undergo a specific affinity reaction with a particular biomolecule. By preparing the aptamer with either an amine or thiol linker, the aptamer can be attached to desired locations on a glass or gold substrate respectively. For example, the amine modified aptamer sequence 5'$\mathrm{NH}_{2}-\left(\mathrm{CH}_{2}\right)_{6}$-ACC TGG GGG AGT ATT GCG GAG GAA GGT-3' exhibits a specific affinity reaction for adenosine triphosphate (ATP). The Chemistry Department at Carleton University is heavily involved in aptamer research.

\subsubsection{Gold-Thiol Chemistry}

A thiol is an organosulfur compound consisting of a carbon bonded sulfhydryl group (-SH). The carbon bond may be to an alkane, alkene or other carbon containing molecule. A strong bond can be formed between gold and the sulfhydryl group. This 
allows carbon containing molecules to be tethered to gold surfaces and is generally referred to as gold-thiol chemistry. Gold-thiol chemistry is very well researched and is a tool commonly used by chemists. Utilizing this chemistry DNA, RNA and other biomolecules can be attached to gold surfaces [84].

\subsubsection{Silane Chemistry}

SIMS-NRC has developed a chemistry for attaching amine-modified biomolecules to glass surfaces. The first step in their process is to prepare the glass surface with hydroxyl (-OH) groups. An organofunctional alkoxysilane molecule is then introduced. The hydroxyl groups then attack and displace the alkoxy groups on the silane forming a covalent $\mathrm{Si}-\mathrm{O}-\mathrm{Si}$ bond tethering the remaining molecule to the glass surface. N-hydroxysuccinimide, known as NHS, is then used to prepare the immobilized molecule for bonding with an amine. An amine terminated molecule, such as an amine terminated aptamer, can attach at the silane site immobilizing it on the sensor surface [85].

\subsection{Similar Work}

Detection of DNA radiolabelled with $\beta^{-}$emitting isotopes has previously been carried out with a CCD image sensor in [86] and proposed with the GaAs version of the MEDIPIX hybrid pixel sensor in [10]. No results with a CMOS image sensor have been found. The bulk of the research using CMOS technology appears to focus on autoradiography $[10,11,47,49,51,87-89]$.

In autoradiography, spatial resolution is a primary concern. This leads researchers to use low-energy $\beta^{-}$emitting isotopes like tritium and specialized back-thinned detectors. This concern is relaxed for quantitative analysis of attached $\beta^{-}$radiolabelled biomolecules or $\beta^{-}$RIT pharmaceuticals. 
Also in autoradiography there is no attachment of the radiolabelled biomolecules to a solid substrate. The radiolabels are contained within a tissue sample placed on or near the detector surface. In the work presented herein experiments with radiolabelled biomolecules will be localized on a solid substrate by chemical processing, or directly on the detector itself.

Cabello's works $[47,88,89]$ presented some Monte Carlo simulations which attempted to analyze the effects of pixel size and epitaxial layer thickness in CMOS and CCD imagers for autoradiography. This analysis is similar to the work presented in this dissertation. However, the intent of the simulations performed here is to investigate a CMOS radiation sensor specifically for the quantitative analysis of localized radiolabels. 


\section{Chapter 3}

\section{Simulation}

In order to make quantitative measurements of radiolabelled molecules it will be necessary to have a thorough understanding of the radiation detection system. As is often the case in modelling both radiation physics and semiconductor device physics, developing analytic solutions may not be possible, and numerical methods are required for modelling purposes. This chapter presents the use of two numerical tools, GEANT4, and Sentaurus, towards better characterization of the radiation detection system used in experiments involving $\beta^{-}$particle emitting isotopes in Chapter 4 and demonstrates that a dedicated detector need not be designed and that a commercial sensor will be sufficient for the task.

\subsection{Geant4 Introduction}

GEANT4 is an object-oriented $\mathrm{C}++$ toolkit written by the European Organization for Nuclear Research (CERN) to simulate the passage of particles through matter. It includes a wide array of radiation physics models, valid over a wide energy range, coupled with a Monte Carlo simulation framework, allowing a broad spectrum of programs to be written. Commonly used for high energy physics (HEP) simulations, the introduction of models extending the utility of the toolkit to tens of eV allow 
lower energy applications to be simulated. Models which have been extended to lowenergy applications include Livermore, Penelope, Geant4-DNA, and MicroElec. A short description of each model follows.

- Livermore models are valid for electrons and $\gamma$ rays and have a recommended energy range of $250 \mathrm{eV}$ to $100 \mathrm{GeV}$, but in principle they can be used down to $10 \mathrm{eV}$ with reduced accuracy below $100 \mathrm{eV}$. Validation of these models was performed in [90]. With respect to electrons, the low-energy limits for ionization and Bremsstrahlung are $100 \mathrm{eV}$ and $10 \mathrm{eV}$, respectively.

- Penelope models have a recommended energy range of $100 \mathrm{eV}$ to $1 \mathrm{GeV}$ and are valid for electrons, positrons and photons [91]. With respect to electrons, the low-energy limits for ionization and Bremsstrahlung are both $100 \mathrm{eV}$.

- Geant4-DNA models were developed for modelling biological damage induced by ionizing radiation in DNA [92]. A number of models for processes involving electrons, protons and some heavy ions are included, but the models are not valid for use in silicon.

- MicroElec models were developed specifically for the simulation of electrons, protons, and heavy ions in silicon. For electrons, elastic and inelastic scattering are modelled with low-energy limits of $5 \mathrm{eV}$ and $16.7 \mathrm{eV}$, respectively [93], and with high-energy limits of $100 \mathrm{MeV}$. Validation work on these models can be found in [94].

In order to simulate using GEANT4 it is necessary to create an object-oriented $\mathrm{C}++$ program that utilizes classes made available in the GEANT4 source distribution. At the very least, it is necessary to implement a RunManager, DetectorConstruction, PhysicsList, and a PrimaryEventGenerator. Intuitively, the PrimaryEventGenerator is responsible for generating the primary particle being simulated and for setting the 


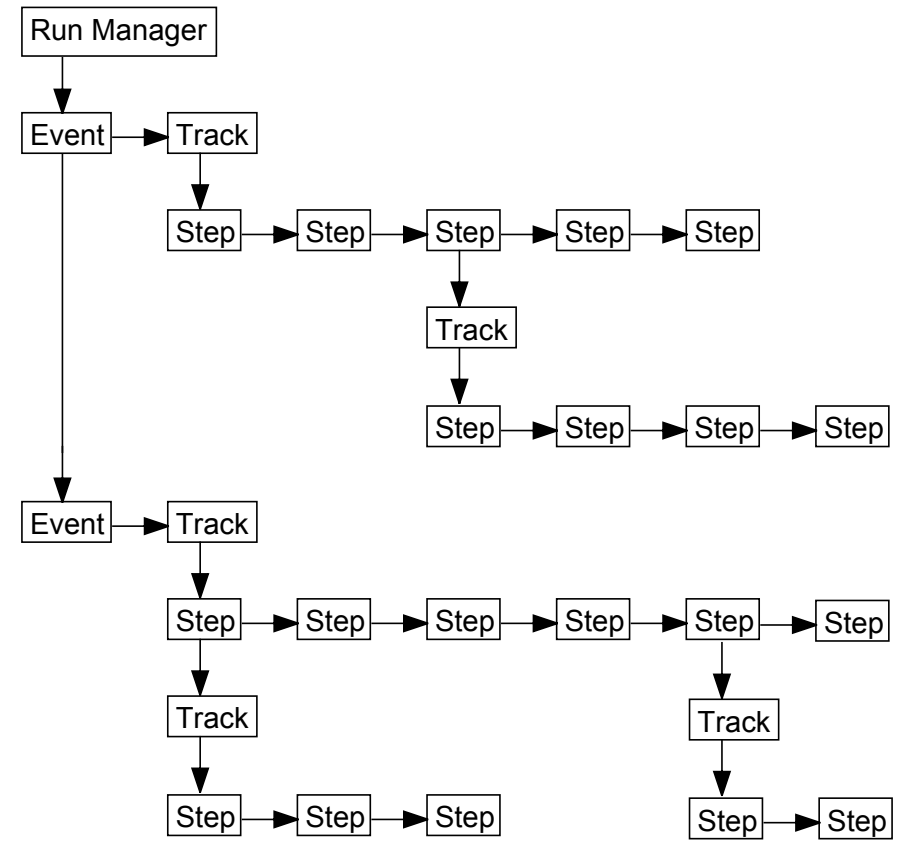

Figure 3.1: GEANT4 simulation flow.

particle type, energy, and direction. It is possible to utilize the GeneralParticleSource class (GPS) to generate complex particle sources within the PrimaryEventGenerator. The PhysicsList provides a list of all physical processes that each type of particle being simulated experiences, while the DetectorConstruction defines the detector's shape, size and material. Lastly, the RunManager is responsible for initializing, and managing, all the simulations being run. These four elements are the base requirement for any GEANT4 program, however, a number of other classes are available to assist in data analysis, particle tracking, visualization, and more. Figure 3.1 presents the general flow of a typical simulation. The RunManager generates a particle to begin an event, the event starts a track which generates a step at each interaction. If the interaction generates a secondary particle, that particle is entered into a queue, and this queue will be processed once the parent track has concluded. Tracks will continue to step until the energy of the particle is below a set value. 
This particle tracking ability allows the charge and energy deposition within the detector, as well as the effect of the sensor's packaging on the deflection of the incident ionizing radiation, to be observed.

\subsection{Geant4 Simulations}

GEANT4 simulations were carried out with software compiled using GeAnT4.10.00P01. Using the GPS class, a variety of source distributions and locations were simulated. Depending on the phenomena and materials being simulated, different models were used. In the case of observing charge deposition in a silicon slab, the MicroElec models were used, while the Livermore models were used when tracking particles in multi-material structures. Simulations were performed in order to validate the software, to observe the energy and charge deposited for various isotopes, and to observe the effect of the detector's packaging.

\subsubsection{Software Validation}

In order to assess the software's ability to produce accurate simulation data, a set of simulations were run comparing simulated results with data from the National Institute for Standards and Technology (NIST) databases for $\alpha$ and $\beta^{-}$radiation.

To begin validating the software, a slab of silicon was simulated using the MicroElec models. Inside the slab was a rectangular block of silicon $6.4 \mu \mathrm{m}$ square and $3 \mu \mathrm{m}$ deep. This volume was chosen as it matches the dimensions of the active volume of the Canon 705S CMOS MAPS used for $\beta^{-}$detection in Chapter 4. The charged secondaries generated in this block by $5 \mathrm{MeV} \beta^{-}$particles emitted $10 \mu \mathrm{m}$ from the top of the block surface were simulated. Charged secondaries were generated down to the low-energy limit of the model. The $10 \mu \mathrm{m}$ spacer is used to represent the MAPS dielectric stack, encapsulation, colour filters and lenses. Figure 3.2 presents 


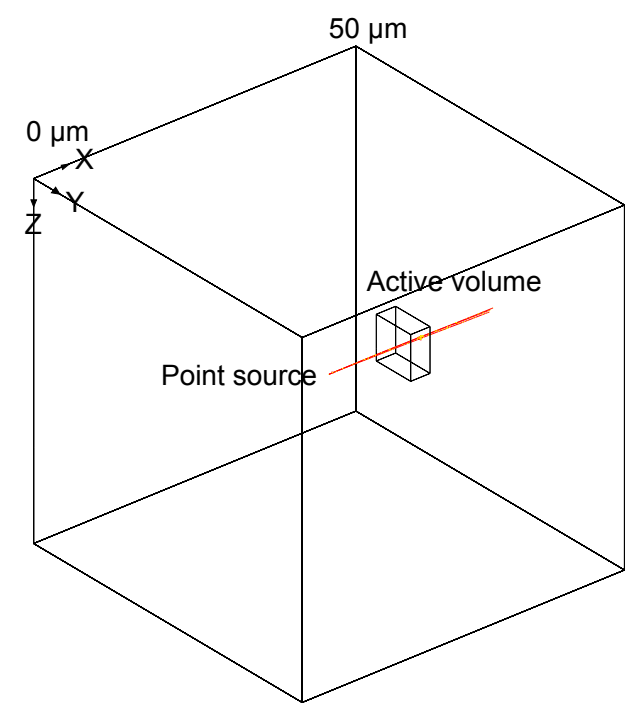

Figure 3.2: GEANT4 software validation structure showing three $5 \mathrm{MeV} \beta^{-}$particles.

the simulation showing three $5 \mathrm{MeV} \beta^{-}$particles, while Figure 3.3 shows the charged secondaries created from 20,000 $\beta^{-}$particles. Excluding rare $\delta$ rays, the tracks of the charged secondaries were on average fractions of a micron. The track length of charged secondaries from these simulations are presented in Figure 3.4.

Simulation times using the MicroElec models are substantially longer compared to using the Livermore models. Depending on the energy of the incident particle, a single event can take tens of minutes to simulate using MicroElec, whereas the Livermore models take seconds using a single core of a $3.1 \mathrm{GHz}$ Intel Xeon E5-2687W processor. In order to reduce the time required for these simulations the dimensions of the silicon slab were set to $50 \mu \mathrm{m}$ cubed, and $\beta^{-}$leaving this volume were no longer tracked. The particle source is located in the center of the cube and emits mono-energetic $\beta^{-}$particles normally incident towards the active volume.

Figure 3.5 presents data from NIST showing the stopping power of $\beta^{-}$particles in silicon as a function of energy [95]. For $\beta^{-}$particles within the 1 - $10 \mathrm{MeV}$ range, the rate of energy deposition, and hence charge generation, in silicon is relatively constant. This consistency has led to the figures of merit used for charge deposition by 


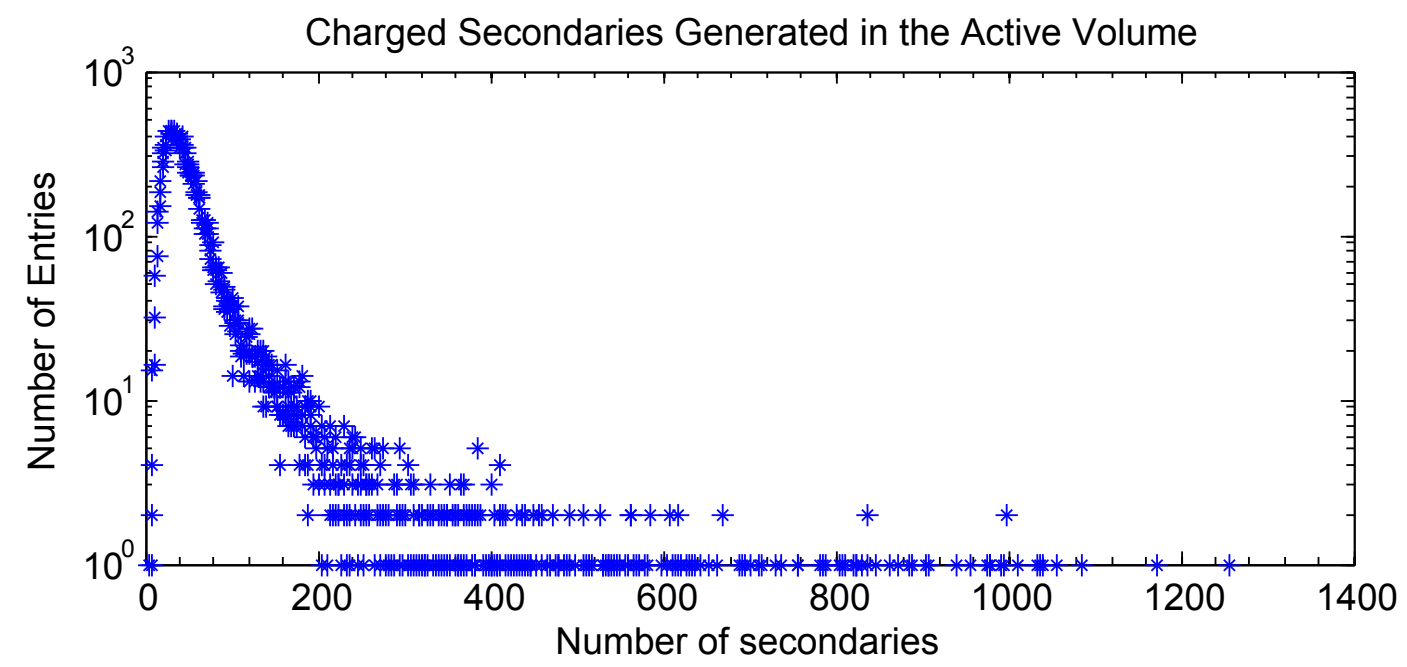

Figure 3.3: Histogram of charged secondaries generated by twenty thousand $5 \mathrm{MeV}$ $\beta^{-}$particles passing through a $6.4 \mu \mathrm{m}$ square and $3 \mu \mathrm{m}$ deep block of silicon using the GEANT4 MicroElec models.

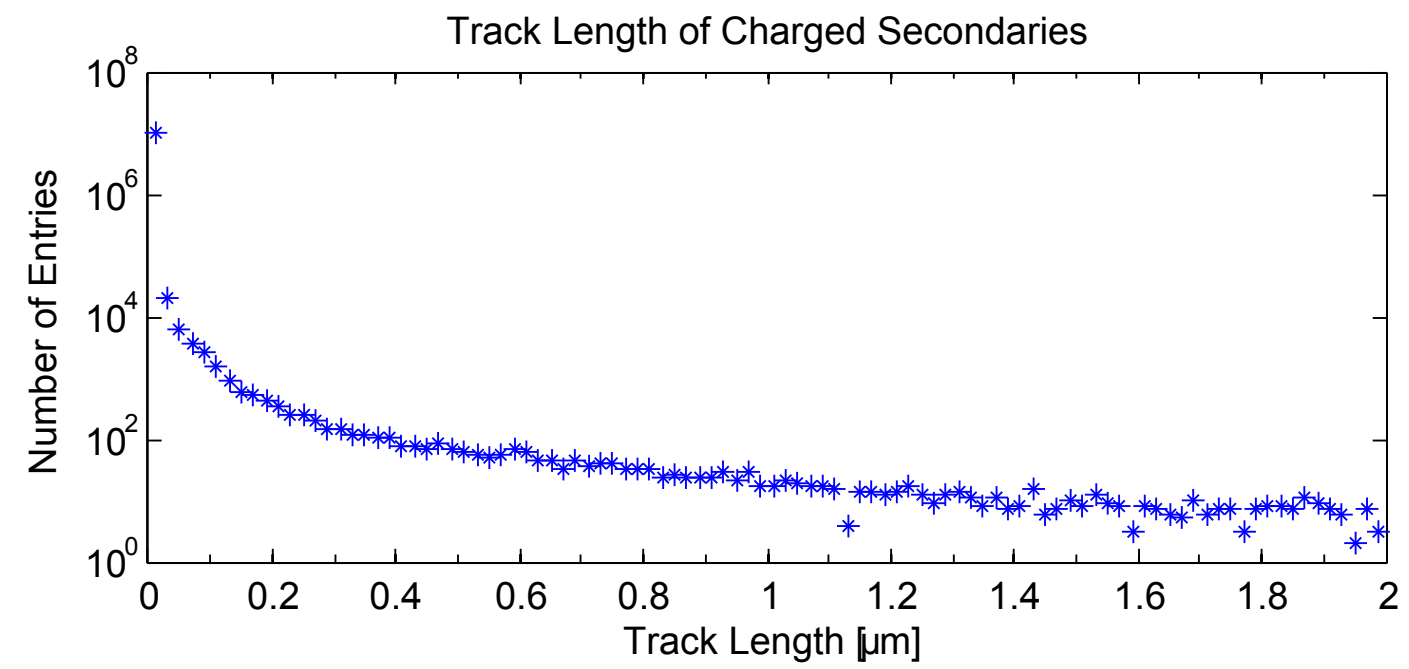

Figure 3.4: Histogram showing the track length of charged secondaries generated by twenty thousand $5 \mathrm{MeV} \beta^{-}$particles in a block of silicon using the GEANT4 MicroElec models. 


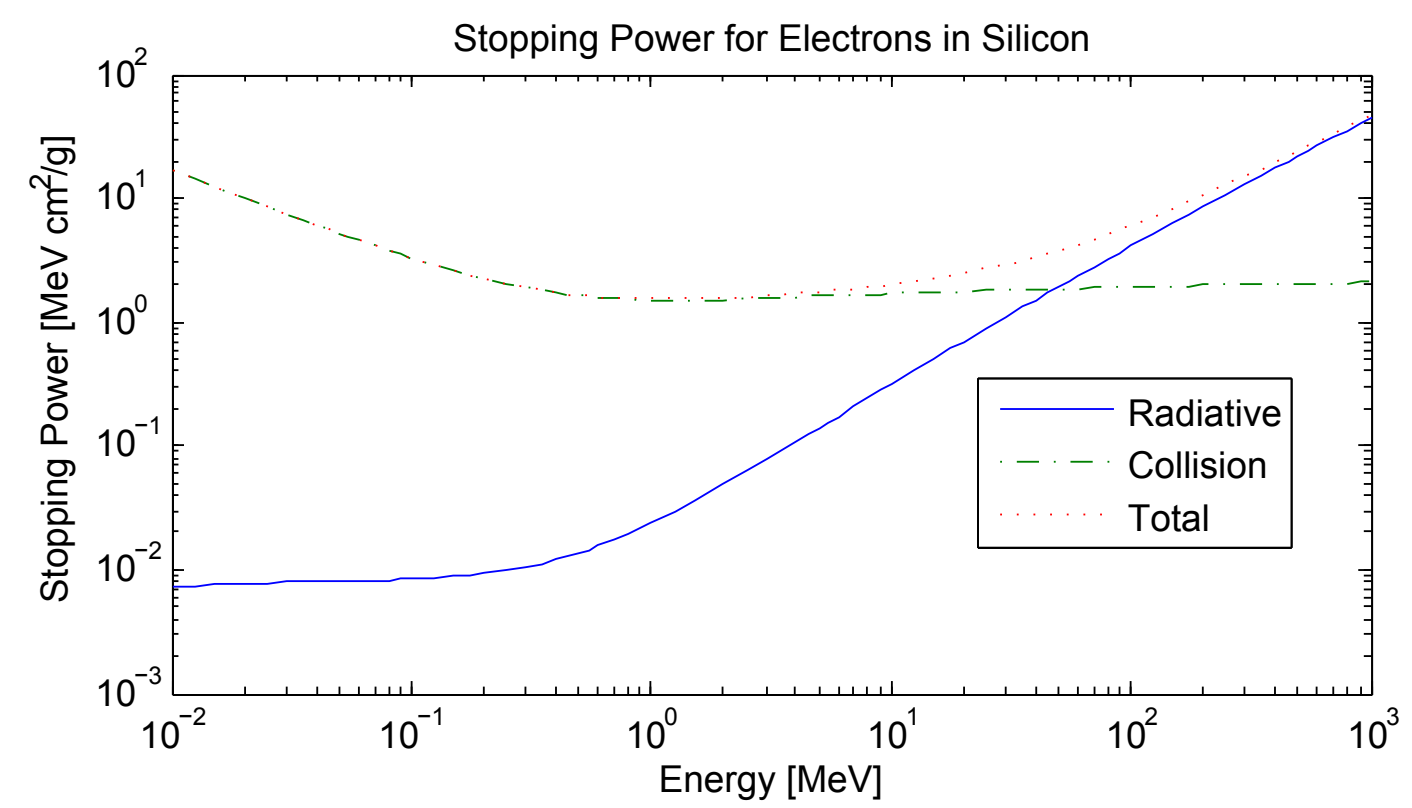

Figure 3.5: Data from the NIST ESTAR database showing the stopping power of $\beta^{-}$particles in silicon as a function of energy.

$\beta^{-}$particles interacting with silicon to be $76 \mathrm{e}-\mathrm{h} / \mu \mathrm{m}$ (most probable) and $108 \mathrm{e}-\mathrm{h} / \mu \mathrm{m}$ (average) [57]. Simulations here for mono-energetic $5 \mathrm{MeV} \beta^{-}$particles showed charge deposition in agreement with a Landau distribution with a mean charge generated per unit path length of $\frac{59 e-h}{3 \mu m}=19.7 e-h / \mu m$, nearly a factor of 6 less than expected. This discrepancy is likely due to the charged secondaries not being generated below $16.7 \mathrm{eV}$. Some work has been done in [96] where particles were tracked down to $1.5 \mathrm{eV}$. Despite the charge generated per unit path length being lower than expected, the energy deposited per unit path length, or stopping power, is consistent with these figures of merit. Using the MicroElec model the average energy deposited per unit path length was simulated to be $426 \mathrm{eV} / \mu \mathrm{m}$ as seen in Figure 3.6. Using $3.6 \mathrm{eV} / \mathrm{e}-\mathrm{h}$ pair, $426 \mathrm{eV} / \mu \mathrm{m}$ would yield $118 \mathrm{e}-\mathrm{h} / \mu \mathrm{m}$, which compares favourably with 116 e$\mathrm{h} / \mu \mathrm{m}$ from the NIST data for $5 \mathrm{MeV} \beta^{-}$particles. Note that the particle source is located at $25 \mu \mathrm{m}$ and the stopping power is nearly constant with depth. The mean track length of charged secondaries was simulated to be $10 \mathrm{~nm}$. 


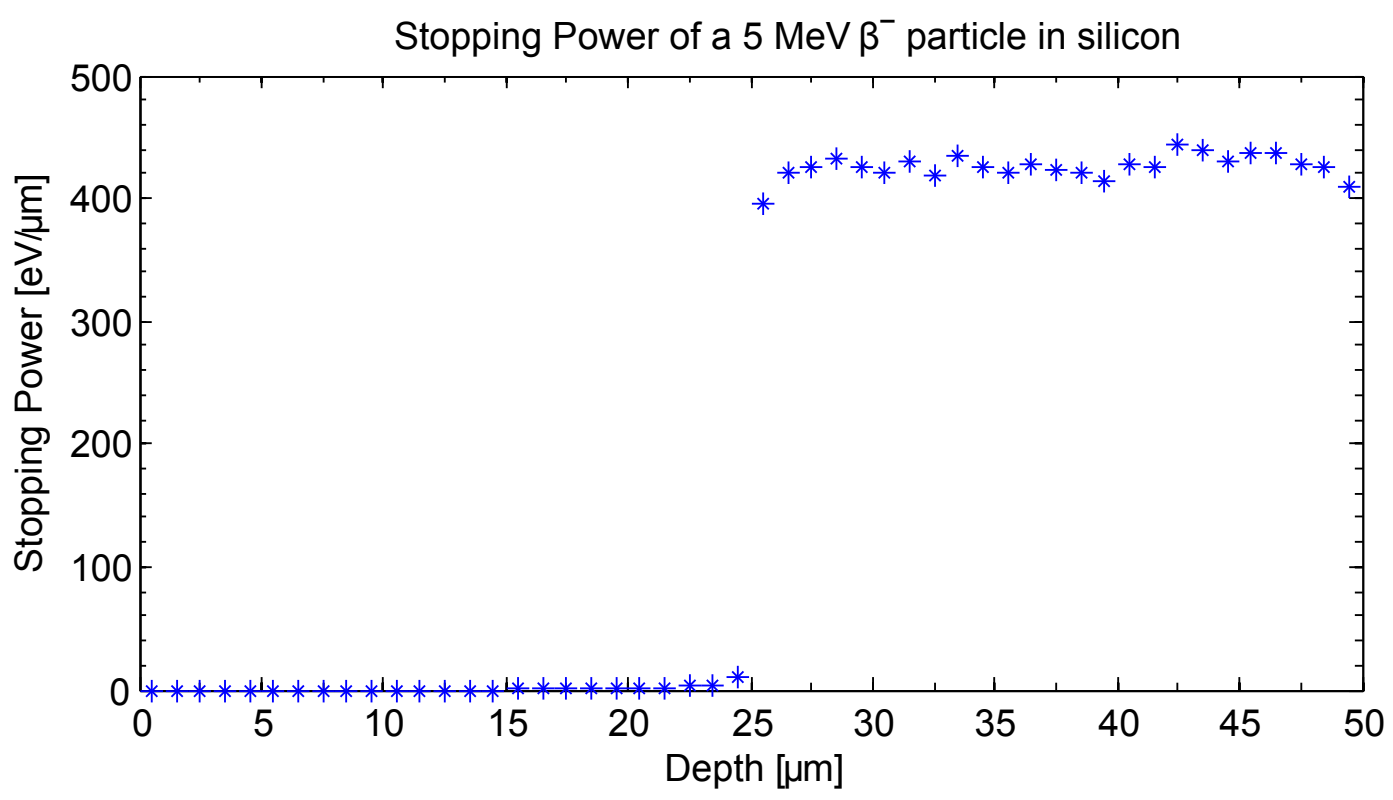

Figure 3.6: Histogram showing the energy deposited per unit length by twenty thousand $5 \mathrm{MeV} \beta^{-}$particles in a block of silicon using the GEANT4 MicroElec models.

As the MicroElec models are not valid for materials other than silicon, the Livermore models were used when simulating other materials. Using the Livermore models, a simulation was run with fifty thousand $5 \mathrm{MeV} \beta^{-}$particles normally incident on a $2 \mathrm{~cm}$ cubed silicon block for validation purposes. Figure 3.7 presents the simulation results. The average path length of the primary particle was simulated to be $12.7 \mathrm{~mm}$, in agreement with the NIST constantly slowing down approximation (CSDA) result of $12.8 \mathrm{~mm}$ [95]. The average depth the primary particle penetrated, known as the projected range, was simulated to be $6.75 \mathrm{~mm}$.

When simulating $\alpha$ particles, the Livermore models do not apply. As a final validation step, two hundred thousand $5.4 \mathrm{MeV} \alpha$ particles normally incident on a silicon block were simulated. These simulations were carried out using GEANT4's standard models while suppressing the generation of charged secondaries. Figure 3.8 presents the results showing the rate of energy deposition and the projected range. The energy deposition rate follows the expected shape of the Bragg curve, while 


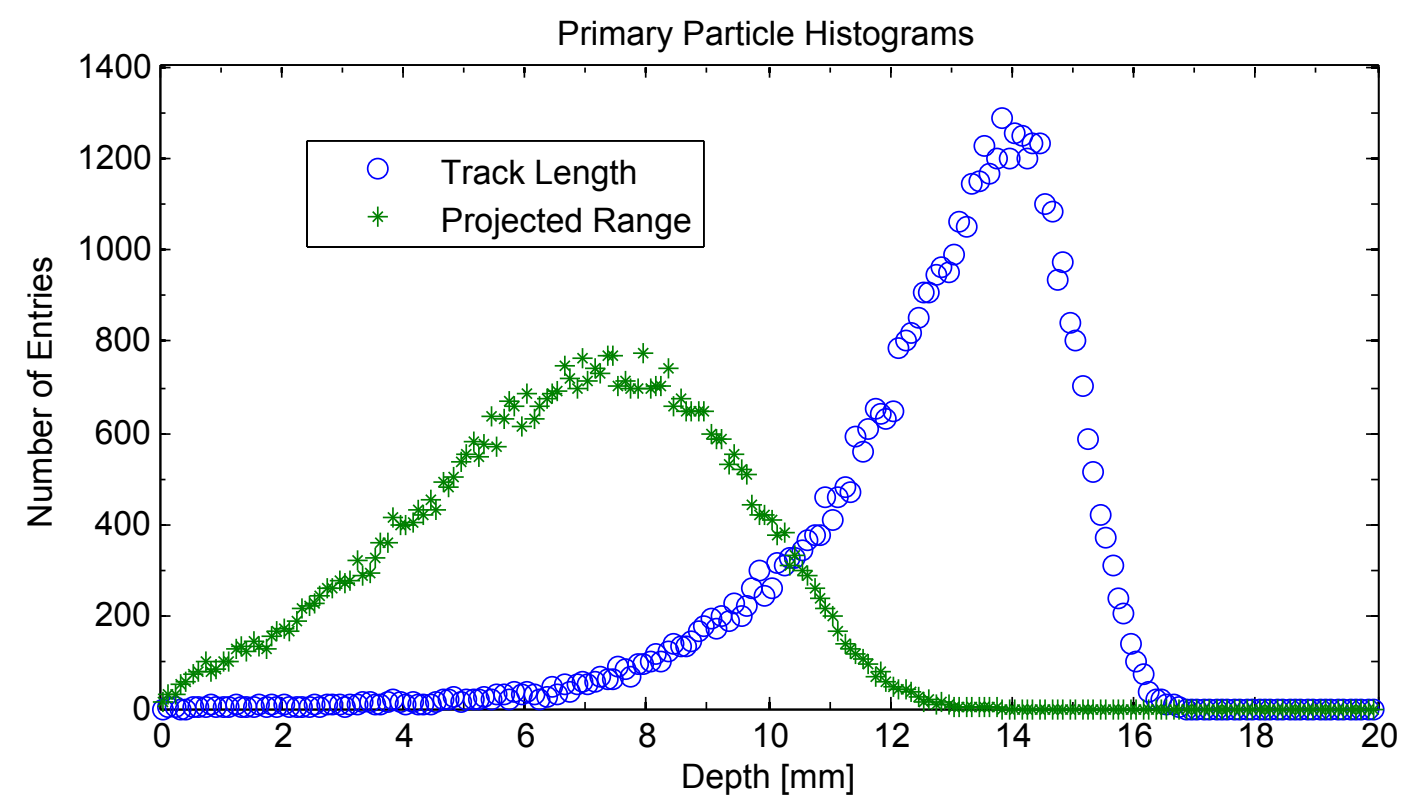

Figure 3.7: GEANT4 simulation of fifty thousand $5 \mathrm{MeV} \beta^{-}$particles normally incident on a $2 \mathrm{~cm}$ cubed block of silicon using the Livermore models.

the projected range was found to be $27 \mu \mathrm{m}$ in agreement with the NIST ASTAR database [97].

Having validated the software, charge and energy deposition in the detector were investigated.

\subsubsection{Charge \& Energy Deposition}

Emissions from the $\beta^{-}$emitting isotopes phosphorus-32 $\left({ }^{32} \mathrm{P}\right)$ and strontium-90 $\left({ }^{90} \mathrm{Sr}\right)$ have been used in sensor calibration and biological detection experiments. The emission spectrum from each source was coded into GEANT4 allowing the isotopes to be simulated using both the MicroElec and Livermore models. Both ${ }^{32} \mathrm{P}$ and ${ }^{90} \mathrm{Sr}$ source distributions were built using data from [98], and the data is presented in Figure 3.9.

Due to the inapplicability of the MicroElec models to materials other than silicon, these simulations were carried out with the particle source positioned as in Section 3.2.1, $10 \mu \mathrm{m}$ from the sensing volume of the pixel in a slab of silicon. When using the 


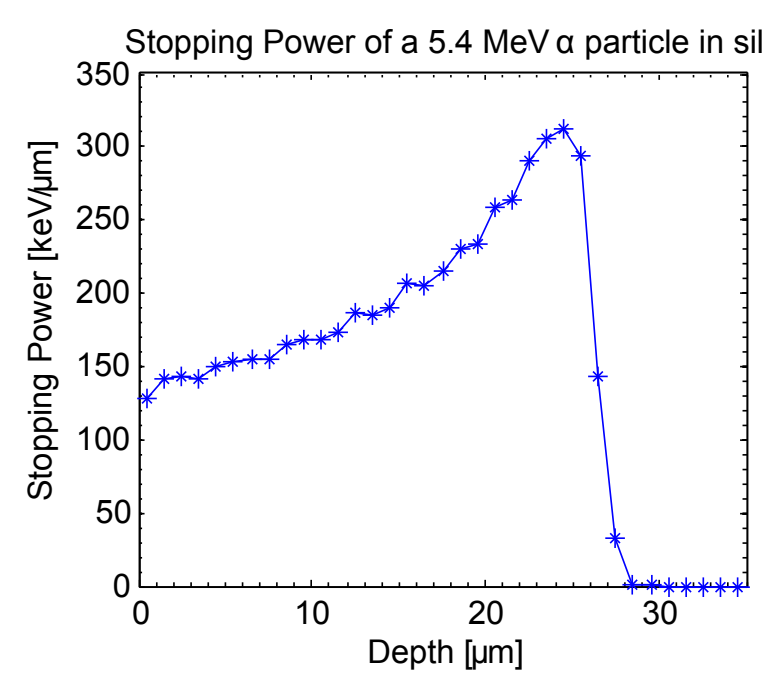

(a) Rate of energy deposition (stopping power)

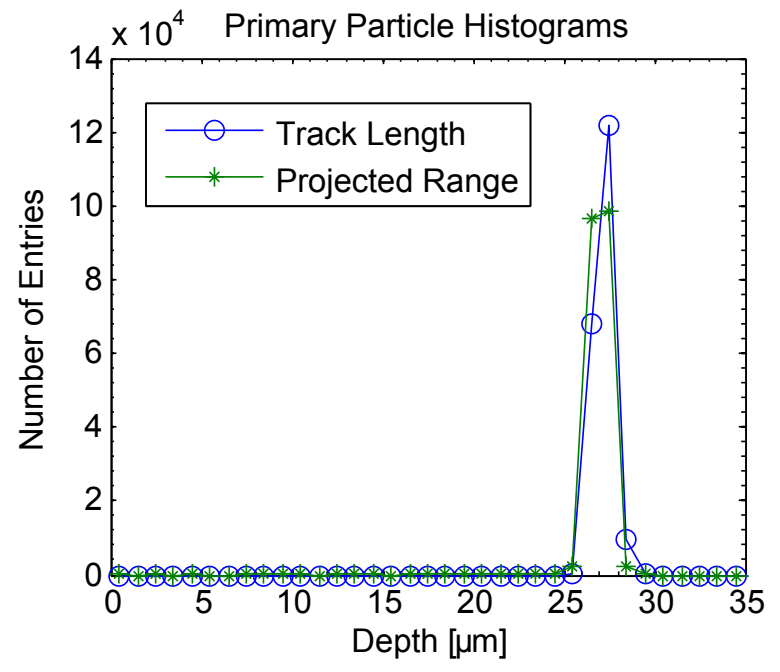

(b) Track length and projected range

Figure 3.8: GEANT4 simulation of two hundred thousand $5.4 \mathrm{MeV} \alpha$ particles normally incident on a block of silicon using the standard models.

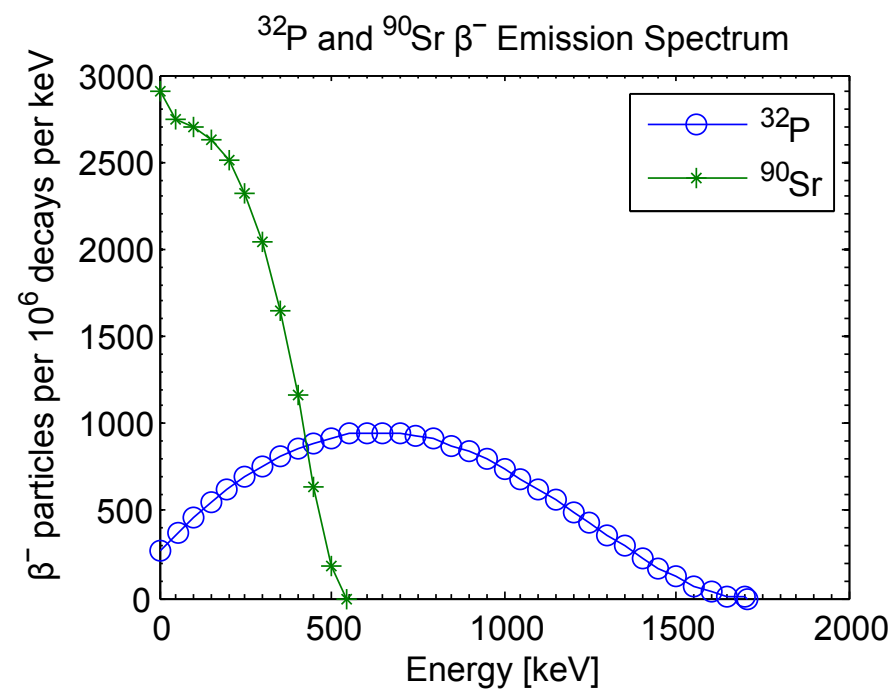

Figure 3.9: ${ }^{32} \mathrm{P}$ and ${ }^{90} \mathrm{Sr} \beta^{-}$emission spectrum. 


\begin{tabular}{cccc} 
Model & Cutoff & Average Charge Generated & Average Energy Deposited \\
\hline \hline MicroElec & $16.7 \mathrm{eV}$ & 60 electrons & $447 \mathrm{eV} / \mu \mathrm{m}$ \\
Livermore & $250 \mathrm{eV}$ & 0.36 electrons & $420 \mathrm{eV} / \mu \mathrm{m}$ \\
Livermore & $10 \mathrm{eV}$ & 10 electrons & $330 \mathrm{eV} / \mu \mathrm{m}$ \\
\hline
\end{tabular}

Table 3.1: Summary of GEANT $4{ }^{32} \mathrm{P}$ simulation results.

MicroElec models, the secondary generation cutoff energy was $16.7 \mathrm{eV}$ while when using the Livermore models lower cutoffs of $250 \mathrm{eV}$ and $10 \mathrm{eV}$ were simulated. Each simulation consists of 50,000 normally incident $\beta^{-}$particles.

Figure 3.10 presents charge and energy deposition simulation results for ${ }^{32} \mathrm{P}$, while Table 3.1 summarizes the results. From comparing the results it is clear that when simulating charge deposition, the MicroElec models provide better results than Livermore as the average charge generated was closer to expectation. However, both MicroElec and Livermore models offer good agreement in energy deposition over their validated range. At the theoretical limit of the Livermore models, the energy deposition simulated differs from expectation.

Also observed in these simulations is a variation in energy deposition as a function of depth. This dependence can be attributed to the distribution in emission energy of the radioisotope. Low-energy $\beta^{-}$particles will have less penetrating ability in an absorber due to both their low energy and increased stopping power. Hence, at shallow depths, more interactions will be observed, and these interactions will deposit more energy.

Figure 3.11 presents the charge and energy deposition simulation results with ${ }^{90} \mathrm{Sr}$, while Table 3.2 summarizes the results. Here the number of electrons generated is higher, and accordingly, so is the rate of energy deposition. ${ }^{90} \mathrm{Sr}$ has lower average and endpoint energies than ${ }^{32} \mathrm{P}$ leading to increased charge generation at shallower depths as compared to ${ }^{32} \mathrm{P}$. 


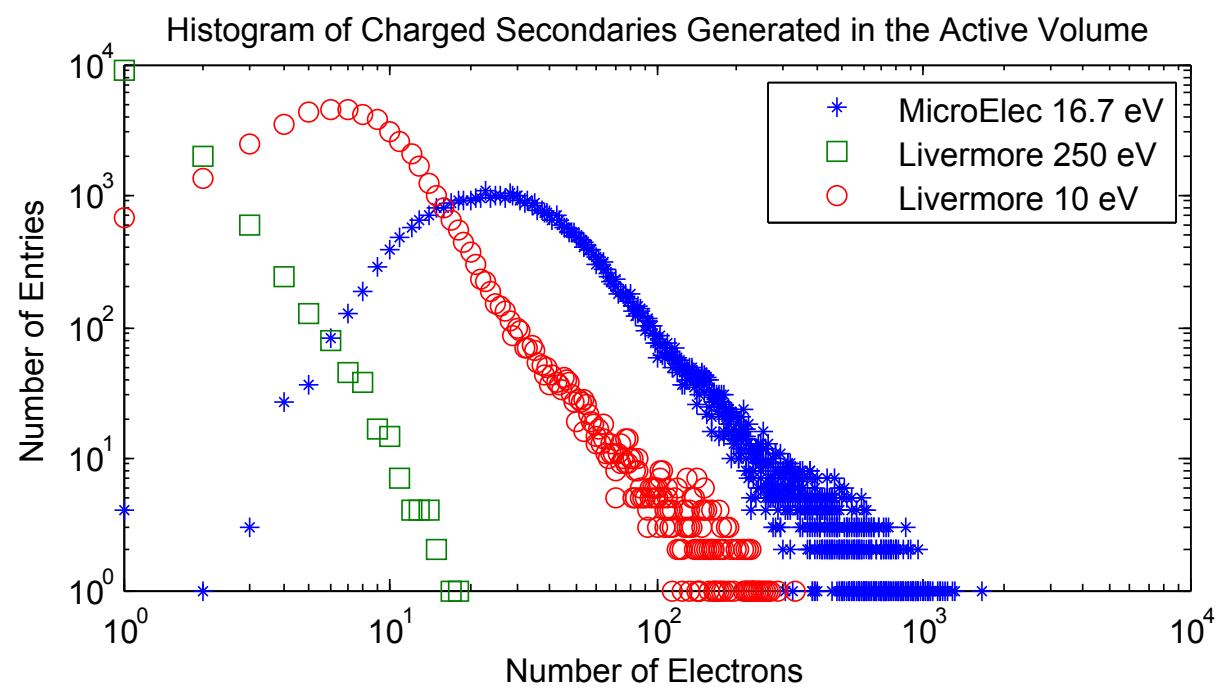

(a) Charge generation

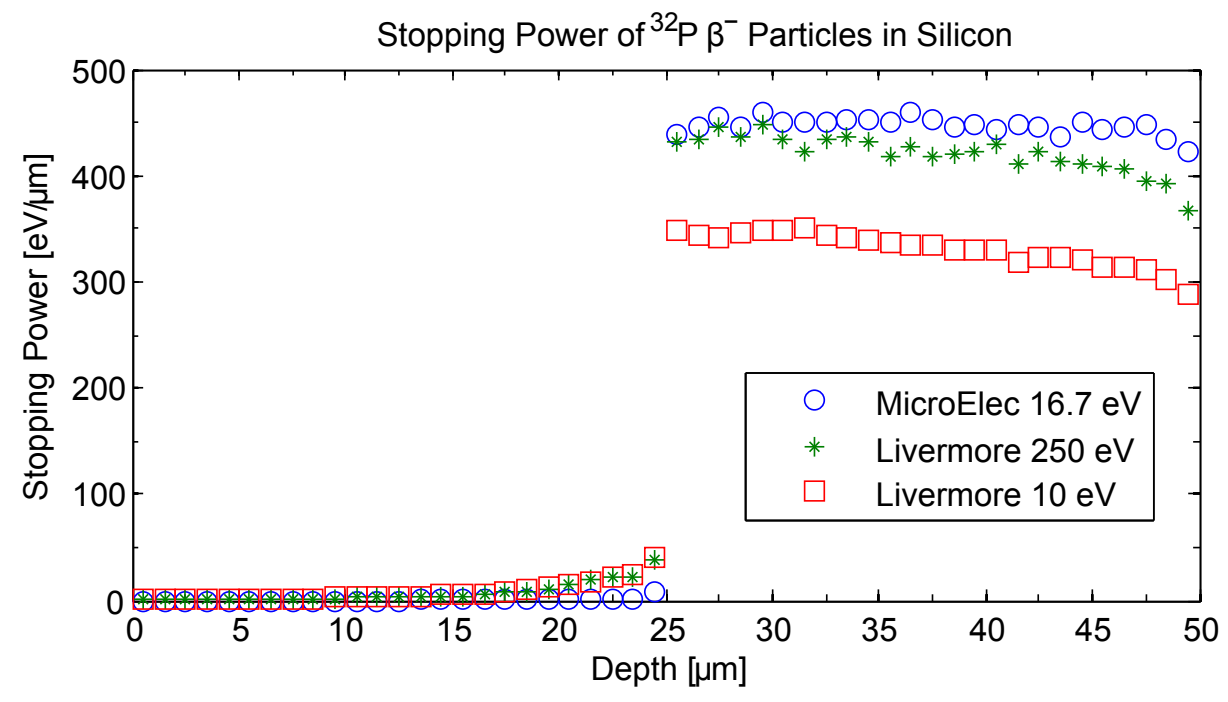

(b) Stopping power

Figure 3.10: GEANT4 charge and energy deposition simulation results for ${ }^{32} \mathrm{P}$ using the MicroElec model with a $16.7 \mathrm{eV}$ cutoff, as well as the Livermore model with $250 \mathrm{eV}$ and $10 \mathrm{eV}$ cut off for 50,000 normally incident $\beta^{-}$particles. 


\begin{tabular}{cccc} 
Model & Cutoff & Average Charge Generated & Average Energy Deposited \\
\hline \hline MicroElec & $16.7 \mathrm{eV}$ & 91 electrons & $756 \mathrm{eV} / \mu \mathrm{m}$ \\
Livermore & $250 \mathrm{eV}$ & 0.44 electrons & $829 \mathrm{eV} / \mu \mathrm{m}$ \\
Livermore & $10 \mathrm{eV}$ & 9 electrons & $604 \mathrm{eV} / \mu \mathrm{m}$ \\
\hline
\end{tabular}

Table 3.2: Summary of GEANT $4{ }^{90} \mathrm{Sr}$ simulation results.

Both MicroElec and Livermore models have provided comparable results in terms of energy deposition simulations, but differ substantially in their ability to simulate charged secondaries. The MicroElec models produced an average generated charge which was closer to expectation than Livermore even at the lowest theoretical tracking cutoff. Using the Livermore models with tracking cuts lower than $250 \mathrm{eV}$ produced more secondaries, but led to an appreciable change in stopping power. However, by using the Livermore models, in their validated range, and inferring the charge generation from energy deposition simulations, reasonable simulation results can be generated rapidly.

This section provided insight into two different GEANT4 models, and how two different isotopes would deposit charge and energy within a single pixel of a pixelated detector. The following section will investigate how the detector's packaging will influence particle detection.

\subsubsection{Packaging and Backscattering Effects}

The system level response of a radiation detector to $\beta^{-}$particles would be influenced by the detector's packaging as well as by scattering within the detector itself. For the experiments performed in Chapter 4 a Canon 705S CMOS MAPS was used to measure the emissions from ${ }^{32} \mathrm{P}$ and ${ }^{90} \mathrm{Sr}$. Simulations were performed using GEANT4 to calculate the number of $\beta^{-}$particles that would reach the sensor as well as pass through, and backscatter out of, the image sensor surface. Additionally, the energy 


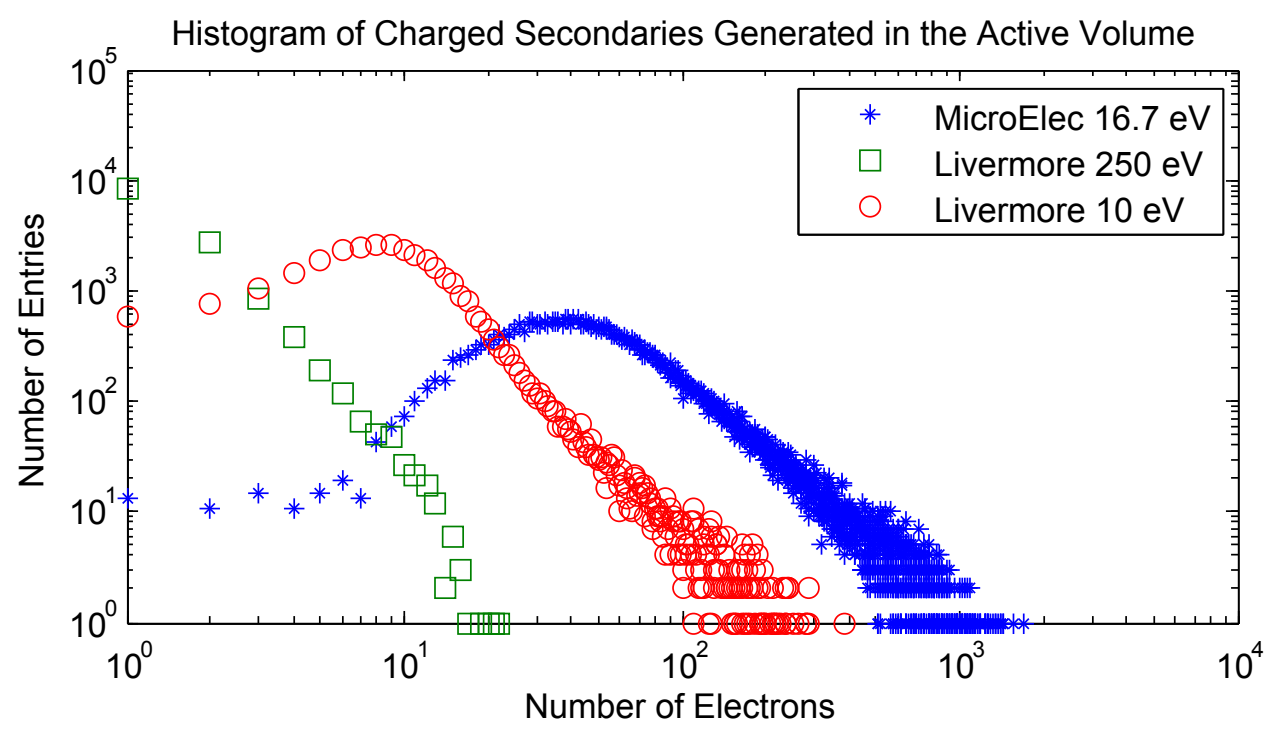

(a) Charge generation

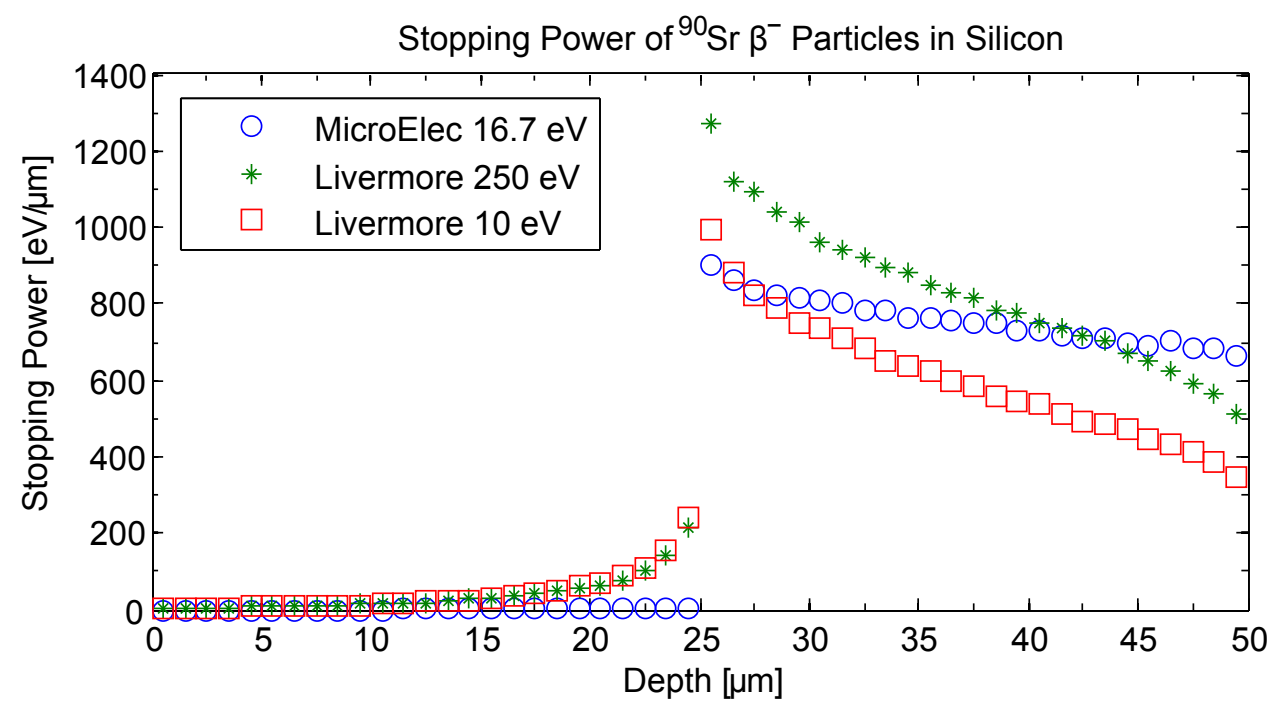

(b) Stopping power

Figure 3.11: GEANT4 charge and energy deposition simulation results for ${ }^{90} \mathrm{Sr}$ using the MicroElec model with a $16.7 \mathrm{eV}$ cutoff, as well as the Livermore model with $250 \mathrm{eV}$ and $10 \mathrm{eV}$ cut off for 50,000 normally incident $\beta^{-}$particles. 
deposited, and thus charge generated, in the active region of the MAPS can be observed, as well as the expected spatial resolution of the system. For these simulations the Livermore models were used with an energy cut-off of $250 \mathrm{eV}$. Livermore models were used due to their fast processing time and applicability in multi-material simulations.

The packaging for this particular sensor is shown in Figure 3.12. Measured from the topmost surface, there is a removable $2.51 \mathrm{~mm}$ infrared (IR) filter, $0.49 \mathrm{~mm}$ of coverglass, and a $0.75 \mathrm{~mm}$ air gap before reaching the surface. Experiments described in Chapter 4 were performed with radiolabelled samples functionalized on $100 \mu \mathrm{m}$ thick glass coverslips atop the sensor packaging, so this additional thickness must be considered. For simulation purposes the structure consists of a glass plate $0.59 \mathrm{~mm}$ thick (coverslip and coverglass thickness combined), an air gap of $0.75 \mathrm{~mm}$, and an oversized $2.50 \mathrm{~mm}$ silicon substrate. The top $10 \mu \mathrm{m}$ of the silicon substrate was considered to approximate the micro-lens array, Bayer layer, and encapsulation of the sensor. Particles passing through, and back out of, the $10 \mu \mathrm{m}$ deep interface layer were counted separately. A point source, centered on the outermost layer of the glass plate, emits $\beta^{-}$particles isotropically. Above the point source, a very low density gas, G4_Galactic, is used to ensure that that $\beta^{-}$particles emitted away from the packaging rapidly exit the simulation.

Simulation results showing 10 events generated from a $900 \mathrm{keV} \beta^{-}$source are shown in Figure 3.13. For ease of display, Figure 3.13 shows a sensor surface area of $5 \mathrm{~mm} \times 5 \mathrm{~mm}$. Note the absorption in the glass, nonlinear paths, and backscattering. Backscattering and absorption will reduce the sensitivity of the system, while the nonlinear path can result in multiple clusters of pixels being activated across the sensor. Figure 3.13 also demonstrates the reduction in spatial resolution attributed to scattering in the glass and lateral travel in the air gap.

Figure 3.14 presents the results after simulating $50 \times 10^{6}$ emissions from a ${ }^{32} \mathrm{P}$ 


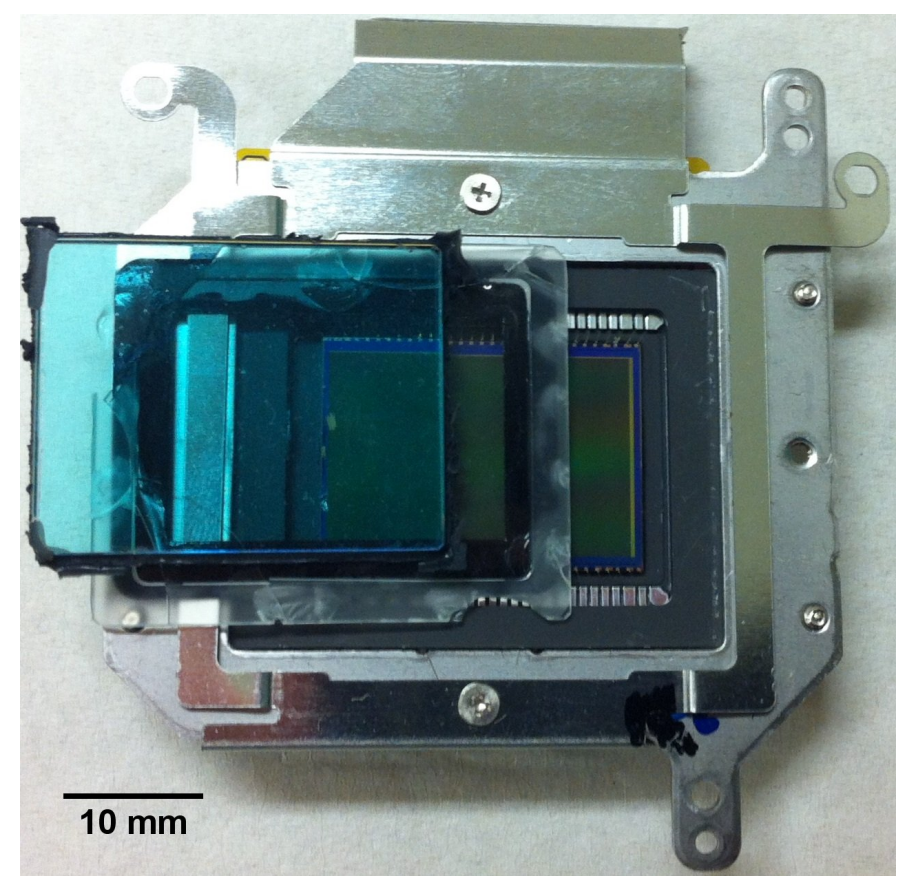

Figure 3.12: Canon 705S CMOS MAPS packaging.

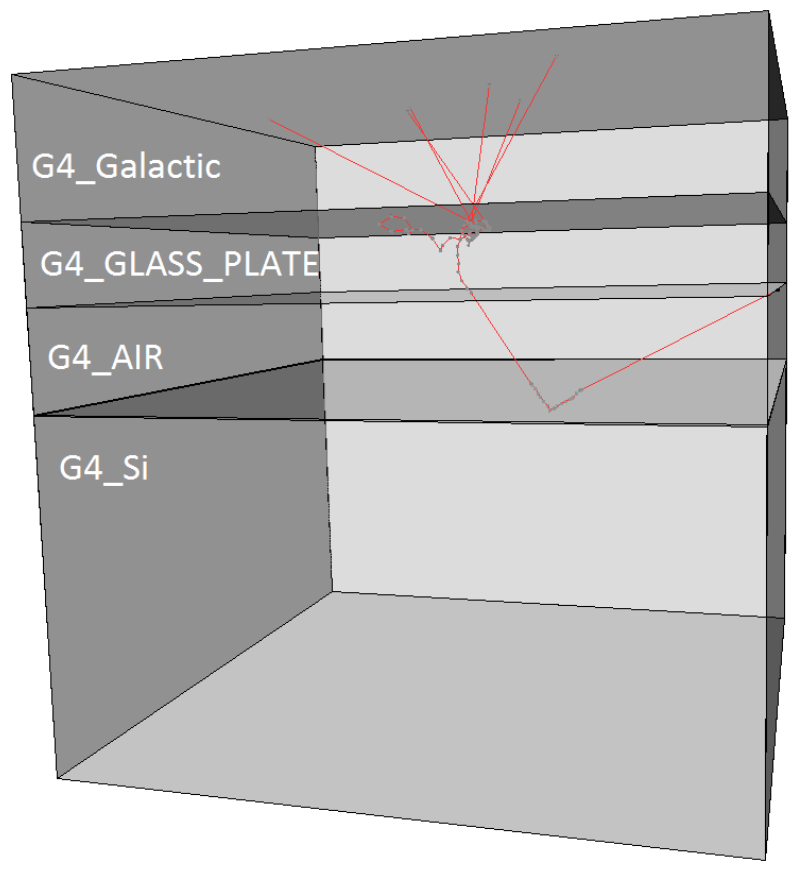

Figure 3.13: Canon 705S CMOS MAPS simulation structure showing ten $900 \mathrm{keV}$ $\beta^{-}$particles emitted isotropically from a point source. A $5 \times 5 \mathrm{~mm}^{2}$ surface area is shown. 
point source. Figure 3.14(a) presents a 2D histogram showing the total number of events passing through the 705S active layer surface. Figure 3.14(b) shows a slice through the peak of Figure 3.14(a) and distinguishes between the particles entering, and backscattering out of, the active layer surface. The spatial resolution of this system will be defined as the full-width half-maximum (FWHM) of Figure 3.14(b), and it was found to be $1.8 \mathrm{~mm}$. Using the number of events recorded in each histogram, the fraction of $\beta^{-}$particles passing into the sensitive layer was $15 \%$, while the number scattering out was $4 \%$. The combination of these two values produces the fraction of possible detection events, which was found to be $19 \%$. Figure 3.15 presents the rate of energy deposition inside the 705S packaging and sensor. Inside the $3 \mu \mathrm{m}$ thick sensitive layer the average rate of energy deposition was simulated to be $149 \mathrm{eV} / \mu \mathrm{m}$. These simulations were repeated for ${ }^{90} \mathrm{Sr}$, without the $100 \mu \mathrm{m}$ thick glass coverslip present, and the results are summarized in Table 3.3. It should be noted that the results with ${ }^{90} \mathrm{Sr}$ show a stopping power in the active layer too small, on average, to generate a measurable signal. However, ${ }^{90} \mathrm{Sr}$ 's daughter Yttrium-90 $\left({ }^{90} \mathrm{Y}\right)$, also decays through $\beta^{-}$emission with a half life of 64 hours and an endpoint energy of $2.28 \mathrm{MeV}$. When using an ${ }^{90} \mathrm{Sr}$ source for measurements it is more likely that the ${ }^{90} \mathrm{Y}$ emissions, with an endpoint energy higher than ${ }^{32} \mathrm{P}$, are the source of any measurable signal. It is also noteworthy that the stopping power simulated using the Livermore models is initially higher, and decreases faster, than the MicroElec models. This would result in the Livermore models predicting less charge generation in the active volume than MicroElec when simulating lower energy $\beta^{-}$particles.

Having simulated the system level response of the $705 \mathrm{~S}$ sensor to $\beta^{-}$particles emitted from ${ }^{32} \mathrm{P}$ and ${ }^{90} \mathrm{Sr}$, a semiconductor simulation tool was used to investigate the expected electrical response. Details are provided in the following section. 
Total Number of Events Passing Through the Active Layer Surface

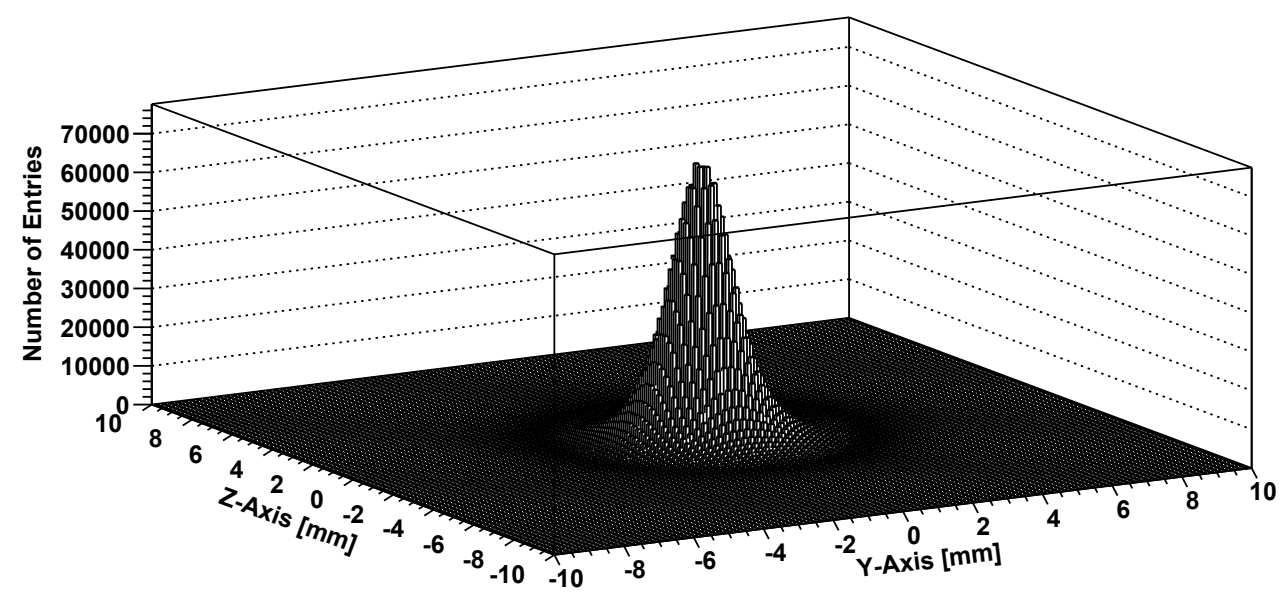

(a) $2 \mathrm{D}$ histogram of the total number of $\beta^{-}$particles passing into, and out of, the active layer surface

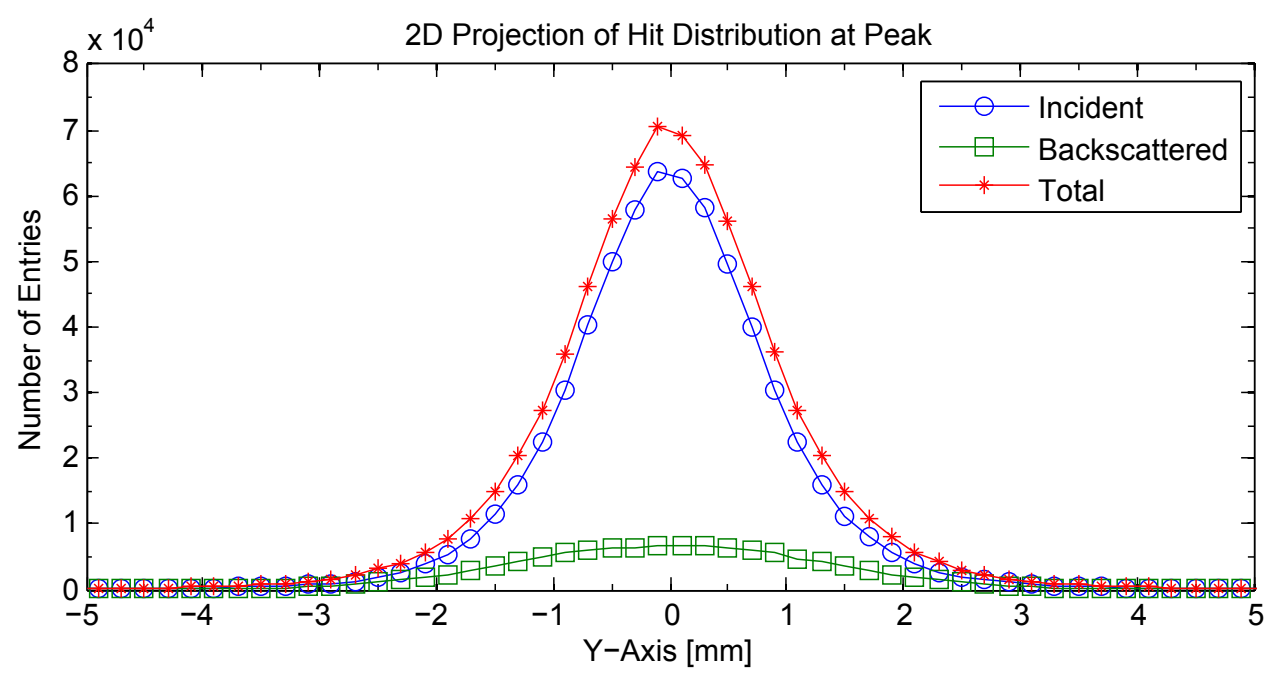

(b) Projection showing the distribution of incident, backscattered, and total number of $\beta$ particles passing through the active layer surface

Figure 3.14: 2D histogram, and projections, of ${ }^{32} \mathrm{P} \beta^{-}$particles entering and exiting the surface of a Canon 705S image sensor with $50 \times 10^{6}$ events simulated using GEANT4 with Livermore models. 


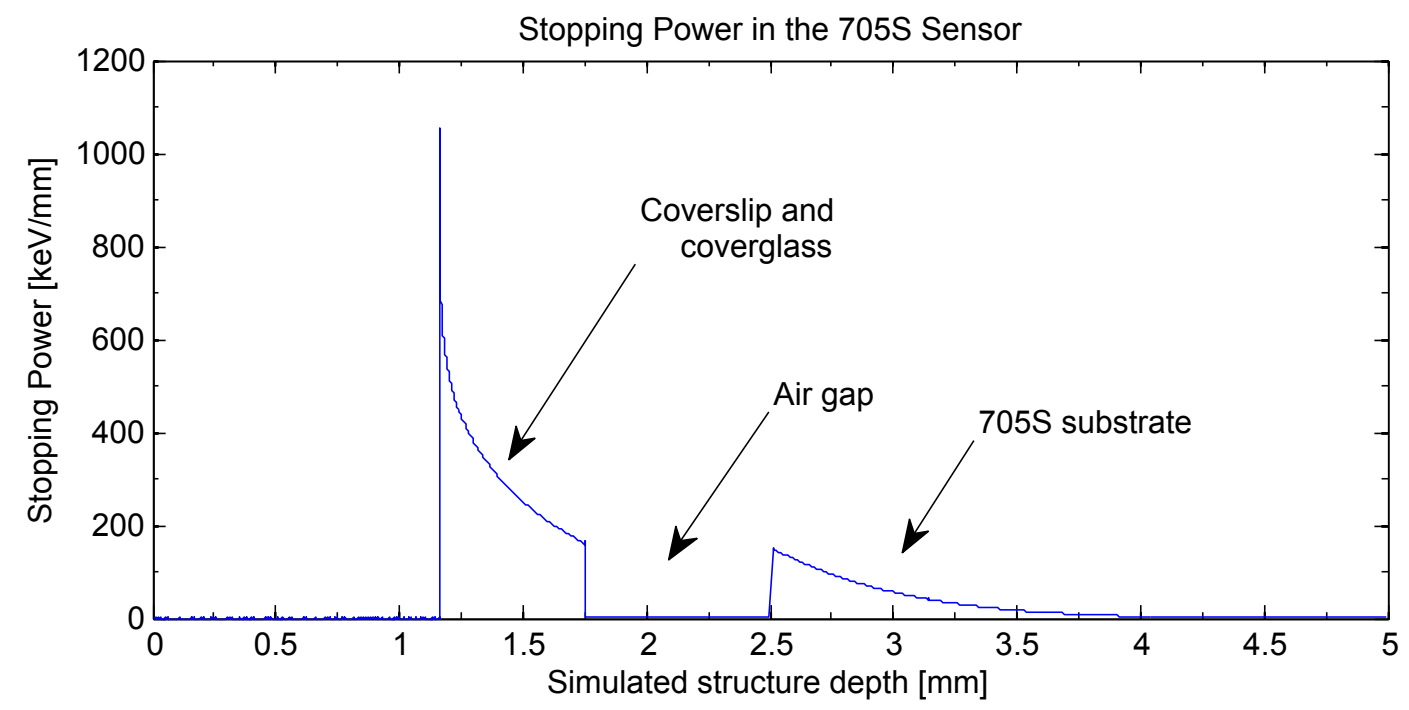

Figure 3.15: Stopping power in the Canon 705S CMOS MAPS packaging and sensor from $50 \times 10^{6}$ events from an isotropic ${ }^{32} \mathrm{P}$ point source.

\begin{tabular}{ccc} 
Parameter & ${ }^{32} \mathrm{P}$ & ${ }^{90} \mathrm{Sr}$ \\
\hline \hline \% reaching the sensitive layer & 15 & 0.3 \\
\% scattering out of the sensitive layer & 4 & 0.06 \\
FWHM $[\mathrm{mm}]$ & 1.8 & 1.1 \\
$\frac{\Delta E}{\Delta x}$ in the active volume $[\mathrm{eV} / \mu \mathrm{m}]$ & 149 & 3.5 \\
\hline
\end{tabular}

Table 3.3: System level GEAnT4 simulation results of the Canon 705S sensor and packaging for both ${ }^{32} \mathrm{P}$ and ${ }^{90} \mathrm{Sr}$ simulated with $50 \times 10^{6}$ events. Note that the glass coverslip was not present in the simulation with ${ }^{90} \mathrm{Sr}$. 


\subsection{Sentaurus Introduction}

Sentaurus, offered by Synopsys, represents a suite of semiconductor process and device simulation tools. Sentaurus Device is the toolset's device modelling tool which includes some ability to simulate the effects of ionizing radiation. Transient columnar ionization paths can be introduced to simulate the passage of an ionizing particle. These columnar distributions would be expected for the passage of an $\alpha$ particle, but with a suitable LET, they can be used to approximate ionization from a $\beta^{-}$particle.

For relatively short distances, a linear column of ionization can be a suitable approximation for the passage of a $\beta^{-}$particle. Figure 2.3 presented a $\beta^{-}$particle passing through a silicon slab where each point was the final location of an ionized electron. Over distances of several microns, the trajectory was somewhat linear. As has been described before, typically 80 e-h $/ \mu \mathrm{m}$ could be expected for $\beta^{-}$particles between 1-10 MeV, which correlates to $12.8 \times 10^{-6} \mathrm{pC} / \mu \mathrm{m}$. Developing an interface between Geant4 and Sentaurus was considered but deemed impractical due to the computational requirements to run a device simulation for each GEANT $4 \beta^{-}$particle trajectory. Instead, a typical ionization event was used.

Running a Sentaurus device simulation involves defining and meshing a structure using either Sentaurus Processes, or Sentaurus Structure Editor, and feeding that structure into Sentaurus Device. By parameterizing either the Sentaurus Process, or Sentaurus Structure Editor input files, an array of simulations can be carried out automatically using the Sentaurus Workbench graphical user interface (GUI). With the structures defined, Sentaurus Device can run transient and other simulations. Much like Geant4, the Sentaurus Device input files must include the list of device physics to be included, as well as information on any incident ionizing radiation to be simulated. In the following section, Sentaurus Version J-2014.09 was used to investigate the charge collection efficiency of the Canon 705S CMOS MAPS. Appendix A 


\begin{tabular}{r|l} 
Parameter & Value \\
\hline \hline Die size & $22.2 \times 14.8 \mathrm{~mm}$ \\
Substrate* $^{*}$ & N-type \\
Epitaxial layer thickness* & $5.5 \mu \mathrm{m}$ \\
Substrate doping* & $4 \times 10^{15} \mathrm{~cm}^{-3}$ \\
Epitaxial layer doping* & $4 \times 10^{15} \mathrm{~cm}^{-3}$ \\
Pixel type* $^{*}$ & Buried photocathode in P-well \\
P-well profile* & Doubly implanted \\
P-well depth* & $3 \mu \mathrm{m}$ \\
Pixel size $^{*}$ & $6.4 \mu \mathrm{m} \mathrm{square}$ \\
Buried photocathode size* & $3.2 \times 2.8 \mu \mathrm{m}$ \\
Dieletric/Metal \& Bayer/Lens stack thicknesses* & $3.9 \mu \mathrm{m} \& 3.7 \mu \mathrm{m}$ \\
\hline
\end{tabular}

Table 3.4: Canon 705S process details. $(*)$ denotes information from Chipworks [99].

contains the Sentaurus input files used in this work.

\subsection{Sentaurus Simulations}

The detector used in the experiments with $\beta^{-}$particles, to be described in Chapter 4 , was a Canon 705S CMOS MAPS. It is a proprietary sensor for which no public data sheet is available. Chipworks, a reverse engineering firm in Ottawa, Ontario, Canada generously provided access to a report which provided some details on the sensor's fabrication. This information was used to build 2D and 3D models for simulation using Sentaurus Device. Table 3.4 provides key process details [99]. Despite the lack of a publicly available datasheet for this sensor, Dr. Roger N. Clark maintains a database of image sensor performance specifications which includes the 705S sensor [100]. Table 3.5 lists some of the specifications from his database. 


\begin{tabular}{r|l} 
Parameter & Value \\
\hline \hline Full well capacity & 43000 electrons \\
Read noise & 3.7 electrons at ISO 1600 \\
Unity gain ISO & 1040 \\
RAW image size & $2314 \times 3474$ \\
ADC & 12 bit \\
Gain @ ISO1600 & 0.6 electrons/ADU
\end{tabular}

Table 3.5: Canon 705S sensor specifications [100].

All simulations were carried out using the same physical processes being modelled without any changes to default material parameters. Carrier mobility was simulated with doping dependencies, high field saturation and transverse field effects enabled. Bandgap narrowing was simulated using the Oldslotboom model, and generation/recombination models included Shockley-Read-Hall, Auger and avalanche breakdown [41]. Photodiode contacts were biased through $1 \mathrm{~T} \Omega$ resistors to approximate a floating junction. Both the amount of charge collected by, and the potential change of the photodiode, were automatically extracted using the Inspect tool. Mesh refinements were made in the photodiode region and along the path of the ionizing radiation. The mesh was made incrementally more fine until no appreciable change was seen in the simulation results. Unless indicated otherwise, the charge deposition rate of the ionizing radiation was $80 \mathrm{e}-\mathrm{h} / \mu \mathrm{m}$ with a Gaussian distribution around the particle track with a $0.1 \mu \mathrm{m}$ standard deviation. Despite simulations being carried out with photodiodes biased at both $3.3 \mathrm{~V}$ and $5 \mathrm{~V}$, only results with $3.3 \mathrm{~V}$ are shown. A small increase in charge collection is seen with $5 \mathrm{~V}$ biased photodiodes due to their larger depletion regions, but the results showed that diffusion, more than drift, was the principle means of charge collection in agreement with [47]. 


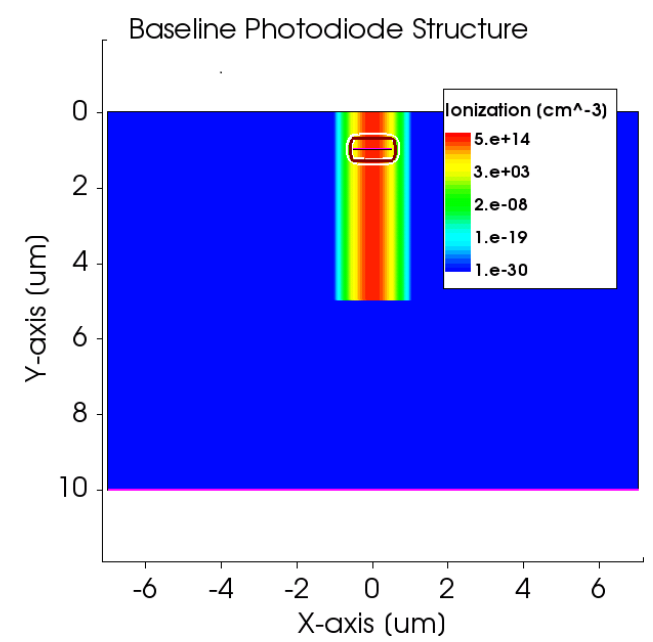

(a) 2D simulation structure showing the depletion region (bordered in white), and ionization from a normally incident $\beta^{-}$ particle penetrating $5 \mu \mathrm{m}$

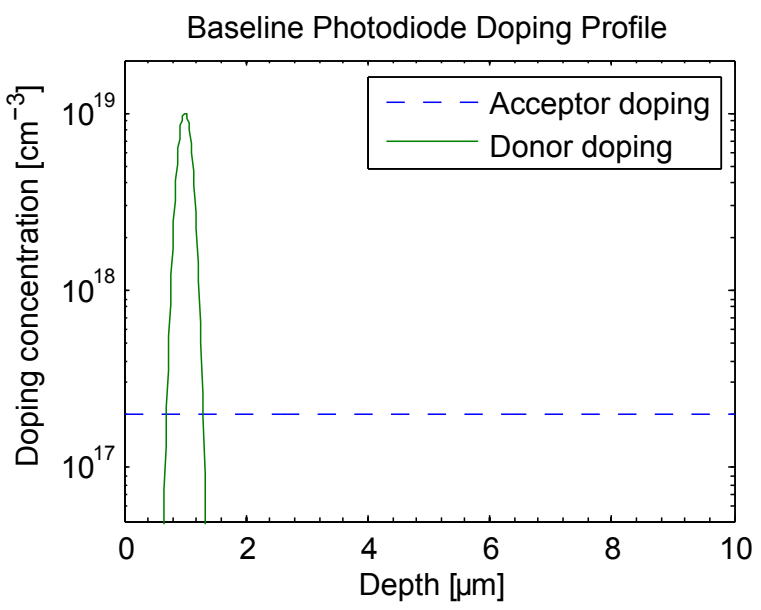

(b) Doping profile along center axis $(\mathrm{X}=0 \mu \mathrm{m})$

Figure 3.16: Baseline Sentaurus simulation structure and doping levels.

\section{Baseline Simulation Results}

Prior to implementing the 705S structure, a simple 2D substrate diode was simulated to obtain a baseline for comparison. The structure and simulation input files were parameterized to allow various junction depths, pixel sizes, biasing conditions, and range and direction of incident ionizing radiation to be simulated. Figure 3.16(a) presents the structure, depletion region (white), and ionization from a particle penetrating $5 \mu \mathrm{m}$, while Figure 3.16(b) presents the doping profile. A relatively high P-type substrate doping of $2 \times 10^{17} \mathrm{~cm}^{-3}$ was used which is similar to the well doping in the 705S sensor. The photodiode has a peak N-type doping of $1 \times 10^{19} \mathrm{~cm}^{-3}$. A $10 \mu \mathrm{m}$ depth was simulated to reduce the number of meshing nodes in the simulation.

Figure 3.17 presents results for a $1 \mu \mathrm{m}$ wide pixel with increasing range of the normally incident particle. The results show that the largest potential change is seen for a surface junction device due to the lower total capacitance inherent to the structure. Charge collection, however, improves for deeper junctions as charge 


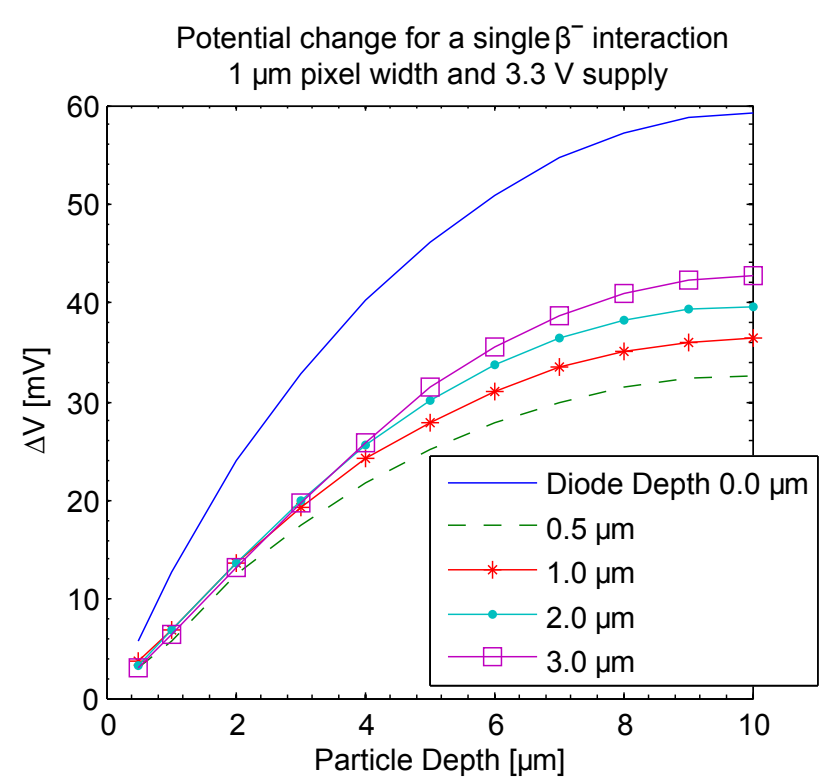

(a) Change in voltage

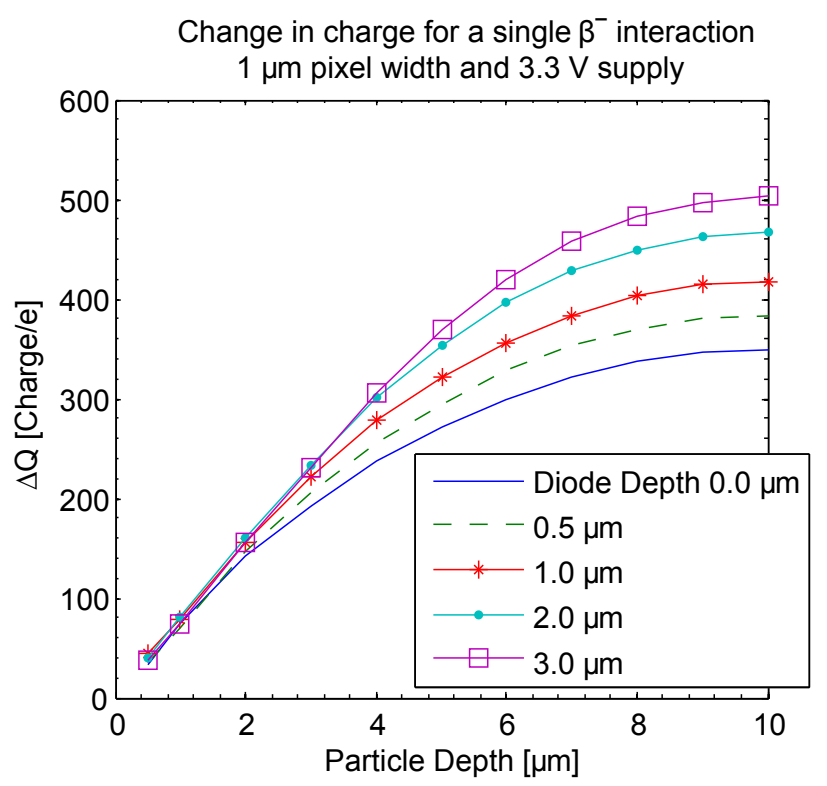

(b) Change in charge

Figure 3.17: Sentaurus simulation of a $1 \mu \mathrm{m}$ junction diode for increasing range of the incident particle.

deposited both above and below the junction has an opportunity to be collected prior to recombination. Charge collection would, however, reach a limit as ionized charge generated deep in the bulk would recombine before reaching the photodiode junction. For incident radiation with a range less than $3 \mu \mathrm{m}$, and in the absence of any simulated defects or interface states, the charge collection efficiency was $80 \%$ or better.

\section{S Simulation Results}

Early simulation results with the 705S structure showed that charge generated outside the P-well was not collected. For this reason, in the devices presented here, the simulation structure does not include a significant amount of bulk silicon. Figure 3.18 presents both the 2D and 3D 705S simulation structures. For the 2D structure, three $3 \mu \mathrm{m}$ wide N-type buried photocathodes, $1 \mu \mathrm{m}$ deep, sit inside a double implanted Pwell, with a pixel pitch of $6.4 \mu \mathrm{m}$. A $10 \mu \mathrm{m}$ long ionization track is shown. Gaussian 
doping profiles are used to approximate both the well and photodiode doping. For the 3 D structure, a $3 \times 3$ pixel structure was implemented with $3.2 \times 2.8 \mu$ m photodiodes. In both cases the central diode is the only one biased to $3.3 \mathrm{~V}$ through a $1 \mathrm{~T} \Omega$ resistor, while the rest are grounded. Figure 3.18(c) presents the doping profile as a function of depth through the central pixel. Both the substrate and P-well were grounded.

Simulations were carried out using the 2D models of the 705S sensor with normally, and transversally incident ionizing radiation and the results are presented in Figure 3.19. Figure 3.19(a) presents the results for normally incident ionizing radiation. It was seen that the amount of charge collected is halved when the radiation passes by at an offset of $3.2 \mu \mathrm{m}$ from the central pixel (at the boundary between two pixels). As charge would diffuse equally in all directions, this result is to be expected. When depositing $80 \mathrm{e}-\mathrm{h} / \mu \mathrm{m}$ nearly $100 \%$ of the charge was collected for path lengths less than $2 \mu \mathrm{m}$. Beyond $2 \mu \mathrm{m}$ depths, some of the charge is swept from the P-well into the substrate and is not collected, reducing the charge collection efficiency. For a $3 \mu \mathrm{m}$ path length, the worst case charge collection efficiency was simulated to be $70 \%$ when the incident radiation is centered on the pixel. No additional charge collection is seen when the path length of the radiation extends beyond the P-well. Figure 3.19(b) presents the results for transversally incident radiation at a depth of $1.5 \mu \mathrm{m}$. The results showed that the amount of charge collected increased linearly when simulating practical LET values, and that the charge collection neared a limit for $10 \mu \mathrm{m}$ path length. Taking the above observation into account, for charge generated within the active volume of the pixel, the worst case charge collection efficiency was found to be $70 \%$.

A coarser mesh was used when simulating the 3D model of the $705 \mathrm{~S}$ sensor to reduce the number of nodes and speed up simulation times. Charge collection within the active volume of the 3D model was nearly $100 \%$ for the same cases simulated with the 2D model. With a mesh size larger than the standard deviation of the Gaussian 


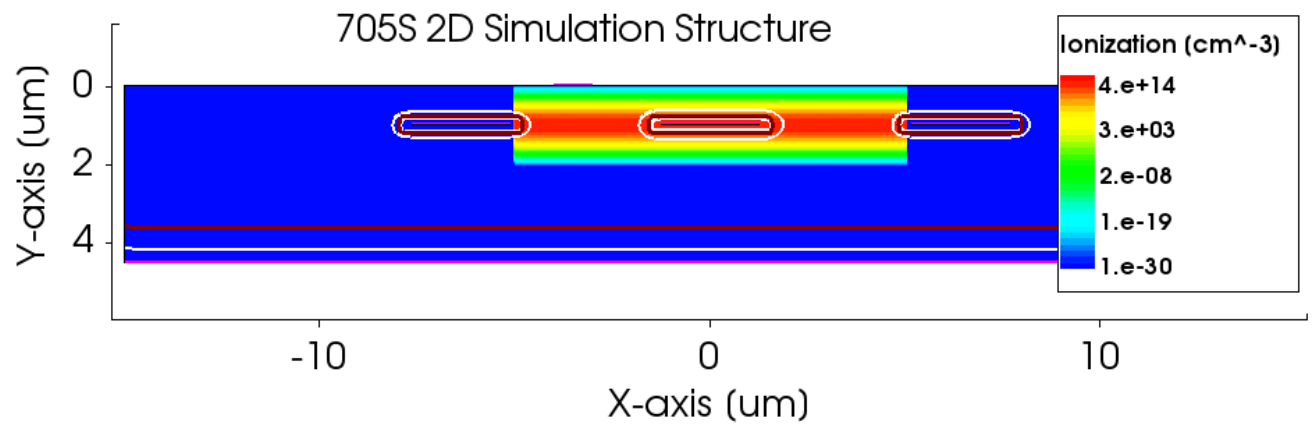

(a) Canon 705S 2D simulation structure showing depletion regions (bordered in white) and a transverse $10 \mu \mathrm{m}$ ionization track

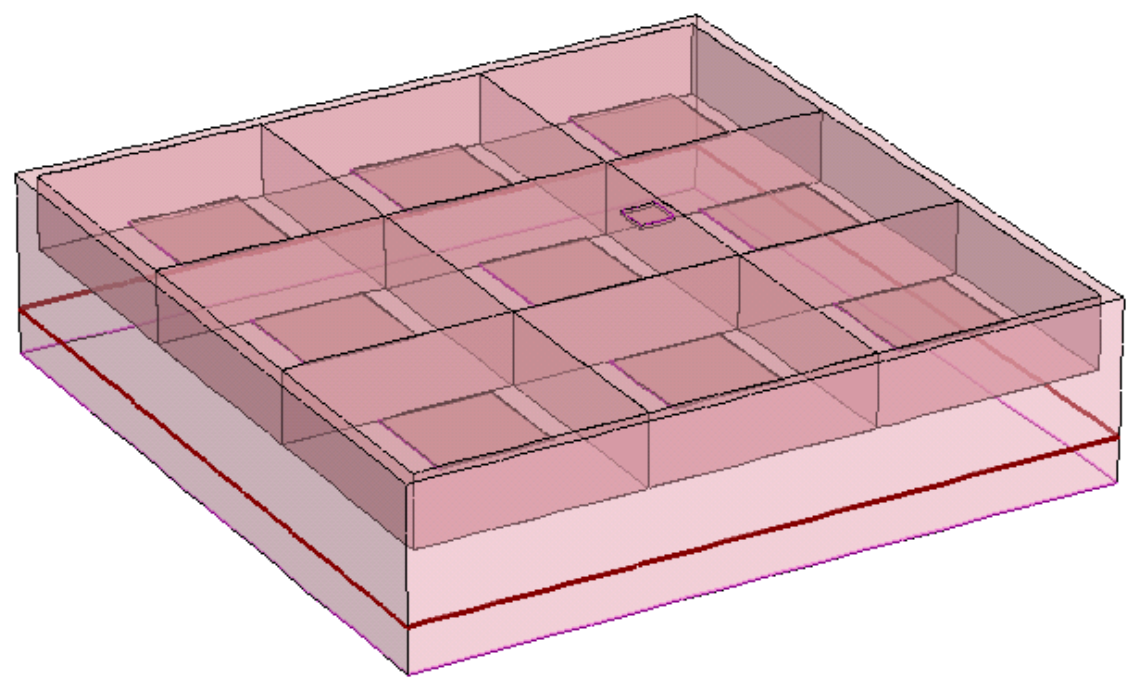

(b) Canon 705S 3D simulation structure

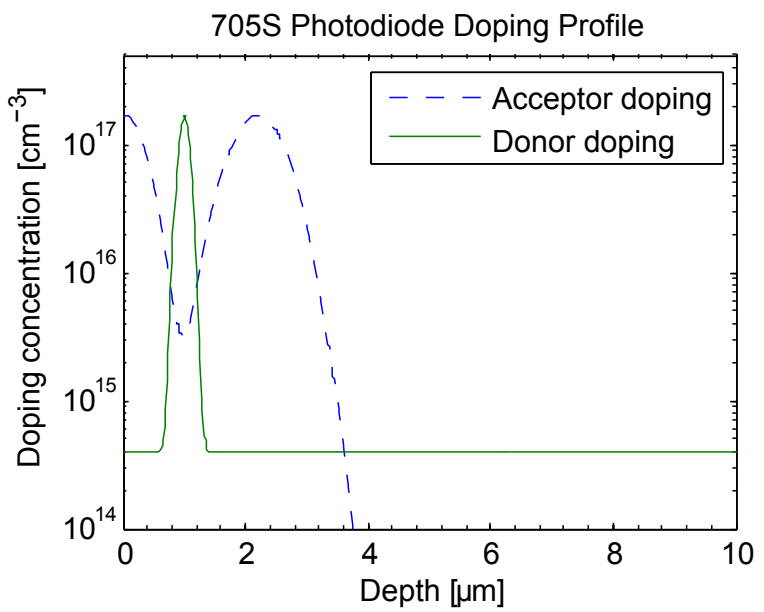

(c) Doping profile through central pixel

Figure 3.18: Sentaurus structures and doping profile used to model the Canon 705S CMOS MAPS. 


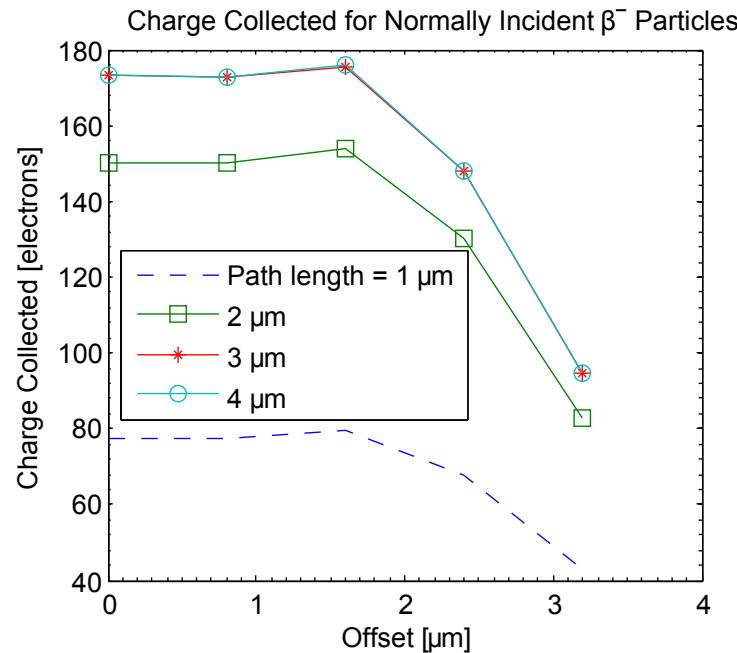

(a) Normally incident ionizing radiation offset from the central pixel

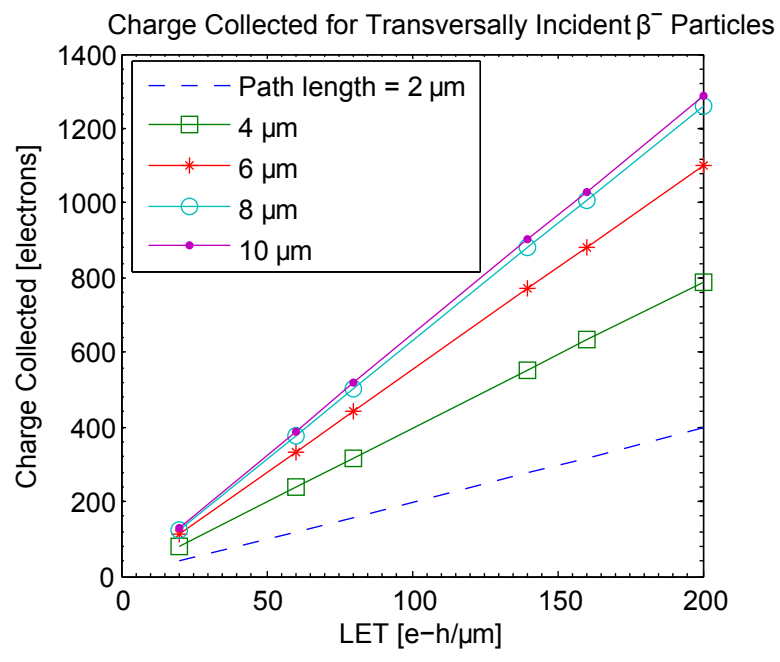

(b) Transversally incident ionizing radiation centered at the central pixel

Figure 3.19: $2 \mathrm{D}$ simulation results of the 705S MAPS showing the effects of strike offset and LET on charge collection for normally, and transversally, incident ionizing radiation.

distribution used for modelling the ionization, the 3D simulation lacked the ability to appropriately model the charge generated by the ionizing radiation. For this reason the worst case charge collection of $70 \%$ found in the $2 \mathrm{D}$ simulations was used when assessing the sensors usefulness in $\beta^{-}$detection.

In summary, simulations indicate that the Canon 705S CMOS MAPS, in the absence of crystal defects and interface states, could collect $70 \%$ or more of all charge created inside the $6.4 \times 6.4 \times 3 \mu \mathrm{m}$ pixel volume. A $\beta^{-}$particle following a linear trajectory would have a possible path length between 3 and $9 \mu \mathrm{m}$ inside the pixel volume. For the average stopping power of ${ }^{32} \mathrm{P}$ simulated in Section 3.2.3, including packaging effects, and a simulated charge collection efficiency of $70 \%$, the change in charge for an average $\beta^{-}$interaction would be 87 to 261 electrons. This is well above the read noise, and some particles will have a higher rate of energy deposition. In comparison to the baseline simulations, it is clear that a more sensitive detector could be made by increasing the charge collection volume, however, simulations showed that 
the 705S sensor would be able to detect the charge generated by some emissions from $\mathrm{a}^{32} \mathrm{P}$ radioisotopes including the effects of the sensor's packaging.

\subsection{Conclusion}

GEANT4 was used, in conjunction with its low-energy models, to simulate the passage of ionizing radiation in a silicon based sensor. Sentaurus, a semiconductor device simulator, was used to observe the electrical response of charge generation caused by the passage of ionizing radiation. Using these two tools it was predicted that a Canon 705S CMOS MAPS would be sufficient to detect the $\beta^{-}$emissions from ${ }^{32} \mathrm{P}$, and that it was not necessary to design a dedicated detector for the experiments involving $\beta^{-}$emitting isotopes in Chapter 4. Additionally, the effects of the 705S sensor's packaging were investigated. It was found that the system level spatial resolution would be quite coarse at $1.8 \mathrm{~mm}$, in part due to the package coverglass and air gap. It was found that in the presence of $590 \mu \mathrm{m}$ of glass, and a $750 \mu \mathrm{m}$ air gap, $15 \%$ of $\beta^{-}$emissions from a ${ }^{32} \mathrm{P}$ source would reach the sensor. It was also observed that $4 \%$ would backscatter out of the sensor, artificially inflating the number of $\beta^{-}$events observed. Lastly, Sentaurus revealed that the worst case charge collection efficiency for charge generated inside the active volume of the detector would be $70 \%$. Using the worst case charge collection efficiency, the amount of charge generated by an average ${ }^{32} \mathrm{P} \beta^{-}$emission in the active volume of the 705S MAPS, including packaging effects, would be well above the read noise of the detector. 


\section{Chapter 4}

\section{Quantitative detection of a $\beta^{-}$emitting isotope on a functionalized substrate using a CMOS sensor}

To address the desire for simple and direct quantitative analysis it is possible to use a solid state sensor to count the emissions from radiolabelled target molecules captured by probes. This method does not require an external excitation source nor an optical system for readout, is not subject to fluorescent resonance energy transfer (FRET), and is inherently quantitative.

Applications for this technique include biological and pharmaceutical studies, as well as characterization and measurement of specific affinity reactions and non-specific binding. This technology is particularly well suited for characterization of medicines developed for radioimmunotherapy (RIT).

For the proof of concept experiment reported herein, ${ }^{32} \mathrm{P}$ labelled adenosine triphosphate (ATP) was captured by an aptamer attached to a silanized glass substrate. Emissions from the ${ }^{32} \mathrm{P}$ were detected using a commercial CMOS monolithic active pixel sensor (MAPS) $[12,13]$.

Through the characterization of a commercial CMOS image sensor, the analysis of 
the image sensor packaging from Chapter 3, and a detailed experimental procedure, it will be shown that a direct quantitative measurement of radiolabelled biomolecules bound to a functionalized substrate can be made simply with minimal post processing.

\subsection{Experimental}

\subsubsection{Sensor Characterization}

Experiments were carried out using an 8 megapixel (MP) commercial CMOS MAPS from a Canon 350D digital SLR camera. Markings on the image sensor packing include the "705S" model name. This sensor is readily available, is cost effective and offers acceptable performance for this application. Additionally, all the necessary support electronics are already in place inside the 350D body. The sensor size is $22.2 \times 14.8 \mathrm{~mm}$ with a $6.4 \mu \mathrm{m}$ pixel pitch.

As designed, the 350D body is not optimized for the detection of ionizing radiation. There are a number of absorbing layers which, although not a problem in visible light photography, limit the detection efficiency for ionizing radiation. Of particular note are the IR filter and package coverglass.

An unmodified 350D was found to be weakly sensitive to $\beta^{-}$emissions from a ${ }^{90} \mathrm{Sr}$ source $\left(E_{a v}=196 \mathrm{keV}, E_{\max }=546 \mathrm{keV}\right)$, which has ${ }^{90} \mathrm{Y}\left(E_{a v}=937 \mathrm{keV}\right.$, $\left.E_{\max }=2280 \mathrm{keV}\right)$ as its progeny. The source was suspended inside the camera body just above the shutter, and 30 second exposures were taken at ISO1600. RAW data from the camera was converted from Canon's CR2 file format to portable gray map (PGM) using DCRAW and analyzed in MATLAB.

Fixed pattern noise (FPN) was measured by averaging 20 dark frames at the same exposure settings. This averaged dark frame was then subtracted from each frame with the source present. This has the additional effect of removing pixel bias (signal 
offset/digital offset bias/black offset level) which was measured to be 255 analog to digital units (ADU) on average.

As the MAPS uses 12 bit analog to digital converters (ADC), the maximum pixel value is 4095 ADU. Removing pixel bias then results in a signal dynamic range of 3840 ADU. A $\beta^{-}$particle passing through the substrate will generate charge which can be collected by one or more pixels. A pixel was considered struck when the amount of charge collected passed a set threshold. Initially this threshold was set at $20 \%$ of the maximum pixel value $(0.2 \times 4095 \mathrm{ADU}=819 \mathrm{ADU})$. Choosing a $20 \%$ threshold for detection was an arbitrary decision which allowed for a reasonable signal to noise (SNR) ratio. With FPN removed, no more than 10 pixels registered above threshold in the absence of the ${ }^{90} \mathrm{Sr}$ source. With the source in place approximately 55 pixels were raised to above threshold in a $30 \mathrm{~s}$ exposure. As was discussed in Section 3.2.3 it is the $\beta^{-}$from ${ }^{90} \mathrm{Sr}$ progeny, ${ }^{90} \mathrm{Y}$, which are being detected. Also note that for a sensor of this size operated at sea level, and assuming $100 \%$ quantum efficiency, less than 1 count for a $30 \mathrm{~s}$ exposure could be expected on average due to cosmic ray based radiation [101]. Without a source present it was possible to achieve 0 counts in a $30 \mathrm{~s}$ exposure by using a detection threshold of $80 \%$.

To improve the detection efficiency, the $350 \mathrm{D}$ was disassembled and the IR filter, a coated $2.51 \mathrm{~mm}$ thick piece of glass, was removed from atop the image sensor. Using the same experimental conditions, approximately 9650 pixels were raised to above a $20 \%$ threshold in the presence of the ${ }^{90} \mathrm{Sr}$ source for the same 30 s exposure. Thus, removing the IR filter results in 175 times more pixels registering above threshold.

With the IR filter removed and no source present, exposure times from 5 to $30 \mathrm{~s}$ were evaluated. For longer exposures, the mean of the ADC spectrum shifted to higher values. Using a 6.5\% threshold for 30 s exposures, no more than 150 pixels were activated, and only 25 had leaked to full ADC value. Due to the low dark count rate, and to minimize the amount of data collection required, exposure times of $30 \mathrm{~s}$ 
were used.

During these proof of concept experiments, the package coverglass $(0.49 \mathrm{~mm})$ was left covering the sensor. As a result it was desirable to utilize a relatively high energy $\beta^{-}$emitting isotope to maximize the number of detected emissions. Phosphorus-32 is a commonly used $\beta^{-}$emitting isotope with average and endpoint energies of 690 and $1710 \mathrm{keV}$, respectively, and a half life of 14.3 days. Adenosine triphosphate (ATP) labelled with ${ }^{32} \mathrm{P}$ was therefore used in these experiments. ATP labelled with ${ }^{32} \mathrm{P}$ is commercially available and an aptamer is available for its capture.

Simulations were performed using GEANT4 in Chapter 3 to calculate the number of $\beta^{-}$particles from the ${ }^{32} \mathrm{P}$ that would reach the sensor as well as pass through, and backscatter out of, the image sensor surface. These simulations were performed in Section 3.2.3 and it was found that that $15 \%$ of incident $\beta^{-}$particles would reach the sensor, but including double counting from backscattering out of the sensor, $19 \%$ of the total number of events pass through the interface layer. Hence, those simulation results indicated an artificial increase of $4 \%$ in the number of counts observed.

\subsubsection{Experimental Procedure}

Detection and analysis of selectively attached radiolabelled biomolecules was the intent of this experiment. Biomolecule attachment was achieved using an ATP aptamer (5'- $\mathrm{NH}_{2}-\left(\mathrm{CH}_{2}\right)_{6}$-ACC TGG GGG AGT ATT GCG GAG GAA GGT-3') synthesized at Carleton University. Note that aptamer binding is but one selective affinity reaction applicable to this experiment; many others are possible. Examples include single-stranded DNA hybridization and avidin-biotin binding interactions.

Round silica glass coverslips, $80-130 \mu \mathrm{m}$ thick and $5 \mathrm{~mm}$ radius, were cleaned then functionalized for aptamer attachment via reaction with 10-10undecenyltrichlorosilane. The resulting silane derived monolayer was oxidized to create terminal carboxylic acids which were activated for covalent attachment of the 

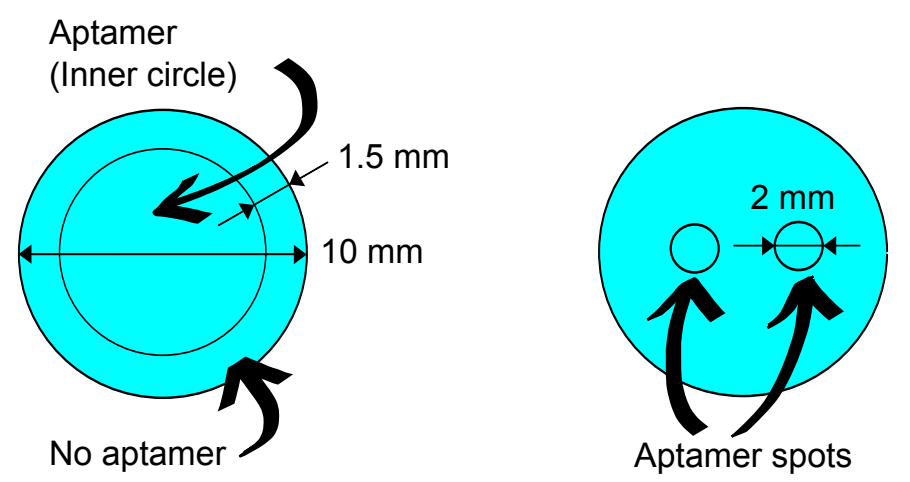

Figure 4.1: Round $5 \mathrm{~mm}$ radius coverslip, 80-130 $\mu \mathrm{m}$ thick, spotted with one or two aptamer regions via a silane based chemistry.

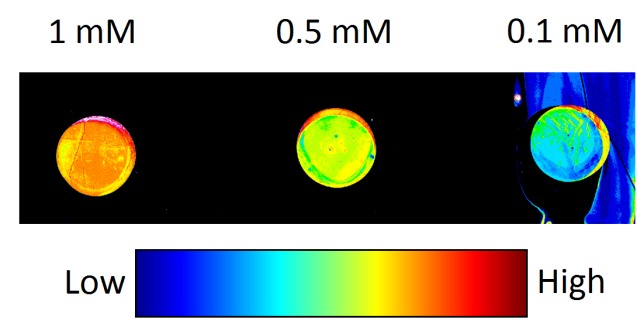

Figure 4.2: Fluorescence measurements of aptamer functionalized surfaces at $1 \mathrm{mM}$, $0.5 \mathrm{mM}$ and $0.1 \mathrm{mM}$ ATP concentrations.

amine modified aptamers [85]. Figure 4.1 depicts how the coverslips were prepared with one or two aptamer spots with radii of approximately 3.5 and $1 \mathrm{~mm}$, respectively. Due to 705S packaging, the system level spatial resolution of $1.8 \mathrm{~mm}$, simulated in Chapter 3, necessitated the use of large, well spaced, spots.

Fluorescence measurements were then used to qualitatively verify the success of the aptamer functionalization using Cy3 labelled ATP. Figure 4.2 presents fluorescence measurements at $1,0.5$ and $0.1 \mathrm{mM}$ ATP concentrations respectively. Note that at $0.1 \mathrm{mM}$ concentrations the fluorescent signal is weak.

Phosphorus-32 labelled ATP was purchased from American Radiolabeled Chemicals Inc. (Product \#: ARP0102D-1) and the emissions counted using the 705S CMOS MAPS from the 350D. Note that in this product only $50 \%$ of ATP molecules are labelled. For emission counting purposes, energy resolution was not necessary so the 


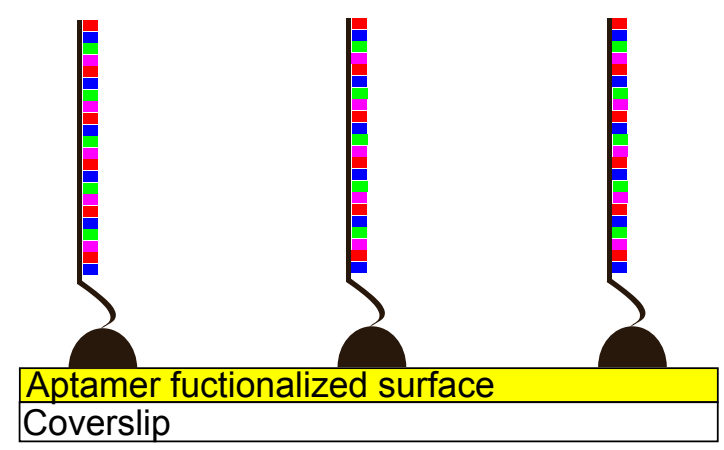

(a) ATP aptamer attached to coverslip

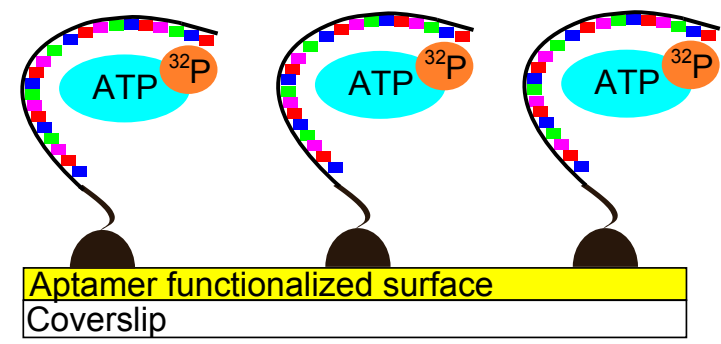

(b) Radiolabelled ATP captured by aptamer

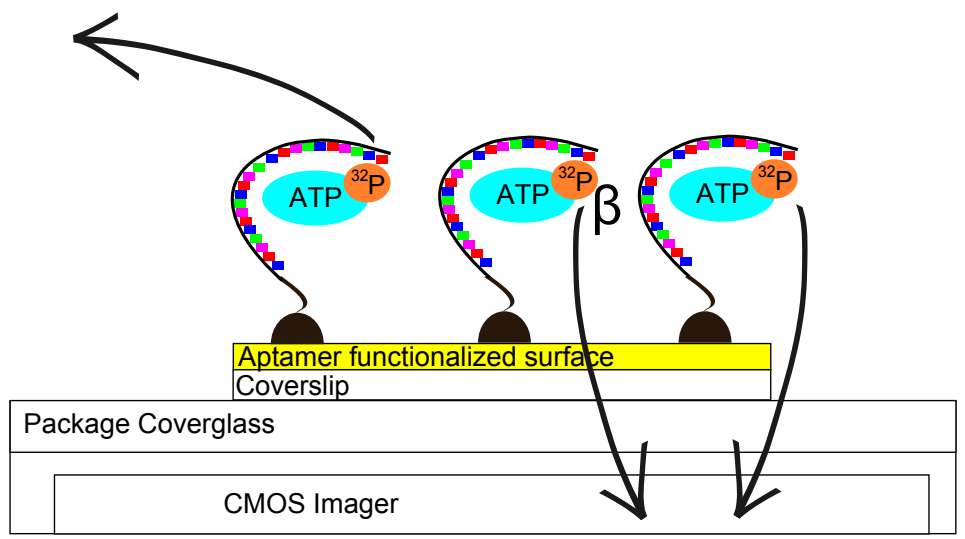

(c) $\beta^{-}$emissions detected with a CMOS image sensor

Figure 4.3: $\beta^{-}$labelled experiment procedure.

sensor was operated at its maximum gain setting (ISO 1600).

Before experimenting with the functionalized substrates, the quantum efficiency (QE) of the system was characterized. A known volume of ${ }^{32} \mathrm{P}$ labelled solution was pipetted onto a coverslip and the buffer solution left to evaporate. Once dry, the $\beta^{-}$ emissions were counted and the quantum efficiency of the system was estimated.

All data were recorded with the sensor completely shielded from light. Figure 4.3 provides a pictorial representation of the full experimental procedure while a more detailed description follows.

1. Prepare the slide by applying $80 \mu \mathrm{L}$ of binding buffer $(1 \mathrm{X} 3 \mathrm{M} \mathrm{NaCl}, 0.2 \mathrm{M}$ tris $\mathrm{pH} 7.6,0.05 \mathrm{M} \mathrm{MgCl}_{2}$ ) at 65 to $90^{\circ} \mathrm{C}$ to the top surface of the coverslip and allow to incubate for 10 minutes. 
2. Remove and discard binding buffer.

3. Add $80 \mu \mathrm{L}$ of $1 \mu \mathrm{M}{ }^{32} \mathrm{P}$ labelled ATP and allow to incubate 30 minutes. Note that only $50 \%$ of ATP molecules are labelled.

4. Recapture radiolabelled ATP solution.

5. Rinse with two treatments of $80 \mu \mathrm{L}$ of $1 \mathrm{X}$ phosphate buffered saline (PBS) and four treatments of $80 \mu \mathrm{L} 0.1 \mathrm{X}$ PBS, and recapture rinsing solutions.

6. Insert coverslip into camera body for measurement.

Raw data was captured using the following procedure. Note that all measurements were taken at room temperature without any additional sensor cooling. Also, although data was captured to observe read noise, the effect of read noise was not considered in data analysis. Rough measurements of read and thermal noise at ISO1600 resulted in values of less than 10 ADU, well below the threshold used for event counting.

1. Capture many dark frames to get the sensor and associated electronics up to operating temperature.

2. Capture 20 frames at the shortest exposure time. This is done to allow for the observation of read noise.

3. Capture 20 frames at the exposure time to be used with the sample absent. This allows FPN to be observed.

4. Capture 40 frames with sample present.

5. Capture 20 frames at the exposure time used with the sample absent. This allows for checking and averaging of FPN. 


\subsection{Experimental Results}

\subsubsection{Quantum Efficiency}

Measurements were taken with $2 \mu \mathrm{L}$ of $370 \mathrm{MBq} / \mathrm{mL}$ of ${ }^{32} \mathrm{P}$ solution dried on a glass coverslip. It was necessary to reduce the exposure time to $1 \mathrm{~s}$ in order to reduce the number of overlapping pixel clusters due to the relatively high activity of the sample. Averaged over 40 exposures, 6,934 counts/frame were measured at a 20\% threshold. Assuming the number of counts varies linearly with exposure time, this extrapolates to 208,029 counts/frame for a 30 s exposure. Given the activity on the coverslip was $740 \mathrm{kBq}$, the $\mathrm{QE}$ of the system was found to be $\eta_{\text {system }}=0.937 \%$ using a $20 \%$ counting threshold.

An experiment was carried out in order to validate the assumption that the image sensor's response scales linearly as a function of exposure time. A second identical $350 \mathrm{D}$ with the IR filter removed was exposed to a ${ }^{90} \mathrm{Sr}$ source at ISO1600 for exposure times of 1, 15, 25 and $30 \mathrm{~s}$, respectively. Data are presented in Figure 4.4. The average number of counts observed over 10 frames with FPN removed at a $20 \%$ detection threshold scaled linearly from 177, 2454, 3892 and 4886 counts, respectively, validating the assumption.

\subsubsection{Radiolabelled Measurements}

A limited number of samples remained after initial control and optimization experiments were carried out. Two samples of two spot ATP aptamer functionalized coverslips were available and underwent the testing protocol previously described. Measurement results are presented in Figure 4.5. Figures 4.5(a) and 4.5(b) show surface plots consisting of a summation of all 40 frames of data from samples 1 and 2 respectively. For ease of visualization the $2 \mathrm{D}$ surface plots are presented in $50 \times 50$ 


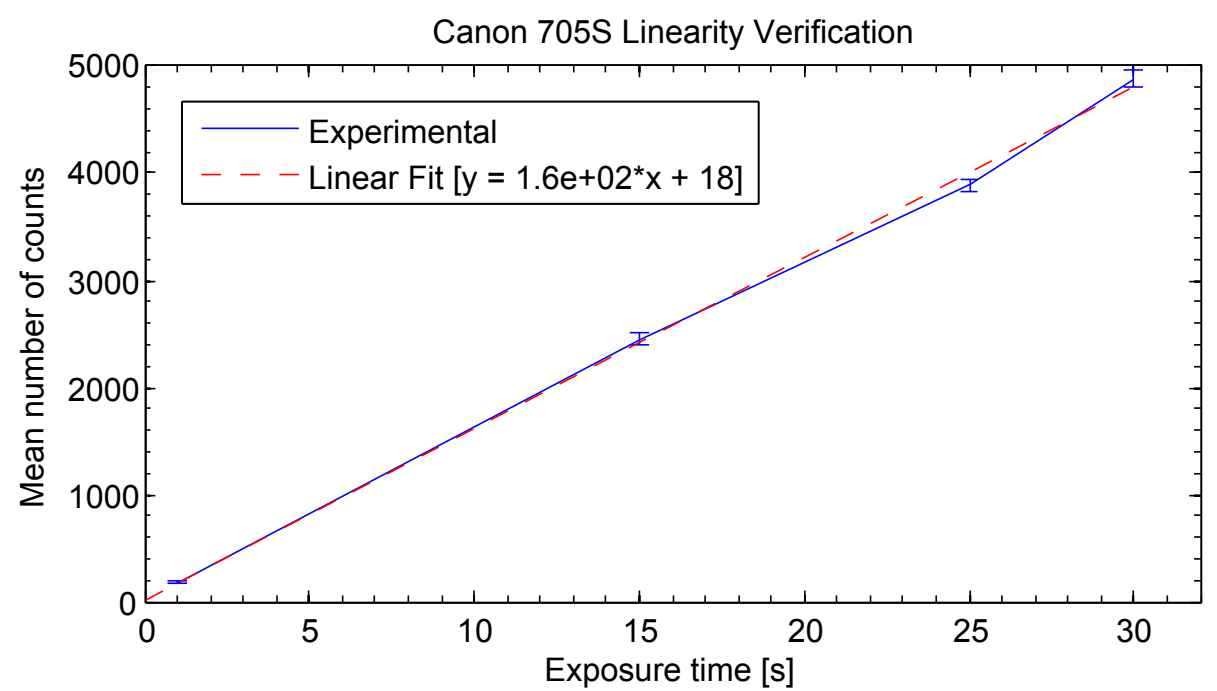

Figure 4.4: Experimental results showing the number of counts detected when exposing the 705S MAPS to an ${ }^{90} \mathrm{Sr}$ source for varying exposure times.

pixel bins and 1D plots through the peaks are provided in Figures 4.5(c) and 4.5(d) for samples 1 and 2, respectively. Both samples show two peaks of higher activity indicating specific binding of ATP on the spotted coverslips.

A $\beta^{-}$particle striking the MAPS can activate a cluster of pixels while interacting with the substrate. MATLAB was used for counting clusters of pixels above the set $20 \%$ threshold. Statistics regarding the data for samples 1 and 2 are provided in Figures 4.5(e) and 4.5(f) respectively. The statistics present the counts per image and pixel cluster size data. The counts per image in sample 1 are fewer compared to sample 2 due to the reduced activity of the second peak. Mean cluster size in sample 1 and sample 2 was calculated to be 1.13 and 1.12 pixels respectively.

\subsubsection{Background Calculation}

In order to ascertain the surface coverage it was necessary to subtract the background (non-specifically bound radiolabelled ATP) counts. Although background measurements were taken on bare and silanized glass, these measurements are not directly 


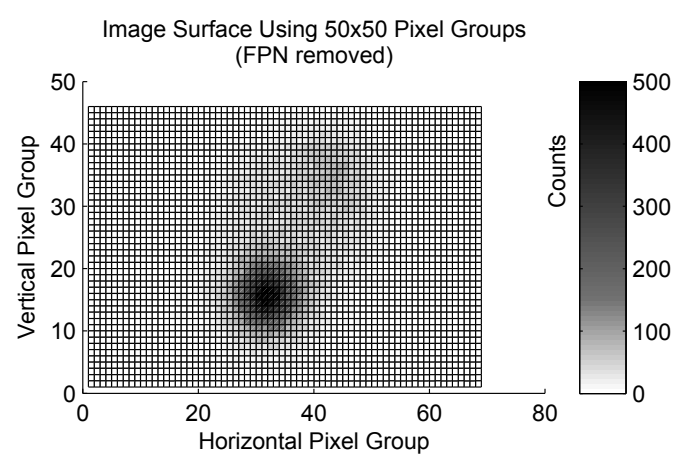

(a) Sample 1 surface plot

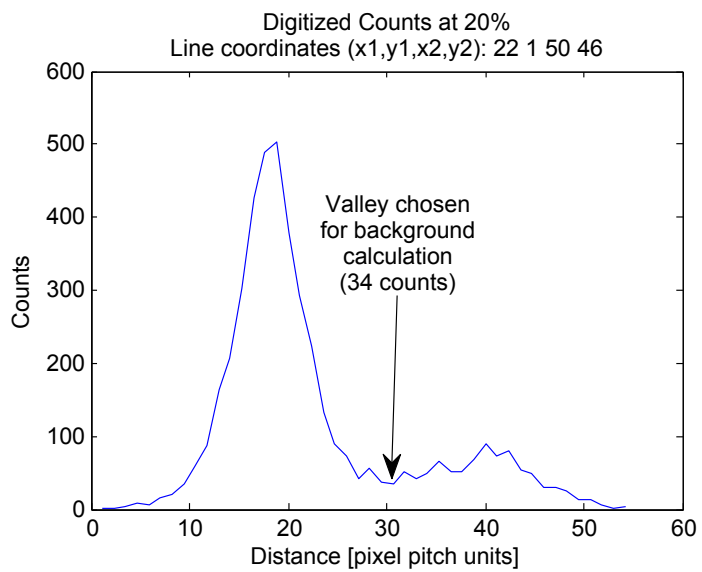

(c) Sample 1 line plot
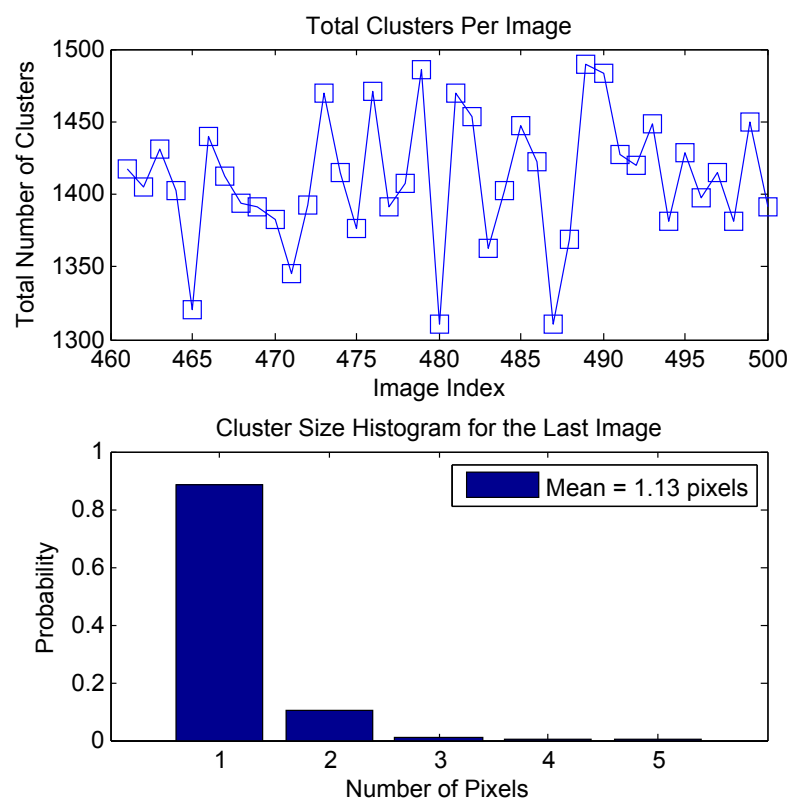

(e) Sample 1 histogram

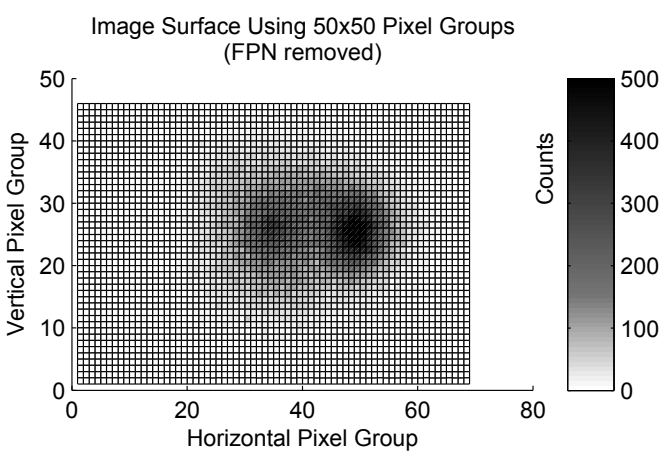

(b) Sample 2 surface plot

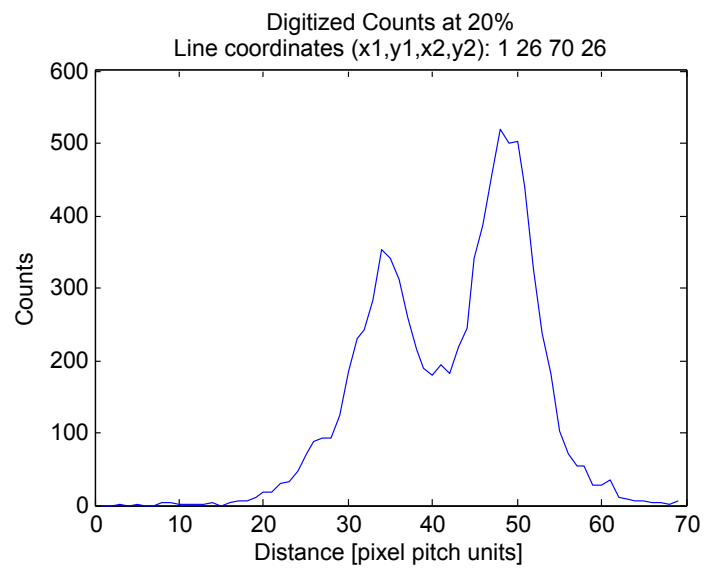

(d) Sample 2 line plot
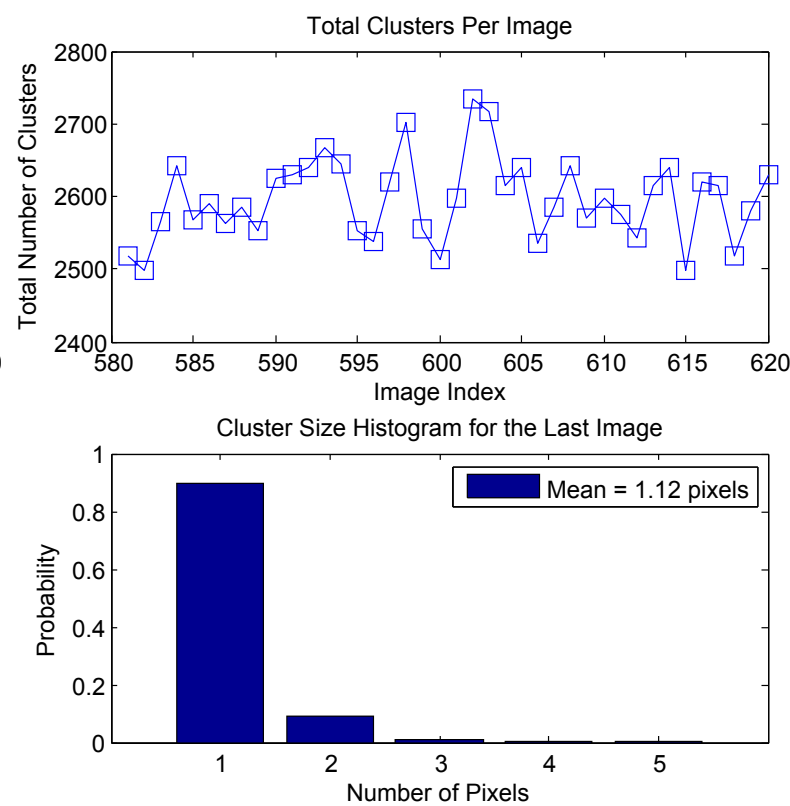

(f) Sample 2 histogram

Figure 4.5: Experimental results of ${ }^{32} \mathrm{P}$ labelled ATP selectively attached to an aptamer functionalized glass substrate and measured with a CMOS image sensor at room temperature. 
comparable to the measurements of the spotted samples as the potential for nonspecific ATP binding varies across bare, silanized, and DNA-modified glass surfaces.

In order to acquire a reasonable estimate of the background, a uniform activity of non-specifically bound ATP over the entire surface of the coverslip is assumed. The activity is calculated from the lowest point of the valley in Figure 4.5(c). A value of 34 counts/pixel group/40 frames was measured. Thus a $50 \times 50$ pixel group $\left(50 \times 50 \times(6.4 \mu m)^{2}\right)$ contributes 0.85 counts/pixel group/frame. The area of the coverslip $\left(\pi \times(5 \mathrm{~mm})^{2}\right)$ is equivalent to 767 pixel groups and thus a background count rate of 0.85 counts $/$ pixel groups $/$ frame $\times 767$ pixel groups $=652$ counts $/$ frame is assumed.

\subsubsection{Surface concentration of bound ATP}

Averaging the total counts per frame from the second spotted sample, there were on average 2596 counts per frame at a $20 \%$ threshold. The total number of counts from selective binding was then inferred to be $2596-652=1944$ counts/frame. From the quantum efficiency results, where 208,029 counts/frame were measured with a $740 \mathrm{kBq}$ source, the surface activity for 1944 counts/frame corresponds to $6.9 \mathrm{kBq}$. Assuming $1 \mathrm{~mm}$ radius spot sizes, the activity per area $\left(A_{\text {surface }}\right)$ is $110 \mathrm{kBq} / \mathrm{cm}^{2}$. Correcting for the system level quantum efficiency $\left(\eta_{\text {system }}=0.937 \%\right)$, the number of $\beta^{-}$particles which could be double counted $\left(\eta_{\text {Geant } 4}=4 \%\right)$ and only half of the ATP molecules being labelled $\left(\eta_{\text {labelling }}=50 \%\right)$, the surface concentration $\left(C_{\text {surface }}\right)$ was estimated to be $2.3 \times 10^{7}$ molecules $/ \mathrm{cm}^{2}$ using Equation 4.1 .

$$
C_{\text {surface }}=A_{\text {surface }} \times \frac{1}{\left(1+\eta_{\text {Geant } 4}\right)\left(\eta_{\text {system }}\right)\left(\eta_{\text {labelling }}\right)}
$$

A discussion of this result follows. 


\subsection{Discussion}

Both samples show two distinct regions of higher activity. However, sample 1 (Figure 4.5(a)) has a less pronounced second peak. Possible causes for the reduced activity in the second peak are non-uniform silanization, non-uniform aptamer coverage, and incomplete aptamer-ATP complexing. Fluorescence measurements, shown in Figure 4.2, confirm a non-uniform coverage. Aside from the non-uniform coverage, note that much higher concentrations of ATP were required in order to observe the fluorescence signal. Typically $5 \mathrm{mM}$ ATP solutions are used for characterizing fluorescence-based aptasensors $[102,103]$ and as can be seen in Figure 4.2 the $0.1 \mathrm{mM}$ solution was barely detectable. However, the radiolabelled method allowed detection of $1 \mu \mathrm{M}$ solution. In addition, the fluorescent sample only underwent two rinses at $1 \mathrm{X}$ PBS and one rinse at $0.1 \mathrm{X}$ PBS as compared to the four rinses employed in the radiolabel detection protocol.

Aptamer coverage was expected to be on the order of $\mathrm{pmol} / \mathrm{cm}^{2}\left(1 \times 10^{12} \mathrm{~cm}^{-2}\right)$ [104]. However, ATP-aptamer binding is an equilibrium process where the number of bound ATP is related to the concentration of ATP molecules present in solution. When using a $1 \mu \mathrm{M}$ solution of ATP the aptamer binding sites will not be saturated [105]. A coarse estimate of the expected concentration of bound ATP can be calculated using the dissociation constant $\left(K_{d}\right)$ for ATP-aptamer complexes where

$$
K_{d}=\frac{[\text { ATP }][\text { Aptamer }]}{[\text { Aptamer ATPcomplexes }]}
$$

Using a fluid volume of $80 \mu \mathrm{L}, 1 \mu \mathrm{M}$ ATP concentration, $6 \mu \mathrm{M}$ dissociation constant [105], and assuming an aptamer surface concentration of $1 \mathrm{pmol} / \mathrm{cm}^{2}$, one active binding site per aptamer due to low test concentrations, and spot sizes with $1 \mathrm{~mm}$ radii the expected bound quantity is $6.3 \times 10^{9}$ molecules $/ \mathrm{cm}^{2}$. Note that this estimate assumes the aptamers are free in a small amount of solution $(80 \mu \mathrm{L})$ and the binding 
behaviour for a functionalized surface may be different.

There was an insufficient number of samples available for rigorous experimentation, though the evidence does suggest that parallel detection of radiolabelled biomolecules using a CMOS image sensor is not only a useful technique, but is also a good candidate for the study of non-specific binding given its ability to measure low surface concentrations.

Some similar work has been performed. One group has mentioned the potential for parallel detection of DNA using the GaAs version of the MEDIPIX sensor, but such an experiment has not been published [10]. In [86] a CCD image sensor was used to detect radiolabelled DNA hybrids. The bulk of comparable research appears to focus on autoradiography $(\mathrm{AR})[10,11,47,49,51,87-89]$. However in AR spatial resolution is a primary concern. This has led researchers to use low-energy $\beta^{-}$emitting isotopes in conjunction with specialized back-thinned detectors. However, in the work presented herein, spatial resolution requirements are more relaxed. Note that the detection of arrayed radiolabelled biomolecules differs from AR in that a specific affinity reaction binds particular biomolecules at specific location on a substrate's surface. In AR the radiolabels are simply constrained within a tissue sample placed on or near the detector surface.

As seen in Figure 3.13 the presence of the air gap reduces the spatial resolution of the system. Removing the coverglass and placing the coverslip immediately atop the sensor would improve the QE and allow for the use of less energetic emitters. As in the case of AR, a reduced air gap and use of low-energy emissions would improve the spatial resolution of the system. To maximize both spatial resolution and sensitivity, the surface of the sensor could be functionalized directly.

An ideal CMOS $\beta^{-}$detector would be as sensitive as possible, while also being cost effective. It would seem likely, given the broad spectrum of $\beta^{-}$emission energies, that the ideal detector will vary by isotope and application. For low-energy emitters 
minimizing the amount of absorbing material between the radioisotope and detector will be important. Back-thinned detectors appear promising for these applications. In general, small pixel sizes will help improve the potential change seen from $\beta^{-}$ interactions, while thicker epitaxial layers will help with charge collection. However, if spatial resolution is important for a particular application, thin epitaxial layers can actually be beneficial in minimizing charge spreading to nearby pixels. It may be possible, in high volume applications, to develop one-time-use CMOS detectors where the surface is functionalized directly using low-cost CMOS image sensors. In [106] a low cost CMOS webcam was observed to detect $\beta^{-}$emissions from a ${ }^{90} \mathrm{Sr} /{ }^{90} \mathrm{Y}$ source. In all cases performance can be improved by running the detectors in a cooled state, but this may be expensive and impractical for some applications.

A brief investigation into the counting statistics presented in Figure 4.5 showed that the variance for each individual frame obeyed a $\sqrt{N}$ dependence as would be expected.

\subsection{QE Simulation}

An additional simulation was run after the experiment with radiolabelled ATP to further validate the software written with GEANT4. The simulation involved modelling the QE experiment above. Plotting the measured results of the QE measurements using $50 \times 50$ pixel bins as before, with FPN removed, yields the symmetric single peaked distribution shown in Figure 4.6. Simulating a uniformly active circular glass coverslip atop the 705S packaging with GEANT4 resulted in a narrower distribution than observed in the experimental results. It was found that simulating an annular source produced a distribution that best matched the experimental results.

In order to better observe the source distribution, the package coverglass was carefully removed and the glass coverslip placed directly atop the image sensor. This 


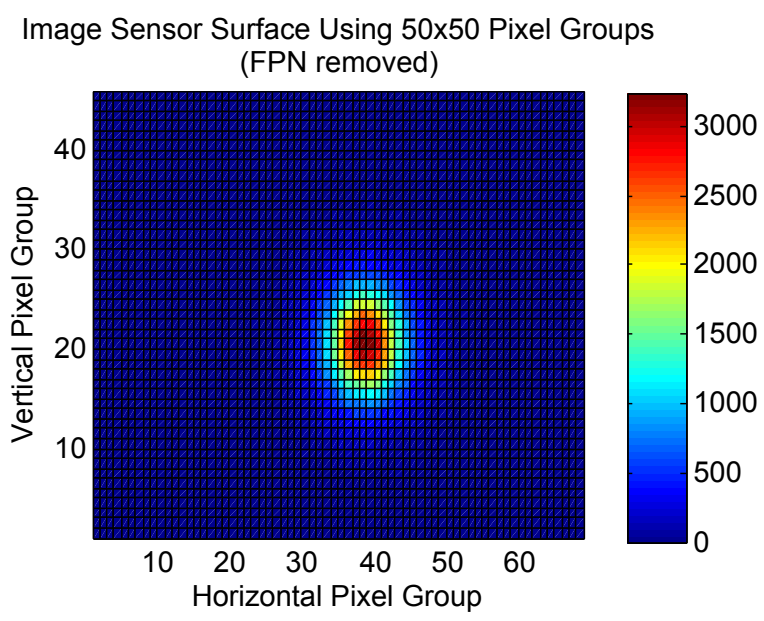

(a) Image sensor surface

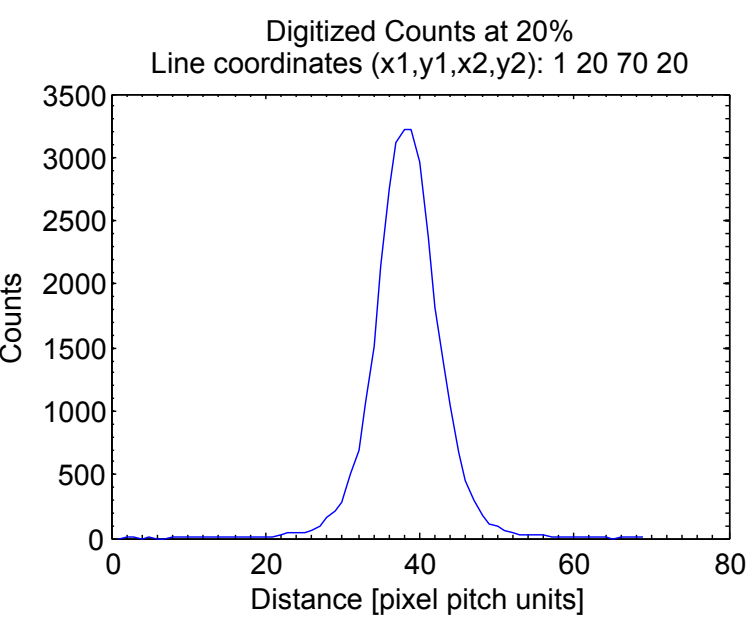

(b) Cross section

Figure 4.6: QE measurement results with a single ${ }^{32} \mathrm{P}$ spot on a glass coverslip.

reduced the loss in spatial resolution not only caused by scattering in the coverglass, but eliminated the air gap as well. Figure 4.7 presents raw data recorded from the 705S sensor with, Figure 4.7(a), and without, Figure 4.7(b), the coverglass in place. From Figure 4.7(b) the source pattern clearly demonstrates an annular pattern. This can be attributed to the "coffee ring" problem where the fluid dynamics of an evaporating drop can result in an increased concentration of solute depositing at the edges of the original droplet. This simulation demonstrated the potential ability of the software to retroactively determine the shape of the source distribution despite the effects of the detector's packaging.

\subsection{Conclusion}

For the first time a CMOS image sensor has been used to detect the presence of radiolabelled target biomolecules captured on a functionalized surface. Phosphorus-32 labelled ATP was successfully bound to aptamer functionalized glass coverslips and 


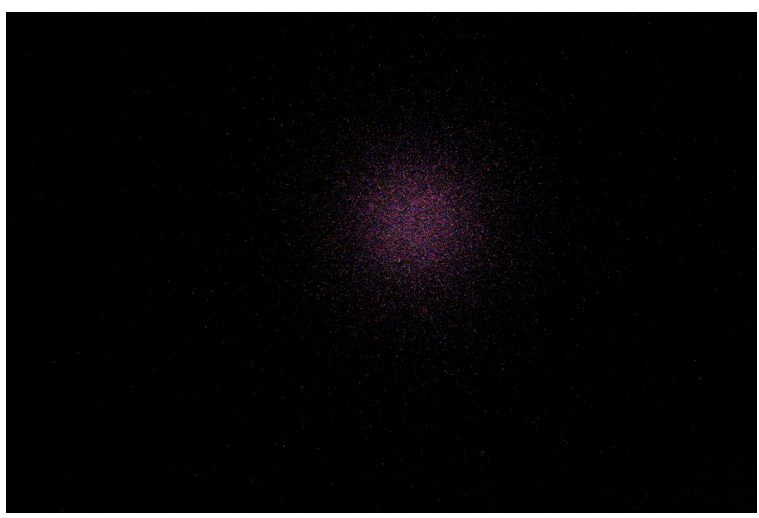

(a) Coverglass in place.

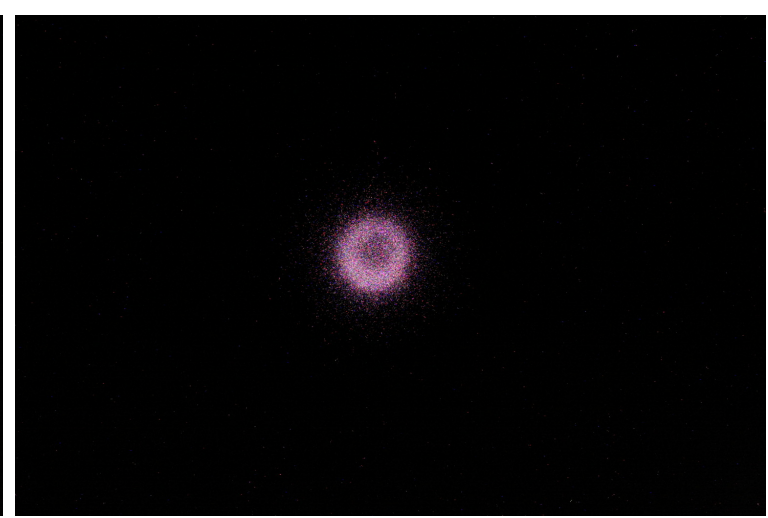

(b) Coverglass removed.

Figure 4.7: Raw QE measurement data with a single spot of dried ${ }^{32} \mathrm{P}$ labelled ATP on a glass coverslip.

the emissions detected with a CMOS MAPS at room temperature. The proof of concept results are promising and demonstrate the ability of this technique to directly and quantitatively measure low surface concentrations. A surface concentration of just $2.3 \times 10^{7}$ molecules $/ \mathrm{cm}^{2}$ was measured. These results were achieved with relatively little post processing and an inexpensive image sensor. Further study and exploration of the technique is merited. Also shown is the amenability of the technique to parallel detection of radiolabelled biomolecules.

This technique has applications in pharmaceutical research including RIT, nonspecific binding studies, and quantitative detection of biomolecules such as DNA and proteins.

Also demonstrated is the ability of the GEANT4 software to provide insight into the shape of the source distribution which would have been otherwise obscured by the detector's packaging. 


\section{Chapter 5}

\section{MOS testbed for the characterization of targeted $\alpha$ therapy pharmaceuticals}

Radioimmunotherapy (RIT), sometimes called targeted radionuclide therapy (TRT), has been successful in treating some cancers [71]. TRT utilizes molecules which exhibit a specific affinity interaction to deliver a radioactive payload to a targeted cell [83]. Various targeting agents have been explored including monoclonal antibodies (mAbs), antibody fragments, engineered proteins, protein scaffolds, peptides, tetramers and more recently aptamers $[71,72,83]$. Relative to antibodies, aptamers have a number of advantages including higher specificity, selectivity, and tumor penetration ability [71], as well as greater stability and ease of chemical modification. Pre-targeting methods have also been attempted where a cancerous cell is first labelled with an identifying molecule, and it is this identifying molecule that is targeted [72].

Both $\alpha$ and $\beta$ particle emitting isotopes are under investigation for TRT. In the case of $\alpha$ based TRT, also termed targeted alpha therapy (TAT), some isotopes of interest include bismuth-213 $\left({ }^{213} \mathrm{Bi}\right)$, astatine-211 $\left({ }^{211} \mathrm{At}\right)$, radium-223 $\left({ }^{223} \mathrm{Ra}\right)$ and actinium-225 $\left({ }^{225} \mathrm{Ac}\right)[30,72,75-77,83]$. Of these, ${ }^{225} \mathrm{Ac}$ is of particular interest as it generates four $\alpha$ particles before decaying to stable ${ }^{209} \mathrm{Bi}$, and because of this ${ }^{225} \mathrm{Ac}$ has been referred to as a nanogenerator [77]. 
When using $\alpha$ emitting isotopes various chelators can be used to attach the radionuclide to the targeting agent. EDTA, PEPA, DTPA, HEHA and DOTA are all chelators which have been used however 1,4,7,10-tetraazacyclododecane-1,4,7,10tetraacetic acid (DOTA) was found to be best for in-vivo applications $[72,74,76]$.

As $\alpha$ particles have a high linear energy transfer (LET), their range in biological matter is small. In general an $\alpha$ particle will deposit all of its energy within a few cells lengths. This results in a much higher localized dose, less damage to surrounding healthy tissue, and ability to use a significantly smaller dose than would be necessary to achieve the same effectiveness with a $\beta$ emitting isotope [30].

In Chapter 4 a proof-of-concept was carried out using a CMOS image sensor functionalized with aptamers to capture radiolabelled biomolecules from solution [13]. This method allowed for quantitative analysis of the radioactivity captured by the aptamers and could be used in the characterization of TRT pharmaceuticals using $\beta$ emitting isotopes.

In this chapter, a system is presented which can be used in the characterization of $\alpha$ emitting radioimmunotherapy pharmaceuticals. A metal-oxide-semiconductor (MOS) sensor was designed, fabricated, and tested by chelating an $\alpha$ emitting isotope in solution. A commercial sensor could have been used for this experiment, however, fabricating the sensor in-house allowed for full process control and the ability to perform wafer-level thin film deposition. The sensor consists of a $16 \times 16$ array of $100 \mu \mathrm{m}$ square $\alpha$ particle sensitive cells and has been fabricated in-house using a simple MOS process. A subset of those cells were functionalized with DOTA-DNA conjugates as the chelating agent to capture the radioisotope in solution as well as mimic an aptamer-based TAT agent. This type of system, and variations of it, could have applications in assessing chelation efficiency and targeting efficiency under a variety of biological conditions before advancing to animal trials. 


\section{$5.1 \quad$ Experimental}

This proof-of-concept relies on developing an $\alpha$ particle sensitive integrated circuit (IC) able to withstand exposure to the solutions and temperatures required for functionalization and target capture, and evaluating the chemistry to attach various $\alpha$ particle emitting radioisotopes. The subsections which follow present details on the IC processing, chemical attachment, and ultimately the experimental protocol used in testing.

\subsubsection{Sensor Design and Preparation}

Using a simple MOS fabrication process, an $\alpha$ particle sensitive IC was developed that borrows heavily from early dynamic random access memory (DRAM) design, and for this reason the IC has been dubbed " $\alpha$ RAM". A $16 \times 16$ array of $100 \mu \mathrm{m}$ square $\alpha$ particle sensitive cells is integrated with all necessary support electronics on a $3 \times 3 \mathrm{~mm}^{2}$ silicon substrate. Columns of the $\alpha$ RAM are polled sequentially and report whether or not a cell on that column was struck by an $\alpha$ particle. Previous characterization of the IC demonstrated a quantum efficiency of unity for normally incident $\alpha$ particle detection. Detailed information about the IC's design, operation and characterization have been presented elsewhere $[16,17]$. This particular version of the IC differs from that presented previously only in having independent outputs for each row of $\alpha$ particle sensitive cells.

In order to prepare the $\alpha \mathrm{RAM}$ for the following experiment, several additional processing steps were necessary. A low temperature oxide (LOTOX) film was deposited over the surface of the chip. This film acts as scratch protection, chemical barrier, and an insulating dielectric for the subsequently deposited film. Once the LOTOX was deposited, contact windows to the bond pads were patterned and opened. 


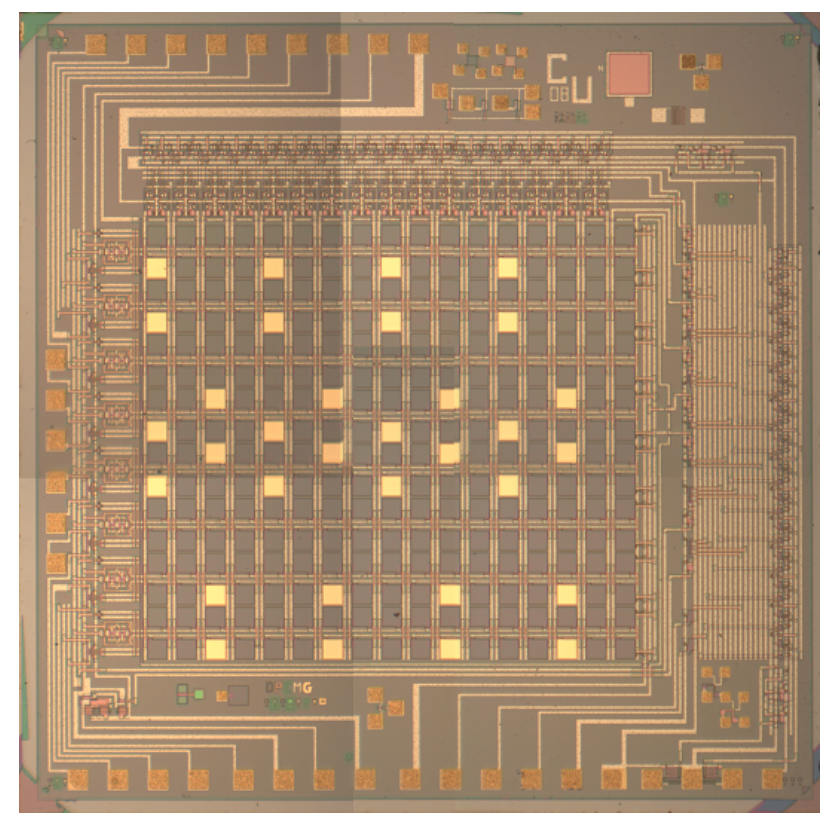

(a) Processed $\alpha \mathrm{RAM}$

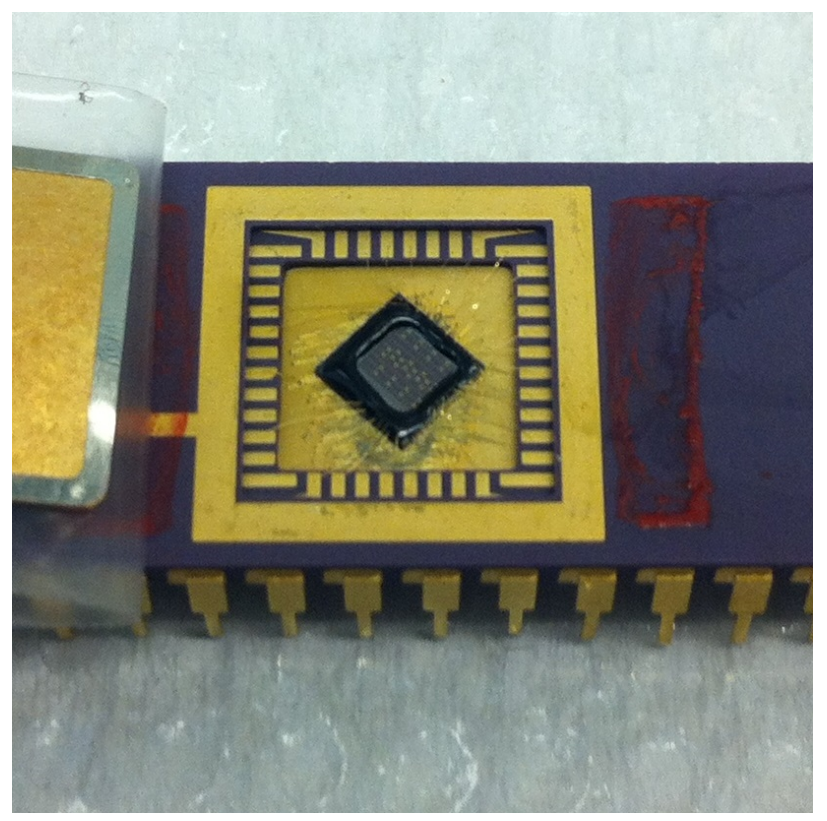

(b) Packaged $\alpha$ RAM with bond pad protection

Figure 5.1: Images of a fully processed and packaged $\alpha$ RAM. Cells are indexed from top left $(1,1)$ to bottom right $(16,16)$. Wirebonds on rows 7 and 16 were broken accidentally during handling. Aside from the missing rows, all cells are functional.

Once opened, a thin chrome/gold stack was deposited and patterned by a lift-off process covering all bond pads and a select number of cells on the IC. The thickness of the LOTOX, chrome, and gold were 370, 5 and $390 \mathrm{~nm}$ respectively. Figure 5.1(a) presents the fully processed $\alpha$ RAM. Note that the surface functionalization discussed in Section 5.1.2 will attach chelating agents on the gold squares present on the $\alpha$ RAM surface. This method of functionalizing only gold coated cells will be referred to as self-spotting.

Once processing was complete, the chips were packaged and wirebonded. Due to pinholes in the chrome/gold stack, it was found to be necessary to protect the bond pads around the periphery of the chip from the functionalization chemistry. This was achieved by melting APIEZON Wax $\mathrm{W}^{\circledR}$ and covering the bond pads as seen in Figure 5.1(b). 
A custom microcontroller based readout board was developed for the $\alpha$ RAM which gathers data and then outputs them to a serial terminal. The data from the terminal were logged in a text file for later analysis. MATLAB was used to parse and analyze the data.

An updated version of the IC with similar capabilities is currently being developed using the commercial CMOS foundry Austria Microsystems (AMS). This development is discussed in more detail in Section 5.2.4 below.

\subsubsection{Chemistry}

A subset of cells on the $\alpha$ RAM surface were overlaid with a gold film, allowing a gold-thiol chemistry to be used to functionalize these cells with DOTA using doublestranded DNA as a tether. One strand of the DNA is prepared with a sulfhydryl group for attachment to the gold, while the complementary DNA strand is functionalized with the chelator.

\section{Preparation of the DOTA Chelator DNA Conjugate (DC-DOTA)}

The DNA tether sequence (DT) (5'-CCA CTA CCG GGG CAC GTC CA-3') and its complement, DNA chelator sequence (DC) (5'-TGG ACG TGC CCC GGT AGT GG-3') were purchased from Integrated DNA Technologies (Coralville, USA), both 5'-disulfide modified and HPLC purified. To cleave the 5'-disulfide and prepare DC for conjugation with the chelator, maleimido-mono-amide-DOTA, (Macrocyclics: Dallas, USA), DC (150 nmol) was dissolved in $75 \mu \mathrm{L}$ of $50 \mathrm{mM}$ Tris (pH 8.4) containing $100 \mathrm{mM}$ dithiothreitol (DTT) and vortexed at ambient temperature for $30 \mathrm{~min}$. DC was purified and buffer exchanged into 50 mM HEPES (pH 7.0) using Micro Bio-Spin Chromatography columns (Bio-Rad, Canada) as per the manufacturer's instructions and then concentrated to $100 \mu \mathrm{L}$ using Amicon Ultra $0.5 \mathrm{~mL}$ Centrifugal Filters. DC was then reacted with a large excess $(1.27 \mu \mathrm{mol})$ of maleimido-mono-amide-DOTA in 
$300 \mu \mathrm{L}$ of $50 \mathrm{mM}$ HEPES ( $\mathrm{pH}$ 7.0) for 3 hours, at ambient temperature. Following chelation, DC was desalted to remove unreacted maleimido-mono-amide-DOTA. DCDOTA was quantified by UV-Vis $\left(\lambda_{\max }=260 \mathrm{~nm}\right)$ and the mass of the conjugate was confirmed by ESI Mass spectrometry (Novatia).

\section{Preparation of the DNA Tether (DT-SH)}

Cleavage of the $5^{\prime}$-disulfide was performed by dissolving DT $(150 \mathrm{nmol})$ in $75 \mu \mathrm{L}$ of $50 \mathrm{mM}$ Tris (pH 8.4) containing $100 \mathrm{mM}$ DTT. Following a $30 \mathrm{~min}$ ambient temperature incubation with vortexing, DT-SH was purified, buffer exchanged and concentrated as described for DC.

\section{Hybridization of the DNA Tether and DOTA Chelated Tether Comple- ment}

An equimolar $(1.75 \mathrm{mM})$ solution of the two sequences, DT-SH and DC-DOTA, was prepared by combining $60 \mu \mathrm{L}$ each. The sample was heated to $60^{\circ} \mathrm{C}$ for 5 minutes and then allowed to cool slowly to ambient temperature to promote hybridization of the complementary DNA sequences and the consequent formation of the stable, bi-functionalized duplex for deposition onto the gold surface.

\section{Surface Functionalization}

SigmaCote ${ }^{\circledR}$ (Aldrich) was used as a silanizing agent in an attempt to minimize nonspecific interactions of the DNA with the oxide surface. $10 \mu \mathrm{L}$ of the neat solution was applied to the IC and left at ambient temperature for 10 minutes, followed by a 20 minute bake at $100^{\circ} \mathrm{C}$. After extensive washes with deionized (DI) water (Milli Q water system, $18.2 \mathrm{M} \Omega$ ), $10 \mu \mathrm{L}$ of the DC-DOTA/DT-SH duplex DNA solution was deposited onto the surface and left at ambient temperatures for 12 hours. The surface was then subjected to DI water rinses $(10 \times 10 \mu \mathrm{L})$ and left to air dry. 


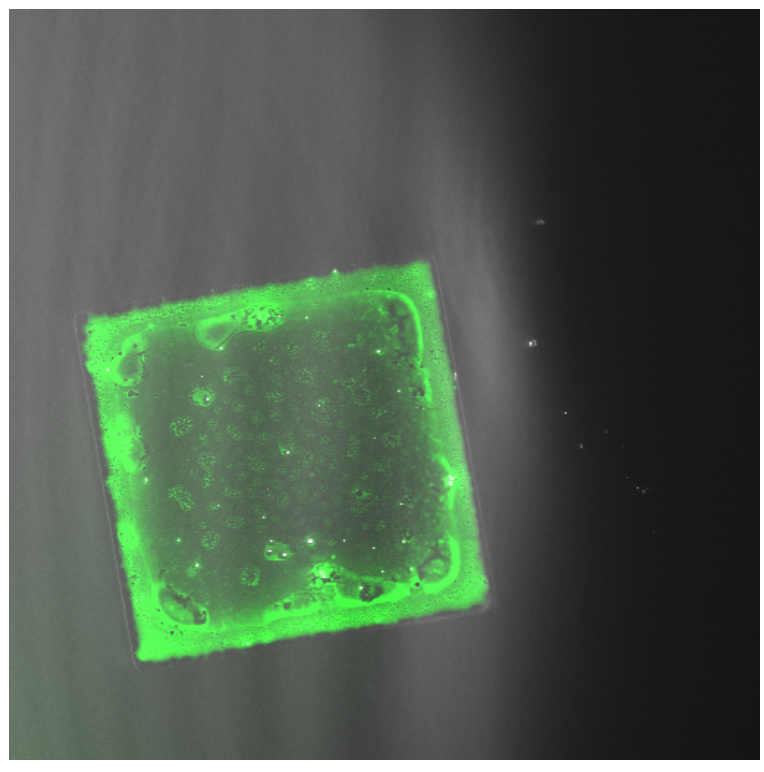

(a) Single self-spotted gold square

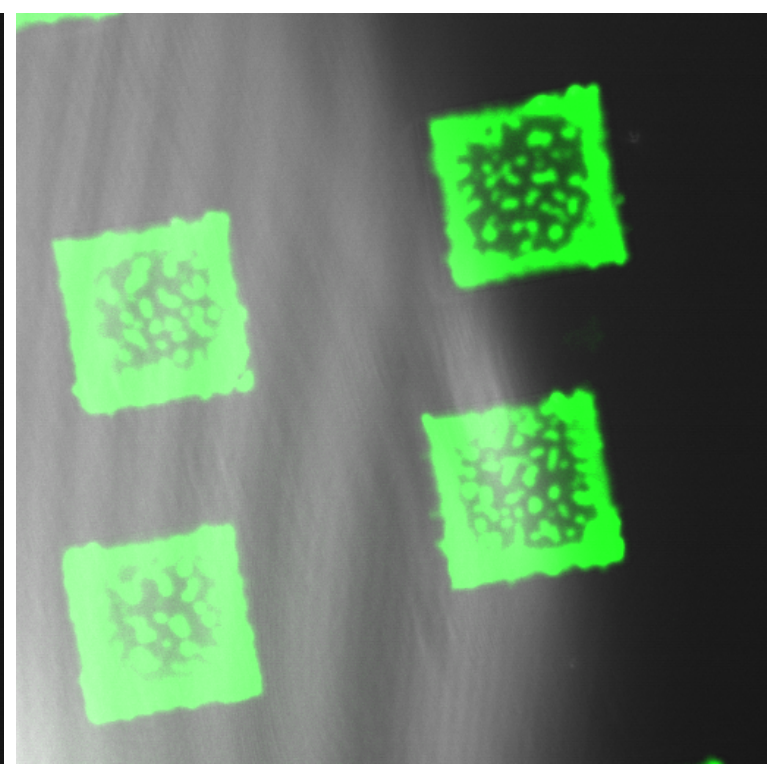

(b) Multiple self-spotted gold squares

Figure 5.2: Self-spotting verification with thiol modified single-stranded DNA and its Cy3 labelled complement on $100 \mu \mathrm{m}$ square gold patterned cells.

An early test was carried out to verify the self-spotting method. Thiol modified single-stranded DNA were introduced over an oxidized silicon wafer with a patterned $\mathrm{Ti} / \mathrm{Au}(20 / 25 \mathrm{~nm})$ array of $100 \mu \mathrm{m}$ square cells. Cy3 labelled complementary strand was then introduced and left to hybridize at room temperature. These wafers had been treated with SigmaCote ${ }^{\circledR}$, but were not heat treated prior to incubation. Washing the sample post hybridization drastically reduced the fluorescence signal. Figure 5.2 presents the fluorescence data supporting the self-spotting method. It was also observed that after functionalization and hybridization the Au film was less adhered to the $\mathrm{Ti}$ adhesion layer. It is believed that the $\mathrm{Ti}$ was able to react with the functionalizing and hybridization chemistry through pinholes in the Au film. To help reduce this problem, a thicker Au film was deposited on the $\alpha$ RAM ICs. The thicker gold film was not characterized in the same way due to the facilities not being available. 


\section{Americium Chelation}

In order to allow a larger time window for experiments, and also due to simplified licensing considerations, americium-241 $\left({ }^{241} \mathrm{Am}\right)$ was used as the radioisotope as opposed to typical isotopes of interest for TAT. While both being actinides, with a half life of 432 years, ${ }^{241} \mathrm{Am}$ is not well suited to TAT, while ${ }^{225} \mathrm{Ac}$ for example, with a much shorter half life of 10 days is of much greater interest [30].

A $100 \mu \mathrm{L}$ aliquot of americium chloride solution (Eckert \& Ziegler, $37 \mathrm{kBq} / 5 \mathrm{~mL}$, $0.1 \mathrm{M} \mathrm{HCl}$ ) was diluted with $400 \mu \mathrm{L}$ of $0.5 \mathrm{M}$ ammonium acetate buffer to bring the solution to $\mathrm{pH}$ 6. The americium solution $(10 \mu \mathrm{L})$ was then deposited onto the IC and left at ambient temperature for 2 hours, followed by DI water rinses $(5 \times 10 \mu \mathrm{L})$ and air drying.

\subsubsection{Experimental Protocol}

As a demonstration of the testbed for TAT assessment, ${ }^{241} \mathrm{Am}$ was chelated from solution where DOTA was selectively tethered to portions of the $\alpha$ RAM surface by means of thiolated DNA. All data were recorded with the sensor completely shielded from light. A pictorial representation of the experiment can be seen in Figure 5.3 while a written procedure follows.

1. Process and package the $\alpha$ RAM including wax bond pad protection.

2. Test $\alpha$ RAM operation for a minimum of 24 hours to verify any background counts and IC operation.

3. Apply SigmaCote ${ }^{\circledR}$ for 10 minutes prior to chelator attachment and cure with a 20 minute bake at $100^{\circ} \mathrm{C}$.

4. Re-test the IC for a minimum of 24 hours. 


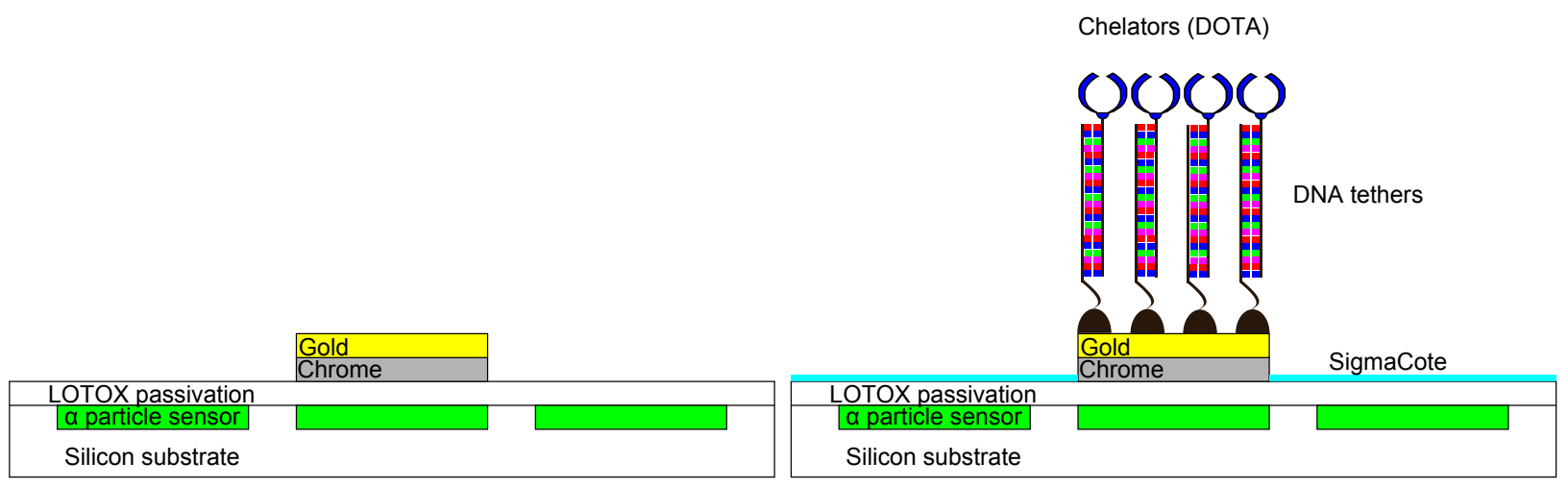

(a) Processed $\alpha$ RAM

(b) Surface functionalization and chelator attachment
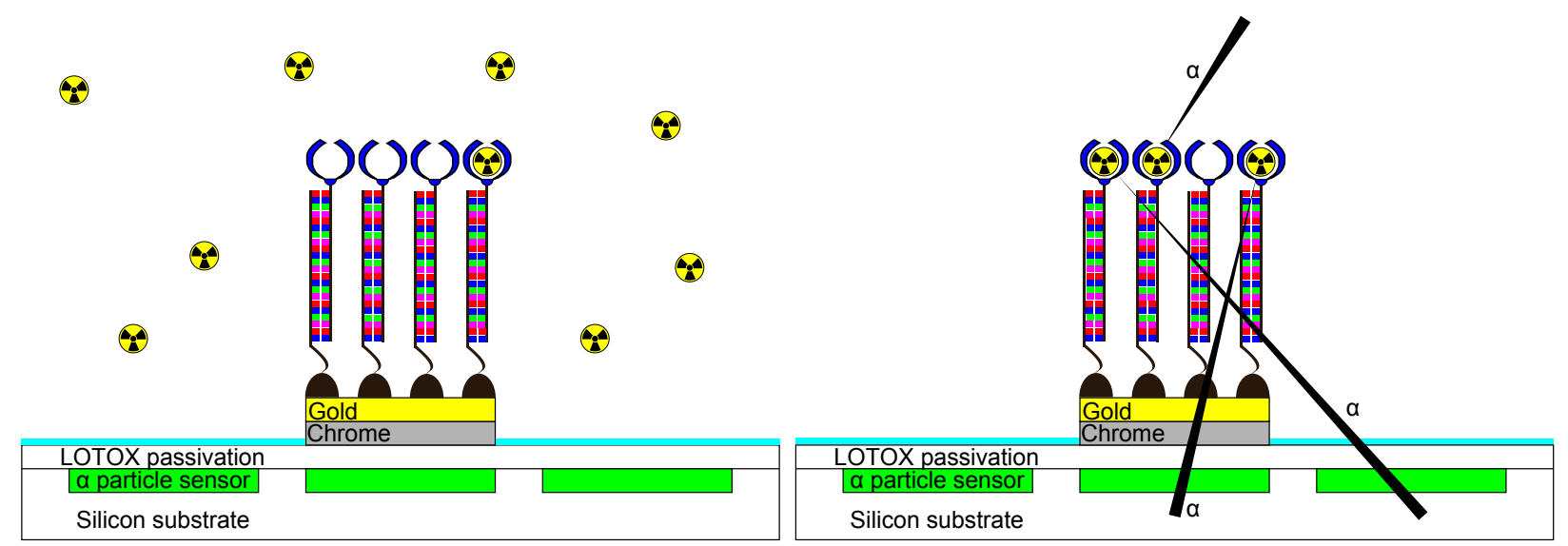

(c) Introduce and chelate radioisotope

(d) Rinse and measure $\alpha$ decay on $\alpha$ RAM surface

Figure 5.3: Pictorial representation of experimental procedure for chelating americium from solution. 
5. Apply thiolated DNA-chelator complex for 12 hours and rinse with ten $10 \mu \mathrm{L}$ applications of DI water.

6. Re-test the IC for a minimum of 24 hours.

7. Introduce ${ }^{241} \mathrm{Am}$ by placing a $10 \mu \mathrm{L}$ drop of $\mathrm{pH} 6$ buffered americium chloride solution on the surface of the IC for 2 hours at room temperature $\left(22^{\circ} \mathrm{C}\right)$.

8. Rinse the $\alpha \mathrm{RAM}$ five times with $10 \mu \mathrm{L}$ applications of DI water and dry.

9. Log and analyze data.

\subsection{Results and Discussion}

\subsubsection{Background Count Rate}

At each step during chemical processing the $\alpha$ RAM IC was tested for any change in performance. These measurements spanned a minimum of 24 hours and zero background events were detected. During handling however, two wirebonds were damaged resulting in data from rows 7 and 16 being absent from the trial with ${ }^{241} \mathrm{Am}$.

\subsubsection{Americium Chelation Experiment}

Figure 5.4 provides all the measurement results after chelating ${ }^{241} \mathrm{Am}$ from solution. Figure 5.4(a) presents the count rate observed from all the active cells on the IC as well as a least squares fit to the data. As is expected for a long lived $\alpha$ emitter like ${ }^{241} \mathrm{Am}$, the count rate remains constant throughout the measurement. Note that americium's progeny, neptunium-237 $\left({ }^{237} \mathrm{~Np}\right)$, is also an $\alpha$ emitter, but with a half life of 2.14 million years should not contribute significantly to the count rate observed. It should also be noted that the $\alpha$ emitters used in TAT have half-lives orders of 


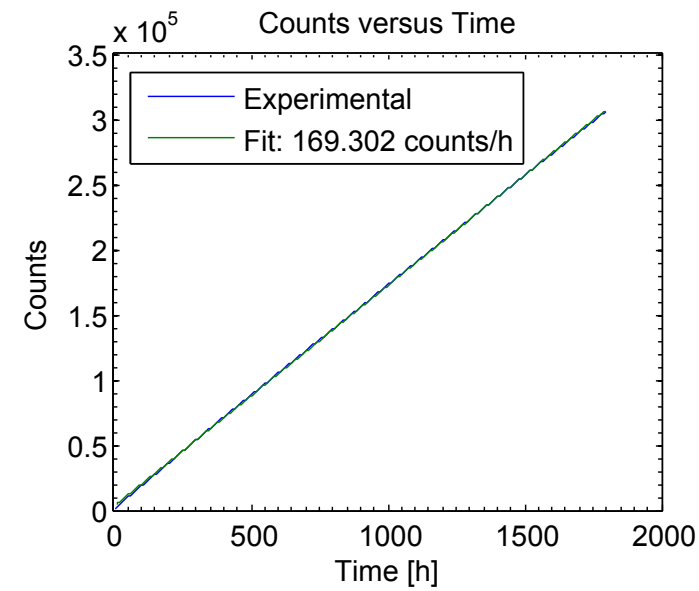

(a) Count rate for all functioning cells

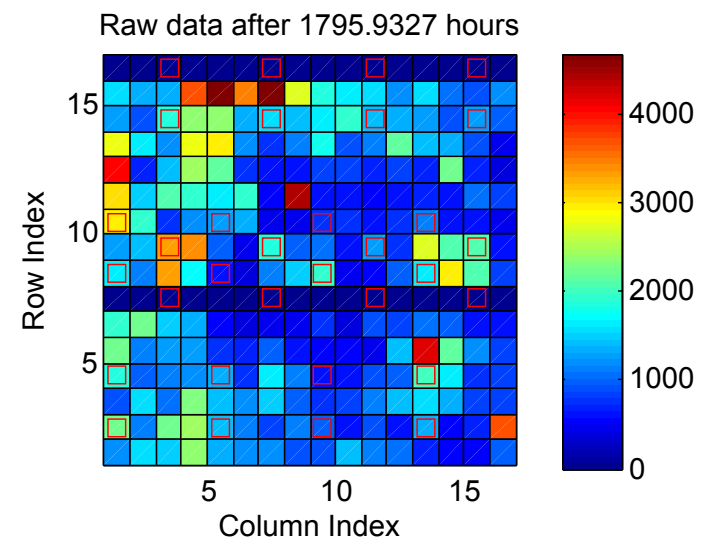

(c) Raw data with functionalized cells marked with a red square outline

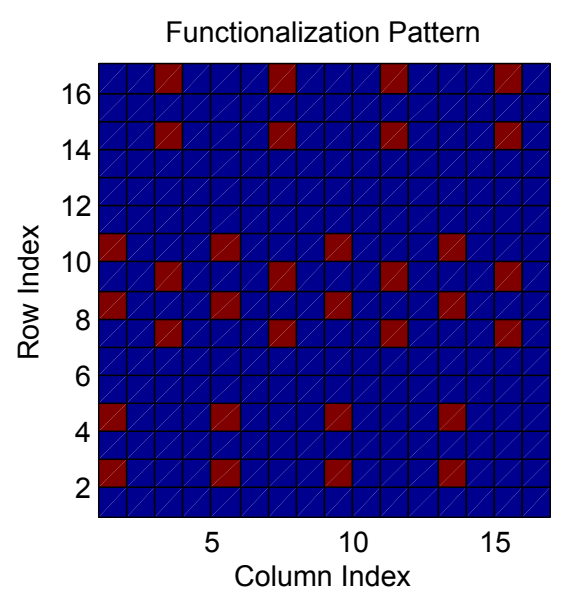

(b) Surface functionalization pattern. Dark red indicates a functionalized cell.

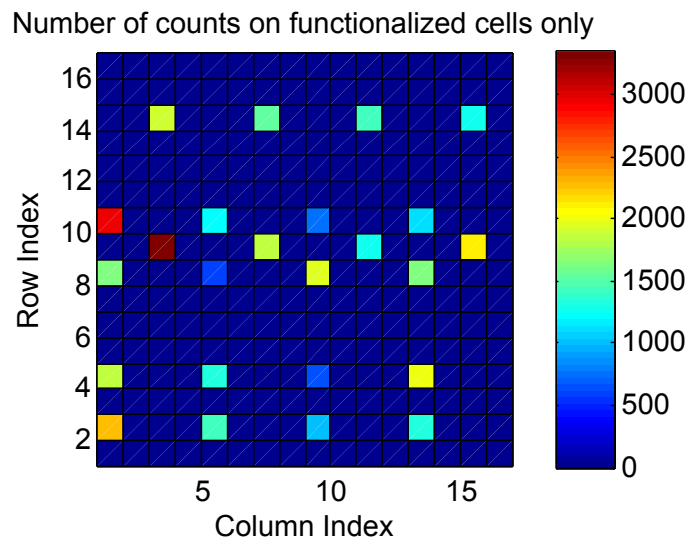

(d) Data on functionalized cells only

Figure 5.4: Experimental results after chelating ${ }^{241} \mathrm{Am}$ from solution.

magnitude less than ${ }^{241} \mathrm{Am}$ and thus better statistics will be acquired much faster when using the shorter lived TAT radioisotopes.

Figure 5.4(b) presents the pattern of chelator functionalized cells atop the $\alpha$ RAM IC, while Figure 5.4(c) presents raw counting data over the full surface of the IC. The raw data can be found in Appendix B. For ease of display Figure 5.4(d) shows the number of events recorded only by chelator functionalized cells. After 1796 hours, the 
mean number of counts across all unfunctionalized and functionalized cells were measured to be 1333 and 1608 counts, respectively. This corresponds to a $21 \%$ increase in the number of counts on functionalized cells.

As seen in Figure 5.4(c) there is an appreciable number of events observed on unfunctionalized cells. A number of factors may be responsible for this. The thiol chemistry used has a strong affinity to gold surfaces, but the thiolated DNA will also attach in lesser amounts to an oxide surface like the LOTOX passivation used on this chip. SigmaCote ${ }^{\circledR}$ was used to reduce the amount of non-specific surface attachment, but may not have been as effective as desired. Additionally the gold pattern deposited atop the IC should have been made more sparse with dimensions smaller than the cell size of the chip. The density of the current pattern, along with the potential for nonuniform thiolation, make observing the true location of the $\alpha$ emission difficult. A reduction in cell size, and a corresponding increase in the number of active cells could allow for more traditional image analysis to be performed on subsequent versions of the IC, or using a suitably modified commercial image sensor. Furthermore, and potentially most importantly, the protocol used in this demonstration could result in ${ }^{241} \mathrm{Am}$ plating out on the surface of the IC. DNA was used to tether the chelators to the surface of the IC, and thus experiments needed to be carried out at a $\mathrm{pH}$ which would not damage the DNA. A buffer was used to neutralize a small volume of the stock ${ }^{241} \mathrm{Am}$ solution to $\mathrm{pH} 6$ prior to chelation. Speciation plots of ${ }^{241} \mathrm{Am}$ at varying $\mathrm{pH}$ show that at increasing $\mathrm{pH}$, the americium will begin to precipitate [107-109]. Additional rinsing steps may also help reduce the amount of non-specifically bound chelator, as well as americium precipitate on the surface of the IC.

Observing Figure 5.4(c) shows a possible increase in activity near the edges of the IC. It is possible that while the americium solution on the IC surface during chelation lost volume due to evaporation, the fluid dynamics of the evaporating drop resulted in the area around the periphery of the IC experiencing a relative increase in americium 
concentration. Both functionalized and unfunctionalized would have been exposed to the increase in activity.

As this was a proof of concept experiment, only one $\alpha$ RAM IC was used in these

measurements. Improved statistics would be necessary in order to fully assess this, or other, experimental protocols. Improved selectivity could be had by optimizing the chemical protocols. Particularly, the chelation could be performed prior to introducing the $\alpha$ labelled biomolecules, or radioimmunoconjugates, onto the sensor surface.

Additionally, due to the low surface activity, and relatively short exposure, radiation induced sensor degradation is not expected to be a factor in these measurements. False counts due to continued radiation induced degradation would have introduce nonlinearity in the measured data.

\section{Calculation of Cell Activity}

A surface coverage $\left(N_{\text {surf }}\right)$ of $10^{12} \mathrm{~cm}^{-2}$ was expected for areas of the IC patterned in gold [84]. An estimate of the surface activity $\left(A_{\text {surf }}\right)$ is given in Equation 5.1 where $\lambda_{A m}$ is the lifetime of ${ }^{241} \mathrm{Am}$ and $S A$ is the surface area of a gold patterned square $\left(100 \times 100 \mu \mathrm{m}^{2}\right)$.

$$
A_{\text {surf }}=N_{\text {surf }} \cdot \lambda_{\text {Am }} \cdot S A
$$

This surface activity would correspond to $18 \alpha$ emissions per hour. Given that the IC will only detect $\alpha$ particles incident towards the substrate, only $50 \%$ of $\alpha$ emissions would be detected, so the expected count rate would be 9 counts/hour for a saturated surface. A mean count rate on functionalized cells of 0.9 counts/hour was observed. An explanation for the reduced counting rate observed could be non-uniform chelator attachment as well as our protocol not allowing the surface to become saturated. It 
should also be noted that the QE characterization carried out in [16] was for normally incident $\alpha$ particles, and it is possible that the QE is different for isotropic emissions on the IC's surface.

The experimental surface activity of each cell can be found simply using Equation 5.2 where $\tau_{\text {exp }}$ is the experimental count rate.

$$
A_{\text {experimental }}=\tau_{\text {exp }} \cdot 2 \cdot S A
$$

\subsubsection{Geant4 Modelling}

In order to validate the assumption that $50 \%$ of emissions are available for detection, and observe any geometry effects causing adjacent cells to be struck, a model of the $\alpha$ RAM was implemented using GEANT4 [14,15].

The three dimensional model consists of the $16 \times 16 \alpha$ RAM array with uniformly functionalized $100 \mu \mathrm{m}$ square cells. The column spacing is $46 \mu \mathrm{m}$, while the row spacing is either $10 \mu \mathrm{m}$ or $61 \mu \mathrm{m}$. Variation in row spacing can be seen in Figure 5.1(a). The sensor/passivation/Cr/Au is modelled as a silicon slab, while over the sensor is a layer of air. The thickness of the passivation $/ \mathrm{Cr} / \mathrm{Au}$ stack is $1.3 \mu \mathrm{m}$. The point at which $\alpha$ particles pass through the passivation and into the substrate is recorded and plotted. Tracks inside the silicon bulk were not followed, charged secondaries were suppressed during simulation, and "Standard EM" physics were used.

Simulation results with twenty million isotropically emitted $5.5 \mathrm{MeV} \alpha$ par-

ticles are presented in Figure 5.5. Figure 5.5(a) presents a 2D histogram of $\alpha$ particles passing into the $\alpha$ RAM bulk. Figure 5.5(b) presents a projection along $\mathrm{X}=0.27$ to $0.42 \mathrm{~mm}$ near the edge of a cell at $\mathrm{Y}=0.7 \mathrm{~mm}$ from Figure 5.5(a). Both the functionalized cell center, and edge of the nearest neighbour are shown.

Due to $\alpha$ particles following very linear trajectories, and being emitted so close to 

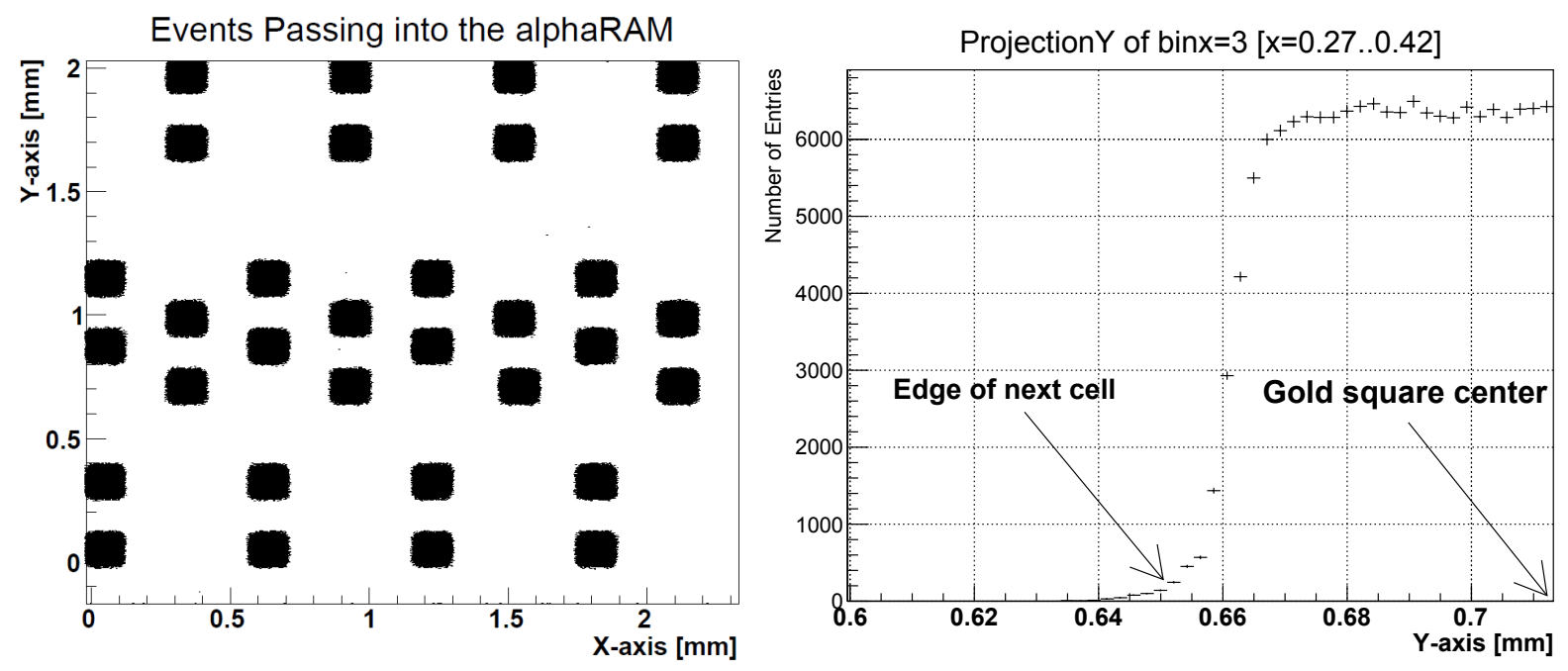

(a) 2D Histogram of events passing into the (b) Projection along $\mathrm{X}=0.27$ to $0.42 \mathrm{~mm}$ showing $\alpha$ RAM's active volume the edge of a functionalized gold square. The edge of the nearest cell is at $\mathrm{Y}=0.651 \mathrm{~mm}$.

Figure 5.5: GEANT4 simulation of $\alpha$ particle emissions on the $\alpha$ RAM surface.

the sensor surface, the majority of events emitted towards the sensor enter the cell atop which it was emitted.

The track length of a $5.5 \mathrm{MeV} \alpha$ particle in silicon should be on the order of $30 \mu \mathrm{m}$. Only in rare instances is it observed that a neighboring cell is struck by an $\alpha$ particle. This can be seen clearly in Figure 5.5(b) where only a few events are recorded in an adjacent cell. Note that charge diffusion in the bulk could still result in a neighboring cell being upset. Charge diffusion was not modelled in this simulation. Also, the assumption of uniform functionalization may not be true, and if the edges of the cells have a higher activity, the likelihood of a neighboring cell being upset increases.

This simulation also found that when 20 million events were simulated, 9,553,722 $\alpha$ particles entered into the silicon bulk. Thus $48 \%$ of events entered into the active volume of the sensor. Part of the $2 \%$ loss is due to cells at the periphery of the simulation volume being on the edge of the simulated surface, and losses in the passivation. Simulation supports that the $50 \%$ assumption was an acceptable simplification. 


\subsubsection{Continued Development}

In future versions of this IC, a more robust nitride encapsulation would be desirable. Additionally, eliminating the pinholes in the chrome/gold stack could allow the IC to be used dry without the need for the protective wax (See Figure 5.1(b)). Future experiments could also be carried out with the IC operating in solution provided appropriate packaging is used. As the radioisotopes are tethered at the sensor surface, the presence of a solution should not alter the sensor's detection efficiency. Encapsulation options could be as simple as additional wax, a polydimethylsiloxane (PDMS) reservoir, or a full microfluidics system.

The latest iteration of the $\alpha$ RAM IC has been submitted for fabrication using the AMS $0.35 \mu \mathrm{m}$ CMOS process. The design and layout of the IC was performed by a recent graduate of Carleton University's Electrical Engineering program, Alex J. Ross, under guidance from the author. Operation of the CMOS version of the $\alpha$ RAM is fundamentally the same as the nMOS version. Other than being implemented in CMOS, the significant change is the use of operational amplifiers, instead of sense amplifiers, in the determination of an $\alpha$ strike event. The chip remains a $16 \times 16$ array of $100 \mu \mathrm{m}$ square substrate diodes which are sequentially compared to a reference in order to determine if that cell has been struck by an $\alpha$ particle.

Modelling the effect of $\alpha$ particle induced tether damage is another avenue for exploration. Given the low activity of americium used, and the large number of chelating sites, $\alpha$ particle induced tether damage is not expected to be significant in this experiment.

The experiment presented here illustrates only one use of the testing platform. Many variations of experiments would be possible using this testbed and some examples include targeting agent characterization, and assessment of chelators or targeting agents under various biologically relevant conditions. This system could also be used 
in protocol development for drug synthesis by observing the effects of different buffers and temperatures during manufacture.

\subsection{Conclusion}

A metal-oxide-semiconductor (MOS) sensor has been designed, fabricated, and tested for use in the characterization of targeted alpha therapy (TAT) pharmaceuticals. The utility of the sensor as a test platform in the development of TAT pharmaceuticals was shown by functionalizing some cells with a popular chelator and observing the chelation of an $\alpha$ emitting actinide. A DNA tether was used to mimic an aptamer based TAT agent. Without any optimization of the chelation chemistry, a $21 \%$ increase of emissions was detected on cells functionalized with DOTA. The IC was able to quantitatively determine the amount of alpha emitter present over each cell, allowing the chelator and chelating chemistry to be assessed. This test platform could be used in the investigations of the specificity of the targeting agent, chelator retention ability, and TAT pharmaceutical yield, amongst others, all under varying experimental conditions. Future investigation appears to be warranted. 


\section{Chapter 6}

\section{Conclusions}

This thesis has advanced the state of the art in biomolecule detection by allowing for quantitative detection using radioactive labels whose decay is detected by a pixelated complementary metal-oxide-semiconductor (CMOS), or CMOS-compatible, sensor and demonstrates the usefulness of this technology as a testbed for radioimmunotherapy (RIT) pharmaceuticals.

A CMOS image sensor was used to detect the presence of radiolabelled target biomolecules captured on a functionalized surface. Using aptamer functionalization the system successfully detected phosphorus-32 labelled adenosine triphosphate (ATP) at a surface concentration of $2.3 \times 10^{7}$ molecules $/ \mathrm{cm}^{2}$, well below those typically associated with fluorescence-based sensor architectures. The system also demonstrated its amenability to multiplexed biomolecule detection. GEANT4, a Monte Carlo toolkit for simulating the passage of radiation through matter, was used to model the detection system. This system has applications in quantitative biomolecule detection and in the development of RIT pharmaceuticals employing $\beta^{-}$particle emitting isotopes.

Additionally, a CMOS compatible sensor has been designed, fabricated, and tested for use in the characterization of targeted alpha therapy (TAT) pharmaceuticals. The sensor consisted of a $16 \times 16$ array of $100 \mu \mathrm{m}$ square alpha particle sensitive cells 
and has been fabricated in-house using a simple nMOS process. A subset of the cells were functionalized for the attachment of chelators under investigation for new pharmaceuticals. As a demonstration of the utility of this sensor as a characterization platform, cells functionalized with 1,4,7,10-tetraazacyclododecane-1,4,7,10-tetraacetic acid (DOTA)-DNA conjugates were used to chelate americium-241 from solution, and the alpha particle emissions over the surface of the IC measured. The IC was able to quantitatively determine the amount of alpha emitter present over each cell, allowing the chelator and chelating chemistry to be assessed. Without any optimization of the chelation chemistry, a $21 \%$ increase of emissions was detected on cells functionalized with DOTA.

\subsection{Future Work}

Using Geant4, in conjunction with Sentaurus, it would be interesting to simulate application-specific detection systems. Factors including packaging, radioisotope emission spectrum, and device structures could all be varied in the search for an optimum application-specific detector design. Some applications may require higher spatial resolution, while others may require better charge collection. It would be interesting to optimize a sensor exclusively for radioimmunotherapy applications including both $\alpha$ and $\beta^{-}$emitting radioisotopes of interest. It would be particularly beneficial to use these simulation to determine whether a custom IC would be beneficial, or if current pixelated CMOS detectors are sufficient in their present state.

Continuing work on the testbed for targeted $\alpha$ therapy would require the development of improved encapsulation and packaging in order to have a sensor compatible with a wider array of chemistries and liquid testing environments. It would also be preferred to begin testing with the up-coming CMOS $\alpha$ RAM, or a suitable commercial IC. Testing various chelators and isotopes systems for chemical stability as well as 
optimum synthesis conditions would also be desirable. Modelling and observing the effect of $\alpha$ particle induced tether damage would be of interest. Additionally, characterization of the testbed over the range of temperatures used in radioimmunotherapy pharmaceutical formation would also be necessary. The same type of development would also be required for experimentation with $\beta^{-}$emitting radiopharmaceuticals.

Although not the intended application of this work, but due to the use of radiolabelled ATP in Chapter 4, it has been proposed that this technique could have applications in studying cellular response. In a number of interesting experiments measurement of ATP concentrations in the $\mu \mathrm{M}$ and nM range would be necessary [110-112]. In [110] it was observed that stimulating a particular cell resulted in nM concentrations of extracellular ATP being released. Another interesting example of how this technique could be used would be in the detection of cancer biomarkers. However, as the biomarkers themselves would not be radiolabelled, it would be necessary to develop a competitive assay for biomarker detection.

\subsection{Ultimate Detection Limit}

It is interesting to speculate on the ultimate sensitivity of the biomolecule detection technique introduced here. The lower limit of detection for both $\alpha$ and $\beta^{-}$detection systems would be determined by the amount of non-specific attachment to the surface of the sensor as well as the background "dark" count rate of the sensor itself. In the case of $\alpha$ particle sensors it is possible to make a sensor that has zero false counts for prolonged periods of time. In this case it is simply a matter of time until the measurement statistics will provide an acceptable amount of measurement error. While detecting $\beta^{-}$particles, the signals are much smaller and depending on the choice of counting threshold, a non-zero noise floor exists. At very low surface activity levels, the effect of cosmic rays must also be considered. A coarse estimate of 
the limit of detection for the $\beta^{-}$measurement system presented in this work using ${ }^{32} \mathrm{P}$ radiolabelled ATP would be $10^{7} \mathrm{~cm}^{-2}$. This assumes that 3 times the background count rate is required for detection. In the absence of non-specific attachment, the limit of detection becomes sensor limited and for the system here approaches $10^{5} \mathrm{~cm}^{-2}$ using the Canon 705S sensor with a 20\% threshold and 30 s exposures. Using shorter exposure times, but with the same total duration, would reduce the background count rate and would allow for the measurement of lower surface concentrations.

For a comparison, in a quantitative fluorescence assay performed in [113], the limit of quantification was found to be $1.3 \mathrm{pmol}$. For the measurements with ${ }^{32} \mathrm{P}$ labelled ATP, the aptamer coverage was expected to be on the order of $\mathrm{pmol} / \mathrm{cm}^{2}$ $\left(1 \times 10^{12} \mathrm{~cm}^{-2}\right)$, but the expected amount of bound ATP was calculated to be $6 \times 10^{9}$ molecules $/ \mathrm{cm}^{2}$. For $1 \mathrm{~mm}$ spots, the number of bound molecules would be $2 \times 10^{8}$ molecules, nearly 4 orders of magnitude lower than the fluorescence assay.

The most comparable luminescent technology for the TAT testbed would be "luminescent lathanides" [114]. Similarly to $\alpha$ based radioimmunoconjugates, a heavy element is chelated and a functionalizing molecule attached. In this case, the molecule attached acts as an antenna which absorbs and transfers energy to the lanthanide via luminescence resonance energy transfer (LRET). A typical lanthanide used is europium-III $\left(\mathrm{Eu}^{3+}\right)$. What makes this technology so appealing is that the lanthanide remains in an excited state much longer than typical fluorescent dyes. This allows the excitation source to be pulsed and measurements of the luminescent signal to be done while the excitation source is off, eliminating the fluorescence background from fast decaying sources. Very low detection limits have been reported with this method. In one case, $6.5 \mathrm{fmol} / \mathrm{L}$ was reported [114]. Variations on this technique with $\mathrm{Eu}^{3+}$ loaded polystyrene nanoparticles have claimed zeptomol sensitivity. Luminescent lanthanides have been used during the development of RIT pharmaceuticals, but this requires an assumption that the chemical properties of the radioisotope will be similar 
to the lanthanide used. The testbed proposed herein allows the radioimmunoconjugate to be observed directly.

Depending on the application, different radioisotopes, and possibly different sensors may be optimum. To reduce or eliminate charge spreading in the bulk, and improve spatial resolution a number of options exist and may include: thin epitaxial layers, different doping between epitaxial layer and substrate, and an IC developed on an SOI wafer. If charge collection is to be maximized, a thick lightly doped epitaxial layer would be advantageous. Additionally the detector could be developed in a high voltage CMOS process allowing much larger depletion regions thus improving the charge collection ability of the detector.

For higher energy $\beta^{-}$emitters, modern CMOS pixelated detectors are sufficient for quantitative detection as well as RIT pharmaceutical characterization. There may be some benefit to new detector design to optimize detection, but the benefits may not be sufficient to merit the cost of custom detector development when many low cost sensors already exist. The existence of low cost sensors does lend itself well to one-time-use sensors which would alleviate contamination concerns between experiments.

Although a custom nMOS IC was used for the TAT testbed experiment, a single commercial pixelated detector could be used in both $\alpha$ and $\beta^{-}$detection schemes. In [106] an inexpensive CMOS image sensor from a webcam was seen to be sensitive to both $\alpha$ and $\beta^{-}$particle radiation. If a commercial CMOS detector were to be used, developing a suitable patterning and encapsulation scheme, compatible with diced and/or packaged sensors, would be necessary. 


\subsection{Disseminations}

\section{$\beta^{-}$Experiments}

Results for the experiments using $\beta^{-}$emitting isotopes were presented and published at the IEEE Nuclear Science Symposium and Medical Imaging Conference (NSSMIC) in 2012 [12]. An expanded journal article was then prepared for, and published in, IEEE Transactions on Nuclear Science [13].

\section{$\alpha$ Experiments}

Results for the experiment using an $\alpha$ emitting isotope in the development of a TAT testbed have been prepared for, and accepted by, IEEE Sensors Journal [115].

\section{Previous and Related Experiments}

Development and characterization of the $\alpha$ RAM IC was presented, and published, prior to beginning Ph.D. studies in the proceedings of IEEE/CMC Microsystems and Nanoelectronics Research Conference (MNRC) 2008 [16] and IEEE Sensors 2008 [17]. This work continued and inspired two other papers published in the proceedings of IEEE Sensors 2011 [18] and SPIE Photonics North 2013 [106]. In [106] an optical image sensor was used to detect radon's $\alpha$ particle emitting progeny. Both [16] and [106] won best student paper awards. 


\section{List of References}

[1] M. W. Shinwari, M. J. Deen, and D. Landheer. "Study of the electrolyteinsulator-semiconductor field-effect transistor (EISFET) with applications in biosensor design." Microelectronics Reliability 47(12), 2025 - 2057. ISSN 00262714. Electronic system prognostics and health management Thermal, mechanical and multi-physics simulation and experiments in micro-electronics and micro-systems (EuroSimE 2006) (2007).

[2] M. A. Brun, K.-T. Tan, E. Nakata, M. J. Hinner, and K. Johnsson. "Semisynthetic fluorescent sensor proteins based on self-labeling protein tags." Journal of the American Chemical Society 131(16), 5873-5884. PMID: 19348459 (2009).

[3] J. Wang. "Nanoparticle-based electrochemical DNA detection." Analytica Chimica Acta 500(12), 247 - 257. ISSN 0003-2670. ANALYTICAL HORIZONS - An International Symposium celebrating the publication of Volume 500 of Analytica Chimica Acta (2003).

[4] C. Ding, H. Zhong, and S. Zhang. "Ultrasensitive flow injection chemiluminescence detection of DNA hybridization using nanocus tags." Biosensors and Bioelectronics 23(8), 1314 - 1318. ISSN 0956-5663 (2008).

[5] F. Qiu, D. Jiang, Y. Ding, J. Zhu, and L. Huang. "Monolayer-Barcoded Nanoparticles for On-Chip DNA Hybridization Assay." Angewandte Chemie International Edition 47(27), 5009-5012. ISSN 1521-3773 (2008).

[6] Y. C. Cao, R. Jin, and C. A. Mirkin. "Nanoparticles with Raman Spectroscopic Fingerprints for DNA and RNA Detection." Science 297(5586), 1536-1540 (2002).

[7] V. Weiler, M. Haase, C. Walter, W. Hoheisel, and H. Hummel. "Luminescent nanoparticles for molecular medicine." In "Society of Photo-Optical Instrumentation Engineers (SPIE) Conference Series," volume 6633 of Society of Photo-Optical Instrumentation Engineers (SPIE) Conference Series (2007). 
[8] C.-K. Lim, H.-J. Kim, H.-Y. Kim, Y. J. Kwon, S. W. Paek, and S. Y. Park. "DNA detection via programmed core-shell nanodot-assembly with concomitant fluorescence modulation." Journal of Photochemistry and Photobiology A: Chemistry 196(1), 94 - 98. ISSN 1010-6030 (2008).

[9] C. Hempen and U. Karst. "Labeling strategies for bioassays." Analytical and Bioanalytical Chemistry 384(3), 572-583. ISSN 1618-2642 (2006).

[10] E. Bertolucci, M. Conti, G. Mettivier, P. Russo, S. Amendolia, M. Bisogni, U. Bottigli, A. Ceccopieri, M. Ciocci, P. Delogu, M. Fantacci, P. Maestro, V. Marzulli, E. Pernigotti, N. Romeo, V. Rosso, A. Stefanini, and S. Stumbo. "GaAs pixel radiation detector as an autoradiography tool for genetic studies." Nuclear Instruments and Methods in Physics Research Section A: Accelerators, Spectrometers, Detectors and Associated Equipment 422(13), 242 - 246. ISSN 0168-9002 (1999).

[11] G. Mettivier, M. C. Montesi, and P. Russo. "First images of a digital autoradiography system based on a Medipix2 hybrid silicon pixel detector." Physics in Medicine and Biology 48(12), N173-N181 (2003).

[12] R. Griffin, O. Mozenson, M. Beking, M. DeRosa, G. Lopinski, and N. Tarr. "Quantitative radiolabeled biomolecule detection using a functionalized CMOS sensor." In "IEEE Nuclear Science Symposium and Medical Imaging Conference (NSS/MIC) 2012," pages 2048-2052. ISSN 1082-3654 (2012).

[13] R. H. Griffin, O. Mozenson, M. A. Beking, M. C. DeRosa, G. Lopinski, and N. G. Tarr. "Quantitative Radio-Labeled Biomolecule Detection Using a Functionalized CMOS Sensor." IEEE Transactions on Nuclear Science 61(3), 1112-1117. ISSN 0018-9499 (2014).

[14] J. Allison, K. Amako, J. Apostolakis, H. Araujo, P. Dubois, M. Asai, G. Barrand, R. Capra, S. Chauvie, R. Chytracek, G. Cirrone, G. Cooperman, G. Cosmo, G. Cuttone, G. Daquino, M. Donszelmann, M. Dressel, G. Folger, F. Foppiano, J. Generowicz, V. Grichine, S. Guatelli, P. Gumplinger, A. Heikkinen, I. Hrivnacova, A. Howard, S. Incerti, V. Ivanchenko, T. Johnson, F. Jones, T. Koi, R. Kokoulin, M. Kossov, H. Kurashige, V. Lara, S. Larsson, F. Lei, O. Link, F. Longo, M. Maire, A. Mantero, B. Mascialino, I. McLaren, P. Lorenzo, K. Minamimoto, K. Murakami, P. Nieminen, L. Pandola, S. Parlati, L. Peralta, J. Perl, A. Pfeiffer, M. Pia, A. Ribon, P. Rodrigues, G. Russo, S. Sadilov, G. Santin, T. Sasaki, D. Smith, N. Starkov, S. Tanaka, E. Tcherniaev, B. Tome, A. Trindade, P. Truscott, L. Urban, M. Verderi, A. Walkden, 
J. Wellisch, D. Williams, D. Wright, and H. Yoshida. "Geant4 developments and applications." IEEE Transactions on Nuclear Science 53(1), 270 -278. ISSN 0018-9499 (2006).

[15] S. Agostinelli, J. Allison, K. Amako, J. Apostolakis, H. Araujo, P. Arce, M. Asai, D. Axen, S. Banerjee, G. Barrand, F. Behner, L. Bellagamba, J. Boudreau, L. Broglia, A. Brunengo, H. Burkhardt, S. Chauvie, J. Chuma, R. Chytracek, G. Cooperman, G. Cosmo, P. Degtyarenko, A. Dell'Acqua, G. Depaola, D. Dietrich, R. Enami, A. Feliciello, C. Ferguson, H. Fesefeldt, G. Folger, F. Foppiano, A. Forti, S. Garelli, S. Giani, R. Giannitrapani, D. Gibin, J. J. G. Cadenas, I. Gonzlez, G. G. Abril, G. Greeniaus, W. Greiner, V. Grichine, A. Grossheim, S. Guatelli, P. Gumplinger, R. Hamatsu, K. Hashimoto, H. Hasui, A. Heikkinen, A. Howard, V. Ivanchenko, A. Johnson, F. W. Jones, J. Kallenbach, N. Kanaya, M. Kawabata, Y. Kawabata, M. Kawaguti, S. Kelner, P. Kent, A. Kimura, T. Kodama, R. Kokoulin, M. Kossov, H. Kurashige, E. Lamanna, T. Lampn, V. Lara, V. Lefebure, F. Lei, M. Liendl, W. Lockman, F. Longo, S. Magni, M. Maire, E. Medernach, K. Minamimoto, P. M. de Freitas, Y. Morita, K. Murakami, M. Nagamatu, R. Nartallo, P. Nieminen, T. Nishimura, K. Ohtsubo, M. Okamura, S. O’Neale, Y. Oohata, K. Paech, J. Perl, A. Pfeiffer, M. G. Pia, F. Ranjard, A. Rybin, S. Sadilov, E. D. Salvo, G. Santin, T. Sasaki, N. Savvas, Y. Sawada, S. Scherer, S. Sei, V. Sirotenko, D. Smith, N. Starkov, H. Stoecker, J. Sulkimo, M. Takahata, S. Tanaka, E. Tcherniaev, E. S. Tehrani, M. Tropeano, P. Truscott, H. Uno, L. Urban, P. Urban, M. Verderi, A. Walkden, W. Wander, H. Weber, J. P. Wellisch, T. Wenaus, D. C. Williams, D. Wright, T. Yamada, H. Yoshida, and D. Zschiesche. "Geant4-a simulation toolkit." Nuclear Instruments and Methods in Physics Research Section A: Accelerators, Spectrometers, Detectors and Associated Equipment 506(3), 250 - 303. ISSN 0168-9002 (2003).

[16] R. H. Griffin, H. Le, D. T. Jack, and N. G. Tarr. " $\alpha$ RAM: An $\alpha$ particle detecting MOS IC for radon monitoring." In "Microsystems and Nanoelectronics Research Conference 2008," pages 73-76. ISBN 978-1-4244-2920-2 (2008).

[17] R. H. Griffin, H. Le, D. T. Jack, A. Kochermin, and N. G. Tarr. "Radon monitor using custom $\alpha$-detecting MOS IC." In "IEEE Sensors 2008," pages 906-909. ISSN 1930-0395 (2008).

[18] R. H. Griffin, A. Kochermin, N. G. Tarr, H. McIntosh, H. Ding, J. Weber, and R. Falcomer. "Sensitive, fast-responding passive electrostatic radon monitor." In "IEEE Sensors 2011," pages 1074-1077. ISSN 1930-0395 (2011). 
[19] G. Knoll. Radiation Detection and Mesurement, 2nd Ed. John Wiley \& Sons, New York, NY. ISBN 0-471-81504-7 (1989).

[20] R. D. Evans and A. Noyau. The Atomic Nucleus. McGraw-Hill, New York (1955).

[21] J. F. Ziegler, J. P. Biersack, and M. D. Ziegler. SRIM: The Stopping and Range of Ions in Matter. Lulu Press Co., Morrisville, NC (2008).

[22] L. D. Landau. "On the energy loss of fast particles by ionization." J. Phys. 8, 201-205 (1944).

[23] S. Meroli. Silicon pixel detectors for high precision measurements. Ph.D. thesis, University of Perugia, Italy (2011).

[24] S. Hancock, F. James, J. Movchet, P. G. Rancoita, and L. VanRossum. "Energy loss and energy straggling of protons and pions in the momentum range 0.7 to 115 GeV/c." Phys. Rev. A 28, 615-620 (1983).

[25] P. Vavilov. "Ionization losses of high-energy heavy particles." Soviet Phys. JETP 5 (1957).

[26] O. Blunck and S. Leisegang. "Zum energieverlust schneller elektronen in dünnen schichten." Zeitschrift für Physik 128(4), 500-505 (1950).

[27] A. P. Shulek, B. Golovin, L. Kulyukin, S. Medved, and P. Pavolvic. "Fluctuations of ionization loss." Soviet Journal of Nuclean Physics-USSR 4(3), 400 (1967).

[28] H. Bichsel. "Straggling of Heavy Charged Particles: Comparison of Born Hydrogenic-Wave-Function Approximation with Free-Electron Approximation." Phys. Rev. B 1, 2854-2862 (1970).

[29] H. Esbensen, O. Fich, J. A. Golovchenko, S. Madsen, H. Nielsen, H. E. Schiøtt, E. Uggerhøj, C. Vraast-Thomsen, G. Charpak, S. Majewski, G. Odyniec, G. Petersen, F. Sauli, J. P. Ponpon, and P. Siffert. "Random and channeled energy loss in thin germanium and silicon crystals for positive and negative $2-15-\mathrm{GeV} / \mathrm{c}$ pions, kaons, and protons." Phys. Rev. B 18, 1039-1054 (1978).

[30] S. Kannengießer. Optimization of the Synthesis of Ac-225-labelled DOTARadioimmunoconjugates for Targeted Alpha Therapy, based on Investigations on the Complexation of Trivalent Actinides by DOTA. Ph.D. thesis, Universität Heidelberg, Germany (2013). 
[31] C. F. Qu, E. Y. Song, Y. Li, S. M. A. Rizvi, C. Raja, R. Smith, A. Morgenstern, C. Apostolidis, and B. J. Allen. "Pre-clinical Study of ${ }^{213}$ Bi Labeled PAI2 for the Control of Micrometastatic Pancreatic Cancer." Clinical \& Experimental Metastasis 22(7), 575-586. ISSN 0262-0898 (2005).

[32] S. Vispé and M. S. Satoh. "DNA Repair Patch-mediated Double Strand DNA Break Formation in Human Cells." Journal of Biological Chemistry 275(35), 27386-27392 (2000).

[33] M. A. Ritter, J. E. Cleaver, and C. A. Tobias. "High-LET radiations induce a large proportion of non-rejoining DNA breaks." (266), 653-655 (1977).

[34] B. J. Allen, C. Raja, S. Rizvi, Y. Li, W. Tsui, P. Graham, J. F. Thompson, R. A. Reisfeld, J. Kearsley, A. Morgenstern, and C. Apostolidis. "Clinical Trial Intralesional Targeted Alpha Therapy for Metastatic Melanoma." Cancer biology $\&$ therapy 4(12), 1318-1324 (2005).

[35] D. Drouin, A. R. Couture, D. Joly, X. Tastet, V. Aimez, and R. Gauvin. "CASINO V2.42A Fast and Easy-to-use Modeling Tool for Scanning Electron Microscopy and Microanalysis Users." Scanning 29(3), 92-101. ISSN 1932-8745 (2007).

[36] H. Demers, N. Poirier-Demers, A. R. Couture, D. Joly, M. Guilmain, N. de Jonge, and D. Drouin. "Three-dimensional electron microscopy simulation with the CASINO Monte Carlo software." Scanning 33(3), 135-146. ISSN 1932-8745 (2011).

[37] R. Forster and T. Godfrey. "MCNP - a general Monte Carlo code for neutron and photon transport." In "Monte-Carlo Methods and Applications in Neutronics, Photonics and Statistical Physics," volume 240 of Lecture Notes in Physics, pages 33-55. Springer Berlin Heidelberg. ISBN 978-3-540-16070-0 (1985).

[38] F. Salvat, J. M. Fernández-Varea, and J. Sempau. "PENELOPE-2008: A code system for Monte Carlo simulation of electron and photon transport." In "NEAOECD Workshop Proceedings 2008," (2009).

[39] I. Kawrakow and D. Rogers. "The EGSnrc code system: Monte Carlo simulation of electron and photon transport." National Research Council of Canada Report PIRS-701 (2003).

[40] Synopsys. Taurus Medici: Medici User Guide Version I-2013.12. Synopsys Incorporated, Mountain View, CA (2013). 
[41] Synopsys. Sentaurus Device User Guide Version J-2014.09. Synopsys Incorporated, Mountain View, CA (2014).

[42] G. Charpak, D. Rahm, and H. Steiner. "Some developments in the operation of multiwire proportional chambers." Nuclear Instruments and Methods 80(1), 13 - 34. ISSN 0029-554X (1970).

[43] S. M. Sze and K. K. Ng. Physics of semiconductor devices, 3rd Edition. John Wiley \& Sons. ISBN 978-0-471-14323-9 (2006).

[44] S. M. Sze and M.-K. Lee. Semiconductor Devices: Physics and Technology, 3rd Edition. John Wiley \& Sons. ISBN 978-0-470-53794-7 (2012).

[45] Y. Tsividis and C. McAndrew. Operation and Modeling of the MOS Transistor, Third Edition. Oxford University Press. ISBN 978-0-195-17015-3 (2010).

[46] A. S. Grove. Physics and technology of semiconductor devices. John Wiley \& Sons, New York. ISBN 978-0-471-32998-5 (1967).

[47] J. Cabello. High throughput digital $\beta$ autoradiography imaging. Ph.D. thesis, University of Surrey, Guildford, Surrey, U.K. (2009).

[48] E. Heckathorne, L. Tiefer, F. Daghighian, and M. Dahlbom. "Evaluation of arrays of silicon photomultipliers for beta imaging." In "IEEE Nuclear Science Symposium Conference Record 2008," pages 1626-1631. ISSN 1095-7863 (2008).

[49] C. Cappellini, A. Bulgheroni, M. Caccia, V. Chmill, M. Jastrzab, F. Risigo, and P. Scopelliti. "Imaging of biological samples with silicon pixel detectors." Nuclear Instruments and Methods in Physics Research Section A: Accelerators, Spectrometers, Detectors and Associated Equipment 591(1), 34 - 37. ISSN 0168-9002 (2008).

[50] R. Turchetta, J. Berst, B. Casadei, G. Claus, C. Colledani, W. Dulinski, Y. Hu, D. Husson, J. L. Normand, J. Riester, G. Deptuch, U. Goerlach, S. Higueret, and M. Winter. "A monolithic active pixel sensor for charged particle tracking and imaging using standard VLSI CMOS technology." Nuclear Instruments and Methods in Physics Research Section A: Accelerators, Spectrometers, Detectors and Associated Equipment 458(3), 677 - 689. ISSN 0168-9002 (2001).

[51] G. Deptuch. "Tritium autoradiography with thinned and back-side illuminated monolithic active pixel sensor device." Nuclear Instruments and Methods in Physics Research Section A: Accelerators, Spectrometers, Detectors and Associated Equipment 543(23), 537 - 548. ISSN 0168-9002 (2005). 
[52] A. Bulgheroni, M. Bianda, M. Caccia, C. Cappellini, A. Mozzanica, R. Ramelli, and F. Risigo. "Characterization of a thinned back illuminated MIMOSA V sensor as a visible light camera." Nuclear Instruments and Methods in Physics Research Section A: Accelerators, Spectrometers, Detectors and Associated Equipment 565(1), 221 - 226. ISSN 0168-9002 (2006).

[53] J. Matheson, G. Moldovan, A. Clark, M. Prydderch, R. Turchetta, G. Derbyshire, A. Kirkland, and N. Allinson. "Characterisation of a monolithic active pixel sensor for electron detection in the energy range 10-20 keV." Nuclear Instruments and Methods in Physics Research Section A: Accelerators, Spectrometers, Detectors and Associated Equipment 608(1), 199 - 205. ISSN 0168-9002 (2009).

[54] I. Perić. "A novel monolithic pixelated particle detector implemented in highvoltage CMOS technology." Nuclear Instruments and Methods in Physics Research Section A: Accelerators, Spectrometers, Detectors and Associated Equipment 582(3), 876 - 885. ISSN 0168-9002 (2007).

[55] Y. Arai, M. Hazumi, Y. Ikegami, T. Kohriki, O. Tajima, S. Terada, T. Tsuboyama, Y. Unno, H. Ushiroda, H. Ikeda, K. Hara, H. Ishino, T. Kawasaki, E. Martin, G. Varner, H. Tajima, M. Ohno, K. Fukuda, H. Komatsubara, J. Ida, and H. Hayashi. "Monolithic Pixel Detector in a $0.15 \mu \mathrm{m}$ SOI Technology." In "IEEE Nuclear Science Symposium Conference Record, 2006," volume 3, pages 1440-1444. ISSN 1095-7863 (2006).

[56] N. Wyrsch, S. Dunand, C. Miazza, A. Shah, G. Anelli, M. Despeisse, A. Garrigos, P. Jarron, J. Kaplon, D. Moraes, S. C. Commichau, G. Dissertori, and G. M. Viertel. "Thin-film silicon detectors for particle detection." Physica Status Solidi (c) 1(5), 1284-1291. ISSN 1610-1642 (2004).

[57] F. Hartmann. Evolution of Silicon Sensor Technology in Particle Physics. Springer. ISBN 978-3-540-44774-0 (2009).

[58] W. S. Boyle and G. E. Smith. "Charge Coupled Semiconductor Devices." Bell System Technical Journal 49(4), 587-593. ISSN 1538-7305 (1970).

[59] S. Mendis and E. R. Fossum. CMOS active pixel image sensor. NASA Jet Propulsion Laboratory, California Institute of Technology, Pasadena, CA (1993). 
[60] E. R. Fossum. "Active pixel sensors: are CCDs dinosaurs?" In "Proceedings of SPIE Charge-Coupled Devices and Solid State Optical Sensors III," volume 1900, pages 2-14 (1993).

[61] D. Litwiller. "CCD vs. CMOS." Photonics Spectra (2002).

[62] D. Litwiller. "CMOS vs. CCD: Maturing Technologies, Maturing Markets." Photonics Spectra (2005).

[63] B. Carlson. "Comparison of modern CCD and CMOS image sensor technologies and systems for low resolution imaging." In "Proceedings of IEEE Sensors 2002," volume 1, pages 171-176 (2002).

[64] ON Semiconductor. KAF-1001 Image Sensor Datasheet (2014).

[65] M. Moll. Radiation damage in silicon particle detectors. Ph.D. thesis, Universität Hamburg, Germany (1999).

[66] G. Lindström. "Radiation damage in silicon detectors." Nuclear Instruments and Methods in Physics Research Section A: Accelerators, Spectrometers, Detectors and Associated Equipment 512(12), 30 - 43. ISSN 0168-9002. Proceedings of the 9th European Symposium on Semiconductor Detectors: New Developments on Radiation Detectors (2003).

[67] P. Jerram, D. Burt, N. Guyatt, V. Hibon, J. Vaillant, and Y. Henrion. "Backthinned cmos sensor optimization." In "Proc. SPIE," volume 7598, pages 759813-1-759813-12 (2010).

[68] J. Jakubek. "Data processing and image reconstruction methods for pixel detectors." Nuclear Instruments and Methods in Physics Research Section A: Accelerators, Spectrometers, Detectors and Associated Equipment 576(1), 223 - 234. ISSN 0168-9002 (2007).

[69] R. Huber, C. Seidl, E. Schmid, S. Seidenschwang, K.-F. Becker, C. Schuhmacher, C. Apostolidis, T. Nikula, E. Kremmer, M. Schwaiger, and R. Senekowitsch-Schmidtke. "Locoregional $\alpha$-radioimmunotherapy of intraperitoneal tumor cell dissemination using a tumor-specific monoclonal antibody." Clinical cancer research 9(10), 3922s-3928s (2003).

[70] A. I. Kassis and S. J. Adelstein. "Radiobiologic principles in radionuclide therapy." Journal of Nuclear Medicine 46(1 suppl), 4S-12S (2005). 
[71] L. Li, W. Wang, X. Xu, H. Wang, S. Liao, W. Li, W. Zhang, D. Liu, B. Cao, S. Wang, K. Shen, and D. Ma. "Aptamer-based radioimmunotherapy: the feasibility and prospect in cancer therapy." Journal of Radioanalytical and Nuclear Chemistry 290(2), 453-457. ISSN 0236-5731 (2011).

[72] D. S. Wilbur, D. K. Hamlin, M.-K. Chyan, and M. W. Brechbiel. "Streptavidin in Antibody Pretargeting. 5. Chemical Modification of Recombinant Streptavidin for Labeling with the $\alpha$-Particle-Emitting Radionuclides ${ }^{213} \mathrm{Bi}$ and ${ }^{211}$ At." Bioconjugate Chemistry 19(1), 158-170. PMID: 18072725 (2008).

[73] S. Srivastava and E. Dadachova. "Recent advances in radionuclide therapy." Seminars in Nuclear Medicine 31(4), 330 - 341. ISSN 0001-2998 (2001).

[74] I. Davis, K. Glowienka, R. Boll, K. Deal, M. Brechbiel, M. Stabin, P. Bochsler, S. Mirzadeh, and S. Kennel. "Comparison of ${ }^{225}$ actinium chelates: tissue distribution and radiotoxicity." Nuclear Medicine and Biology 26(5), 581 - 589. ISSN 0969-8051 (1999).

[75] M. R. McDevitt, D. Ma, J. Simon, R. Frank, and D. A. Scheinberg. "Design and synthesis of ${ }^{225}$ Ac radioimmunopharmaceuticals." Applied Radiation and Isotopes 57(6), 841 - 847. ISSN 0969-8043 (2002).

[76] L. L. Chappell, K. A. Deal, E. Dadachova, and M. W. Brechbiel. "Synthesis, Conjugation, and Radiolabeling of a Novel Bifunctional Chelating Agent for ${ }^{225}$ Ac Radioimmunotherapy Applications." Bioconjugate Chemistry 11(4), 510519. PMID: 10898572 (2000).

[77] M. Miederer, D. A. Scheinberg, and M. R. McDevitt. "Realizing the potential of the Actinium-225 radionuclide generator in targeted alpha particle therapy applications." Advanced Drug Delivery Reviews 60(12), 1371 - 1382. ISSN 0169-409X. Delivery Systems for the Targeted Radiotherapy of Cancer (2008).

[78] M. R. McDevitt, G. Sgouros, R. D. Finn, J. L. Humm, J. G. Jurcic, S. M. Larson, and D. A. Scheinberg. "Radioimmunotherapy with alpha-emitting nuclides." European Journal of Nuclear Medicine 25(9), 1341-1351. ISSN 03406997 (1998).

[79] C. Fan and G. Lopinski. "STM and HREELS investigation of gas phase silanization on hydroxylated Si(100)." Surface Science 604(1112), 996 - 1001. ISSN 0039-6028 (2010). 
[80] Y. Li, O. Mozenson, D. Landheer, and G. Lopinski. "Stable Biomolecule Attachment to Thermal Silicon Oxide Surfaces." Unpulished (2012).

[81] S. O. Kelley, J. K. Barton, N. M. Jackson, and M. G. Hill. "Electrochemistry of Methylene Blue Bound to a DNA-Modified Electrode." Bioconjugate Chemistry 8(1), 31-37 (1997).

[82] H. Hong, Y. Zhang, J. Sun, and W. Cai. "Molecular imaging and therapy of cancer with radiolabeled nanoparticles." Nano Today 4(5), 399 - 413. ISSN 1748-0132 (2009).

[83] G. Kramer-Marek and J. Capala. "The role of nuclear medicine in modern therapy of cancer." Tumor Biology 33(3), 629-640. ISSN 1010-4283 (2012).

[84] E. M. Boon, J. E. Salas, and J. K. Barton. "An electrical probe of protein-DNA interactions on DNA-modified surfaces." Nature Biotechnology 20, 282-286 (2002).

[85] S. R. Wasserman, Y. T. Tao, and G. M. Whitesides. "Structure and reactivity of alkylsiloxane monolayers formed by reaction of alkyltrichlorosilanes on silicon substrates." Langmuir 5(4), 1074-1087 (1989).

[86] J. B. Lamture, K. LBeattie, B. E. Burke, M. D. Eggers, D. J. Ehrlich, R. Fowler, M. A. Hollis, B. B. Kosicki, R. K. Reich, S. R. Smith, R. S. Varma, and M. E. Hogan. "Direct detection of nucleic acid hybridization on the surface of a charge coupled device." Nucleic Acids Research 22(11), 2121-2125 (1994).

[87] M. Esposito, G. Mettivier, and P. Russo. " ${ }^{14} \mathrm{C}$ autoradiography with an energysensitive silicon pixel detector." Physics in Medicine and Biology 56(7), 19471965 (2011).

[88] J. Cabello and K. Wells. "The spatial resolution of silicon based electron detectors in $\beta$-autoradiography." Physics in Medicine and Biology 55(6), 1677-1699 (2010).

[89] J. Cabello, A. Bailey, I. Kitchen, M. Prydderch, A. Clark, R. Turchetta, and K. Wells. "Digital autoradiography using room temperature ccd and cmos imaging technology." Physics in Medicine and Biology 52(16), 4993-5011 (2007).

[90] G. Cirrone, G. Cuttone, F. D. Rosa, L. Pandola, F. Romano, and Q. Zhang. "Validation of the geant4 electromagnetic photon cross-sections for elements and compounds." Nuclear Instruments and Methods in Physics Research 
Section A: Accelerators, Spectrometers, Detectors and Associated Equipment 618(1-3), 315 - 322. ISSN 0168-9002 (2010).

[91] J. Sempau, J. Fernández-Varea, E. Acosta, and F. Salvat. "Experimental benchmarks of the Monte Carlo code PENELOPE." Nuclear Instruments and Methods in Physics Research Section B: Beam Interactions with Materials and Atoms 207(2), 107 - 123. ISSN 0168-583X (2003).

[92] S. Incerti, A. Ivanchenko, M. Karamitros, A. Mantero, P. Moretto, H. N. Tran, B. Mascialino, C. Champion, V. N. Ivanchenko, M. A. Bernal, Z. Francis, C. Villagrasa, G. Baldacchino, P. Guye, R. Capra, P. Nieminen, and C. Zacharatou. "Comparison of GEANT4 very low energy cross section models with experimental data in water." Medical Physics 37(9), 4692-4708 (2010).

[93] M. Raine, A. Valentin, M. Gaillardin, and P. Paillet. "Improved Simulation of Ion Track Structures Using New Geant4 Models - Impact on the Modeling of Advanced Technologies Response." IEEE Transactions on Nuclear Science 59(6), 2697-2703. ISSN 0018-9499 (2012).

[94] A. Valentin, M. Raine, J.-E. Sauvestre, M. Gaillardin, and P. Paillet. "Geant4 physics processes for microdosimetry simulation: Very low energy electromagnetic models for electrons in silicon." Nuclear Instruments and Methods in Physics Research Section B: Beam Interactions with Materials and Atoms 288, 66 - 73. ISSN 0168-583X (2012).

[95] M. J. Berger. ESTAR: Stopping-power and range tables for electrons. NIST, Gaithersburg, MD (2002).

[96] M. Murat, A. Akkerman, and J. Barak. "Electron and ion tracks in silicon: Spatial and temporal evolution." IEEE Transactions on Nuclear Science 55(6), 3046-3054. ISSN 0018-9499 (2008).

[97] M. J. Berger. ASTAR: Stopping-power and range tables for helium ions. NIST, Gaithersburg, MD (1999).

[98] R. Firestone, V. Shirley, C. Baglin, S. Chu, and J. Zipkin. Table of Isotopes, 8-th Edition. John Willey \& Sons, New York. ISBN 978-0-471-24699-2 (1998).

[99] Chipworks. Imager Process Review of the Canon EOS 350D 8Mp (705S) CMOS Image Sensor. Chipworks Inc., Ottawa, Canada. IPR-0511-803 (1998).

[100] R. N. Clark. "Digital Camera Reviews and Sensor Performance Summary." (2014). 
[101] O. C. Allkofer, K. Carstensen, W. D. Dau, and H. Jokisch. "The absolute cosmic ray flux at sea level." Journal of Physics G: Nuclear Physics 1(6), L51 (1975).

[102] H. Urata, K. Nomura, S. Wada, and M. Akagi. "Fluorescent-labeled singlestrand ATP aptamer DNA: Chemo- and enantio-selectivity in sensing adenosine." Biochemical and Biophysical Research Communications 360(2), 459 463. ISSN 0006-291X (2007).

[103] Z. Tang, P. Mallikaratchy, R. Yang, Y. Kim, Z. Zhu, H. Wang, and W. Tan. "Aptamer Switch Probe Based on Intramolecular Displacement." Journal of the American Chemical Society 130(34), 11268-11269. PMID: 18680291 (2008).

[104] C. Witte and F. Lisdat. "Direct Detection of DNA and DNA-Ligand Interaction by Impedance Spectroscopy." Electroanalysis 23(2), 339-346. ISSN 1521-4109 (2011).

[105] D. E. Huizenga and J. W. Szostak. "A DNA Aptamer That Binds Adenosine and ATP." Biochemistry 34(2), 656-665. PMID: 7819261 (1995).

[106] R. H. Griffin and N. G. Tarr. "Optical image sensors and their application in radon detection." In "Proc. SPIE," volume 8915, pages 89151C-89151C-7 (2013).

[107] R. Byrne, L. Kump, and K. Cantrell. "The influence of temperature and pH on trace metal speciation in seawater." Marine Chemistry 25(2), 163 - 181. ISSN 0304-4203 (1988).

[108] B. Allard, V. Moulin, L. Basso, M. Tran, and D. Stammose. "Americium adsorption on alumina in the presence of humic materials." Geoderma 44(23), 181 - 187. ISSN 0016-7061 (1989).

[109] N. Takeno. "Atlas of Eh-pH diagrams." Technical report, National Institute of Advanced Industrial Science and Technology. Geological survey of Japan open file report 419 (2005).

[110] G. G. Yegutkin, A. Mikhailov, S. S. Samburski, and S. Jalkanen. "The Detection of Micromolar Pericellular ATP Pool on Lymphocyte Surface by Using Lymphoid Ecto-Adenylate Kinase as Intrinsic ATP Sensor." Molecular Biology of the Cell 17(8), 3378-3385 (2006). 
[111] B. Kalthof, M. Bechem, K. Flocke, L. Pott, and M. Schramm. "Kinetics of ATP-induced $\mathrm{Ca} 2+$ transients in cultured pig aortic smooth muscle cells depend on ATP concentration and stored Ca2+." The Journal of Physiology 466(1), 245-262 (1993).

[112] A. Hazama, S. Hayashi, and Y. Okada. "Cell surface measurements of ATP release from single pancreatic $\beta$ cells using a novel biosensor technique." Pflügers Archiv 437(1), 31-35. ISSN 0031-6768 (1998).

[113] P. M. Wilson, M. J. LaBonte, J. Russell, S. Louie, A. A. Ghobrial, and R. D. Ladner. "A novel fluorescence-based assay for the rapid detection and quantification of cellular deoxyribonucleoside triphosphates." Nucleic Acids Research 39(17), e112 (2011).

[114] A. Hagan and T. Zuchner. "Lanthanide-based time-resolved luminescence immunoassays." Analytical and Bioanalytical Chemistry 400(9), 2847-2864. ISSN 1618-2642 (2011).

[115] R. H. Griffin, E. M. McConnell, M. C. DeRosa, and N. G. Tarr. "MOS testbed for the characterization of targeted alpha therapy pharmaceuticals." IEEE Sensors Journal ISSN 1530-437X. Accepted (2014). 


\section{Appendix A}

\section{Sentaurus Input Files}

\section{A.1 2D Substrate Diode Structure}

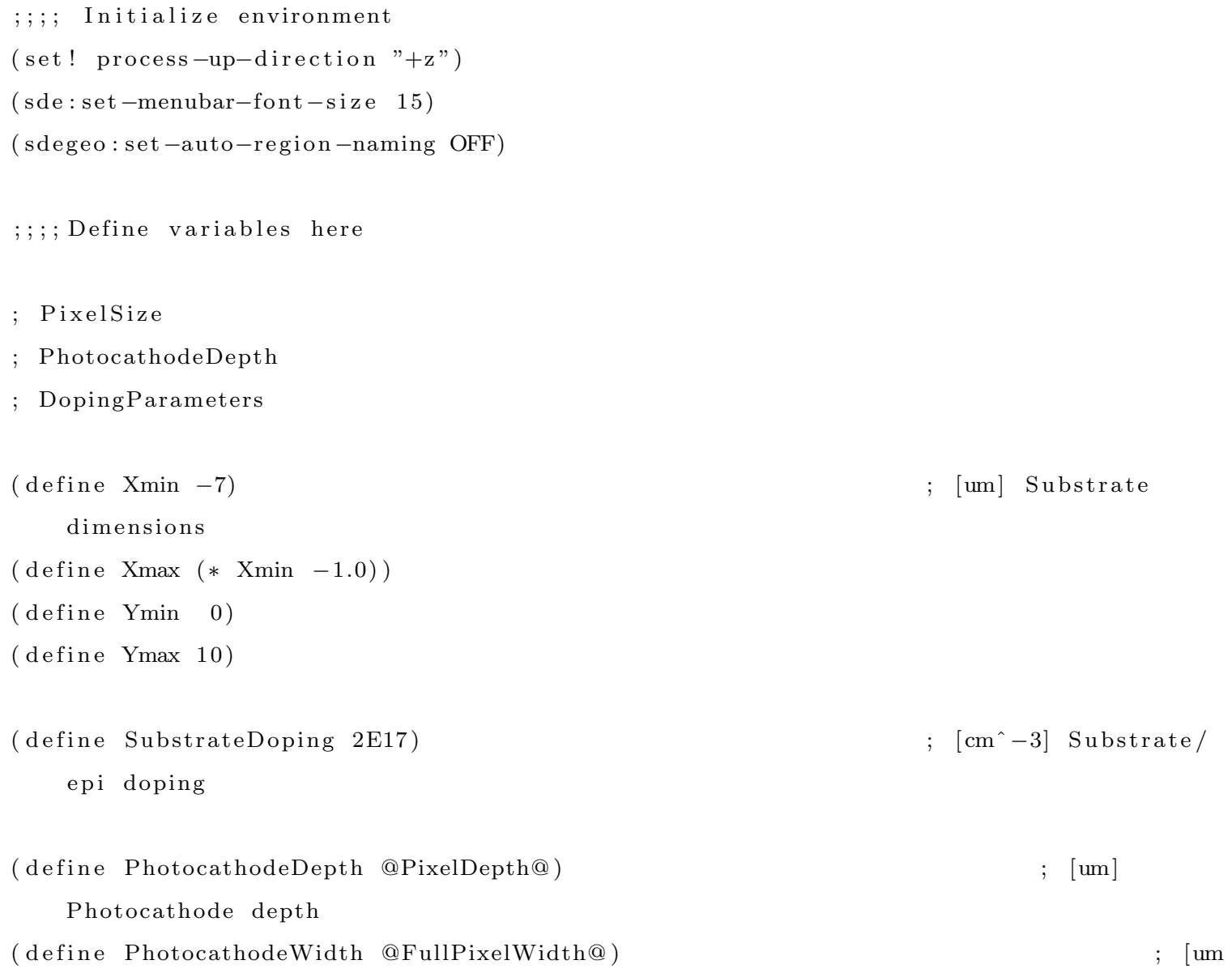

; [um] Substrate 


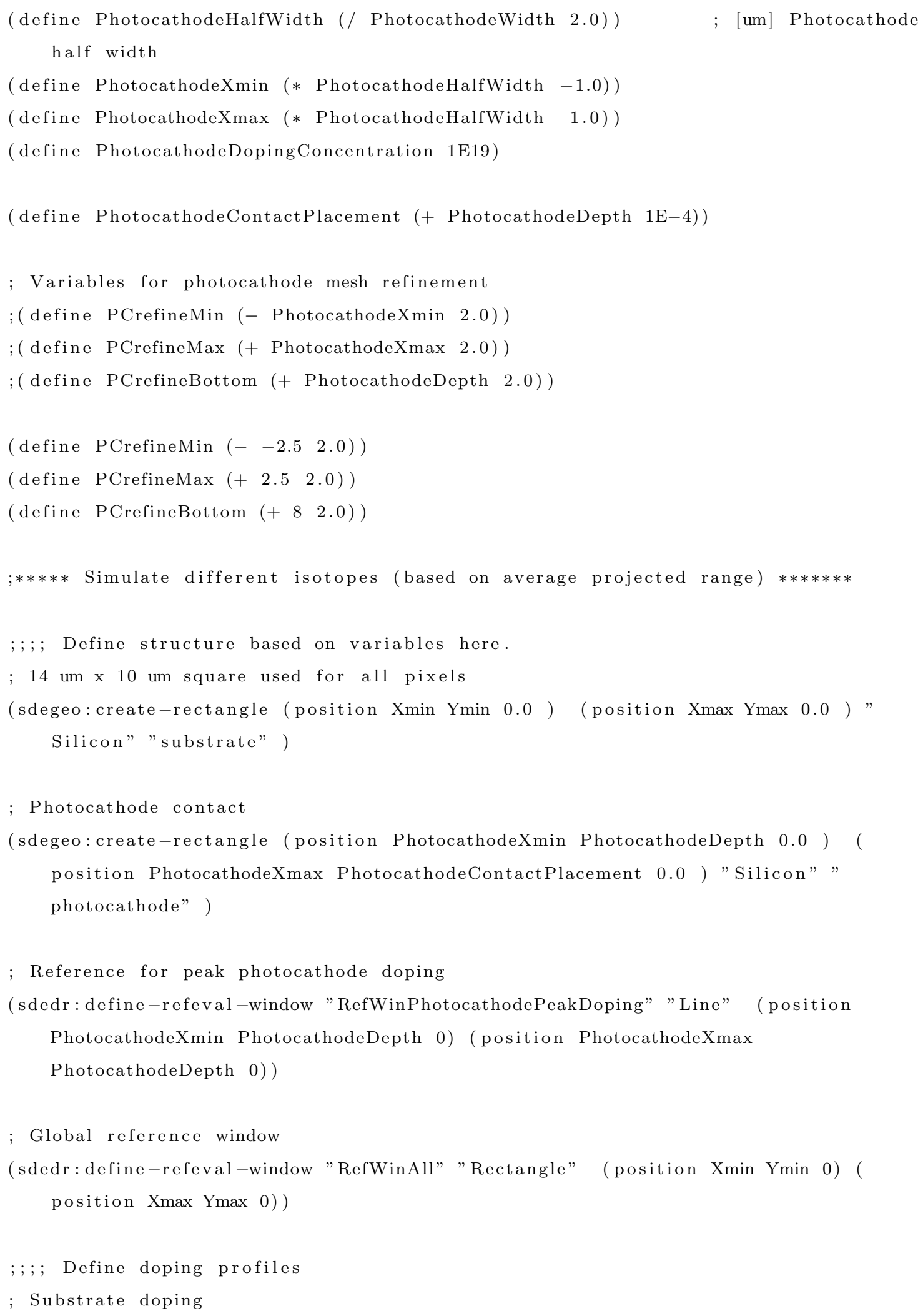




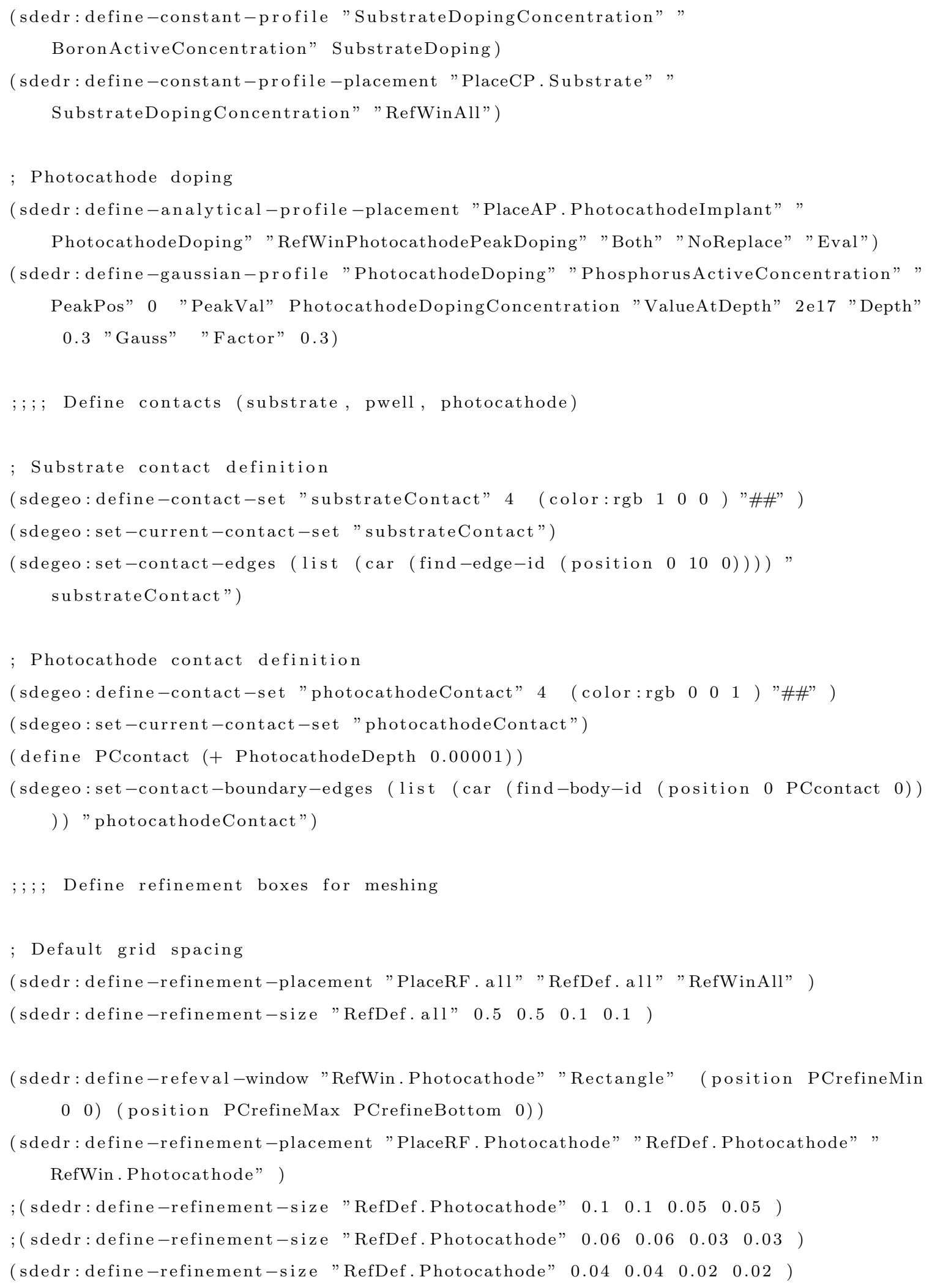




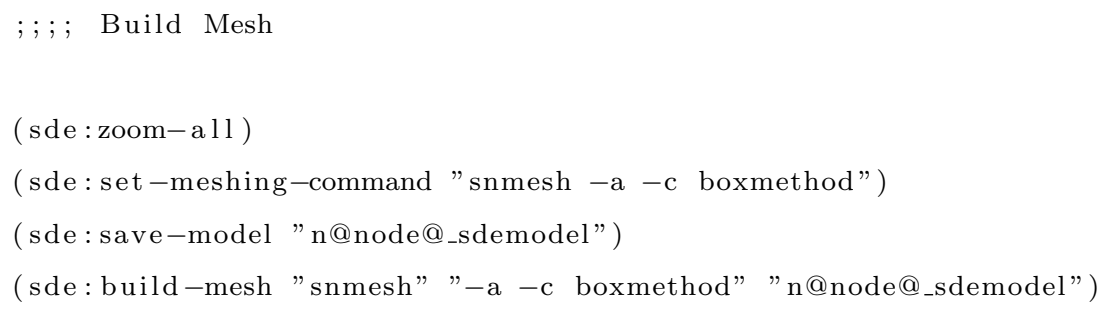

\section{A.2 2D Substrate Diode Device Simulation}

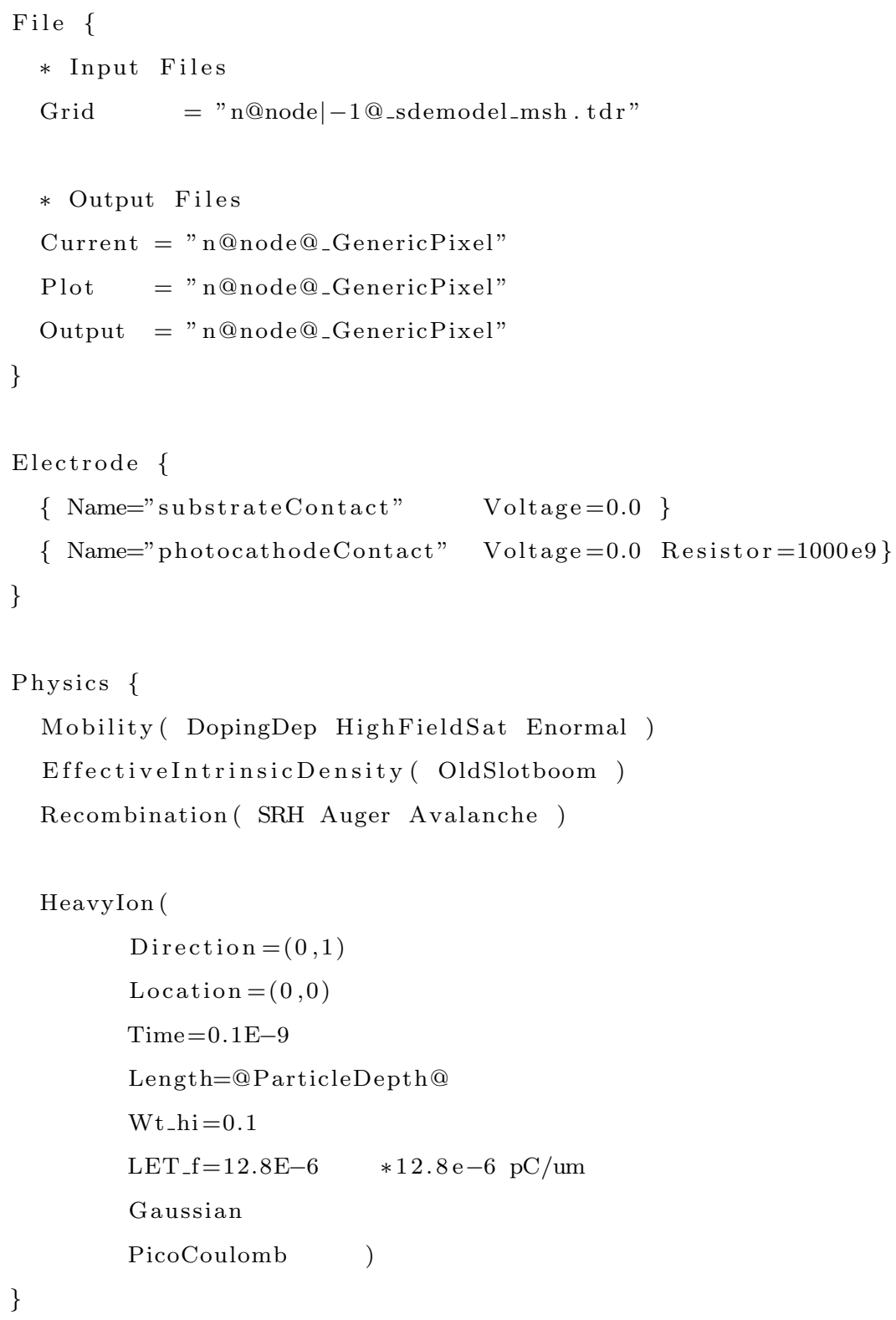




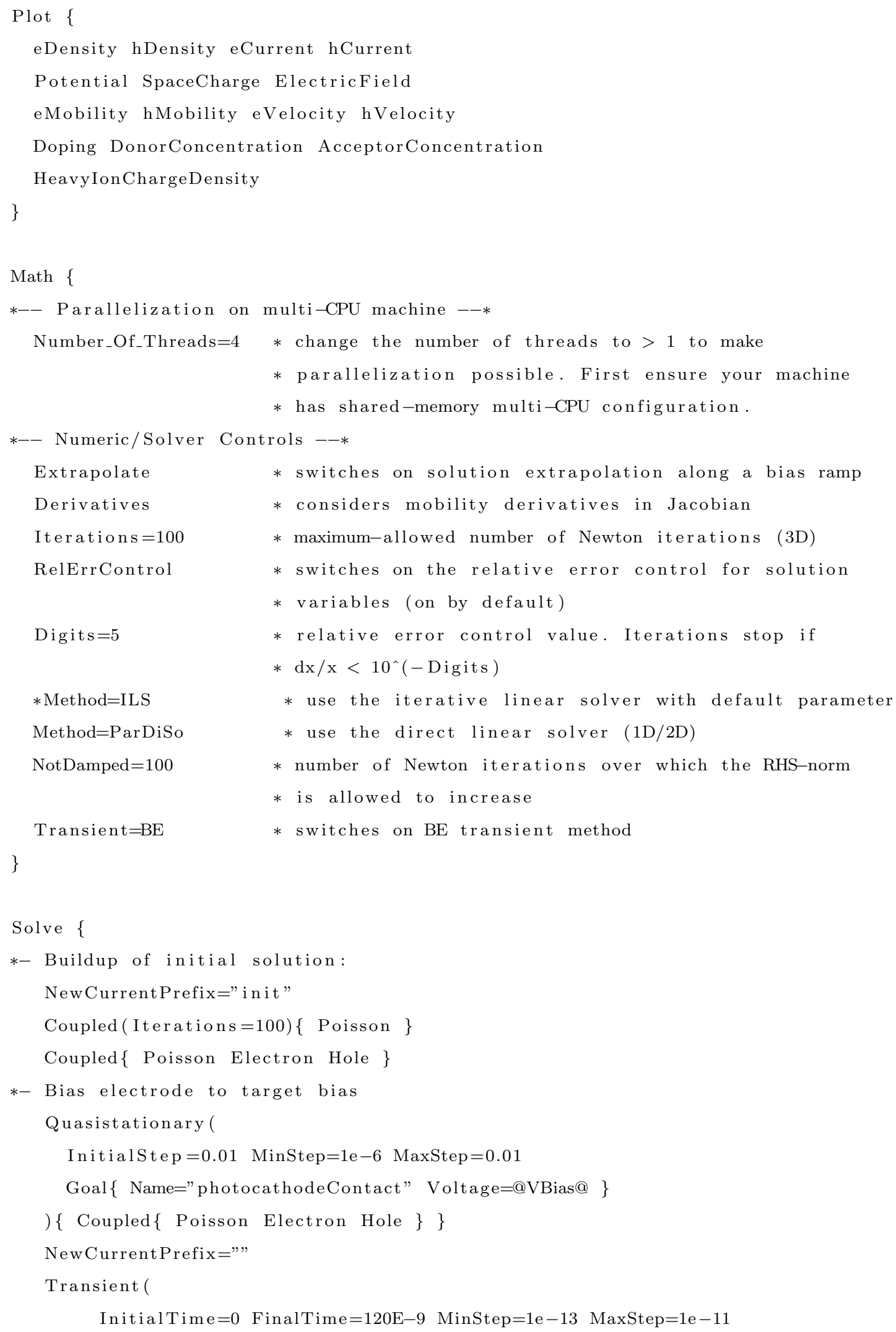


) $\{$ Coupled $\{$ Poisson Electron Hole $\}$ \}

\}

\section{A.3 2D 705S Structure}

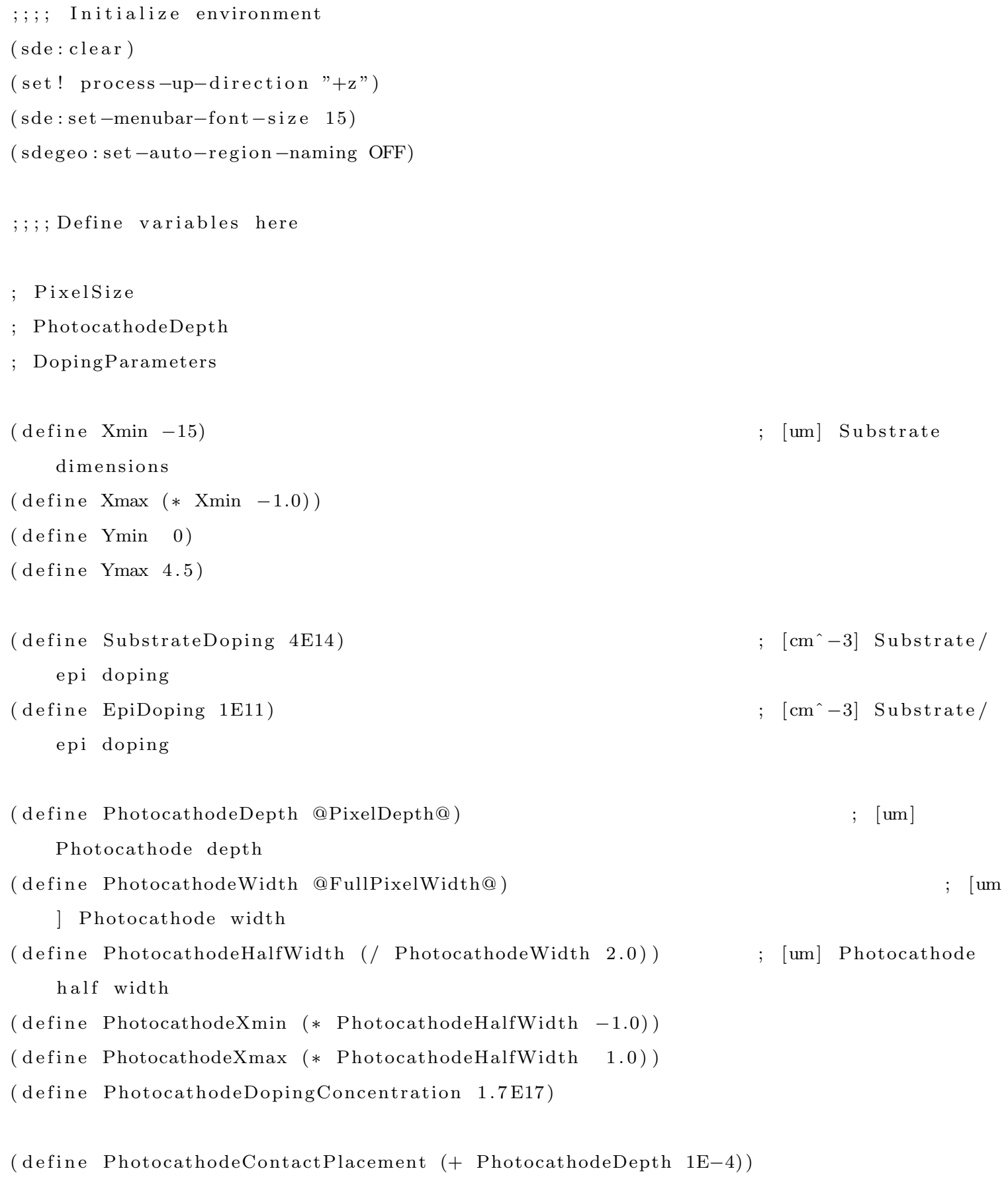




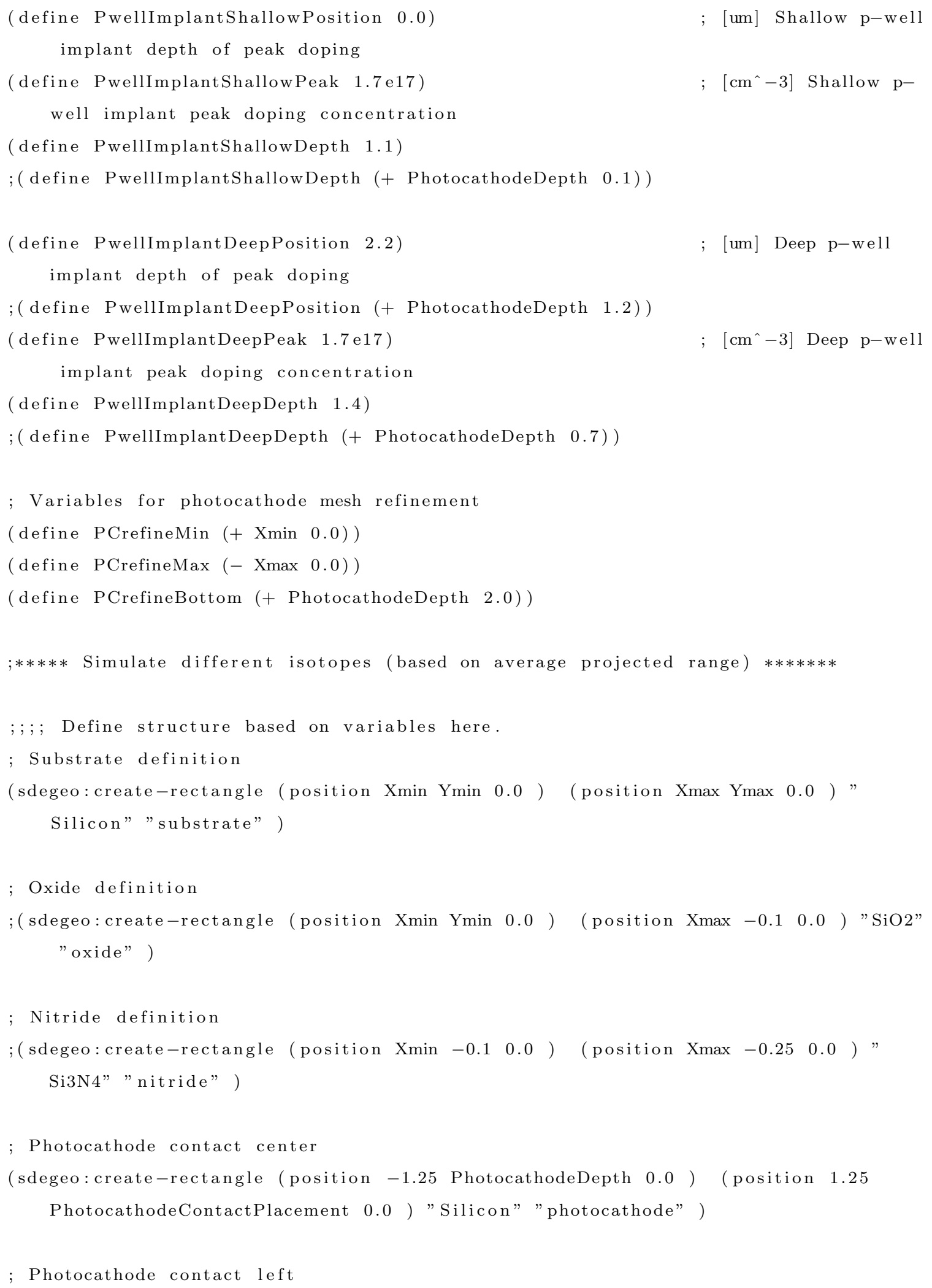




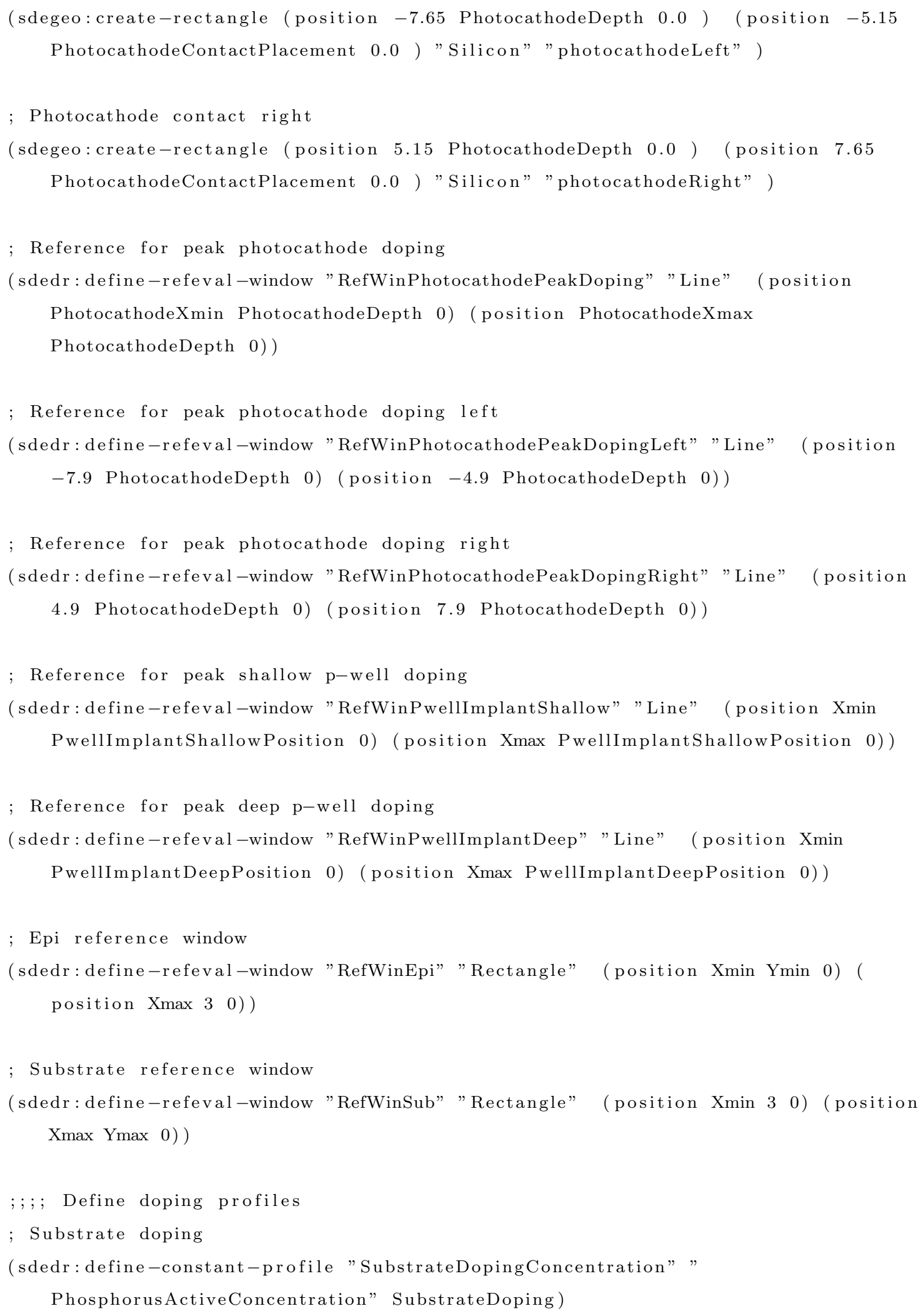




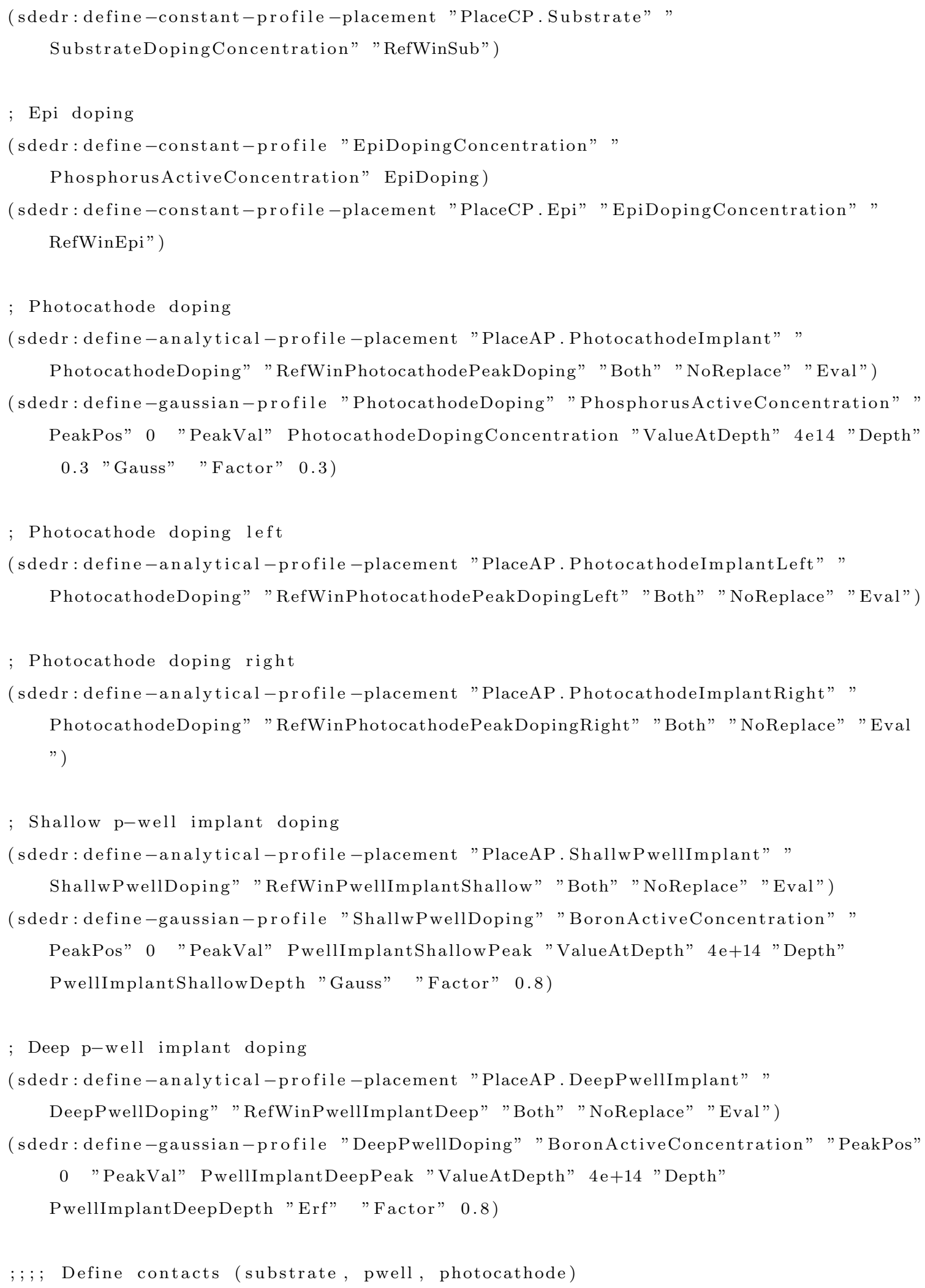




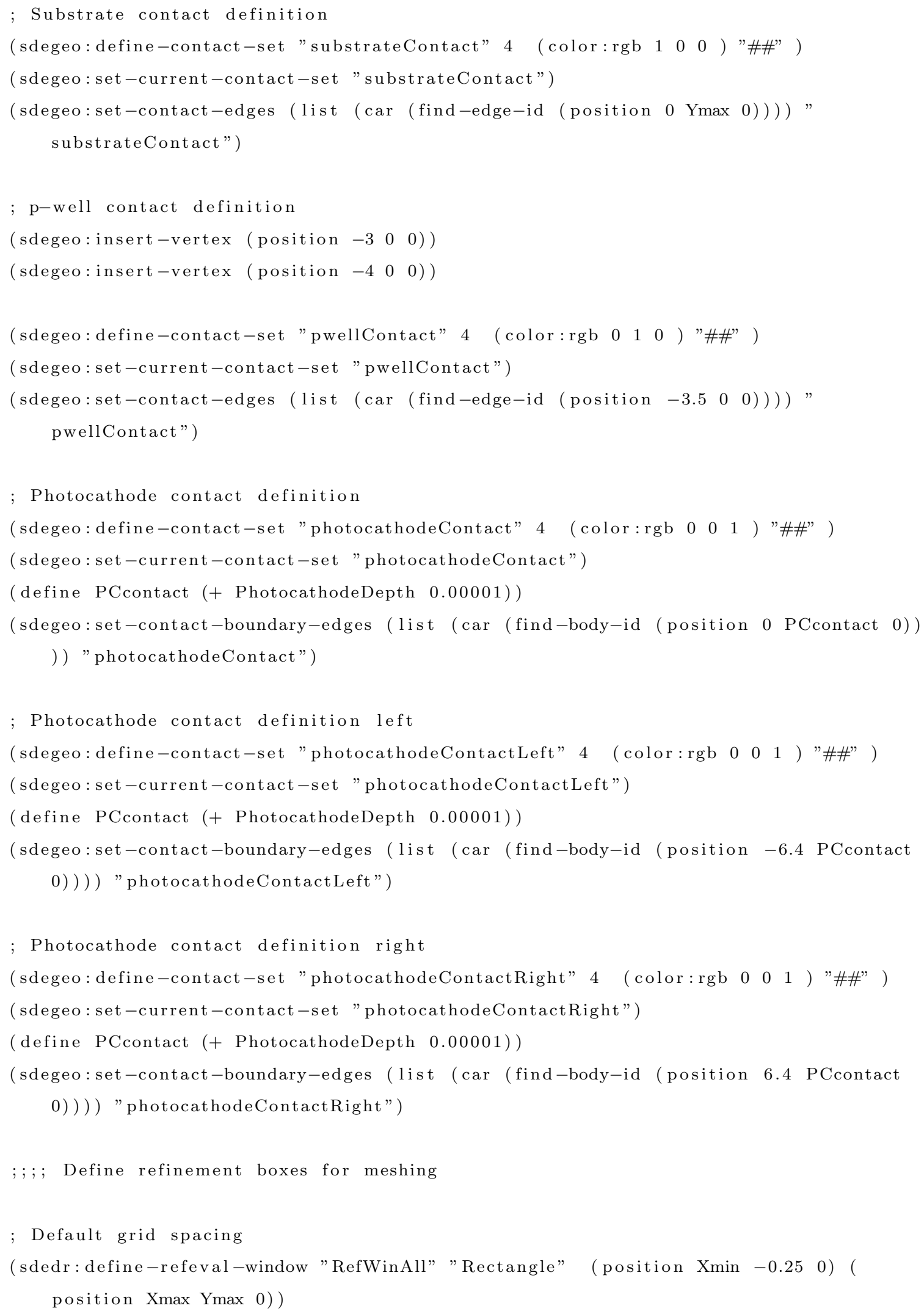




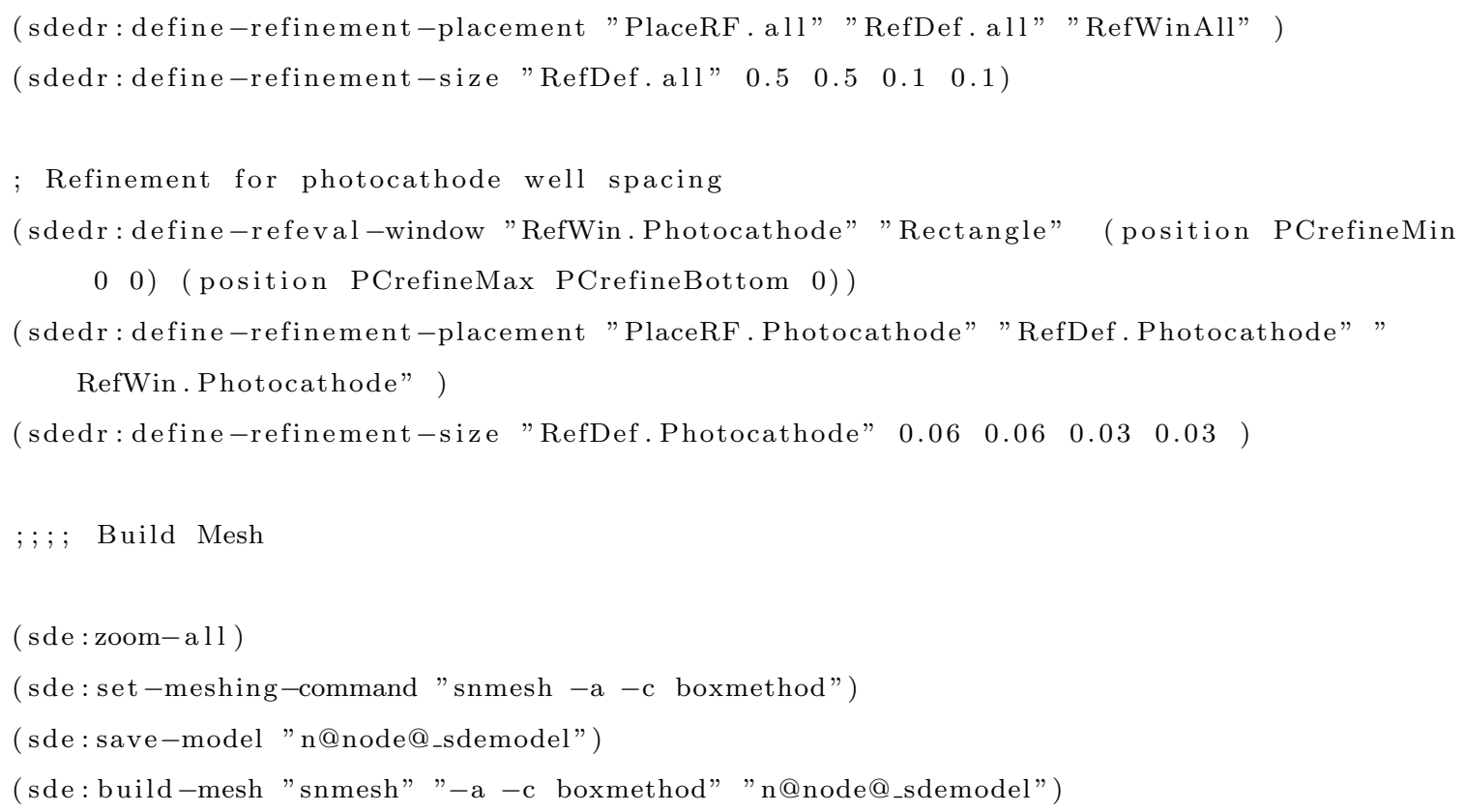

\section{A.4 2D 705S Device Simulation}

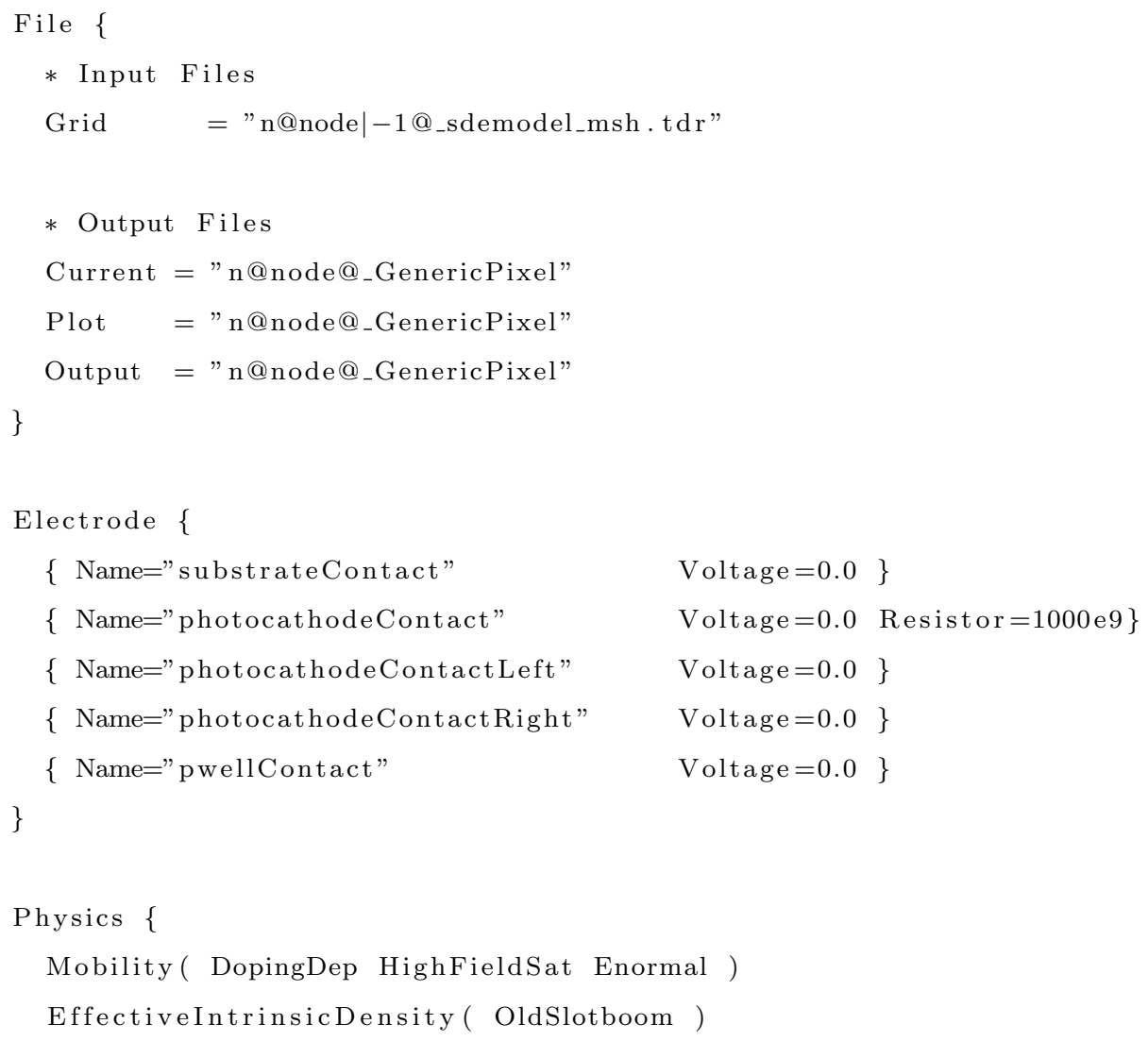




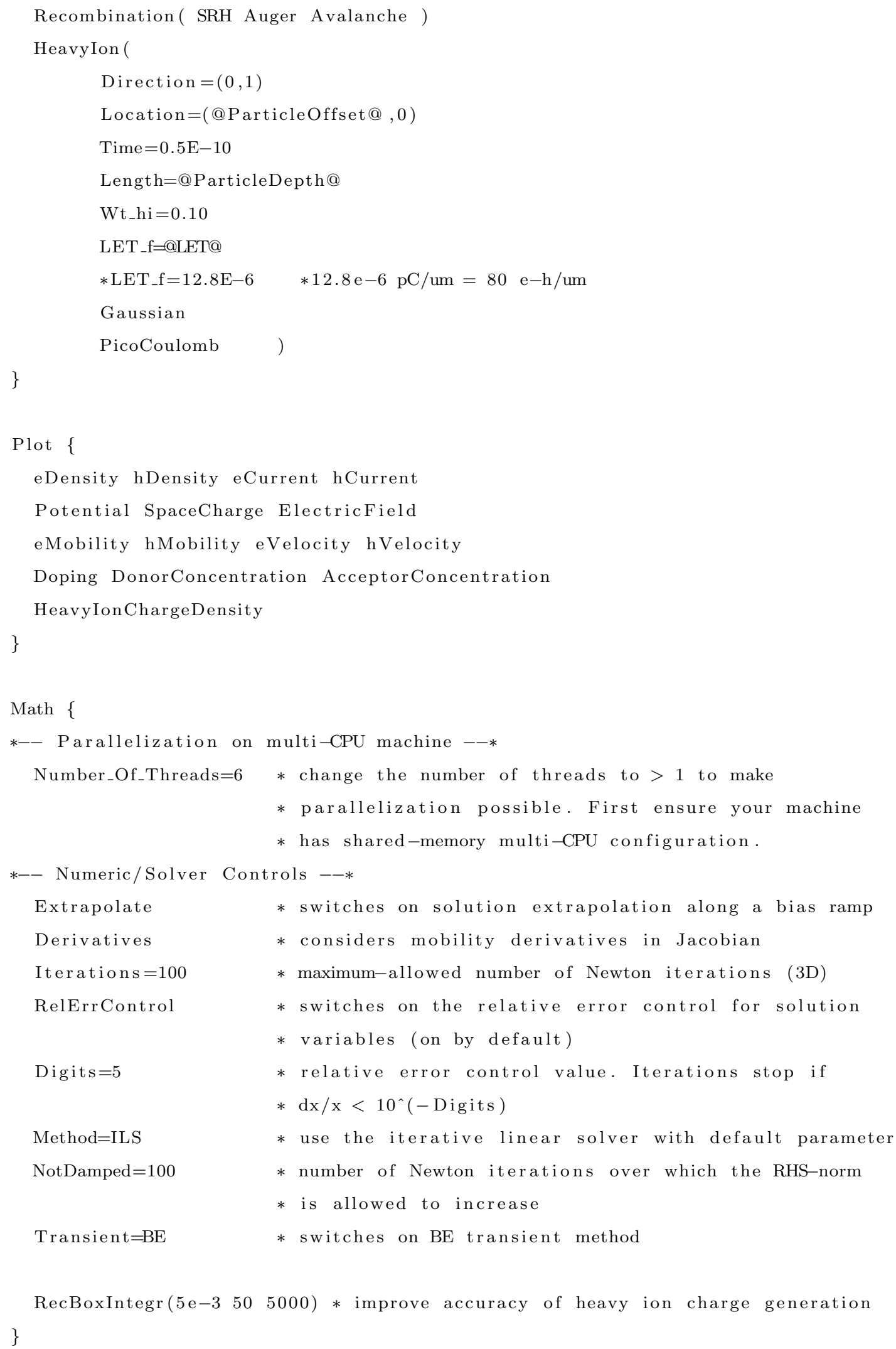




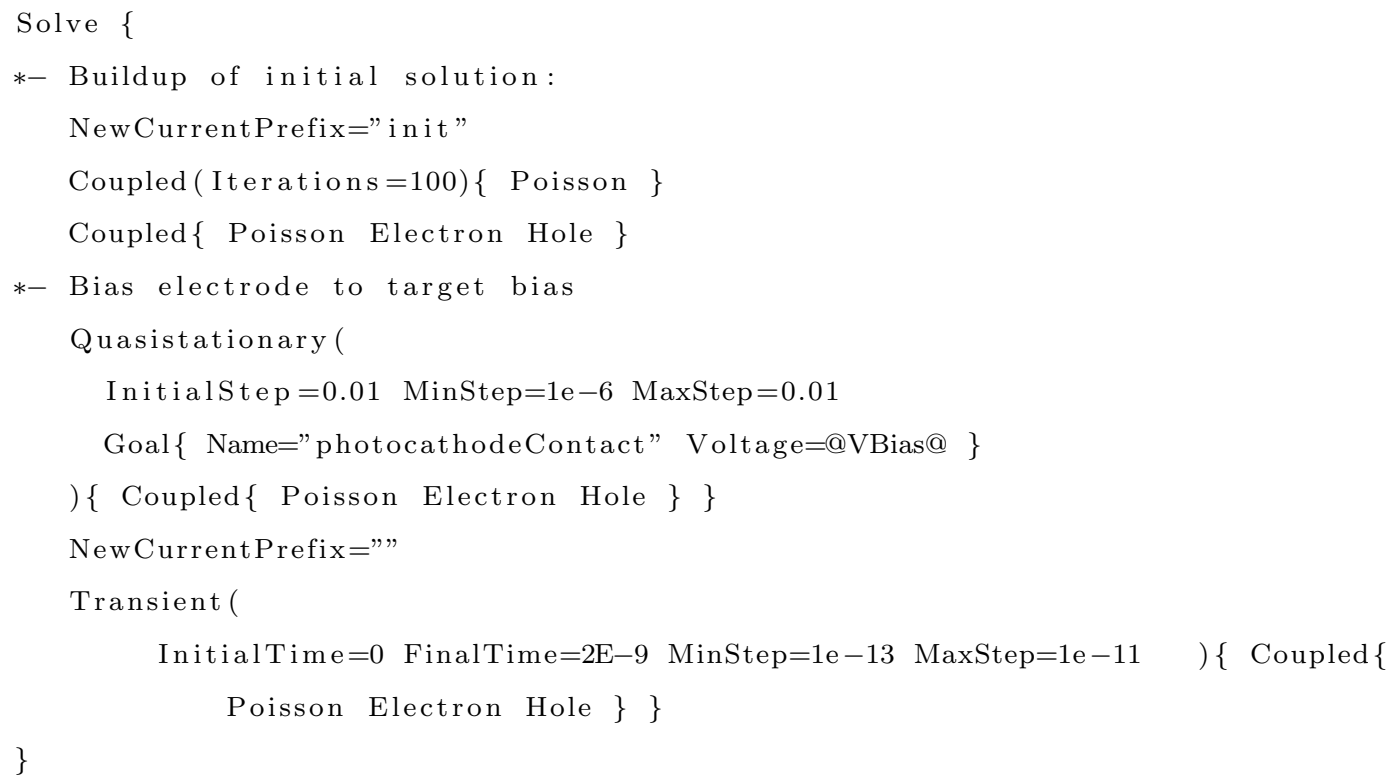

\section{A.5 3D 705S Structure}

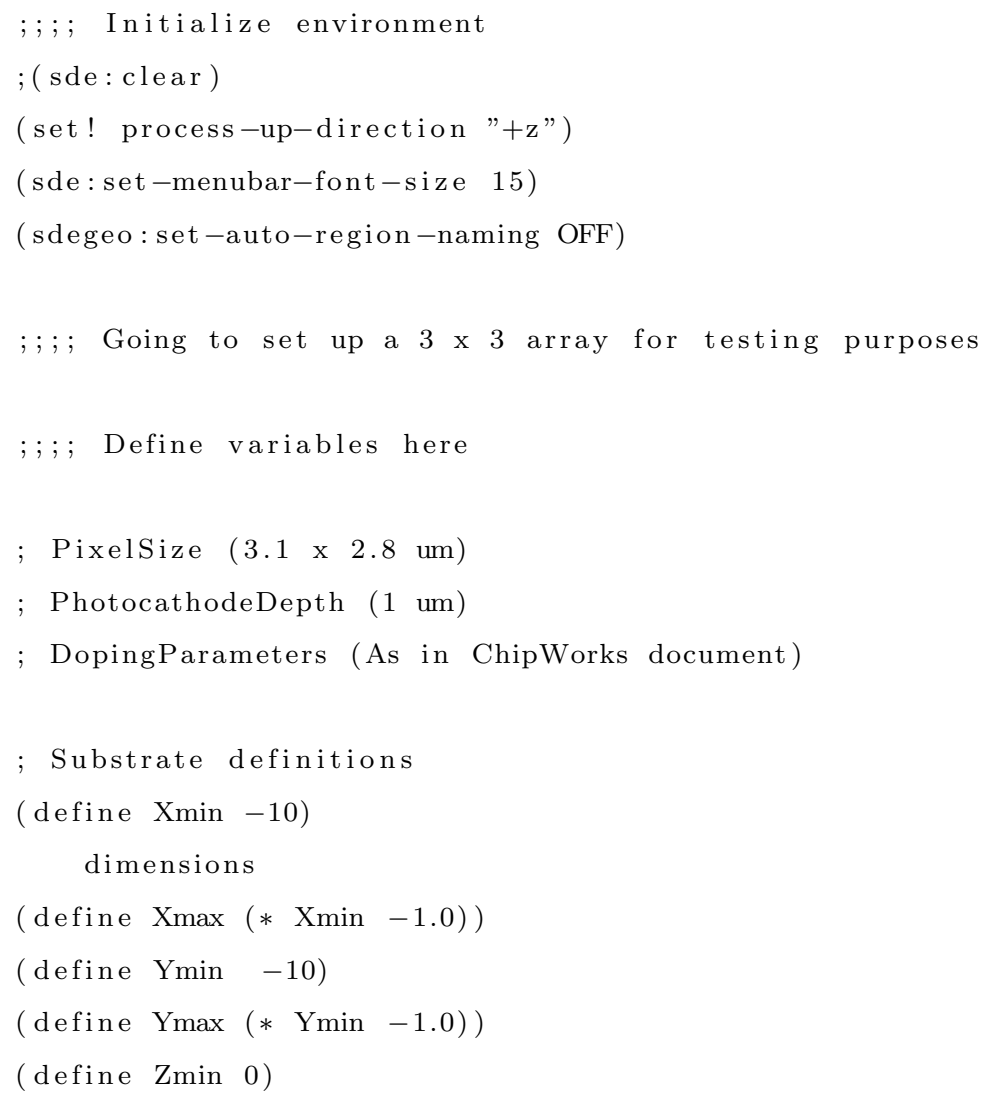




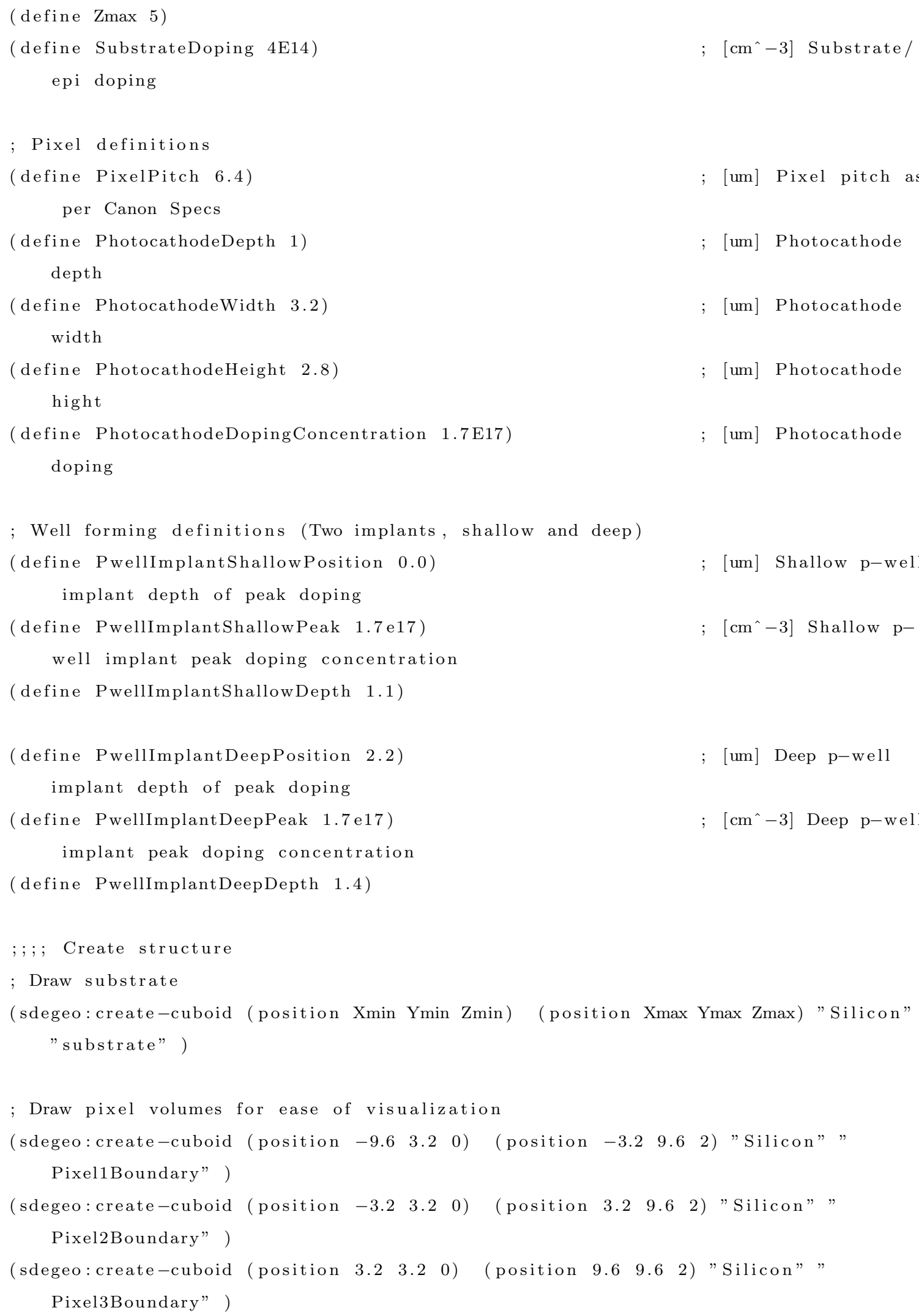




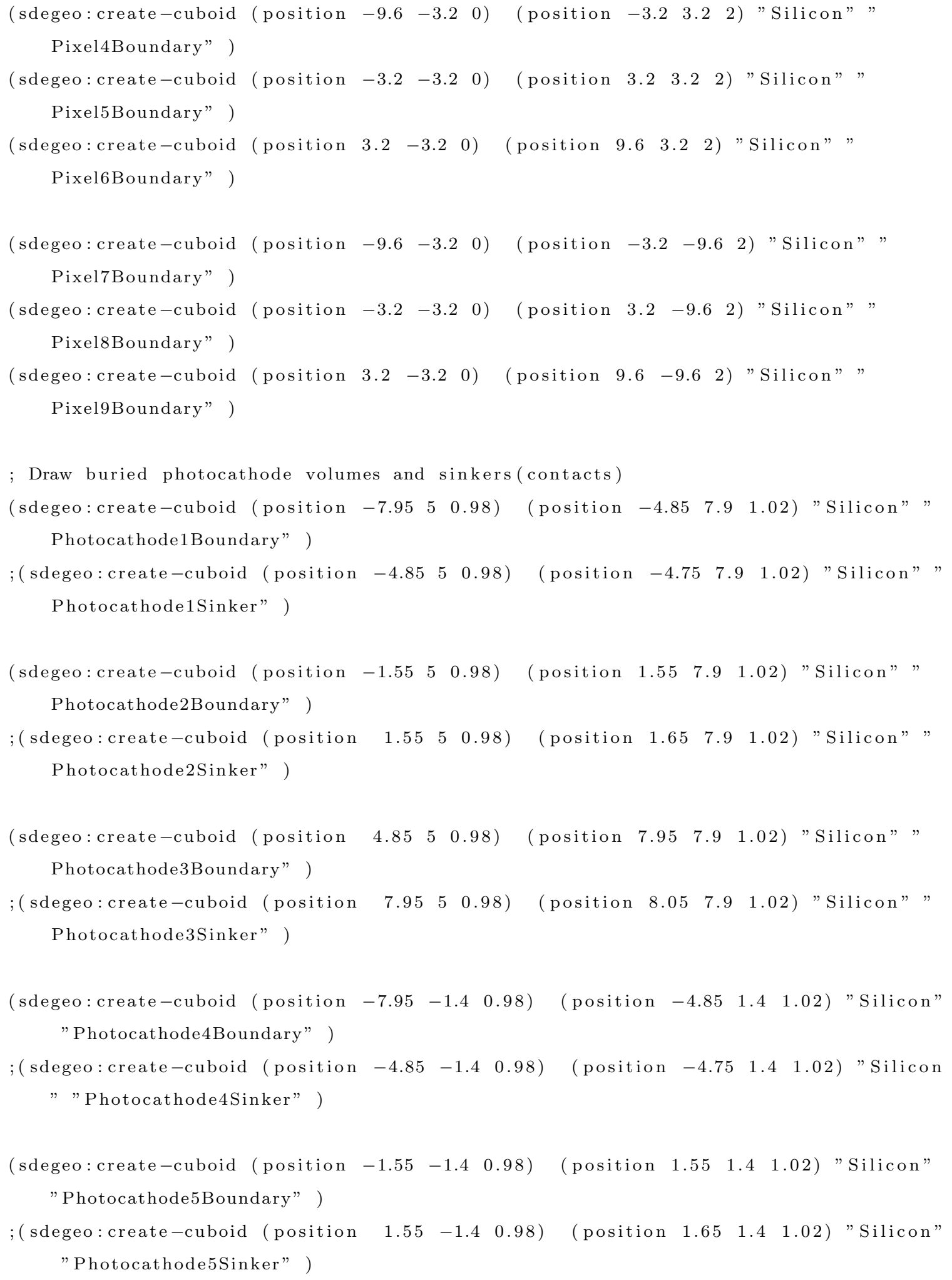




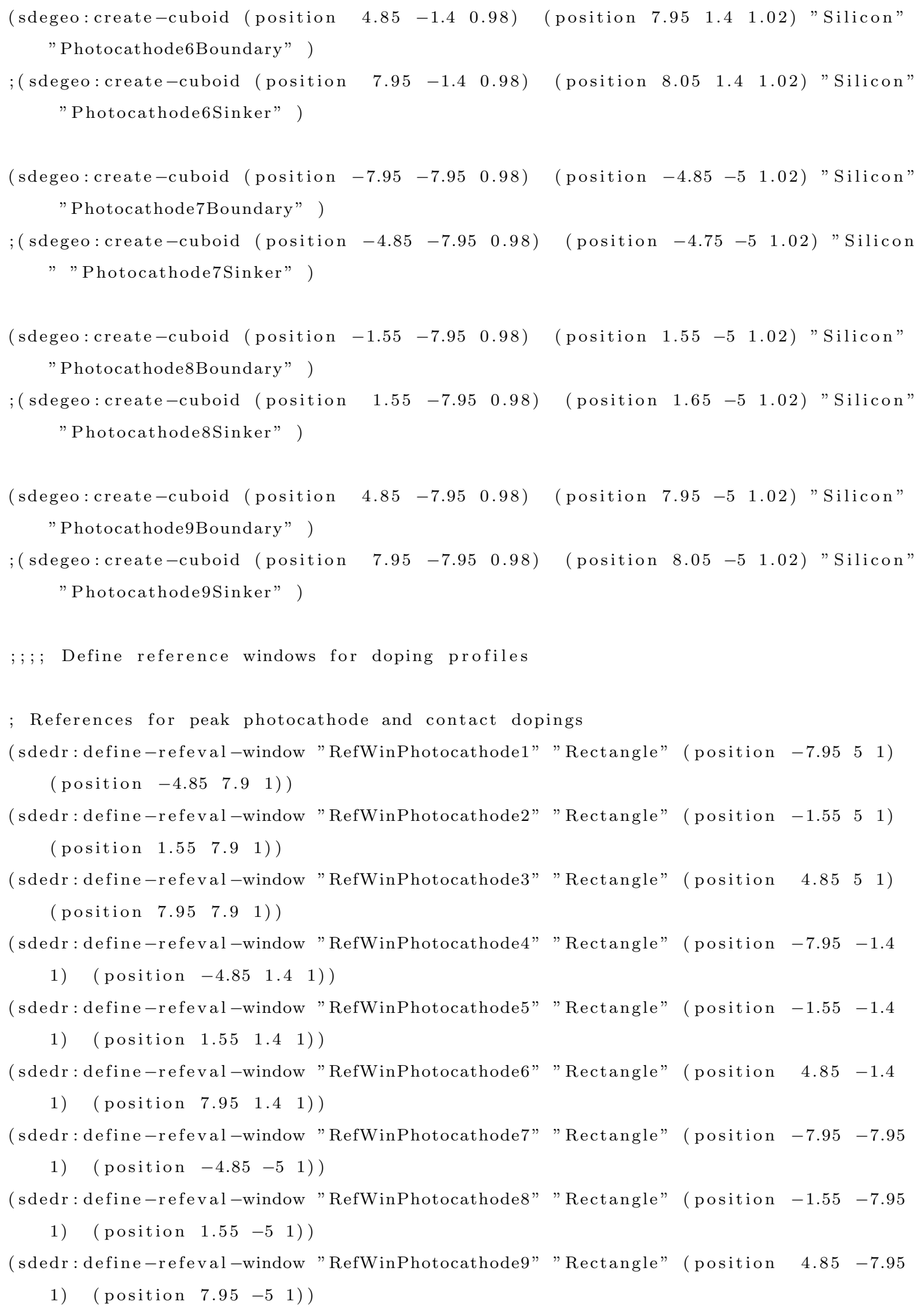




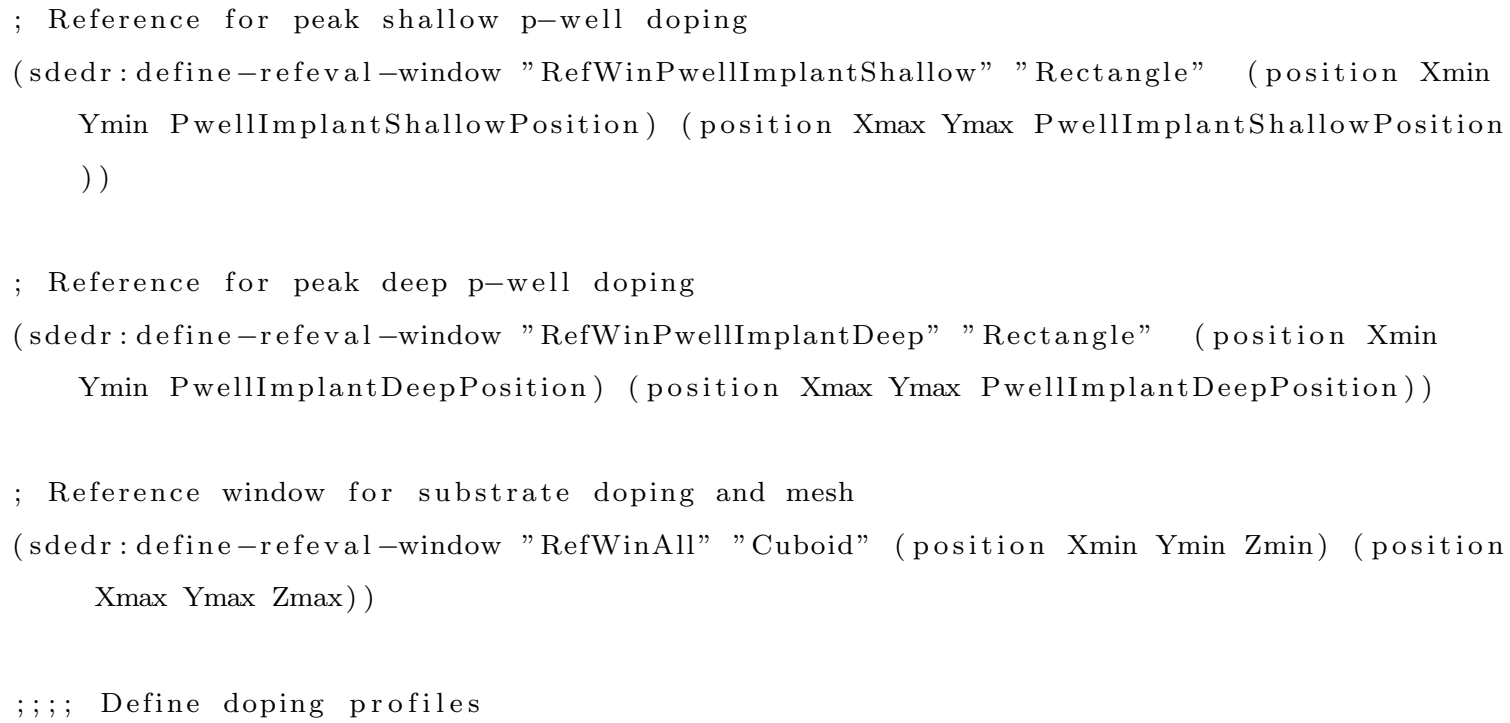




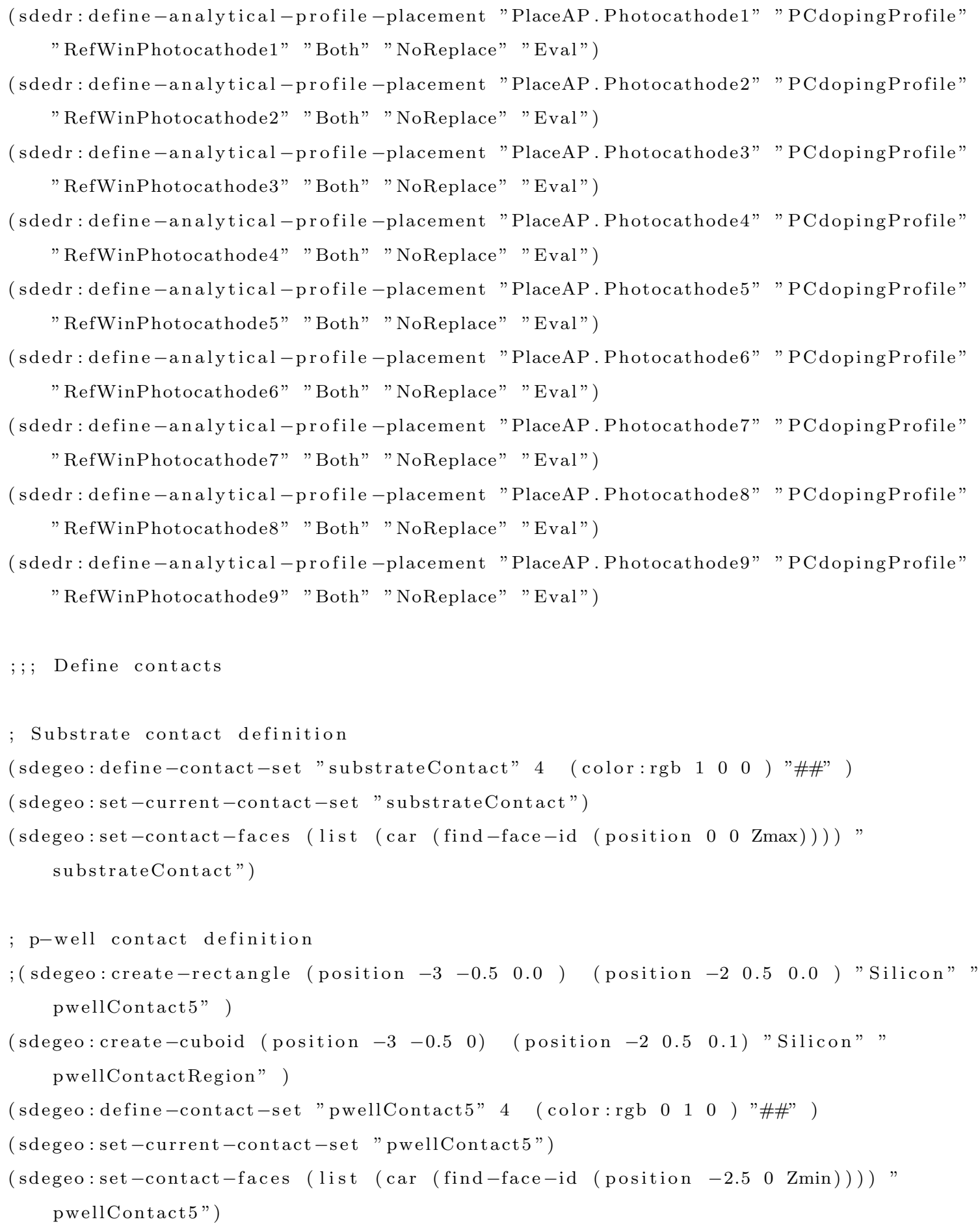




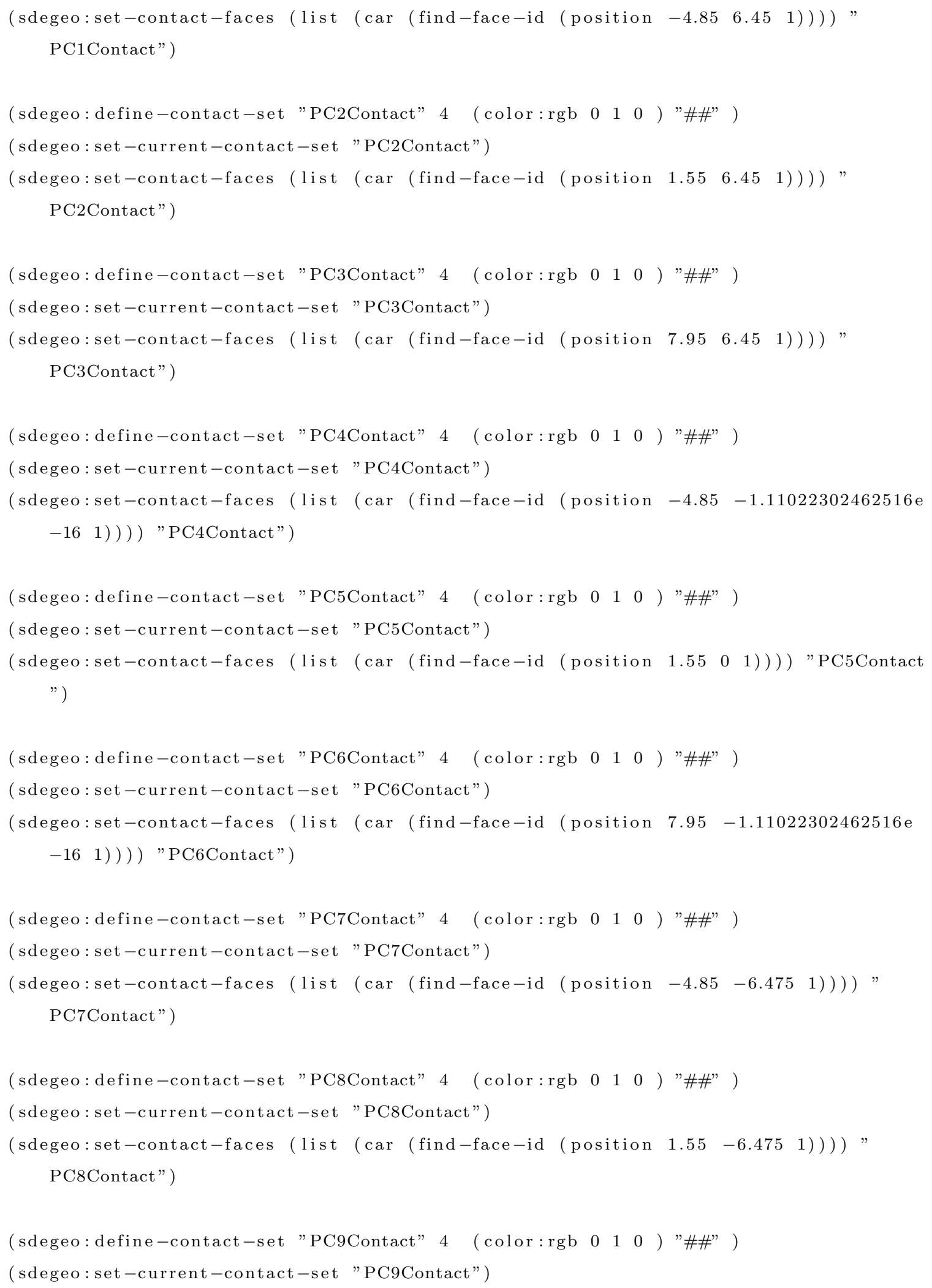




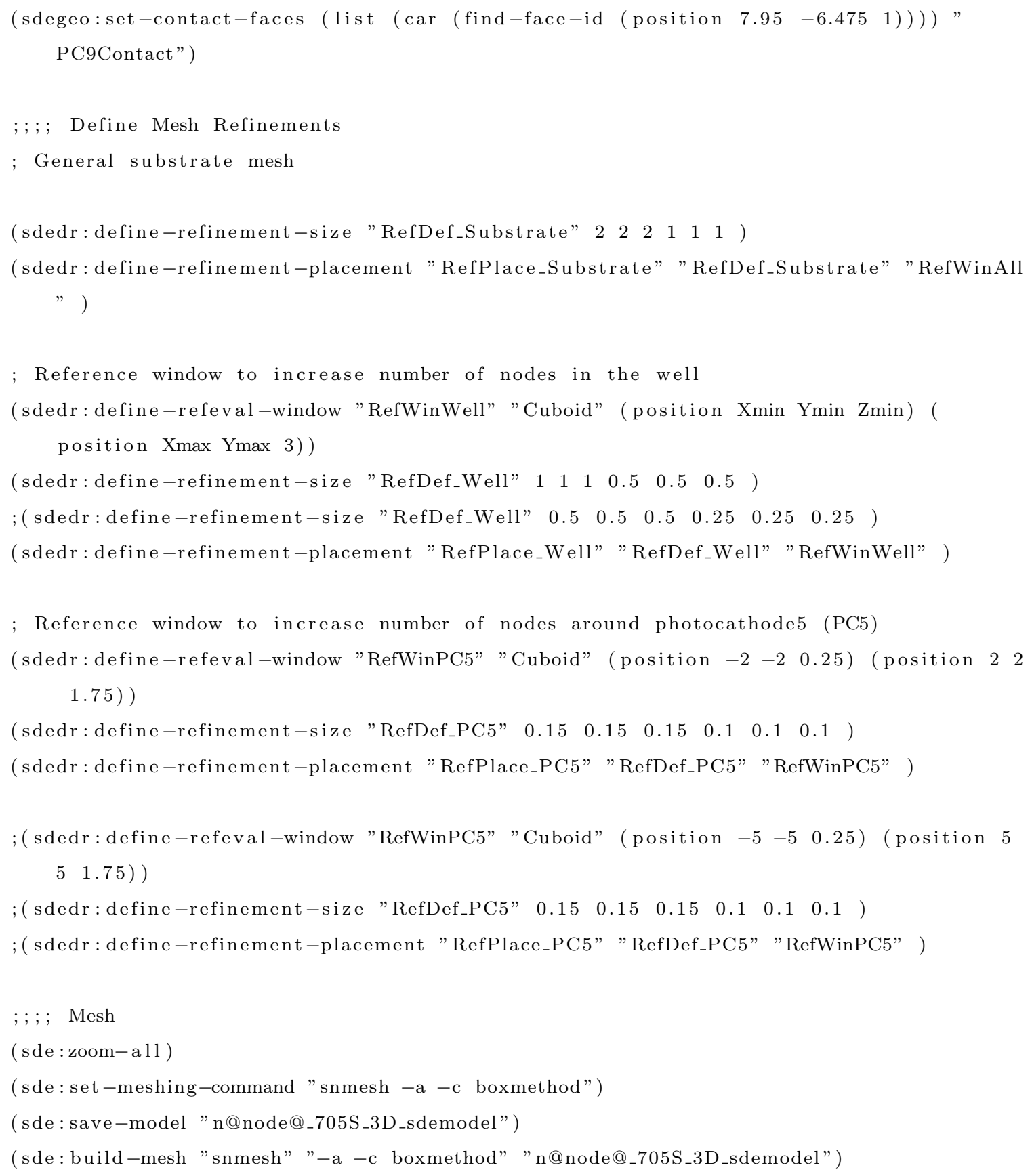

\section{A.6 3D 705S Device Simulation}

File \{

* Input Files

Grid = "n@node $\mid-1 @$ @705S_3D_sdemodel_msh.tdr" 


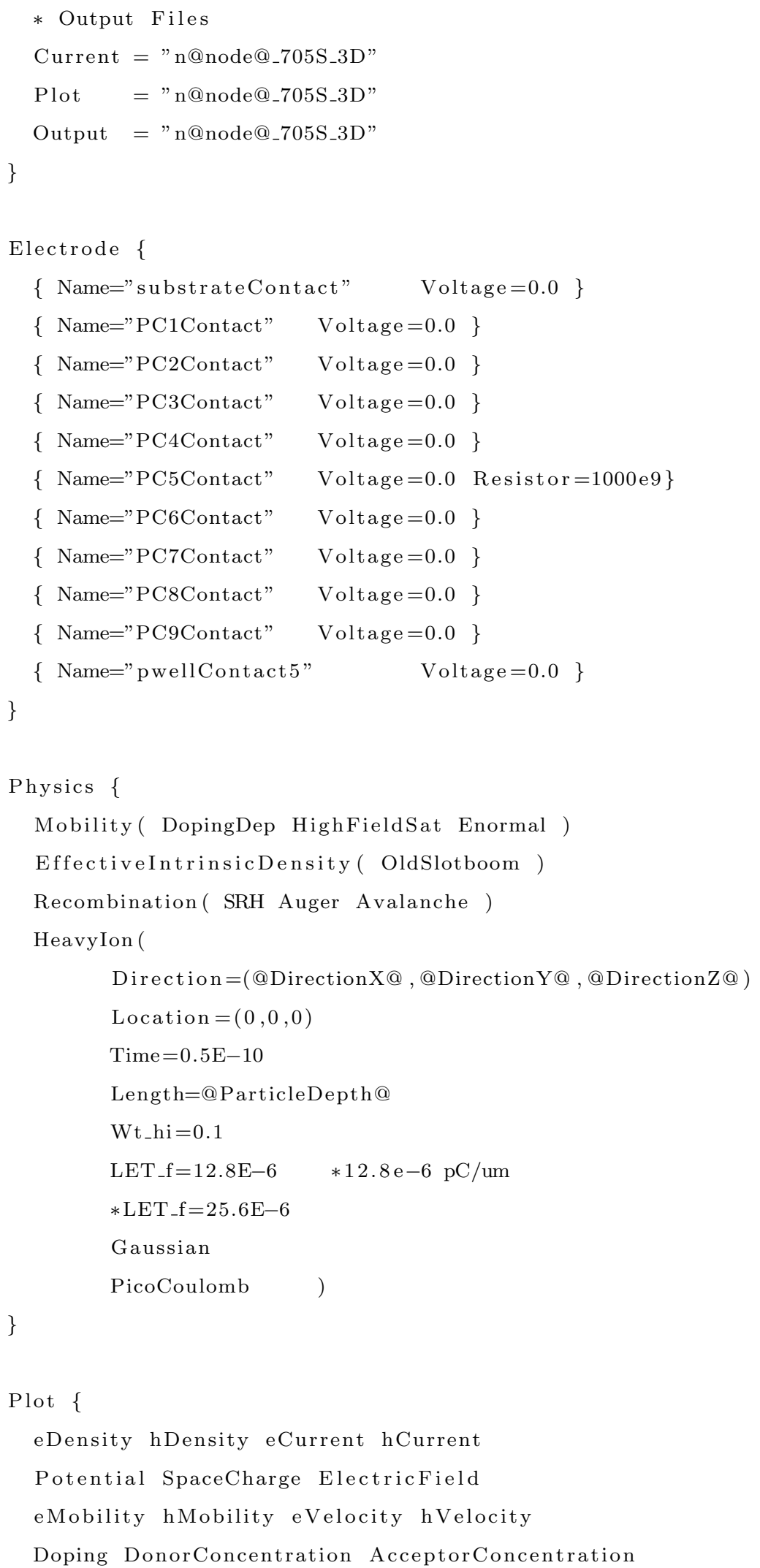




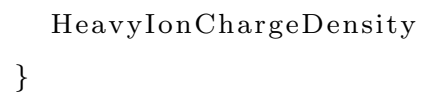




\section{Appendix B}

\section{Raw Chelation Data}

B.1 Data from Figure 5.4(c) 


\begin{tabular}{|c|c|c|c|c|c|c|c|c|c|c|c|c|c|c|c|c|c|}
\hline & $\stackrel{0}{0}$ & $\begin{array}{l}\mathscr{\infty} \\
\infty \\
\infty\end{array}$ & 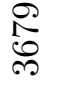 & $\begin{array}{l}0 \\
10 \\
\infty \\
\infty\end{array}$ & $\underset{\infty}{\stackrel{10}{二}}$ & $\vec{\sim}$ & $\overparen{8}$ & 0 & $\stackrel{+}{+\infty}$ & $\begin{array}{l}0 \\
\infty \\
10\end{array}$ & $\stackrel{8}{8}$ & $\stackrel{8}{\infty}$ & $\stackrel{\mathscr{\rho}}{\infty}$ & $\underset{\substack{\infty \\
+\infty}}{\infty}$ & $\overrightarrow{\mathscr{8}}$ & 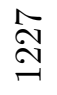 & 0 \\
\hline & $\stackrel{10}{\longrightarrow}$ & 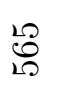 & $\sqrt[6]{6}$ & $\begin{array}{l}1 \\
0 \\
\infty\end{array}$ & $\sqrt{6}$ & 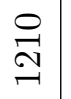 & $\stackrel{\infty}{\infty}$ & 0 & $\begin{array}{l}\stackrel{0}{ } \\
\stackrel{D}{0}\end{array}$ & $\begin{array}{l}\mathscr{8} \\
\stackrel{\leftrightarrow}{0}\end{array}$ & $\begin{array}{c}0 \\
0 \\
0\end{array}$ & 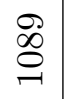 & $\mathscr{8}$ & $\overrightarrow{\mathscr{\sigma}}$ & 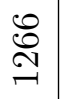 & 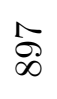 & 0 \\
\hline & $\underset{工}{ت}$ & 翮 & 簓 & $\begin{array}{l}0 \\
\mathbb{N} \\
\stackrel{\Theta}{-}\end{array}$ & 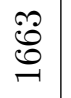 & $\stackrel{\infty}{\underset{N}{N}}$ & $\stackrel{\stackrel{\overbrace{}}{ᄋ}}{\circ}$ & 0 & $\overrightarrow{\stackrel{F}{S}}$ & $\begin{array}{l}12 \\
\stackrel{2}{8} \\
\stackrel{2}{S}\end{array}$ & $\frac{\sqrt{-}}{6}$ & 今. & $\mid \begin{array}{l}2 \mathscr{0} \\
\stackrel{\mathscr{N}}{N}\end{array}$ & $\stackrel{10}{\stackrel{10}{H}}$ & $\stackrel{ }{\circ}$ & $\underset{-}{\stackrel{O}{\circ}}$ & 0 \\
\hline & $\stackrel{m}{-1}$ & $\stackrel{?}{R}$ & $\begin{array}{l}\sqrt{20} \\
\stackrel{2}{9} \\
\stackrel{9}{-1}\end{array}$ & $\underset{20}{71}$ & $\stackrel{20}{\mathscr{g}}$ & $\overrightarrow{\mathscr{9}}$ & $\begin{array}{l}\stackrel{\infty}{\infty} \\
\stackrel{0}{\circ}\end{array}$ & 0 & $\underset{\overparen{J}}{\stackrel{N}{0}}$ & $\begin{array}{l}\stackrel{Q}{\mathscr{C}} \\
\stackrel{N}{N}\end{array}$ & $\stackrel{\mathcal{\Xi}}{\exists}$ & $\underset{0}{-1}$ & 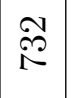 & 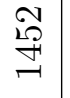 & $\stackrel{\sim}{\stackrel{\sim}{*}}$ & $\begin{array}{l}\infty \\
\infty \\
\stackrel{\infty}{10} \\
-1\end{array}$ & 0 \\
\hline & $\stackrel{\sim}{\mathcal{I}}$ & 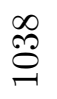 & 융 & 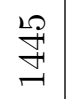 & $\begin{array}{l}10 \\
8 \\
8\end{array}$ & 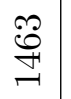 & $\begin{array}{l}18 \\
\infty \\
\infty\end{array}$ & 0 & 药 & $\ddot{\infty}$ & 点 & $\begin{array}{l}0 \\
0 \\
0\end{array}$ & $\left|\begin{array}{c}\infty \\
\infty \\
\infty\end{array}\right|$ & $\stackrel{m}{\stackrel{m}{N}}$ & 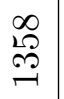 & $\stackrel{10}{\stackrel{2}{\Xi}}$ & 0 \\
\hline & $\exists$ & $\stackrel{\mathscr{N}}{\Xi}$ & $\vec{\sigma}$ & 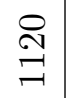 & $\begin{array}{l}20 \\
\mathscr{\sigma}\end{array}$ & $\stackrel{\sqrt[50]{9}}{+1}$ & $\ddot{\sigma}$ & 0 & 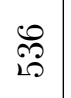 & 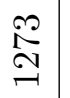 & $\stackrel{8}{8}$ & กี & $\stackrel{\mathfrak{P}}{\tilde{i}}$ & $\stackrel{0}{ \pm}$ & $\underset{H}{\stackrel{H}{J}}$ & 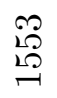 & 0 \\
\hline & $\stackrel{ }{-}$ & $\stackrel{\infty}{\underset{J}{J}}$ & $\begin{array}{l}\infty \\
\stackrel{2}{\circ}\end{array}$ & $\begin{array}{c}N \\
\infty \\
\infty\end{array}$ & $\stackrel{0}{0}$ & 望 & 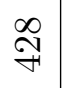 & 0 & $\stackrel{\mathbb{N}}{\underset{H}{*}}$ & नै & $\begin{array}{l}\infty \\
\stackrel{1}{1} \\
1\end{array}$ & ชิ & $\mid \begin{array}{l}10 \\
\infty\end{array}$ & 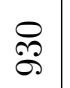 & 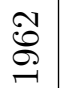 & $\begin{array}{l}\infty \\
0 \\
0 \\
\end{array}$ & 0 \\
\hline & $a$ & $\stackrel{10}{\overparen{H}}$ & $\underset{\sigma}{\sigma}$ & $\stackrel{+}{R}$ & $\stackrel{12}{80}$ & 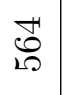 & 爷 & 0 & 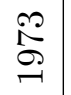 & 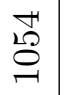 & 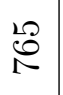 & $\stackrel{\mathscr{D}}{\mathscr{0}}$ & $\underset{\mathbb{N}}{\mathfrak{N}}$ & \begin{tabular}{l}
\multirow{2}{*}{} \\
$\infty$ \\
-1
\end{tabular} & 占 & $\begin{array}{l}\vec{\infty} \\
\infty \\
-1\end{array}$ & 0 \\
\hline & $\infty$ & $\vec{F}$ & $\stackrel{\mathscr{P}}{\underset{F}{二}}$ & $\begin{array}{l}\infty \\
\infty \\
\infty\end{array} \mid$ & $\stackrel{\vec{m}}{=}$ & 8 & $\underset{F}{\stackrel{ }{F}}$ & 0 & 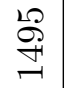 & $\underset{\Xi}{\vec{b}}$ & $\begin{array}{l}0 \\
\stackrel{2}{2} \\
\stackrel{+}{+}\end{array}$ & $\begin{array}{c}\infty \\
\stackrel{9}{\sharp} \\
\underset{f}{f}\end{array}$ & $\frac{12}{6}$ & $\underset{\stackrel{ }{+}}{ }$ & $\underset{\sim}{\stackrel{J}{\rightleftarrows}}$ & $\underset{\sim}{\stackrel{\infty}{N}}$ & 0 \\
\hline & $N$ & $\stackrel{\stackrel{20}{\sim}}{\stackrel{\sim}{\sim}}$ & $\stackrel{\Re}{\infty}$ & \begin{tabular}{c}
$\mathscr{g}$ \\
\multirow{+}{\pm}{} \\
-1
\end{tabular} & $\begin{array}{l}+1 \\
6 \\
0 \\
0\end{array}$ & $\mathscr{\mathscr { g }}$ & 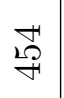 & 0 & $\begin{array}{l}\stackrel{20}{O} \\
\stackrel{-}{-1}\end{array}$ & $\begin{array}{l}0 \\
2 \\
\infty \\
\infty \\
-1\end{array}$ & $\begin{array}{l}\stackrel{2}{\infty} \\
\stackrel{\infty}{+}\end{array}$ & $\stackrel{9}{+1}$ & $\vec{J}$ & $\stackrel{8}{2}$ & $\begin{array}{l}\sqrt{20} \\
20 \\
\end{array}$ & $\begin{array}{l}10 \\
0 \\
0 \\
0\end{array}$ & 0 \\
\hline & 0 & 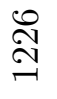 & 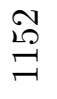 & $\begin{array}{l}\vec{I} \\
\stackrel{-}{二}\end{array}$ & 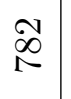 & $\begin{array}{l}0 \\
18 \\
0\end{array}$ & $\begin{array}{l}\text { 袩 } \\
\end{array}$ & 0 & $\stackrel{20}{9}$ & $\begin{array}{l}\infty \\
\underset{+}{\infty}\end{array}$ & 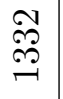 & $\stackrel{\sim}{\stackrel{F}{\leftrightarrows}}$ & $\ddot{\mathscr{\infty}}$ & 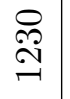 & $\begin{array}{l}\infty \\
0 \\
0 \\
\stackrel{9}{\rightarrow}\end{array}$ & 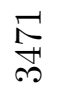 & 0 \\
\hline & 10 & $\begin{array}{l}0 \\
\stackrel{2}{1} \\
\stackrel{9}{*}\end{array}$ & $\underset{\exists}{\stackrel{F}{Z}}$ & $\begin{array}{l}\tilde{S} \\
\stackrel{\rho}{\oplus}\end{array}$ & $\begin{array}{l}\stackrel{1}{2} \\
\stackrel{2}{n}\end{array}$ & $\stackrel{m}{i}$ & $\vec{B}$ & 0 & $\stackrel{8}{8}$ & బิ & $\underset{\sim}{\stackrel{N}{\sim}}$ & 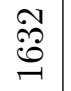 & 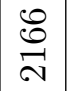 & $\begin{array}{l}\infty \\
2 \\
\stackrel{2}{N}\end{array}$ & 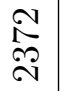 & 감 & 0 \\
\hline & + & $\begin{array}{l}\stackrel{\circ}{\circ} \\
\stackrel{\leftrightarrow}{N}\end{array}$ & $\stackrel{\stackrel{N}{\Im}}{\stackrel{\sim}{\sim}}$ & \begin{tabular}{l}
$\infty$ \\
$\infty$ \\
\multirow{N}{N}{}
\end{tabular} \mid & $\begin{array}{l}12 \\
\infty \\
= \\
=\end{array}$ & $\begin{array}{l}\stackrel{0}{\mathscr{D}} \\
\stackrel{N}{\sim}\end{array}$ & 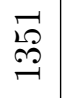 & 0 & 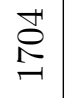 & $\underset{\infty}{\stackrel{\leftrightarrow}{O}}$ & $\underset{\sim}{\stackrel{\overbrace{}}{二}}$ & $\begin{array}{l}\stackrel{\leftrightarrow}{\circ} \\
\stackrel{9}{-}\end{array}$ & $\underset{\sim}{\stackrel{F}{H}}$ & $\begin{array}{l}\stackrel{N}{\infty} \\
\stackrel{N}{N}\end{array}$ & $\underset{\stackrel{\sim}{\stackrel{N}{N}}}{ }$ & $\underset{\sim}{\stackrel{N}{N}}$ & 0 \\
\hline & $\infty$ & 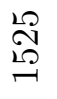 & 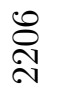 & $\underset{\mathscr{O}}{\stackrel{\Xi}{O}}$ & 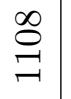 & $\begin{array}{l}\stackrel{2}{2} \\
\stackrel{9}{-}\end{array}$ & $\begin{array}{l}\stackrel{\mathscr{g}}{\stackrel{\rightrightarrows}{二}} \\
\end{array}$ & 0 & 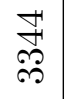 & $\underset{\sim}{\stackrel{F}{*}}$ & $\stackrel{8}{\varnothing}$ & 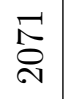 & 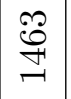 & $\begin{array}{l}\stackrel{\sim}{\infty} \\
= \\
=\end{array}$ & $\begin{array}{l}\infty \\
\infty \\
\infty \\
\infty \\
-1\end{array}$ & 前 & 0 \\
\hline & $N$ & $\begin{array}{l}\sqrt{20} \\
\stackrel{20}{2}\end{array}$ & $\begin{array}{l}R \\
\stackrel{R}{F}\end{array}$ & $\begin{array}{l}\infty \\
\infty \\
20 \\
-1\end{array} \mid$ & $\begin{array}{l}10 \\
5\end{array}$ & 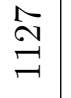 & $\begin{array}{l}\stackrel{N}{2} \\
\stackrel{2}{N}\end{array}$ & 0 & $\stackrel{\overrightarrow{9}}{=}$ & $\stackrel{\stackrel{9}{9}}{\rightarrow}$ & 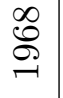 & $\stackrel{\sqrt{-1}}{20}$ & $\begin{array}{l}0 \\
0 \\
0\end{array}$ & 茴 & $\stackrel{m}{\sigma}$ & 岕 & 0 \\
\hline & - & 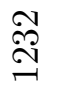 & $\begin{array}{l}\stackrel{N}{\stackrel{N}{N}} \\
\stackrel{N}{N}\end{array}$ & हे & 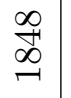 & $\begin{array}{l}\stackrel{\ominus}{A} \\
\stackrel{\sim}{N}\end{array}$ & $\begin{array}{l}\stackrel{\sim}{\sigma} \\
\stackrel{\sigma}{\sigma}\end{array}$ & 0 & $\begin{array}{l}20 \\
0 \\
0 \\
0\end{array}$ & $\begin{array}{l}\stackrel{゚}{\stackrel{N}{\sim}} \\
\stackrel{\sim}{\sim}\end{array}$ & $\stackrel{\mathscr{O}}{\mathscr{N}}$ & $\begin{array}{l}\text { กิ } \\
\text { ̊ิ) }\end{array}$ & 总 & 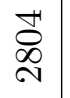 & $\underset{⿱}{\vec{m}}$ & $\overbrace{0}^{0}$ & \\
\hline & & -1 & N & $\infty$ & $\nabla$ & 20 & 0 & $\sim$ & $\infty$ & $\sigma$ & $\stackrel{ }{\circ}$ & $\exists$ & $\stackrel{\mathcal{N}}{\sim}$ & $\stackrel{m}{\sim}$ & 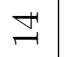 & $\stackrel{10}{\rightarrow}$ & $\stackrel{0}{-1}$ \\
\hline & & & & & & & & & & & & & & & & & \\
\hline
\end{tabular}

